\title{
Pd-Catalyzed Dearomative Three-Component Reaction of Bromoarenes with Diazo Compounds and Allylborates
}

\author{
Masaaki Komatsuda, Hiroki Kato, Kei Muto, ${ }^{*}$ and Junichiro Yamaguchi* \\ Department of Applied Chemistry, Waseda University, 3-4-1 Ohkubo, Shinjuku, Tokyo 169-8555, Japan \\ E-mail: keimuto@aoni.waseda.jp, junyamaguchi@waseda.jp
}

\section{Table of Contents}

1. General

2. Synthesis of Bromoarenes

3. Pd-Catalyzed Dearomative Three-Component Reaction of Bromoarenes

4. One-Pot Dearomative Reaction of 2-Phenylthiophene (6)

5. Application to A Pharmaceutical Compound

6. Derivatization of Products

7. Effect of Parameters

8. References

9. ${ }^{1} \mathrm{H}$ and ${ }^{13} \mathrm{C}$ NMR Spectra

10. Crude ${ }^{1} \mathrm{H}$ NMR Spectra of 4 and 8
$\mathrm{S} 2$

S3-S9

S10-S20

S20-S21

S21-S22

S22-S26

S27-S29

S30

S31-S102

S103-S127 


\section{General}

Unless otherwise noted, all reactants or reagents including dry solvents were obtained from commercial suppliers and used as received. (Trimethylsilyl)diazomethane solution 2.0 M in diethyl ether was obtained from Sigma-Aldrich. 4-(Dimethylamino)phenyldiphenylphosphine (L1), 1-bromonaphthalene (1C), 5-bromoacenaphthene (1G), 4-bromocumene (1I), (4-bromophenyl)methanol (10), 1-bromo-3,5-dimethylbenzene (1P), 1-bromo-3,5-dimethoxybenzene (1Q), 4-bromo-3-fluorotoluene (1R), 2-bromo-5-phenylthiophene (1S), and ticlopidine hydrochloride were obtained from TCI Chemical. Potassium allyltrifluoroborate was obtained from Frontier Scientific. Potassium fluoride and 4-bromotoluene $(\mathbf{1 H})$ were obtained from KANTO Chemical. 2-(4-Bromophenyl)-1,3-dioxolane $\quad(\mathbf{1 N}),{ }^{[1]} \quad$ 2-(5-bromothiophen-2-yl)pyridine $\quad(\mathbf{1 U}),{ }^{[2]} \quad$ and 2-bromo-5-methylthiophene (1V) ${ }^{[3]}$ were synthesized according to procedures and the spectra matched with those of compounds reported in the literature. Unless otherwise noted, all reactions were performed with dry solvents under an atmosphere of $\mathrm{N}_{2}$ in dried glassware using standard vacuum-line techniques. All dearomative three-component reactions were performed in $25-\mathrm{mL}$ glass vessel tubes equipped with a screw cap and heated (IKA Plate RCT digital) in a 16-well aluminum reaction block (IKA DB5.4 Block) unless otherwise noted. All work-up and purification procedures were carried out with reagent-grade solvents in air.

Analytical thin-layer chromatography (TLC) was performed using Silica-gel 70 TLC Plate-Wako $(0.25 \mathrm{~mm})$. The developed chromatogram was analyzed by UV lamp $(254 \mathrm{~nm})$. Flash column chromatography was performed with Biotage Isolera ${ }^{\circledR}$ equipped with Biotage SNAP Cartridge KP-Sil columns and hexane/EtOAc as an eluent unless otherwise noted. Preparative thin-layer chromatography (PTLC) was performed using Wakogel B5-F silica coated plates $(0.75 \mathrm{~mm})$ prepared in our laboratory. Basic alumina chromatography was performed using basic alumina, activated $(\mathrm{pH}=$ 9.0-11.0) from Wako Pure Chemical. High-resolution mass spectra were conducted on Thermo Fisher Scientific ExactivePlus (ESI and DART). Nuclear magnetic resonance (NMR) spectra were recorded on a JEOL JNM-ECS-400 ( $\left.{ }^{1} \mathrm{H} 400 \mathrm{MHz},{ }^{13} \mathrm{C} 101 \mathrm{MHz}\right)$ spectrometer. Chemical shifts for ${ }^{1} \mathrm{H}$ NMR are expressed in parts per million (ppm) relative to tetramethylsilane $(\delta 0.00 \mathrm{ppm})$. Chemical shifts for ${ }^{13} \mathrm{C}$ NMR are expressed in ppm relative to $\mathrm{CDCl}_{3}(\delta 77.0 \mathrm{ppm})$. Data are reported as follows: chemical shift, multiplicity $(\mathrm{s}=$ singlet, $\mathrm{d}=$ doublet, $\mathrm{dd}=$ doublet of doublets, $\mathrm{ddd}=$ doublet of doublets of doublets, $\mathrm{t}=$ triplet, $\mathrm{dt}=$ doublet of triplets, $\mathrm{td}=$ triplet of doublets, $\mathrm{q}=$ quartet, $\mathrm{m}=$ multiplet, $\mathrm{brs}=$ broad singlet), coupling constant $(\mathrm{Hz})$, and integration. 


\section{Synthesis of Bromoarenes}

\section{Synthesis of $1 \mathrm{~A}$ and $1 B$}<smiles>[R]c1cccc2ccccc12</smiles><smiles>CN(C)Cc1ccccc1</smiles><smiles>[R]c1ccc(Br)c2ccccc12</smiles>

To a solution of naphthalenes (1.0 equiv) in $\mathrm{MeCN}(0.30 \mathrm{M})$ was added $N$-bromosuccinimide (NBS: 1.2 equiv). After stirring the mixture for several hours with monitoring reaction progress by TLC, the solvent was evaporated in vacuo. Purification by flash column chromatography afforded the corresponding bromoarene.<smiles>Cc1ccc(Br)c2ccccc12</smiles>

\section{1-Bromo-4-methylnaphthalene $(1 \mathrm{~A})^{[4]}$}

Purification by flash column chromatography (hexane) afforded 1A (968 mg, $5.0 \mathrm{mmol} \mathrm{scale}$, $87 \%$ yield) as a colorless oil. The spectra are in accordance with those of the compounds reported in the literature.<smiles>CCc1ccc(Br)c2ccccc12</smiles>

\section{1-Bromo-4-ethylnaphthalene (1B)}

Purification by flash column chromatography (hexane) afforded 1B (705 mg, $3.0 \mathrm{mmol} \mathrm{scale,}$ quant.) as a colorless oil. ${ }^{1} \mathrm{H} \mathrm{NMR}\left(400 \mathrm{MHz}, \mathrm{CDCl}_{3}\right) \delta 8.25(\mathrm{~d}, J=8.0 \mathrm{~Hz}, 1 \mathrm{H}), 7.97(\mathrm{~d}, J=8.0 \mathrm{~Hz}$, $1 \mathrm{H}), 7.64(\mathrm{~d}, J=8.0 \mathrm{~Hz}, 1 \mathrm{H}), 7.52(\mathrm{dd}, J=8.0,6.8 \mathrm{~Hz}, 1 \mathrm{H}), 7.49(\mathrm{dd}, J=8.0,6.8 \mathrm{~Hz}, 1 \mathrm{H}), 7.10(\mathrm{~d}, J$ $=8.0 \mathrm{~Hz}, 1 \mathrm{H}), 2.99(\mathrm{q}, J=7.6 \mathrm{~Hz}, 2 \mathrm{H}), 1.30(\mathrm{t}, J=7.6 \mathrm{~Hz}, 3 \mathrm{H}) ;{ }^{13} \mathrm{C} \mathrm{NMR}\left(101 \mathrm{MHz}, \mathrm{CDCl}_{3}\right) \delta 140.3$, 133.0, 131.9, 129.6, 127.8, 126.7, 126.4, 125.3, 124.1, 120.7, 25.7, 14.8; HRMS (DART) $\mathrm{m} / \mathrm{z}$ calcd for $\mathrm{C}_{12} \mathrm{H}_{11} \mathrm{Br}[\mathrm{M}]^{+}: 234.0039$ found 234.0033.

\section{Synthesis of 1D and 1E}<smiles>OCCc1cccc2ccccc12</smiles>

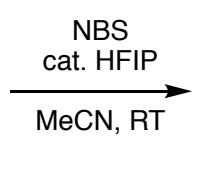<smiles>OCCc1ccc(Br)c2ccccc12</smiles>

S1

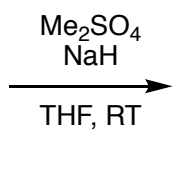

$\mathrm{MeO}$<smiles>COCCc1ccc(Br)c2ccccc12</smiles>

1D 
To a solution of 2-(naphthalen-1-yl)ethan-1-ol (1.72 g, $10 \mathrm{mmol}, 1.0$ equiv) in $\mathrm{MeCN}(5.0 \mathrm{~mL})$ were added $N$-bromosuccinimide (NBS: $1.96 \mathrm{~g}, 11 \mathrm{mmol}, 1.1$ equiv) and 1,1,1,3,3,3-hexafluoro2-propanol (HFIP: $35 \mu \mathrm{L}, 0.3 \mathrm{mmol}, 3 \mathrm{~mol} \%$ ). After stirring the mixture overnight, the solvent was evaporated in vacuo. Purification by Isolera $^{\circledR}$ (hexane/EtOAc $=20: 1$ to 2:1) afforded 2-(4-bromonaphthalen-1-yl)ethan-1-ol (S1: $2.26 \mathrm{~g}, 9.0 \mathrm{mmol}, 90 \%$ yield) as a brown oil.

To a round-bottom flask containing $\mathbf{S 1}(542 \mathrm{mg}, 2.16 \mathrm{mmol}, 1.0$ equiv) and dimethyl sulfate (306 $\mu \mathrm{L}, 3.24 \mathrm{mmol}, 1.5$ equiv) in THF $(5.0 \mathrm{~mL})$ was added sodium hydride (60\% oil dispersion; $173 \mathrm{mg}$, $4.31 \mathrm{mmol}, 2.0$ equiv) at room temperature and then the mixture was stirred for $12 \mathrm{~h}$. The reaction was quenched with water at $0{ }^{\circ} \mathrm{C}$ and extracted three times with EtOAc. The combined organic layer was dried over $\mathrm{Na}_{2} \mathrm{SO}_{4}$, filtrated, and concentrated in vacuo. The residue was purified by Isolera ${ }^{\circledR}$ (hexane/EtOAc $=50: 1$ to 5:1) to afford 1-bromo-4-(2-methoxyethyl)naphthalene (1D: $442 \mathrm{mg}, 1.67$ mmol, 77\% yield) as a colorless oil. ${ }^{1} \mathrm{H}$ NMR $\left(400 \mathrm{MHz}, \mathrm{CDCl}_{3}\right) \delta 8.27(\mathrm{~d}, J=7.6 \mathrm{~Hz}, 1 \mathrm{H}), 8.03(\mathrm{~d}, J$ $=8.0 \mathrm{~Hz}, 1 \mathrm{H}), 7.68(\mathrm{~d}, J=7.2 \mathrm{~Hz}, 1 \mathrm{H}), 7.60-7.52(\mathrm{~m}, 2 \mathrm{H}), 7.19(\mathrm{~d}, J=8.0 \mathrm{~Hz}, 1 \mathrm{H}), 3.69$ (t, $J=7.2$ $\mathrm{Hz}, 2 \mathrm{H}), 3.36(\mathrm{~s}, 3 \mathrm{H}), 3.31$ (t, $J=7.2 \mathrm{~Hz}, 2 \mathrm{H}) ;{ }^{13} \mathrm{C} \mathrm{NMR}\left(101 \mathrm{MHz}, \mathrm{CDCl}_{3}\right) \delta 135.0,133.2,132.0$, 129.5, 127.9, 127.2, 126.9, 126.7, 124.1, 121.5, 72.6, 58.7, 33.0; HRMS (DART) $\mathrm{m} / \mathrm{z}$ calcd for $\mathrm{C}_{13} \mathrm{H}_{17} \mathrm{ONBr}\left[\mathrm{M}+\mathrm{NH}_{4}\right]^{+}: 282.0488$ found 282.0480 .
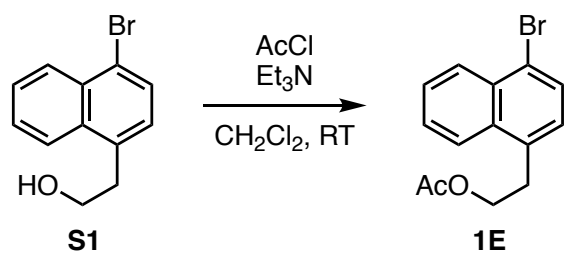

To a round-bottom flask containing $\mathbf{S} 1(489 \mathrm{mg}, 1.95 \mathrm{mmol}, 1.0$ equiv) and triethylamine (542 $\mu \mathrm{L}$, 3.89 mmol, 2.0 equiv) in $\mathrm{CH}_{2} \mathrm{Cl}_{2}(5.0 \mathrm{~mL})$ was added acetyl chloride (207 $\mu \mathrm{L}, 2.92 \mathrm{mmol}, 1.5$ equiv) at room temperature and then the mixture was stirred at room temperature for $12 \mathrm{~h}$. The reaction was quenched with water at $0{ }^{\circ} \mathrm{C}$ and extracted three times with $\mathrm{CH}_{2} \mathrm{Cl}_{2}$. The combined organic layer was dried over $\mathrm{Na}_{2} \mathrm{SO}_{4}$, filtrated, and concentrated in vacuo. The residue was purified by Isolera ${ }^{\circledR}$ (hexane/EtOAc $=50: 1$ to 5:1) to afford 2-(4-bromonaphthalen-1-yl)ethyl acetate (1E: $501 \mathrm{mg}, 1.71$ mmol, $88 \%$ yield) as a colorless oil. ${ }^{1} \mathrm{H}$ NMR $\left(400 \mathrm{MHz}, \mathrm{CDCl}_{3}\right) \delta 8.32-8.26(\mathrm{~m}, 1 \mathrm{H}), 8.10-8.05(\mathrm{~m}$, $1 \mathrm{H}), 7.72(\mathrm{~d}, J=8.0 \mathrm{~Hz}, 1 \mathrm{H}), 7.63-7.56(\mathrm{~m}, 2 \mathrm{H}), 7.21(\mathrm{~d}, J=8.0 \mathrm{~Hz}, 1 \mathrm{H}), 4.39$ (t, $J=7.6 \mathrm{~Hz}, 2 \mathrm{H})$, $3.38(\mathrm{t}, J=7.6 \mathrm{~Hz}, 2 \mathrm{H}), 2.04(\mathrm{~s}, 3 \mathrm{H}) ;{ }^{13} \mathrm{C} \mathrm{NMR}\left(101 \mathrm{MHz}, \mathrm{CDCl}_{3}\right) \delta 170.9,133.8,133.1,132.0,129.5$, $127.9,127.2,127.0,126.9,124.0,121.9,64.0,31.9,20.9$; HRMS (DART) $m / z$ calcd for $\mathrm{C}_{14} \mathrm{H}_{17} \mathrm{O}_{2} \mathrm{NBr}$ $\left[\mathrm{M}+\mathrm{NH}_{4}\right]^{+}: 310.0437$ found 310.0429 . 


\section{Synthesis of 1F}

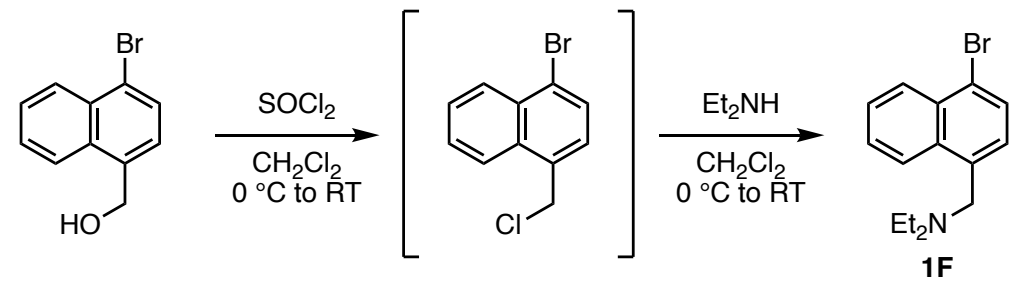

To a round-bottom flask containing (4-bromonaphthalen-1-yl)methanol (296 mg, $1.25 \mathrm{mmol}, 1.0$ equiv) in $\mathrm{CH}_{2} \mathrm{Cl}_{2}(5.0 \mathrm{~mL})$ was added thionyl chloride $\left(272 \mu \mathrm{L}, 3.75 \mathrm{mmol}, 3.0\right.$ equiv) at $0{ }^{\circ} \mathrm{C}$. After stirring at room temperature for $3 \mathrm{~h}$, the solution was concentrated in vacuo. To the resulted mixture were added $\mathrm{CH}_{2} \mathrm{Cl}_{2}(5.0 \mathrm{~mL})$, and then diethylamine (776 $\mu \mathrm{L}, 7.50 \mathrm{mmol}, 6.0$ equiv) at $0{ }^{\circ} \mathrm{C}$. After stirring the mixture at room temperature for several hours with monitoring the reaction progress by TLC, the reaction mixture was quenched with $\mathrm{NaHCO}_{3}$ aq. and extracted three times with $\mathrm{CH}_{2} \mathrm{Cl}_{2}$. The combined organic layer was dried over $\mathrm{Na}_{2} \mathrm{SO}_{4}$, filtrated, and concentrated in vacuo. Purification by $\quad$ Isolera $^{\circledR} \quad$ (hexane/EtOAc $\quad=\quad 19: 1$ to $2: 1$ ) afforded $N$-((4-bromonaphthalen-1-yl)methyl)- $N$-ethylethanamine (1F: $248 \mathrm{mg}, 850 \mu \mathrm{mol}, 68 \%$ yield in 2 steps) as a yellow oil. ${ }^{1} \mathrm{H} \mathrm{NMR}\left(400 \mathrm{MHz}, \mathrm{CDCl}_{3}\right) \delta 8.35(\mathrm{~d}, J=8.0 \mathrm{~Hz}, 1 \mathrm{H}), 8.26(\mathrm{~d}, J=8.0 \mathrm{~Hz}, 1 \mathrm{H})$, $7.70(\mathrm{~d}, J=8.0 \mathrm{~Hz}, 1 \mathrm{H}), 7.61-7.50(\mathrm{~m}, 2 \mathrm{H}), 7.35$ (d, $J=8.0 \mathrm{~Hz}, 1 \mathrm{H}), 3.93(\mathrm{~s}, 2 \mathrm{H}), 2.57$ (d, $J=7.6 \mathrm{~Hz}$, $4 \mathrm{H}), 1.06(\mathrm{~d}, J=7.6 \mathrm{~Hz}, 6 \mathrm{H}) ;{ }^{13} \mathrm{C} \mathrm{NMR}\left(101 \mathrm{MHz}, \mathrm{CDCl}_{3}\right) \delta 135.9,133.7,132.0,129.3,127.5,127.4$, 126.9, 126.4, 125.0, 122.0, 55.8, 46.9, 11.5; HRMS (DART) $\mathrm{m} / z$ calcd for $\mathrm{C}_{15} \mathrm{H}_{19} \mathrm{NBr}[\mathrm{M}+\mathrm{H}]^{+}$: 292.0695 found 292.0688 .

\section{Synthesis of $1 \mathrm{~J}^{[5]}$}

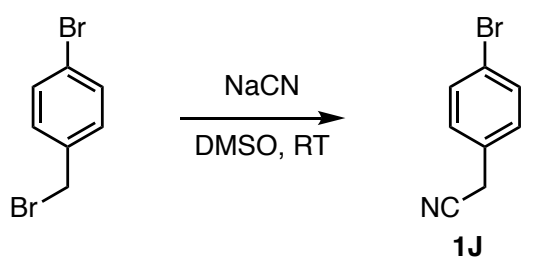

To a round-bottom flask with 1-bromo-4-(bromomethyl)benzene ( $375 \mathrm{mg}, 1.50 \mathrm{mmol}, 1.0$ equiv), were added sodium cyanide $(80.9 \mathrm{mg}, 1.65 \mathrm{mmol}, 1.1$ equiv) and DMSO $(5.0 \mathrm{~mL})$. After stirring the mixture for several hours at room temperature with monitoring reaction progress by TLC, the reaction was quenched with $\mathrm{NaHCO}_{3}$ aq. and extracted three times with EtOAc. The combined organic layer was dried over $\mathrm{Na}_{2} \mathrm{SO}_{4}$, filtrated, and concentrated in vacuo. The residue was purified by Isolera ${ }^{\circledR}$ (hexane/EtOAc $=40: 1$ to 4:1) to afford 2-(4-bromophenyl)acetonitrile (1J: $221 \mathrm{mg}, 1.13 \mathrm{mmol}, 75 \%$ yield) as a white solid. The spectra are in accordance with those of the compounds reported in the literature. 


\section{Synthesis of $1 K^{[6]}$}

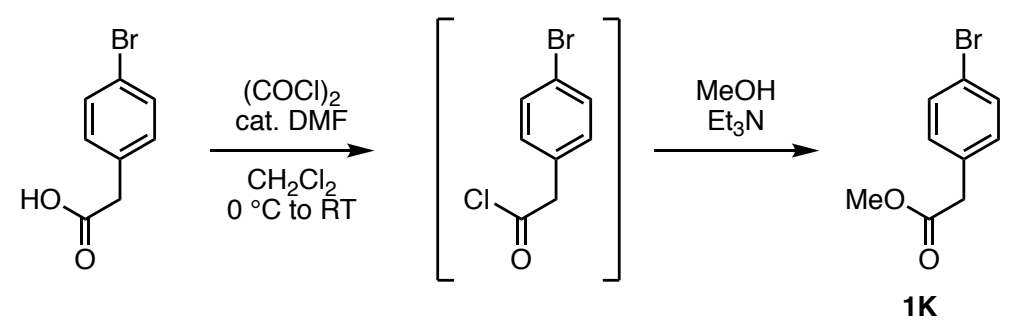

To a solution of 2-(4-bromophenyl)acetic acid (1.08 g, $5.00 \mathrm{mmol}, 1.0$ equiv) in $\mathrm{CH}_{2} \mathrm{Cl}_{2}(10 \mathrm{~mL})$ were added oxalyl chloride ( $643 \mu \mathrm{L}, 7.50 \mathrm{mmol}, 1.5$ equiv) and one portion of DMF. After stirring at room temperature for $1.5 \mathrm{~h}$, the solution was concentrated in vacuo. To the resulted mixture were added methanol (1.6 mL), $\mathrm{CH}_{2} \mathrm{Cl}_{2}(10 \mathrm{~mL})$, and then triethylamine (1.39 mL, $10.0 \mathrm{mmol}, 2.0$ equiv) slowly at $0{ }^{\circ} \mathrm{C}$. After stirring the mixture for several hours with monitoring the reaction progress by TLC, the reaction mixture was quenched with $\mathrm{NaHCO}_{3}$ aq. and extracted three times with $\mathrm{CH}_{2} \mathrm{Cl}_{2}$. The combined organic layer was dried over $\mathrm{Na}_{2} \mathrm{SO}_{4}$, filtrated, and concentrated in vacuo. Purification by Isolera ${ }^{\circledR}$ (hexane/EtOAc $=50: 1$ to 5:1) afforded methyl 2-(4-bromophenyl)acetate (1K: $1.08 \mathrm{~g}, 4.71$ mmol, 94\% yield) as a colorless oil. The spectra are in accordance with those of the compounds reported in the literature.

\section{Synthesis of $1 L^{[7]}$}

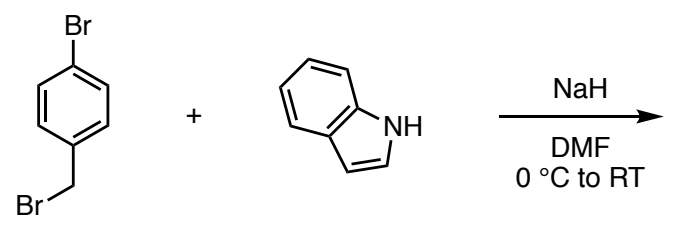

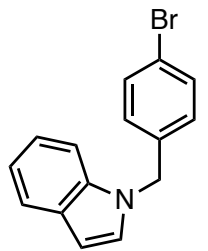

1L

To a solution of indole (234 mg, $2.00 \mathrm{mmol}, 1.0$ equiv) in DMF (15 mL) was added sodium hydride (60\% oil dispersion; $88.0 \mathrm{mg}, 2.20 \mathrm{mmol}, 1.1$ equiv) at $0{ }^{\circ} \mathrm{C}$. After stirring for $30 \mathrm{~min}$ at $0{ }^{\circ} \mathrm{C}$, 1-bromo-4-(bromomethyl)benzene (600 mg, $3.60 \mathrm{mmol}, 1.2$ equiv) was added and then the mixture was stirred at room temperature for $12 \mathrm{~h}$. The reaction was quenched with water at $0{ }^{\circ} \mathrm{C}$ and extracted three times with EtOAc. The combined organic layer was dried over $\mathrm{Na}_{2} \mathrm{SO}_{4}$, filtrated, and concentrated in vacuo. The residue was purified by Isolera ${ }^{\circledR}$ (hexane/EtOAc $=40: 1$ to $4: 1$ ) to afford 1-(4-bromobenzyl)-1H-indole (1L: $395 \mathrm{mg}, 1.38 \mathrm{mmol}, 69 \%$ yield) as a white solid. The spectra are in accordance with those of the compounds reported in the literature. 


\section{Synthesis of $1 \mathrm{M}$}

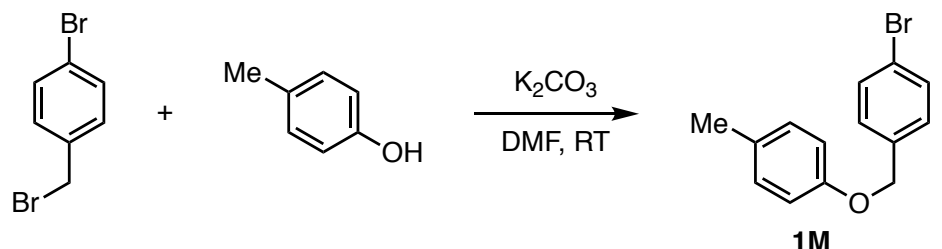

To a round-bottom flask with 1-bromo-4-(bromomethyl)benzene $(2.50 \mathrm{~g}, 10.0 \mathrm{mmol}, 1.0$ equiv) were added $p$-cresol (1.26 mL, $12.0 \mathrm{mmol}, 1.2$ equiv), potassium carbonate (4.15 g, $30.0 \mathrm{mmol}, 3.0$ equiv), and DMF (10 mL). After stirring the mixture for several hours at room temperature with monitoring reaction progress by TLC, the reaction was quenched with $\mathrm{NaHCO}_{3}$ aq. and extracted three times with EtOAc. The combined organic layer was dried over $\mathrm{Na}_{2} \mathrm{SO}_{4}$, filtrated, and concentrated in vacuo. Purification by recrystallization (hexane $/ \mathrm{CH}_{2} \mathrm{Cl}_{2}$ ) afforded 1-bromo-4-((p-tolyloxy)methyl)benzene (1M: $2.49 \mathrm{~g}, 8.97 \mathrm{mmol}, 90 \%$ yield) as a white solid. ${ }^{1} \mathrm{H}$ NMR $\left(400 \mathrm{MHz}, \mathrm{CDCl}_{3}\right) \delta 7.50(\mathrm{~d}, J=8.0 \mathrm{~Hz}, 2 \mathrm{H}), 7.30(\mathrm{~d}, J=8.0 \mathrm{~Hz}, 2 \mathrm{H}), 7.08(\mathrm{~d}, J=8.8 \mathrm{~Hz}, 2 \mathrm{H})$, $6.84(\mathrm{~d}, J=8.8 \mathrm{~Hz}, 2 \mathrm{H}), 4.99$ (s, 2H), 2.29 (s, 3H); ${ }^{13} \mathrm{C} \mathrm{NMR}\left(101 \mathrm{MHz}, \mathrm{CDCl}_{3}\right) \delta 156.3,136.3,131.6$, 130.4, 129.9, 129.0, 121.7, 114.6, 69.3, 20.5; HRMS (DART) $\mathrm{m} / z$ calcd for $\mathrm{C}_{14} \mathrm{H}_{17} \mathrm{ONBr}\left[\mathrm{M}+\mathrm{NH}_{4}\right]^{+}$: 294.0488 found 294.0479 .

\section{Synthesis of $1 T^{[8]}$}
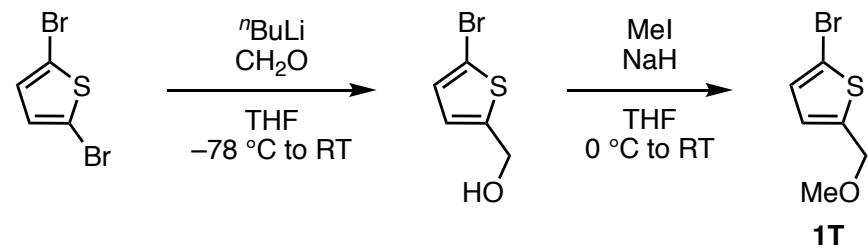

A $100-\mathrm{mL}$ two-necked flask, containing a magnetic stirring bar, was dried with a heat-gun in vacuo and filled with $\mathrm{N}_{2}$ after cooling to room temperature. To this flask were added 2,5-dibromothiophene (726 mg, $3.00 \mathrm{mmol}, 1.0$ equiv) and THF (20 mL) under a stream of $\mathrm{N}_{2}$. The mixture was cooled at $78{ }^{\circ} \mathrm{C}$ and then $n$-butyllithium (1.4 M in hexane: $4.29 \mathrm{~mL}, 6.00 \mathrm{mmol}, 1.0$ equiv) was slowly added. The mixture was stirred at $-78{ }^{\circ} \mathrm{C}$ for $30 \mathrm{~min}$. To this mixture was slowly added paraformaldehyde ( $99.1 \mathrm{mg}, 3.30 \mathrm{mmol}, 1.1$ equiv) in THF $(10 \mathrm{~mL})$. The mixture was further stirred at room temperature for $3 \mathrm{~h}$. The reaction was quenched by adding $\mathrm{NH}_{4} \mathrm{Cl}$ aq. The mixture was extracted three times with EtOAc. The combined organic layer was dried over $\mathrm{Na}_{2} \mathrm{SO}_{4}$, filtrated, and concentrated in vacuo to afford (5-bromothiophen-2-yl)methanol. The crude material was used for the next step without further purification.

To a round-bottom flask containing the crude material in THF $(15 \mathrm{~mL})$ was added sodium hydride (60\% oil dispersion; $132 \mathrm{mg}, 3.30 \mathrm{mmol}, 1.1$ equiv) at $0{ }^{\circ} \mathrm{C}$. After stirring for $30 \mathrm{~min}$ at $0{ }^{\circ} \mathrm{C}$, iodomethane ( $224 \mu \mathrm{L}, 3.60 \mathrm{mmol}, 1.2$ equiv) was added and then the mixture was stirred at room 
temperature for $12 \mathrm{~h}$. The reaction was quenched with water at $0{ }^{\circ} \mathrm{C}$ and extracted three times with EtOAc. The combined organic layer was dried over $\mathrm{Na}_{2} \mathrm{SO}_{4}$, filtrated, and concentrated in vacuo. The residue was purified by Isolera $^{\circledR}$ (hexane/EtOAc $=50: 1$ to 4:1) to afford 2-bromo-5-(methoxymethyl)thiophene (1T: $502 \mathrm{mg}, 2.42 \mathrm{mmol}, 81 \%$ yield in 2 steps) as a colorless oil. The spectra are in accordance with those of the compounds reported in the literature.

\section{Synthesis of $1 \mathrm{~W}$ and $1 \mathrm{X}$}
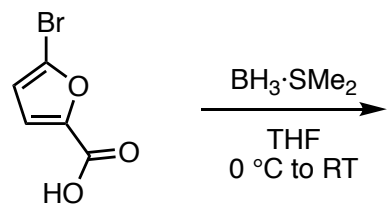<smiles>OCc1ccc(Br)o1</smiles>

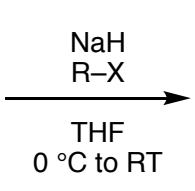<smiles>OCc1ccc(Br)o1</smiles>

To a solution of 5-bromo-2-furoic acid (1.0 equiv) in THF $(0.20 \mathrm{M})$ was added dimethyl sulfide borane (2.0 equiv) at $0{ }^{\circ} \mathrm{C}$. After stirring the mixture for several hours at room temperature with monitoring reaction progress by TLC, the reaction was quenched with water at $0{ }^{\circ} \mathrm{C}$ and extracted three times with $\mathrm{Et}_{2} \mathrm{O}$. The combined organic layer was washed with $\mathrm{NaHCO}_{3}$ aq. and dried over $\mathrm{MgSO}_{4}$, filtrated, and concentrated in vacuo to afford (5-bromofuran-2-yl)methanol. The crude material was used for the next step without further purification.

To a round-bottom flask containing the crude material in THF $(0.20 \mathrm{M})$ was added sodium hydride (60\% oil dispersion; 1.0 equiv) at $0{ }^{\circ} \mathrm{C}$. After stirring for $30 \mathrm{~min}$ at $0{ }^{\circ} \mathrm{C}$, alkyl halide (1.0 equiv) was added and then the mixture was stirred at room temperature for $6 \mathrm{~h}$. The reaction was quenched with water at $0{ }^{\circ} \mathrm{C}$ and extracted three times with EtOAc. The combined organic layer was dried over $\mathrm{MgSO}_{4}$, filtrated, and concentrated in vacuo. The residue was purified by Isolera ${ }^{\circledR}$ (hexane/EtOAc $=$ 99:1 to $3: 2$ ) to afford the corresponding bromofuran.

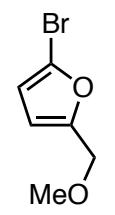

\section{2-Bromo-5-(methoxymethyl)furan (1W)}

By using iodomethane (623 $\mu \mathrm{L}, 10.0 \mathrm{mmol}, 1.0$ equiv) as an alkyl halide, $\mathbf{1 W}$ was obtained (959 $\mathrm{mg}, 10.0 \mathrm{mmol}$ scale, $50 \%$ yield in 2 steps) as a colorless oil. ${ }^{1} \mathrm{H}$ NMR $\left(400 \mathrm{MHz}, \mathrm{CDCl}_{3}\right) \delta 6.31(\mathrm{~d}, J$ $=3.2 \mathrm{~Hz}, 1 \mathrm{H}), 6.27(\mathrm{~d}, J=3.2 \mathrm{~Hz}, 1 \mathrm{H}), 4.35(\mathrm{~s}, 2 \mathrm{H}), 3.37(\mathrm{~s}, 3 \mathrm{H}) ;{ }^{13} \mathrm{C} \mathrm{NMR}\left(101 \mathrm{MHz}, \mathrm{CDCl}_{3}\right) \delta$ 153.7, 122.1, 112.0, 111.9, 66.1, 57.9; HRMS (DART) $\mathrm{m} / \mathrm{z}$ calcd for $\mathrm{C}_{6} \mathrm{H}_{11} \mathrm{O}_{2} \mathrm{NBr}\left[\mathrm{M}+\mathrm{NH}_{4}\right]^{+}$: 207.9968 found 207.9967. 


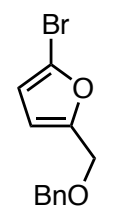

\section{2-((Benzyloxy)methyl)-5-bromofuran (1X)}

By using benzyl bromide (300 $\mu \mathrm{L}, 2.50 \mathrm{mmol}, 1.0$ equiv) as an alkyl halide, $\mathbf{1 X}$ was obtained (363.4 mg, $2.5 \mathrm{mmol}$ scale, 54\% yield in 2 steps) as a colorless oil. ${ }^{1} \mathrm{H}$ NMR (400 $\left.\mathrm{MHz}, \mathrm{CDCl}_{3}\right) \delta$ 7.38-7.27 (m, 5H), $6.30(\mathrm{~d}, J=3.2 \mathrm{~Hz}, 1 \mathrm{H}), 6.27(\mathrm{~d}, J=3.2 \mathrm{~Hz}, 1 \mathrm{H}), 4.56(\mathrm{~s}, 2 \mathrm{H}), 4.44(\mathrm{~s}, 2 \mathrm{H}) ;{ }^{13} \mathrm{C}$ NMR $\left(101 \mathrm{MHz}, \mathrm{CDCl}_{3}\right) \delta 153.7,137.5,128.4,127.8,127.7,122.0,112.1,111.9,71.9,63.5 ;$ HRMS (DART) $m / z$ calcd for $\mathrm{C}_{12} \mathrm{H}_{15} \mathrm{O}_{2} \mathrm{NBr}\left[\mathrm{M}+\mathrm{NH}_{4}\right]^{+}: 284.0281$ found 284.0278 . 


\section{Pd-Catalyzed Dearomative Three-Component Reaction of Bromoarenes}

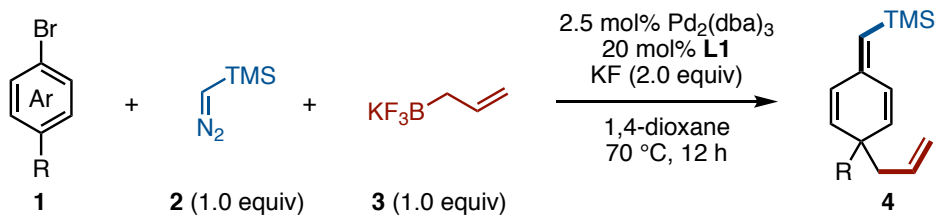

\section{General Procedure}

To a $25-\mathrm{mL}$ glass vessel equipped with a screw cap containing a magnetic stirring bar and potassium fluoride ( $46.5 \mathrm{mg}, 0.80 \mathrm{mmol}, 2.0$ equiv) was dried with a heat-gun in vacuo and filled with $\mathrm{N}_{2}$ after cooling to room temperature. To this vessel were added bromoarene 1 ( $0.40 \mathrm{mmol}, 1.0$ equiv), $\mathrm{Pd}_{2}(\mathrm{dba})_{3} \cdot \mathrm{CHCl}_{3}(10.4 \mathrm{mg}, 0.010 \mathrm{mmol}, 2.5 \mathrm{~mol} \%)$, 4-(dimethylamino)phenyldiphenylphosphine (L1: $24.4 \mathrm{mg}, 0.080 \mathrm{mmol}, 20 \mathrm{~mol} \%$ ), potassium allyltrifluoroborate (3: $59.2 \mathrm{mg}, 0.40 \mathrm{mmol}, 1.0$ equiv). The vessel was placed under vacuum and refilled $\mathrm{N}_{2}$ gas three times, and then added 1,4-dioxane (2.0 $\mathrm{mL}$ ). The mixture was stirred for several minutes until the color of the solution turned to yellow. A solution of (trimethylsilyl)diazomethane (2, 2.0 M in $\mathrm{Et}_{2} \mathrm{O}: 200 \mu \mathrm{L}, 0.40 \mathrm{mmol}, 1.0$ equiv) was then added slowly. The vessel was sealed with a screw cap and then heated at $70{ }^{\circ} \mathrm{C}$ for $12 \mathrm{~h}$ with stirring. After cooling the reaction mixture to room temperature, the mixture was passed through a short alumina pad with hexane as an eluent. The filtrate was concentrated in vacuo. The yield of $\mathbf{4}$ was determined by ${ }^{1} \mathrm{H}$ NMR analysis using $\mathrm{CH}_{2} \mathrm{Br}_{2}$ as an internal standard (generally ${ }^{1} \mathrm{H}$ NMR signal at $\mathrm{C} 1$ position was used to determine the yield). The residue was purified by Isolera ${ }^{\circledR}$ with a basic alumina column cartridge (hexane) to afford the corresponding dearomatized product 4.

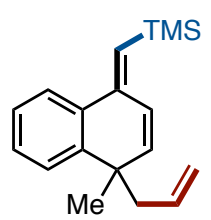

\section{(E)-((4-Allyl-4-methylnaphthalen-1(4H)-ylidene)methyl)trimethylsilane (4A)}

${ }^{1} \mathrm{H}$ NMR yield of $\mathbf{4 A}$ was $84 \%\left({ }^{1} \mathrm{H}\right.$ NMR peak at $6.27 \mathrm{ppm}(\mathrm{s}, 1 \mathrm{H})$ was used). Purification by Isolera $^{\circledR}$ with a basic alumina column cartridge (hexane) afforded $4 \mathbf{A}(73.0 \mathrm{mg}, 272 \mu \mathrm{mol}, 68 \%$ yield) as a colorless oil. A part of product underwent to decomposition (rearomatization) during purification. ${ }^{1} \mathrm{H}$ NMR $\left(400 \mathrm{MHz}, \mathrm{CDCl}_{3}\right) \delta 7.82(\mathrm{~d}, J=8.0 \mathrm{~Hz}, 1 \mathrm{H}), 7.38(\mathrm{~d}, J=8.0 \mathrm{~Hz}, 1 \mathrm{H}), 7.30(\mathrm{t}, J=8.0 \mathrm{~Hz}$, 1H), $7.23(\mathrm{t}, J=8.0 \mathrm{~Hz}, 1 \mathrm{H}), 6.65(\mathrm{~d}, J=10.0 \mathrm{~Hz}, 1 \mathrm{H}), 6.27(\mathrm{~s}, 1 \mathrm{H}), 5.78(\mathrm{~d}, J=10.0 \mathrm{~Hz}, 1 \mathrm{H}), 5.55-$ $5.41(\mathrm{~m}, 1 \mathrm{H}), 4.94-4.84(\mathrm{~m}, 2 \mathrm{H}), 2.58(\mathrm{dd}, J=13.6,7.2 \mathrm{~Hz}, 1 \mathrm{H}), 2.37(\mathrm{dd}, J=13.6,7.2 \mathrm{~Hz}, 1 \mathrm{H}), 1.41$ (s, 3H), 0.25 (s, 9H); ${ }^{13} \mathrm{C}$ NMR (101 MHz, $\left.\mathrm{CDCl}_{3}\right) \delta 144.1,141.9,137.3,134.6,133.2,127.8,126.4$, 126.3, 126.0, 123.3, 123.0, 117.1, 48.6, 40.3, 29.8, 0.4; HRMS (DART) $m / z$ calcd for $\mathrm{C}_{18} \mathrm{H}_{25} \mathrm{Si}[\mathrm{M}+$ $\mathrm{H}]^{+}: 269.1720$ found 269.1714 . 


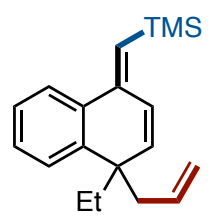

\section{(E)-((4-Allyl-4-ethylnaphthalen-1(4H)-ylidene)methyl)trimethylsilane (4B)}

${ }^{1} \mathrm{H}$ NMR yield of 4B was $87 \%\left({ }^{1} \mathrm{H}\right.$ NMR peak at $6.31 \mathrm{ppm}(\mathrm{s}, 1 \mathrm{H})$ was used). Purification by Isolera ${ }^{\circledR}$ with a basic alumina column cartridge (hexane) afforded 4B (81.8 mg, $292 \mu \mathrm{mol}, 73 \%$ yield) as a colorless oil. A part of product underwent to decomposition (rearomatization) during purification. ${ }^{1} \mathrm{H}$ NMR (400 MHz, $\left.\mathrm{CDCl}_{3}\right) \delta 7.89(\mathrm{~d}, J=8.0 \mathrm{~Hz}, 1 \mathrm{H}), 7.41-7.31(\mathrm{~m}, 2 \mathrm{H}), 7.27(\mathrm{t}, J=8.0 \mathrm{~Hz}, 1 \mathrm{H})$, $6.83(\mathrm{~d}, J=10.4 \mathrm{~Hz}, 1 \mathrm{H}), 6.31(\mathrm{~s}, 1 \mathrm{H}), 5.71(\mathrm{~d}, J=10.4 \mathrm{~Hz}, 1 \mathrm{H}), 5.59-5.46(\mathrm{~m}, 1 \mathrm{H}), 5.00-4.87$ (m, 2H), $2.68(\mathrm{dd}, J=10.0,7.2 \mathrm{~Hz}, 1 \mathrm{H}), 2.43(\mathrm{dd}, J=10.0,7.2 \mathrm{~Hz}, 1 \mathrm{H}), 1.99$ (dq, $J=14.4,7.6 \mathrm{~Hz}, 1 \mathrm{H})$, $1.71(\mathrm{dq}, J=14.4,7.6 \mathrm{~Hz}, 1 \mathrm{H}), 0.64(\mathrm{t}, J=7.6 \mathrm{~Hz}, 3 \mathrm{H}), 0.31(\mathrm{~s}, 9 \mathrm{H}) ;{ }^{13} \mathrm{C} \mathrm{NMR}\left(101 \mathrm{MHz}, \mathrm{CDCl}_{3}\right) \delta$ 144.4, 139.8, 135.8, 134.6, 128.3, 127.8, 126.2, 125.9, 123.2, 122.9, 116.9, 48.1, 45.1, 35.7, 9.0, 0.4 (one peak is missing due to overlapping); HRMS (DART) $\mathrm{m} / z$ calcd for $\mathrm{C}_{19} \mathrm{H}_{27} \mathrm{Si}[\mathrm{M}+\mathrm{H}]^{+}: 283.1877$ found 283.1871 .

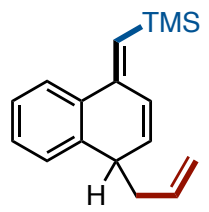

\section{(E)-((4-Allylnaphthalen-1(4H)-ylidene)methyl)trimethylsilane (4C)}

${ }^{1} \mathrm{H}$ NMR yield of $4 \mathrm{C}$ was $79 \%\left({ }^{1} \mathrm{H}\right.$ NMR peak at $6.24 \mathrm{ppm}(\mathrm{s}, 1 \mathrm{H})$ was used). Purification by Isolera ${ }^{\circledR}$ with a basic alumina column cartridge (hexane) afforded 4C (73.8 mg, $290 \mu \mathrm{mol}, 73 \%$ yield) as a colorless oil. A part of product underwent to decomposition (rearomatization) during purification. ${ }^{1} \mathrm{H}$ NMR (400 MHz, CDCl $) \delta 7.78(\mathrm{~d}, J=6.4 \mathrm{~Hz}, 1 \mathrm{H}), 7.30-7.21(\mathrm{~m}, 3 \mathrm{H}), 6.69(\mathrm{~d}, J=10.0 \mathrm{~Hz}, 1 \mathrm{H})$, $6.24(\mathrm{~s}, 1 \mathrm{H}), 6.07(\mathrm{dd}, J=10.0,4.8 \mathrm{~Hz}, 1 \mathrm{H}), 5.77-5.65(\mathrm{~m}, 1 \mathrm{H}), 5.01-4.94(\mathrm{~m}, 2 \mathrm{H}), 3.70-3.59(\mathrm{~m}$, 1H), 2.68-2.49 (m, 1H), 2.45-2.36 (m, 1H), $0.23(\mathrm{~s}, 9 \mathrm{H}) ;{ }^{13} \mathrm{C} \mathrm{NMR}\left(101 \mathrm{MHz}, \mathrm{CDCl}_{3}\right) \delta 144.3,137.9$, 135.3, 134.0, 131.5, 128.3, 128.1, 127.5, 126.3, 123.4, 123.1, 117.0, 42.8, 40.3, 0.3; HRMS (DART) $m / z$ calcd for $\mathrm{C}_{17} \mathrm{H}_{23} \mathrm{Si}[\mathrm{M}+\mathrm{H}]^{+}: 255.1564$ found 255.1561 .

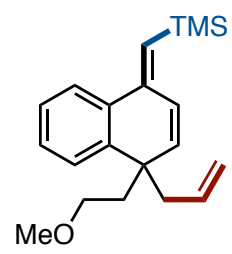

(E)-((4-Allyl-4-(2-methoxyethyl)naphthalen-1(4H)-ylidene)methyl)trimethylsilane (4D) 
${ }^{1} \mathrm{H}$ NMR yield of 4D was $79 \%\left({ }^{1} \mathrm{H}\right.$ NMR peak at $6.26 \mathrm{ppm}(\mathrm{s}, 1 \mathrm{H})$ was used). Purification by Isolera ${ }^{\circledR}$ with a basic alumina column cartridge (hexane) afforded 4D (62.8 mg, $201 \mu \mathrm{mol}, 50 \%$ yield) as a colorless oil. A part of product underwent to decomposition (rearomatization) during purification. ${ }^{1} \mathrm{H}$ NMR (400 MHz, $\left.\mathrm{CDCl}_{3}\right) \delta 7.80(\mathrm{~d}, J=8.0 \mathrm{~Hz}, 1 \mathrm{H}), 7.37$ (d, $\left.J=8.0 \mathrm{~Hz}, 1 \mathrm{H}\right), 7.34-7.19(\mathrm{~m}, 2 \mathrm{H})$, $6.71(\mathrm{~d}, J=10.0 \mathrm{~Hz}, 1 \mathrm{H}), 6.26(\mathrm{~s}, 1 \mathrm{H}), 5.73(\mathrm{~d}, J=10.0 \mathrm{~Hz}, 1 \mathrm{H}), 5.50-5.37(\mathrm{~m}, 1 \mathrm{H}), 4.95-4.83(\mathrm{~m}$, $2 \mathrm{H}), 3.15(\mathrm{~s}, 3 \mathrm{H}), 3.14-3.10(\mathrm{~m}, 1 \mathrm{H}), 2.95-2.85(\mathrm{~m}, 1 \mathrm{H}), 2.60(\mathrm{dd}, J=14.0,7.6 \mathrm{~Hz}, 1 \mathrm{H}), 2.39$ (dd, $J=$ 14.0, 7.6 Hz, 1H), 2.32-2.19 (m, 1H), 2.00-1.88 (m, 1H), $0.23(\mathrm{~s}, 9 \mathrm{H}) ;{ }^{13} \mathrm{C} \mathrm{NMR}\left(101 \mathrm{MHz}, \mathrm{CDCl}_{3}\right) \delta$ 143.9, 139.3, 135.3, 134.0, 133.8, 128.0, 127.9, 126.22, 126.18, 123.7, 123.3, 117.5, 69.5, 58.5, 48.6, 42.6, 42.0, 0.3; HRMS (DART) $\mathrm{m} / z$ calcd for $\mathrm{C}_{20} \mathrm{H}_{29} \mathrm{OSi}[\mathrm{M}+\mathrm{H}]^{+}: 313.1982$ found 313.1977.

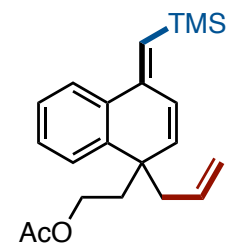

\section{(E)-2-(1-Allyl-4-((trimethylsilyl)methylene)-1,4-dihydronaphthalen-1-yl)ethyl acetate (4E)}

${ }^{1} \mathrm{H}$ NMR yield of $\mathbf{4 E}$ was $86 \%\left({ }^{1} \mathrm{H}\right.$ NMR peak at $6.27 \mathrm{ppm}(\mathrm{s}, 1 \mathrm{H})$ was used). Purification by Isolera $^{\circledR}$ with a basic alumina column cartridge (hexane) afforded $4 \mathrm{E}(60.2 \mathrm{mg}, 177 \mu \mathrm{mol}, 44 \%$ yield) as a colorless oil. A part of product underwent to decomposition (rearomatization) during purification. ${ }^{1} \mathrm{H}$ NMR $\left(400 \mathrm{MHz}, \mathrm{CDCl}_{3}\right) \delta 7.80(\mathrm{~d}, J=7.6 \mathrm{~Hz}, 1 \mathrm{H}), 7.35$ (d, $\left.J=7.6 \mathrm{~Hz}, 1 \mathrm{H}\right), 7.29$ (t, $J=7.6 \mathrm{~Hz}$, 1H), 7.21 (t, $J=7.6 \mathrm{~Hz}, 1 \mathrm{H}), 6.73(\mathrm{~d}, J=10.0 \mathrm{~Hz}, 1 \mathrm{H}), 6.27$ (s, 1H), 5.70 (d, $J=10.0 \mathrm{~Hz}, 1 \mathrm{H}), 5.50$ $5.38(\mathrm{~m}, 1 \mathrm{H}), 4.94-4.85(\mathrm{~m}, 2 \mathrm{H}), 3.90$ (ddd, $J=10.8,9.6,5.6 \mathrm{~Hz}, 1 \mathrm{H}), 3.64$ (ddd, $J=10.8,9.6,5.6 \mathrm{~Hz}$, 1H), 2.59 (dd, $J=14.0,8.4 \mathrm{~Hz}, 1 \mathrm{H}), 2.38$ (dd, $J=14.0,8.4 \mathrm{~Hz}, 1 \mathrm{H}), 2.31$ (ddd, $J=10.8,9.6,5.6 \mathrm{~Hz}$, 1H), 1.96 (ddd, $J=10.8,9.6,5.6 \mathrm{~Hz}, 1 \mathrm{H}), 1.88(\mathrm{~s}, 3 \mathrm{H}), 0.23(\mathrm{~s}, 9 \mathrm{H}) ;{ }^{13} \mathrm{C} \mathrm{NMR}\left(101 \mathrm{MHz}, \mathrm{CDCl}_{3}\right) \delta$ 170.9, 143.6, 138.7, 134.5, 134.1, 133.6, 128.4, 128.0, 126.3, 126.1, 124.2, 123.5, 117.7, 61.8, 48.5, 42.7, 40.7, 20.9, 0.3; HRMS (DART) $\mathrm{m} / z$ calcd for $\mathrm{C}_{21} \mathrm{H}_{32} \mathrm{O}_{2} \mathrm{NSi}\left[\mathrm{M}+\mathrm{NH}_{4}\right]^{+}: 358.2197$ found 358.2190 .

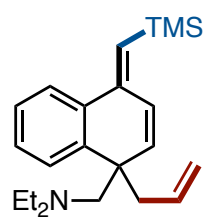

(E)-1-(1-Allyl-4-((trimethylsilyl)methylene)-1,4-dihydronaphthalen-1-yl)- $N, N$-diethylmethanami ne (4F)

${ }^{1} \mathrm{H}$ NMR yield of $\mathbf{4 F}$ was $81 \%\left({ }^{1} \mathrm{H}\right.$ NMR peak at $6.20 \mathrm{ppm}(\mathrm{s}, 1 \mathrm{H})$ was used). Purification by Isolera $^{\circledR}$ with a basic alumina column cartridge (hexane/EtOAc $=19: 1$ to $\left.4: 1\right)$ afforded $\mathbf{4 F}(81.2 \mathrm{mg}$, $260 \mu \mathrm{mol}, 65 \%$ yield) as a colorless oil. A part of product underwent to decomposition 
(rearomatization) during purification. ${ }^{1} \mathrm{H} \mathrm{NMR}\left(400 \mathrm{MHz}, \mathrm{CDCl}_{3}\right) \delta 7.80(\mathrm{dd}, J=7.6,1.6 \mathrm{~Hz}, 1 \mathrm{H})$, $7.38(\mathrm{dd}, J=8.0,1.6 \mathrm{~Hz}, 1 \mathrm{H}), 7.28-7.17(\mathrm{~m}, 2 \mathrm{H}), 6.70(\mathrm{~d}, J=10.0 \mathrm{~Hz}, 1 \mathrm{H}), 6.20(\mathrm{~s}, 1 \mathrm{H}), 5.88(\mathrm{~d}, J=$ $10.0 \mathrm{~Hz}, 1 \mathrm{H}), 5.49-5.37(\mathrm{~m}, 1 \mathrm{H}), 4.96-4.88(\mathrm{~m}, 1 \mathrm{H}), 4.85-4.79(\mathrm{~m}, 1 \mathrm{H}), 2.71(\mathrm{dd}, J=14.4,7.2 \mathrm{~Hz}$, 1H), 2.63 (s, 2H), 2.52 (dd, $J=14.4,7.2 \mathrm{~Hz}, 1 \mathrm{H}), 2.45-2.31$ (m, 4H), 0.85 (t, $J=7.2 \mathrm{~Hz}, 6 \mathrm{H}), 0.22$ (s, $9 \mathrm{H}) ;{ }^{13} \mathrm{C}$ NMR $\left(101 \mathrm{MHz}, \mathrm{CDCl}_{3}\right) \delta 144.7,139.8,136.1,135.0,134.4,127.4,127.3,126.7,125.9$, 123.3, 122.3, 116.7, 66.6, 48.3, 46.1, 43.9, 11.8, 0.4; HRMS (ESI) $m / z$ calcd for $\mathrm{C}_{22} \mathrm{H}_{34} \mathrm{NSi}[\mathrm{M}+\mathrm{H}]^{+}$: 340.2455 found 340.2449 .

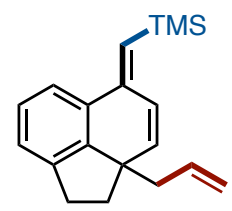

\section{(E)-((2a-Allyl-2,2a-dihydroacenaphthylen-5(1H)-ylidene)methyl)trimethylsilane (4G)}

${ }^{1} \mathrm{H}$ NMR yield of $\mathbf{4 G}$ was $78 \%\left({ }^{1} \mathrm{H}\right.$ NMR peak at $6.27 \mathrm{ppm}(\mathrm{s}, 1 \mathrm{H})$ was used). Purification by Isolera $^{\circledR}$ with a basic alumina column cartridge (hexane) afforded 4G (59.2 mg, $211 \mu \mathrm{mol}, 53 \%$ yield) as a colorless oil. A part of product underwent to decomposition (rearomatization) during purification. ${ }^{1} \mathrm{H}$ NMR $\left(400 \mathrm{MHz}, \mathrm{CDCl}_{3}\right) \delta 7.42(\mathrm{~d}, J=7.6 \mathrm{~Hz}, 1 \mathrm{H}), 7.19(\mathrm{~d}, J=7.6 \mathrm{~Hz}, 1 \mathrm{H}), 7.14(\mathrm{t}, J=7.6 \mathrm{~Hz}$, $1 \mathrm{H}), 6.58(\mathrm{~d}, J=10.0 \mathrm{~Hz}, 1 \mathrm{H}), 6.29(\mathrm{~s}, 1 \mathrm{H}), 6.19(\mathrm{~d}, J=10.0 \mathrm{~Hz}, 1 \mathrm{H}), 5.72-5.59(\mathrm{~m}, 1 \mathrm{H}), 4.98-4.87$ (m, 2H), 3.20-3.09 (m, 1H), $2.76(\mathrm{dd}, J=16.0,8.0 \mathrm{~Hz}, 1 \mathrm{H}), 2.28-2.15(\mathrm{~m}, 3 \mathrm{H}), 2.00-1.91(\mathrm{~m}, 1 \mathrm{H})$, $0.23(\mathrm{~s}, 9 \mathrm{H}) ;{ }^{13} \mathrm{C} \mathrm{NMR}\left(101 \mathrm{MHz}, \mathrm{CDCl}_{3}\right) \delta 145.9,145.4,141.1,136.4,135.0,132.8,128.3,126.9$, 124.6, 123.3, 120.4 , 117.5, 48.9, 45.1, 36.6, 30.2, 0.2; HRMS (DART) $m / z$ calcd for $\mathrm{C}_{19} \mathrm{H}_{25} \mathrm{Si}[\mathrm{M}+$ $\mathrm{H}]^{+}: 281.1720$ found 281.1715 .

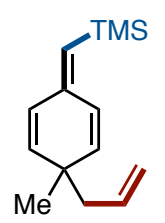

\section{((4-Allyl-4-methylcyclohexa-2,5-dien-1-ylidene)methyl)trimethylsilane (4H)}

${ }^{1} \mathrm{H}$ NMR yield of $\mathbf{4 H}$ was $70 \%\left({ }^{1} \mathrm{H}\right.$ NMR peak at $5.30 \mathrm{ppm}(\mathrm{s}, 1 \mathrm{H})$ was used). Purification by Isolera ${ }^{\circledR}$ with a basic alumina column cartridge (hexane) afforded $\mathbf{4 H}(31.2 \mathrm{mg}, 143 \mu \mathrm{mol}, 36 \%$ yield) as a colorless oil. A part of product underwent to decomposition (rearomatization) during purification. ${ }^{1} \mathrm{H}$ NMR $\left(400 \mathrm{MHz}, \mathrm{CDCl}_{3}\right) \delta 6.40(\mathrm{dd}, J=10.0,2.0 \mathrm{~Hz}, 1 \mathrm{H}), 6.12(\mathrm{dd}, J=10.0,2.0 \mathrm{~Hz}, 1 \mathrm{H}), 5.82-$ $5.70(\mathrm{~m}, 2 \mathrm{H}), 5.61(\mathrm{dd}, J=9.6,2.0 \mathrm{~Hz}, 1 \mathrm{H}), 5.30(\mathrm{~s}, 1 \mathrm{H}), 5.05-4.95(\mathrm{~m}, 2 \mathrm{H}), 2.15(\mathrm{~d}, J=7.2 \mathrm{~Hz}, 2 \mathrm{H})$, $1.10(\mathrm{~s}, 3 \mathrm{H}), 0.15(\mathrm{~s}, 9 \mathrm{H}) ;{ }^{13} \mathrm{C}$ NMR $\left(101 \mathrm{MHz}, \mathrm{CDCl}_{3}\right) \delta 144.6,138.5,137.1,134.6,130.0,127.6$, 125.2, 117.1, 46.8, 39.3, 27.7, 0.3; HRMS (DART) $m / z$ calcd for $\mathrm{C}_{14} \mathrm{H}_{23} \mathrm{Si}[\mathrm{M}+\mathrm{H}]^{+}: 219.1564$ found 219.1562 . 


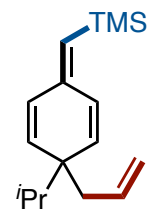

((4-Allyl-4-iso-propylcyclohexa-2,5-dien-1-ylidene)methyl)trimethylsilane (4I)

${ }^{1} \mathrm{H}$ NMR yield of $4 \mathrm{I}$ was $56 \%$ ( ${ }^{1} \mathrm{H}$ NMR peak at $5.29 \mathrm{ppm}$ (s, 1H) was used). Purification by Isolera $^{\circledR}$ with a basic alumina column cartridge (hexane) afforded $\mathbf{4 I}(9.8 \mathrm{mg}, 39.8 \mu \mathrm{mol}, 10 \%$ yield) as a colorless oil. A part of product underwent to decomposition (rearomatization) during purification. ${ }^{1} \mathrm{H}$ NMR (400 MHz, $\left.\mathrm{CDCl}_{3}\right) \delta 6.49(\mathrm{~d}, J=10.0 \mathrm{~Hz}, 1 \mathrm{H}), 6.22(\mathrm{~d}, J=10.0 \mathrm{~Hz}, 1 \mathrm{H}), 5.72-5.60(\mathrm{~m}, 2 \mathrm{H})$, $5.56(\mathrm{~d}, J=10.4 \mathrm{~Hz}, 1 \mathrm{H}), 5.29(\mathrm{~s}, 1 \mathrm{H}), 5.03-4.92(\mathrm{~m}, 2 \mathrm{H}), 2.22(\mathrm{~d}, J=7.2 \mathrm{~Hz}, 2 \mathrm{H}), 1.68-1.60(\mathrm{~m}$, $1 \mathrm{H}), 0.86(\mathrm{~d}, J=7.2 \mathrm{~Hz}, 6 \mathrm{H}), 0.15$ (s, 9H); ${ }^{13} \mathrm{C} \mathrm{NMR}\left(101 \mathrm{MHz}, \mathrm{CDCl}_{3}\right) \delta 145.2,136.0,135.0,134.6$, 132.0, 127.4, 127.3, 116.5, 46.2, 43.3, 36.1, 17.7, 0.3; HRMS (DART) $m / z$ calcd for $\mathrm{C}_{16} \mathrm{H}_{27} \mathrm{Si}[\mathrm{M}+$ $\mathrm{H}]^{+}: 247.1877$ found 247.1873 .

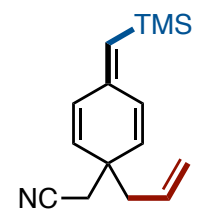

\section{2-(1-Allyl-4-((trimethylsilyl)methylene)cyclohexa-2,5-dien-1-yl)acetonitrile (4J)}

${ }^{1} \mathrm{H}$ NMR yield of $\mathbf{4 J}$ was $58 \%\left({ }^{1} \mathrm{H}\right.$ NMR peak at $5.51 \mathrm{ppm}(\mathrm{s}, 1 \mathrm{H})$ was used). Purification by Isolera ${ }^{\circledR}$ with a basic alumina column cartridge (hexane) afforded $\mathbf{4 J}$ (46.0 mg, $189 \mu \mathrm{mol}, 47 \%$ yield) as a colorless oil. A part of product underwent to decomposition (rearomatization) during purification. ${ }^{1} \mathrm{H}$ NMR $\left(400 \mathrm{MHz}, \mathrm{CDCl}_{3}\right) \delta 6.57(\mathrm{~d}, J=8.4 \mathrm{~Hz}, 1 \mathrm{H}), 6.27(\mathrm{~d}, J=8.4 \mathrm{~Hz}, 1 \mathrm{H}), 5.77-5.60(\mathrm{~m}, 3 \mathrm{H})$, $5.51(\mathrm{~s}, 1 \mathrm{H}), 5.16-5.09(\mathrm{~m}, 2 \mathrm{H}), 2.42(\mathrm{~s}, 2 \mathrm{H}), 2.34(\mathrm{~d}, J=8.0 \mathrm{~Hz}, 2 \mathrm{H}), 0.17(\mathrm{~s}, 9 \mathrm{H}) ;{ }^{13} \mathrm{C}$ NMR $(101$ $\left.\mathrm{MHz}, \mathrm{CDCl}_{3}\right) \delta 143.0,133.1,132.8,132.4,132.3,131.2,128.4,119.3,117.1,44.4,40.7,29.2,0.3$; HRMS (DART) $m / z$ calcd for $\mathrm{C}_{15} \mathrm{H}_{22} \mathrm{NSi}[\mathrm{M}+\mathrm{H}]^{+}: 244.1516$ found 244.1513 .

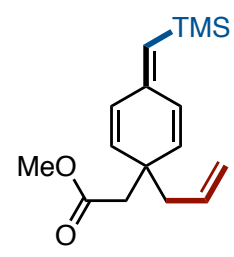

\section{Methyl 2-(1-allyl-4-((trimethylsilyl)methylene)cyclohexa-2,5-dien-1-yl)acetate (4K)}

${ }^{1} \mathrm{H}$ NMR yield of $4 \mathrm{~K}$ was $65 \%\left({ }^{1} \mathrm{H}\right.$ NMR peak at $5.37 \mathrm{ppm}(\mathrm{s}, 1 \mathrm{H})$ was used). Purification by Isolera ${ }^{\circledR}$ with a basic alumina column cartridge (hexane) afforded $\mathbf{4 K}(34.1 \mathrm{mg}, 123 \mu \mathrm{mol}, 31 \%$ yield) as a colorless oil. A part of product underwent to decomposition (rearomatization) during purification. ${ }^{1} \mathrm{H}$ NMR (400 MHz, $\left.\mathrm{CDCl}_{3}\right) \delta 6.48(\mathrm{dd}, J=10.0,1.6 \mathrm{~Hz}, 1 \mathrm{H}), 6.19(\mathrm{dd}, J=10.0,1.6 \mathrm{~Hz}, 1 \mathrm{H}), 5.79$ 
(dd, $J=10.0,1.6 \mathrm{~Hz}, 1 \mathrm{H}), 5.74-5.62(\mathrm{~m}, 2 \mathrm{H}), 5.37(\mathrm{~s}, 1 \mathrm{H}), 5.07-5.00(\mathrm{~m}, 2 \mathrm{H}), 3.61(\mathrm{~s}, 3 \mathrm{H}), 2.42(\mathrm{~s}$, 2H), $2.29(\mathrm{~d}, J=7.6 \mathrm{~Hz}, 2 \mathrm{H}), 0.15(\mathrm{~s}, 9 \mathrm{H}) ;{ }^{13} \mathrm{C} \mathrm{NMR}\left(101 \mathrm{MHz}, \mathrm{CDCl}_{3}\right) \delta 171.2,143.8,135.1,133.63$, 133.57, 131.5, 129.5, 126.7, 118.0, 51.4, 45.1, 45.0, 41.5, 0.3; HRMS (DART) $\mathrm{m} / \mathrm{z}$ calcd for $\mathrm{C}_{16} \mathrm{H}_{25} \mathrm{O}_{2} \mathrm{Si}[\mathrm{M}+\mathrm{H}]^{+}: 277.1618$ found 277.1616.

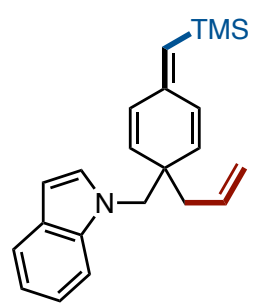

\section{1-((1-Allyl-4-((trimethylsilyl)methylene)cyclohexa-2,5-dien-1-yl)methyl)-1 $\mathrm{H}$-indole (4L)}

${ }^{1} \mathrm{H}$ NMR yield of $\mathbf{4} \mathbf{L}$ was $64 \%\left({ }^{1} \mathrm{H}\right.$ NMR peak at $5.37 \mathrm{ppm}(\mathrm{s}, 1 \mathrm{H})$ was used). Purification by Isolera $^{\circledR}$ with a basic alumina column cartridge (hexane) afforded $\mathbf{4 L}(73.8 \mathrm{mg}, 221 \mu \mathrm{mol}, 55 \%$ yield) as a colorless oil. A part of product underwent to decomposition (rearomatization) during purification. ${ }^{1} \mathrm{H}$ NMR $\left(400 \mathrm{MHz}, \mathrm{CDCl}_{3}\right) \delta 7.60(\mathrm{~d}, J=8.0 \mathrm{~Hz}, 1 \mathrm{H}), 7.34(\mathrm{~d}, J=8.8 \mathrm{~Hz}, 1 \mathrm{H}), 7.19(\mathrm{dd}, J=8.8,8.0$ $\mathrm{Hz}, 1 \mathrm{H}), 7.11-7.05(\mathrm{~m}, 2 \mathrm{H}), 6.52-6.46(\mathrm{~m}, 2 \mathrm{H}), 6.21$ (dd, $J=10.0,2.0 \mathrm{~Hz}, 1 \mathrm{H}), 5.70(\mathrm{dd}, J=10.0$, $2.0 \mathrm{~Hz}, 1 \mathrm{H}), 5.67-5.56(\mathrm{~m}, 2 \mathrm{H}), 5.37(\mathrm{~s}, 1 \mathrm{H}), 5.05-4.97(\mathrm{~m}, 2 \mathrm{H}), 4.10(\mathrm{~s}, 2 \mathrm{H}), 2.30(\mathrm{~d}, J=7.2 \mathrm{~Hz}, 2 \mathrm{H})$, 0.13 (s, 9H); ${ }^{13} \mathrm{C}$ NMR $\left(101 \mathrm{MHz}, \mathrm{CDCl}_{3}\right) \delta 143.8,137.1,134.1,133.7,132.7,132.5,130.1,129.3$, 128.2, 128.1, 121.3, 120.7, 119.2, 117.8, 110.2, 101.4, 55.1, 45.8, 43.2, 0.2; HRMS (DART) $\mathrm{m} / z$ calcd for $\mathrm{C}_{22} \mathrm{H}_{28} \mathrm{NSi}[\mathrm{M}+\mathrm{H}]^{+}: 334.1986$ found 334.1982.

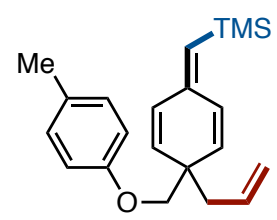

\section{((4-Allyl-4-((p-tolyloxy)methyl)cyclohexa-2,5-dien-1-ylidene)methyl)trimethylsilane (4M)}

The reaction was performed by using $\mathrm{Pd}(\mathrm{OAc})_{2}(4.5 \mathrm{mg}, 0.020 \mathrm{mmol}, 5 \mathrm{~mol} \%)$ instead of $\mathrm{Pd}_{2}(\mathrm{dba})_{3} \cdot \mathrm{CHCl}_{3}$. ${ }^{1} \mathrm{H}$ NMR yield of $\mathbf{4 M}$ was $67 \%\left({ }^{1} \mathrm{H}\right.$ NMR peak at $5.39 \mathrm{ppm}(\mathrm{s}, 1 \mathrm{H})$ was used). Purification by Isolera ${ }^{\circledR}$ with a basic alumina column cartridge (hexane) afforded $\mathbf{4 M}(41.8 \mathrm{mg}, 129$ $\mu \mathrm{mol}, 32 \%$ yield) as a colorless oil. A part of product underwent to decomposition (rearomatization) during purification. ${ }^{1} \mathrm{H}$ NMR $\left(400 \mathrm{MHz}, \mathrm{CDCl}_{3}\right) \delta 7.06(\mathrm{~d}, J=8.4 \mathrm{~Hz}, 2 \mathrm{H}), 6.78(\mathrm{~d}, J=8.4 \mathrm{~Hz}, 2 \mathrm{H})$, $6.54(\mathrm{~d}, J=10.0 \mathrm{~Hz}, 1 \mathrm{H}), 6.26$ (d, $J=10.0 \mathrm{~Hz}, 1 \mathrm{H}), 5.87$ (d, $J=10.0 \mathrm{~Hz}, 1 \mathrm{H}), 5.78-5.65$ (m, 2H), $5.40(\mathrm{~s}, 1 \mathrm{H}), 5.08-5.00(\mathrm{~m}, 2 \mathrm{H}), 3.76(\mathrm{~s}, 2 \mathrm{H}), 2.41(\mathrm{~d}, J=7.6 \mathrm{~Hz}, 2 \mathrm{H}), 2.27(\mathrm{~s}, 3 \mathrm{H}), 0.16(\mathrm{~s}, 9 \mathrm{H}) ;{ }^{13} \mathrm{C}$ NMR (101 MHz, $\left.\mathrm{CDCl}_{3}\right) \delta 157.0,144.3,134.0,133.9,132.4,130.0,129.8,129.3,127.6,117.7,114.5$, $73.9,43.7,41.7,20.5,0.3$ (one peak is missing due to overlapping); HRMS (DART) $\mathrm{m} / \mathrm{z}$ calcd for $\mathrm{C}_{21} \mathrm{H}_{29} \mathrm{OSi}[\mathrm{M}+\mathrm{H}]^{+}: 325.1982$ found 325.1979 . 


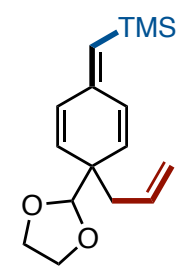

\section{((4-Allyl-4-(1,3-dioxolan-2-yl)cyclohexa-2,5-dien-1-ylidene)methyl)trimethylsilane (4N)}

${ }^{1} \mathrm{H}$ NMR yield of $4 \mathbf{N}$ was $42 \%\left({ }^{1} \mathrm{H}\right.$ NMR peak at $5.39 \mathrm{ppm}(\mathrm{s}, 1 \mathrm{H})$ was used). Purification by Isolera ${ }^{\circledR}$ with a basic alumina column cartridge (hexane) afforded $\mathbf{4 N}(29.8 \mathrm{mg}, 108 \mu \mathrm{mol}, 27 \%$ yield) as a colorless oil. A part of product underwent to decomposition (rearomatization) during purification. ${ }^{1} \mathrm{H}$ NMR $\left(400 \mathrm{MHz}, \mathrm{CDCl}_{3}\right) \delta 6.58(\mathrm{~d}, J=10.4 \mathrm{~Hz}, 1 \mathrm{H}), 6.30(\mathrm{~d}, J=10.4 \mathrm{~Hz}, 1 \mathrm{H}), 5.77(\mathrm{~d}, J=10.0$ $\mathrm{Hz}, 1 \mathrm{H}), 5.71-5.58(\mathrm{~m}, 2 \mathrm{H}), 5.39(\mathrm{~s}, 1 \mathrm{H}), 5.06-4.95(\mathrm{~m}, 2 \mathrm{H}), 4.73(\mathrm{~s}, 1 \mathrm{H}), 4.00-3.92(\mathrm{~m}, 2 \mathrm{H}), 3.90$ $3.82(\mathrm{~m}, 2 \mathrm{H}), 2.34(\mathrm{~d}, J=7.2 \mathrm{~Hz}, 2 \mathrm{H}), 0.15(\mathrm{~s}, 9 \mathrm{H}) ;{ }^{13} \mathrm{C} \mathrm{NMR}\left(101 \mathrm{MHz}, \mathrm{CDCl}_{3}\right) \delta 144.2,134.0$, 133.2, 132.0, 130.6, 129.7, 128.5, 117.2, 107.4, 65.40, 65.37, 47.5, 39.6, 0.3; HRMS (DART) $\mathrm{m} / \mathrm{z}$ calcd for $\mathrm{C}_{16} \mathrm{H}_{25} \mathrm{O} 2 \mathrm{Si}[\mathrm{M}+\mathrm{H}]^{+}: 277.1618$ found 277.1616.

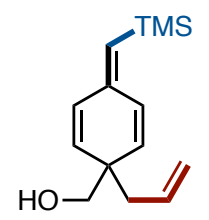

\section{(1-Allyl-4-((trimethylsilyl)methylene)cyclohexa-2,5-dien-1-yl)methanol (40)}

${ }^{1} \mathrm{H}$ NMR yield of $\mathbf{4 0}$ was $40 \%\left({ }^{1} \mathrm{H}\right.$ NMR peak at $5.43 \mathrm{ppm}(\mathrm{s}, 1 \mathrm{H})$ was used). Purification by Isolera $^{\circledR}$ with a basic alumina column cartridge (hexane) afforded $4 \mathbf{O}(27.7 \mathrm{mg}, 118 \mu \mathrm{mol}, 30 \%$ yield $)$ as a colorless oil. A part of product underwent to decomposition (rearomatization) during purification. ${ }^{1} \mathrm{H}$ NMR $\left(400 \mathrm{MHz}, \mathrm{CDCl}_{3}\right) \delta 6.63(\mathrm{~d}, J=10.0 \mathrm{~Hz}, 1 \mathrm{H}), 6.34(\mathrm{~d}, J=10.0 \mathrm{~Hz}, 1 \mathrm{H}), 5.74-5.64(\mathrm{~m}, 2 \mathrm{H})$, $5.60(\mathrm{~d}, J=10.0 \mathrm{~Hz}, 1 \mathrm{H}), 5.43(\mathrm{~s}, 1 \mathrm{H}), 5.06-5.01(\mathrm{~m}, 2 \mathrm{H}), 3.48(\mathrm{~s}, 2 \mathrm{H}), 2.19$ (d, $J=7.6 \mathrm{~Hz}, 2 \mathrm{H}), 1.40$ (brs, $1 \mathrm{H}), 0.16(\mathrm{~s}, 9 \mathrm{H}) ;{ }^{13} \mathrm{C}$ NMR $\left(101 \mathrm{MHz}, \mathrm{CDCl}_{3}\right) \delta 143.9,133.94,133.92,133.5,132.3,130.3$, 129.1, 117.6, 69.5, 46.1, 41.8, 0.3; HRMS (DART) $\mathrm{m} / z$ calcd for $\mathrm{C}_{14} \mathrm{H}_{23} \mathrm{OSi}[\mathrm{M}+\mathrm{H}]^{+}: 235.1513$ found 235.1511.

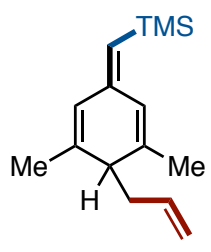

4P

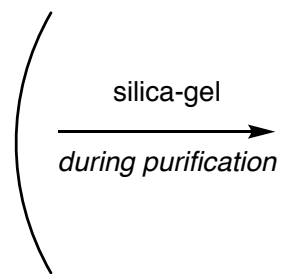

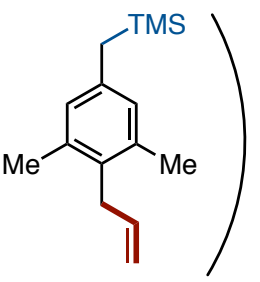

$4 \mathrm{P}$

((4-Allyl-3,5-dimethylcyclohexa-2,5-dien-1-ylidene)methyl)trimethylsilane (4P)

${ }^{1} \mathrm{H}$ NMR yield of $\mathbf{4 P}$ was $74 \%\left({ }^{1} \mathrm{H}\right.$ NMR peak at $5.10 \mathrm{ppm}(\mathrm{s}, 1 \mathrm{H})$ was used). 
During purification on silica-gel, $\mathbf{4 P}$ converted to (4-allyl-3,5-dimethylbenzyl)trimethylsilane (4P': $43.9 \mathrm{mg}, 189 \mu \mathrm{mol}, 47 \%$ yield) as a colorless oil.

(4-allyl-3,5-dimethylbenzyl)trimethylsilane (4P'): ${ }^{1} \mathrm{H}$ NMR (400 MHz, $\left.\mathrm{CDCl}_{3}\right) \delta 6.66(\mathrm{~s}, 2 \mathrm{H}), 5.94-$ $5.84(\mathrm{~m}, 1 \mathrm{H}), 4,98-4.95(\mathrm{~m}, 1 \mathrm{H}), 4.87-4.81(\mathrm{~m}, 1 \mathrm{H}), 3.34$ (d, $J=5.6 \mathrm{~Hz}, 2 \mathrm{H}), 2.24(\mathrm{~s}, 6 \mathrm{H}), 1.97$ (s, $2 \mathrm{H}),-0.01(\mathrm{~s}, 9 \mathrm{H}) ;{ }^{13} \mathrm{C} \mathrm{NMR}\left(101 \mathrm{MHz}, \mathrm{CDCl}_{3}\right) \delta 137.8,136.2,135.8,131.4,127.8,114.4,33.3,26.2$, 19.8, -1.8; HRMS (DART) $m / z$ calcd for $\mathrm{C}_{12} \mathrm{H}_{15} \mathrm{O}[\mathrm{M}+\mathrm{H}]^{+}: 233.1720$ found 233.1718.

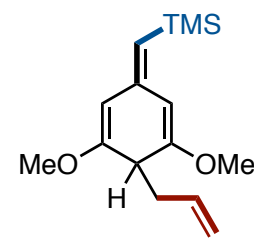

$4 Q$

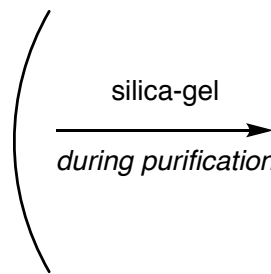

((4-Allyl-3,5-dimethoxycyclohexa-2,5-dien-1-ylidene)methyl)trimethylsilane (4Q)

${ }^{1} \mathrm{H}$ NMR yield of $\mathbf{4 Q}$ was $67 \%$ ( ${ }^{1} \mathrm{H}$ NMR peak at $5.39 \mathrm{ppm}$ (s, $\left.1 \mathrm{H}\right)$ was used).

During purification on silica-gel, 4Q converted to (4-allyl-3,5-dimethoxybenzyl)trimethylsilane (4Q': $42.6 \mathrm{mg}, 161 \mu \mathrm{mol}, 40 \%$ yield) as a colorless oil.

(4-Allyl-3,5-dimethoxybenzyl)trimethylsilane (4Q') ${ }^{1} \mathrm{H}$ NMR (400 MHz, $\left.\mathrm{CDCl}_{3}\right) \delta 6.19$ (s, 2H), 6.00-5.88 (m, 1H), 4.98-4.87 (m, 2H), $3.77(\mathrm{~s}, 6 \mathrm{H}), 3.35(\mathrm{~d}, J=6.0 \mathrm{~Hz}, 2 \mathrm{H}), 2.05(\mathrm{~s}, 2 \mathrm{H}), 0.01$ (s, 9H); ${ }^{13} \mathrm{C}$ NMR $\left(101 \mathrm{MHz}, \mathrm{CDCl}_{3}\right) \delta 157.9,139.8,137.4,113.6,111.9,103.9,55.8,27.6,27.0,-1.8$; HRMS (DART) $m / z$ calcd for $\mathrm{C}_{15} \mathrm{H}_{25} \mathrm{O}_{2} \mathrm{Si}[\mathrm{M}+\mathrm{H}]^{+}: 265.1618$ found 265.1614.

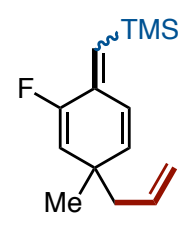

\section{((4-Allyl-2-fluoro-4-methylcyclohexa-2,5-dien-1-ylidene)methyl)trimethylsilane (4R)}

${ }^{1} \mathrm{H}$ NMR yield of $\mathbf{4 R}$ was $65 \%, \mathrm{dr}=78: 22\left({ }^{1} \mathrm{H}\right.$ NMR peaks at $5.81 \mathrm{ppm}(\mathrm{s}, 0.83 \mathrm{H})$ and $5.43 \mathrm{ppm}(\mathrm{s}$, $0.17 \mathrm{H}$ ) were used). Purification by Isolera ${ }^{\circledR}$ with a basic alumina column cartridge (hexane) afforded 4R (5.3 mg, $22.4 \mu \mathrm{mol}, 6 \%$ yield, $\mathrm{dr}=83: 17$ ) as a colorless oil and $45.4 \mathrm{mg}$ of mixture of $\mathbf{4 R}$ and starting material $\mathbf{1 R}$ in 87:13 ratio. A part of product underwent to decomposition (rearomatization) during purification. ${ }^{1} \mathrm{H}$ NMR $\left(400 \mathrm{MHz}, \mathrm{CDCl}_{3}\right) \delta 6.35(\mathrm{dd}, J=10.0,8.0 \mathrm{~Hz}, 0.83 \mathrm{H}), 6.09(\mathrm{dd}, J=$ $10.0,8.0 \mathrm{~Hz}, 0.16 \mathrm{H}), 5.81(\mathrm{~s}, 0.83 \mathrm{H}), 5.75-5.61(\mathrm{~m}, 1.88 \mathrm{H}), 5.54(\mathrm{~d}, J=10.0 \mathrm{~Hz}, 0.17 \mathrm{H}), 5.43$ (s, $0.17 \mathrm{H}), 5.30-5.24(\mathrm{~m}, 1 \mathrm{H}), 5.06-4.98(\mathrm{~m}, 2 \mathrm{H}), 2.18(\mathrm{~d}, J=7.6 \mathrm{~Hz}, 2 \mathrm{H}), 1.15(\mathrm{~s}, 3 \mathrm{H}), 0.18(\mathrm{~s}, 7.5 \mathrm{H})$, $0.13(\mathrm{~s}, 1.5 \mathrm{H}) ;{ }^{13} \mathrm{C}$ NMR $\left(101 \mathrm{MHz}, \mathrm{CDCl}_{3}\right) \delta 156.6\left(\mathrm{~d}, J_{\mathrm{C}-\mathrm{F}}=294 \mathrm{~Hz}\right), 155.6\left(\mathrm{~d}, J_{\mathrm{C}-\mathrm{F}}=254 \mathrm{~Hz}\right)$, $138.4\left(\mathrm{~d}, J_{\mathrm{C}-\mathrm{F}}=9.7 \mathrm{~Hz}\right), 138.1\left(\mathrm{~d}, J_{\mathrm{C}-\mathrm{F}}=22.2 \mathrm{~Hz}\right), 137.6,136.1,134.0,129.4\left(\mathrm{~d}, J_{\mathrm{C}-\mathrm{F}}=6.8 \mathrm{~Hz}\right), 127.5$, $124.5\left(\mathrm{~d}, J_{\mathrm{C}-\mathrm{F}}=3.8 \mathrm{~Hz}\right), 121.9,117.7,115.2\left(\mathrm{~d}, J_{\mathrm{C}-\mathrm{F}}=16.5 \mathrm{~Hz}\right), 113.3\left(\mathrm{~d}, J_{\mathrm{C}-\mathrm{F}}=16.5 \mathrm{~Hz}\right), 46.8,41.8$ 
$\left(\mathrm{d}, J_{\mathrm{C}-\mathrm{F}}=6.8 \mathrm{~Hz}\right), 41.1\left(\mathrm{~d}, J_{\mathrm{C}-\mathrm{F}}=7.8 \mathrm{~Hz}\right), 29.7,27.7,0.22,0.16$ (three peaks are overlapping); HRMS (DART) $m / z$ calcd for $\mathrm{C}_{14} \mathrm{H}_{22} \mathrm{FSi}[\mathrm{M}+\mathrm{H}]^{+}: 237.1469$ found 237.1466.

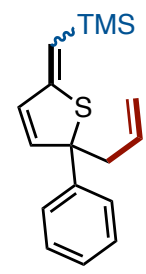

\section{((5-Allyl-5-phenylthiophen-2(5H)-ylidene)methyl)trimethylsilane (4S)}

${ }^{1} \mathrm{H}$ NMR yield of $4 \mathrm{~S}$ was $65 \%, \mathrm{dr}=57: 43\left({ }^{1} \mathrm{H}\right.$ NMR peaks at $5.56 \mathrm{ppm}(\mathrm{s}, 0.57 \mathrm{H})$ and $5.47 \mathrm{ppm}(\mathrm{s}$, $0.43 \mathrm{H}$ ) were used). Purification by Isolera ${ }^{\circledR}$ with a basic alumina column cartridge (hexane) afforded $4 \mathrm{~S}$ (45.3 $\mathrm{mg}, 158 \mu \mathrm{mol}, 40 \%$ yield) as a yellow oil. A part of product underwent to decomposition (rearomatization) during purification. ${ }^{1} \mathrm{H}$ NMR $\left(400 \mathrm{MHz}, \mathrm{CDCl}_{3}\right) \delta 7.41-7.19(\mathrm{~m}, 5 \mathrm{H}), 6.50(\mathrm{~d}, J=$ $6.4 \mathrm{~Hz}, 0.53 \mathrm{H}), 6.40(\mathrm{~d}, J=6.4 \mathrm{~Hz}, 0.48 \mathrm{H}), 6.31-6.22(\mathrm{~m}, 1 \mathrm{H}), 5.76-5.63(\mathrm{~m}, 1 \mathrm{H}), 5.56(\mathrm{~s}, 0.57 \mathrm{H})$, $5.47(\mathrm{~s}, 0.43 \mathrm{H}), 5.16-5.02(\mathrm{~m}, 2 \mathrm{H}), 2.97(\mathrm{~d}, J=7.6 \mathrm{~Hz}, 2 \mathrm{H}), 0.15(\mathrm{~s}, 5.5 \mathrm{H}), 0.14(\mathrm{~s}, 3.5 \mathrm{H}) ;{ }^{13} \mathrm{C} \mathrm{NMR}$ $\left(101 \mathrm{MHz}, \mathrm{CDCl}_{3}\right) \delta 158.5,156.7,144.1,144.0,143.5,140.2,134.5,133.7,130.0,128.8,128.4,127.0$, $126.4,126.3,125.3,118.4,118.3,116.6,115.6,71.3,69.2,46.8,46.7,0.4,-1.1$ (one peaks is missing due to overlapping); HRMS (DART) $m / z$ calcd for $\mathrm{C}_{17} \mathrm{H}_{23} \mathrm{SSi}[\mathrm{M}+\mathrm{H}]^{+}: 287.1284$ found 287.1280.

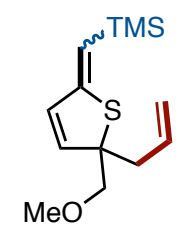

\section{((5-Allyl-5-(methoxymethyl)thiophen-2(5H)-ylidene)methyl)trimethylsilane (4T)}

${ }^{1} \mathrm{H}$ NMR yield of $\mathbf{4 T}$ was $65 \%, \mathrm{dr}=50: 50\left({ }^{1} \mathrm{H}\right.$ NMR peaks at $5.54 \mathrm{ppm}(\mathrm{s}, 0.5 \mathrm{H})$ and $5.42 \mathrm{ppm}(\mathrm{s}$, $0.5 \mathrm{H}$ ) were used). Purification by Isolera ${ }^{\circledR}$ with a basic alumina column cartridge (hexane) afforded $4 \mathrm{~T}$ (40.4 mg, $159 \mu \mathrm{mol}, 40 \%$ yield) as a colorless oil. A part of product underwent to decomposition (rearomatization) during purification. ${ }^{1} \mathrm{H}$ NMR $\left(400 \mathrm{MHz}, \mathrm{CDCl}_{3}\right) \delta 6.41(\mathrm{~d}, J=6.4 \mathrm{~Hz}, 0.5 \mathrm{H}), 6.19$ $6.15(\mathrm{~m}, 1 \mathrm{H}), 6.02(\mathrm{~d}, J=6.4 \mathrm{~Hz}, 0.5 \mathrm{H}), 5.84-5.74(\mathrm{~m}, 1 \mathrm{H}), 5.54(\mathrm{~s}, 0.5 \mathrm{H}), 5.42(\mathrm{~s}, 0.5 \mathrm{H}), 5.16-5.09$ (m, 2H), 3.52-3.46 (m, 2H), $3.371(\mathrm{~s}, 1.5 \mathrm{H}), 3.367(\mathrm{~s}, 1.5 \mathrm{H}), 2.67-2.53(\mathrm{~m}, 2 \mathrm{H}), 0.15(\mathrm{~s}, 4.5 \mathrm{H}), 0.13$ $(\mathrm{s}, 4.5 \mathrm{H}) ;{ }^{13} \mathrm{C} \mathrm{NMR}\left(101 \mathrm{MHz}, \mathrm{CDCl}_{3}\right) \delta 158.2,156.5,142.4,139.1,135.3,133.8,130.8,118.5,118.4$, $116.4,115.3,78.4,78.2,69.7,67.4,59.32,59.27,41.5,41.4,0.4-1.1$ (one peak is missing due to overlapping); HRMS (DART) $m / z$ calcd for $\mathrm{C}_{13} \mathrm{H}_{23} \mathrm{OSSi}[\mathrm{M}+\mathrm{H}]^{+}: 255.1233$ found 255.1228 . 
<smiles>C=CCC1(c2ccccn2)C=CC(=CC)S1</smiles>

\section{2-(2-Allyl-5-((trimethylsilyl)methylene)-2,5-dihydrothiophen-2-yl)pyridine (4U)}

${ }^{1} \mathrm{H}$ NMR yield of $\mathbf{4} \mathbf{U}$ was $57 \%, \mathrm{dr}=58: 42\left({ }^{1} \mathrm{H}\right.$ NMR peaks at $5.58 \mathrm{ppm}(\mathrm{s}, 0.42 \mathrm{H})$ and $5.48 \mathrm{ppm}(\mathrm{s}$, $0.58 \mathrm{H}$ ) were used). Purification by Isolera ${ }^{\circledR}$ with a basic alumina column cartridge (hexane) afforded 4U as a colorless oil $(57.0 \mathrm{mg}, 198 \mu \mathrm{mol}, 50 \%$ yield). A part of product underwent to decomposition (rearomatization) during purification. ${ }^{1} \mathrm{H} \mathrm{NMR}\left(400 \mathrm{MHz}, \mathrm{CDCl}_{3}\right) \delta 8.58-8.56(\mathrm{~m}, 1 \mathrm{H}), 7.65-7.61(\mathrm{~m}$, 1H), 7.39-7.36 (m, 1H), 7.15-7.12 (m, 1H), $6.55(\mathrm{~d}, J=6.4 \mathrm{~Hz}, 0.42 \mathrm{H}), 6.52(\mathrm{~d}, J=6.4 \mathrm{~Hz}, 0.42 \mathrm{H})$, $6.37(\mathrm{~d}, J=6.0 \mathrm{~Hz}, 0.58 \mathrm{H}), 6.29(\mathrm{~d}, J=6.0 \mathrm{~Hz}, 0.58 \mathrm{H}), 5.73-5.62(\mathrm{~m}, 1 \mathrm{H}), 5.58(\mathrm{~s}, 0.42 \mathrm{H}), 5.48$ (s, $0.58 \mathrm{H}), 5.09-5.01(\mathrm{~m}, 2 \mathrm{H}), 3.07(\mathrm{~d}, J=6.8 \mathrm{~Hz}, 2 \mathrm{H}), 0.15(\mathrm{~s}, 9 \mathrm{H}) ;{ }^{13} \mathrm{C} \mathrm{NMR}\left(101 \mathrm{MHz}, \mathrm{CDCl}_{3}\right) \delta$ 162.6, 162.5, 158.2, 156.5, 148.94, 148.91, 142.8, 139.4, 136.6, 135.2, 133.7, 130.5, 121.8, 121.5, $121.4,118.4,118.3,116.9,116.0,73.4,71.3,46.10,46.06,0.4,-1.1$ (three peaks are missing due to overlapping.); HRMS (ESI) $m / z$ calcd for $\mathrm{C}_{16} \mathrm{H}_{22} \mathrm{NSSi}[\mathrm{M}+\mathrm{H}]^{+}: 288.1237$ found 288.1230 .

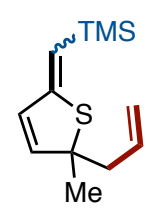

\section{((5-Allyl-5-methylthiophen-2(5H)-ylidene)methyl)trimethylsilane (4V)}

${ }^{1} \mathrm{H}$ NMR yield of $\mathbf{4 V}$ was $67 \%, \mathrm{dr}=53: 47\left({ }^{1} \mathrm{H}\right.$ NMR peaks at $5.51 \mathrm{ppm}(\mathrm{s}, 0.53 \mathrm{H})$ and $5.38 \mathrm{ppm}(\mathrm{s}$, $0.47 \mathrm{H}$ ) were used). Purification by Isolera ${ }^{\circledR}$ with a basic alumina column cartridge (hexane) afforded $4 \mathrm{~V}$ as a colorless oil $(37.5 \mathrm{mg}, 167 \mu \mathrm{mol}, 42 \%$ yield). A part of product underwent to decomposition (rearomatization) during purification. ${ }^{1} \mathrm{H} \mathrm{NMR}\left(400 \mathrm{MHz}^{\mathrm{CDCl}} 3\right) \delta 6.32(\mathrm{~d}, J=6.0 \mathrm{~Hz}, 0.53 \mathrm{H}), 6.09$ $6.02(\mathrm{~m}, 1 \mathrm{H}), 5.90(\mathrm{~d}, J=6.0 \mathrm{~Hz}, 0.47 \mathrm{H}), 5.88-5.75(\mathrm{~m}, 1 \mathrm{H}), 5.51(\mathrm{~s}, 0.53 \mathrm{H}), 5.38(\mathrm{~s}, 0.47 \mathrm{H}), 5.15-$ $5.03(\mathrm{~m}, 2 \mathrm{H}), 2.48(\mathrm{~d}, J=6.8 \mathrm{~Hz}, 2 \mathrm{H}), 1.52(\mathrm{~s}, 1.5 \mathrm{H}), 1.51(\mathrm{~s}, 1.5 \mathrm{H}), 0.15(\mathrm{~s}, 4.1 \mathrm{H}), 0.14(\mathrm{~s}, 4.9 \mathrm{H}) ;{ }^{13} \mathrm{C}$ NMR (101 MHz, $\left.\mathrm{CDCl}_{3}\right) \delta 159.1,157.4,146.2,143.0,134.2,134.1,133.3,128.9,118.3,118.2,115.6$, 114.5, 66.0, 63.7, 47.3, 47.1, 28.4, 28.2, 0.4, -1.1; HRMS (DART) $\mathrm{m} / \mathrm{z}$ calcd for $\mathrm{C}_{12} \mathrm{H}_{21} \mathrm{SSi}[\mathrm{M}+\mathrm{H}]^{+}$: 225.1128 found 225.1123 .

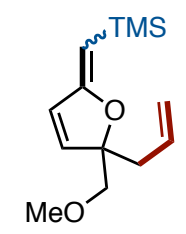

((5-Allyl-5-(methoxymethyl)furan-2(5H)-ylidene)methyl)trimethylsilane (4W) 
${ }^{1} \mathrm{H}$ NMR yield of $\mathbf{4 W}$ was $56 \%$, ( ${ }^{1} \mathrm{H}$ NMR peak at $4.10 \mathrm{ppm}(\mathrm{s}, 1 \mathrm{H})$ was used). Purification by Isolera ${ }^{\circledR}$ with a basic alumina column cartridge (hexane) afforded $\mathbf{4 W}(20.2 \mathrm{mg}, 84.7 \mu \mathrm{mol}, 21 \%$ yield) as a colorless oil. A part of product underwent to decomposition (rearomatization) during purification. ${ }^{1} \mathrm{H}$ NMR $\left(400 \mathrm{MHz}, \mathrm{CDCl}_{3}\right) \delta 6.21(\mathrm{~d}, J=6.0 \mathrm{~Hz}, 1 \mathrm{H}), 6.10(\mathrm{~d}, J=6.0 \mathrm{~Hz}, 1 \mathrm{H}), 5.77-$ $5.67(\mathrm{~m}, 1 \mathrm{H}), 5.12-5.06(\mathrm{~m}, 2 \mathrm{H}), 4.10(\mathrm{~s}, 1 \mathrm{H}), 3.49$ (d, $J=10.0 \mathrm{~Hz}, 1 \mathrm{H}), 3.39$ (d, $J=10.0 \mathrm{~Hz}, 1 \mathrm{H})$, 3.38 (s, 3H), 2.51-2.39 (m, 2H), 0.11 (s, 9H); $\left.{ }^{13} \mathrm{C} \mathrm{NMR} \mathrm{(101} \mathrm{MHz,} \mathrm{CDCl}_{3}\right) \delta 169.7,137.8,132.4$, 128.1, 118.4, 93.9, 90.4, 76.3, 59.8, 39.9, -0.3; HRMS (DART) $\mathrm{m} / z$ calcd for $\mathrm{C}_{13} \mathrm{H}_{23} \mathrm{O}_{2} \mathrm{Si}[\mathrm{M}+\mathrm{H}]^{+}$: 239.1462 found 239.1458.

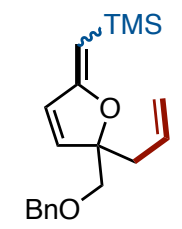

\section{((5-Allyl-5-((benzyloxy)methyl)furan-2(5H)-ylidene)methyl)trimethylsilane (4X)}

${ }^{1} \mathrm{H}$ NMR yield of $\mathbf{4 X}$ was $45 \%$, $\left({ }^{1} \mathrm{H}\right.$ NMR peak at $4.11 \mathrm{ppm}(\mathrm{d}, J=1.2 \mathrm{~Hz}, 1 \mathrm{H})$ was used). Purification by Isolera ${ }^{\circledR}$ with a basic alumina column cartridge (hexane) afforded $\mathbf{4 X}$ (30.1 mg, 95.7 mol, 24\% yield) as a colorless oil. A part of product underwent to decomposition (rearomatization) during purification. ${ }^{1} \mathrm{H}$ NMR $\left(400 \mathrm{MHz}, \mathrm{CDCl}_{3}\right) \delta 7.37-7.27(\mathrm{~m}, 5 \mathrm{H}), 6.24(\mathrm{dd}, J=6.0,1.2 \mathrm{~Hz}, 1 \mathrm{H})$, $6.10(\mathrm{~d}, J=6.0 \mathrm{~Hz}, 1 \mathrm{H}), 5.77-5.67(\mathrm{~m}, 1 \mathrm{H}), 5.11-5.06(\mathrm{~m}, 2 \mathrm{H}), 4.57$ (d, $J=2.8 \mathrm{~Hz}, 2 \mathrm{H}), 4.11(\mathrm{~d}, J=$ $1.2 \mathrm{~Hz}, 1 \mathrm{H}), 3.57(\mathrm{~d}, J=10.0 \mathrm{~Hz}, 1 \mathrm{H}), 3.49$ (d, $J=10.0 \mathrm{~Hz}, 1 \mathrm{H}), 2.55-2.43(\mathrm{~m}, 2 \mathrm{H}), 0.12(\mathrm{~s}, 9 \mathrm{H}) ;{ }^{13} \mathrm{C}$ NMR $\left(101 \mathrm{MHz}, \mathrm{CDCl}_{3}\right) \delta 169.7,138.3,138.0,132.4,128.4,128.2,127.6,127.5,118.4,94.1,90.5$, 73.8, 73.7, 40.0, -0.2; HRMS (ESI) $m / z$ calcd for $\mathrm{C}_{19} \mathrm{H}_{27} \mathrm{O}_{2} \mathrm{Si}[\mathrm{M}+\mathrm{H}]^{+}: 315.1775$ found 315.1769 .

\section{One-Pot Dearomative Reaction of 2-Phenylthiophene (6)}

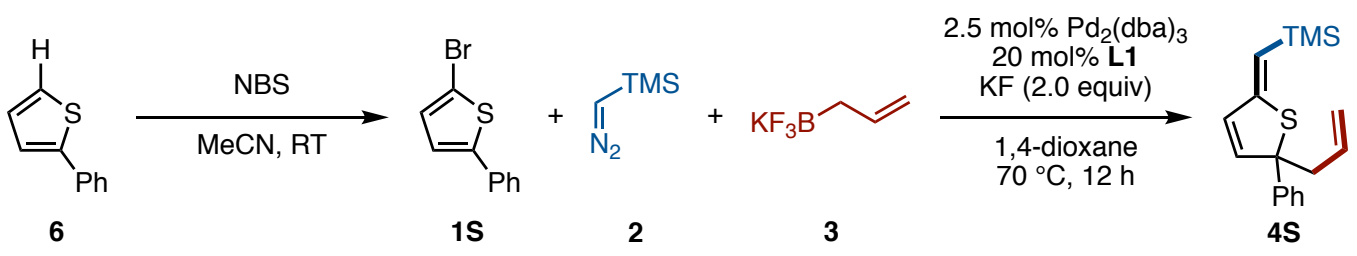

To an 8-mL glass vessel equipped with a screw cap containing a magnetic stirring bar and 2-phenylthiophene (32.1 mg, $0.20 \mathrm{mmol}, 1.0$ equiv) were added $N$-bromosuccinimide (NBS: 1.1 equiv) and $\mathrm{MeCN}(0.40 \mathrm{~mL})$. After stirring the mixture for $45 \mathrm{~min}$ with monitoring reaction progress by TLC, the mixture was concentrated in vacuo to remove $\mathrm{MeCN}$.

To the same tube containing obtained crude product $1 \mathrm{~S}$ were added heat-gun-dried potassium fluoride (23.2 mg, $0.40 \mathrm{mmol}, 2.0$ equiv), $\mathrm{Pd}_{2}(\mathrm{dba})_{3} \cdot \mathrm{CHCl}_{3}(5.18 \mathrm{mg}, 5.0 \mu \mathrm{mol}, 2.5 \mathrm{~mol} \%$ ), 4-(dimethylamino)phenyldiphenylphosphine (L1: $12.2 \mathrm{mg}, 0.040 \mathrm{mmol}, 20 \mathrm{~mol} \%$ ), potassium allyltrifluoroborate (3: $38.5 \mathrm{mg}, 0.26 \mathrm{mmol}, 1.3$ equiv). The vessel was placed under vacuum and 
refilled $\mathrm{N}_{2}$ gas three times, and then added 1,4-dioxane $(1.0 \mathrm{~mL})$. The mixture was stirred for several minutes until the color of the solution turned to yellow. A solution of (trimethylsilyl)diazomethane (2, 2.0 $\mathrm{M}$ in $\mathrm{Et}_{2} \mathrm{O}: 0.13 \mathrm{~mL}, 0.26 \mathrm{mmol}, 1.3$ equiv) was then added slowly. The vessel was sealed with a screw cap and then heated at $70{ }^{\circ} \mathrm{C}$ for $12 \mathrm{~h}$ with stirring. After cooling the reaction mixture to room temperature, the mixture was passed through a pad of alumina (hexane) and then concentrated in vacuo. The yield of 4 was determined by ${ }^{1} \mathrm{H}$ NMR analysis using $\mathrm{CH}_{2} \mathrm{Br}_{2}$ as an internal standard. ${ }^{1} \mathrm{H}$ NMR yield of $4 \mathrm{~S}$ was $55 \%\left({ }^{1} \mathrm{H}\right.$ NMR peaks at $5.56 \mathrm{ppm}(\mathrm{s}, 0.57 \mathrm{H})$ and $5.47 \mathrm{ppm}(\mathrm{s}, 0.43 \mathrm{H})$ were used). 


\section{Application to A Pharmaceutical Compound}

\section{Synthesis of Brominated Ticlopidine (7)}
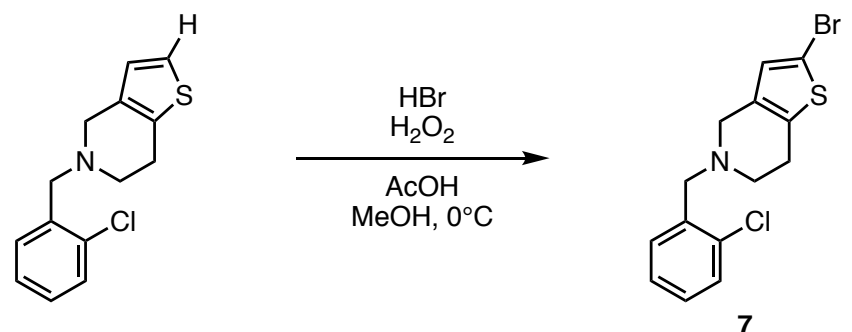

To an $8-\mathrm{mL}$ glass vessel containing ticlopidine (131.9 $\mathrm{mg}, 0.50 \mathrm{mmol}, 1.0$ equiv), $\mathrm{HBr}(47 \%$ in water; $374 \mu \mathrm{L}, 40.1 \mathrm{mmol}, 80.1$ equiv), $\mathrm{AcOH}(0.50 \mathrm{~mL})$, and $\mathrm{MeOH}(0.50 \mathrm{~mL})$, was added $\mathrm{H}_{2} \mathrm{O}_{2}$ (30\% in water; $165 \mu \mathrm{L}, 1.65 \mathrm{mmol}, 3.29$ equiv) in $\mathrm{MeOH}(0.50 \mathrm{~mL})$ slowly at $0{ }^{\circ} \mathrm{C}$. After stirring the mixture at $0{ }^{\circ} \mathrm{C}$ for $1.5 \mathrm{~h}$ with monitoring reaction progress by TLC, the reaction was quenched with $\mathrm{NaHCO}_{3}$ aq. and $\mathrm{Na}_{2} \mathrm{~S}_{2} \mathrm{O}_{3}$ aq. at $0{ }^{\circ} \mathrm{C}$. The mixture was extracted three times with $\mathrm{CH}_{2} \mathrm{Cl}_{2}$. The combined organic layer was dried over $\mathrm{Na}_{2} \mathrm{SO}_{4}$, filtrated, and concentrated in vacuo. The residue was purified by Isolera ${ }^{\circledR}$ (hexane/EtOAc $=19: 1$ to $\left.4: 1\right)$ to afford 7 (95.4 $\mathrm{mg}, 278 \mu \mathrm{mol}, 56 \%$ yield) as a colorless oil. ${ }^{1} \mathrm{H}$ NMR $\left(400 \mathrm{MHz}, \mathrm{CDCl}_{3}\right) \delta 7.51(\mathrm{~d}, J=7.2 \mathrm{~Hz}, 1 \mathrm{H}), 7.37(\mathrm{~d}, J=8.0 \mathrm{~Hz}, 1 \mathrm{H}), 7.27-$ $7.19(\mathrm{~m}, 2 \mathrm{H}), 6.67$ (s, 1H), 3.80 (s, 2H), 3.55 (s, 2H), 2.86-2.80 (m, 4H); $\left.{ }^{13} \mathrm{C} \mathrm{NMR} \mathrm{(101} \mathrm{MHz,} \mathrm{CDCl}_{3}\right)$ $\delta 135.8,135.1,134.4,134.2,130.6,129.5,128.3,128.0,126.7,109.3,58.3,52.5,50.4,25.4$. HRMS (DART) $m / z$ calcd for $\mathrm{C}_{14} \mathrm{H}_{14} \mathrm{NBrClS}[\mathrm{M}+\mathrm{H}]^{+}: 341.9713$ found 341.9705 .

\section{Pd-Catalyzed Dearomative Three-Component Reaction of 7}

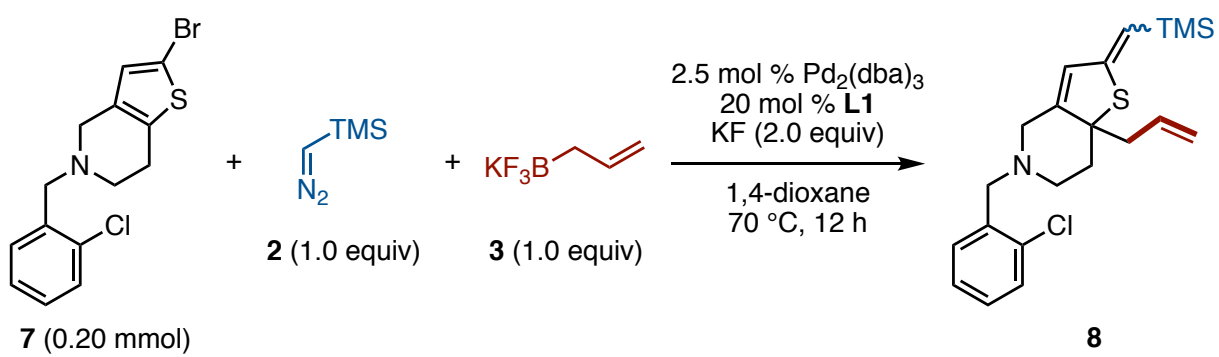

Using the general procedure: ${ }^{1} \mathrm{H}$ NMR yield of 8 was $57 \%, \mathrm{dr}=44: 56\left({ }^{1} \mathrm{H}\right.$ NMR peaks at $5.46 \mathrm{ppm}$ $(\mathrm{s}, 0.5 \mathrm{H})$ and $5.33 \mathrm{ppm}(\mathrm{s}, 0.5 \mathrm{H})$ were used). Purification by Isolera ${ }^{\circledR}$ with a basic alumina column cartridge (hexane/EtOAc $=99: 1$ to 3:2) to afford dearomatized ticlopidine 8 (37.1 mg, $95.1 \mu \mathrm{mol}$, $48 \%$ yield, $\mathrm{dr}=50: 50)$ as a colorless oil. A part of product underwent to decomposition (rearomatization) during purification. ${ }^{1} \mathrm{H}$ NMR $\left(400 \mathrm{MHz}, \mathrm{CDCl}_{3}\right) \delta$ 7.48-7.44 (m, 1H), 7.38-7.34 (m, 1H), 7.27-7.19 (m, 2H), $6.14(\mathrm{~s}, 0.5 \mathrm{H}), 5.93(\mathrm{~s}, 0.5 \mathrm{H}), 5.84-5.74(\mathrm{~m}, 1 \mathrm{H}), 5.46(\mathrm{~s}, 0.5 \mathrm{H}), 5.33(\mathrm{~s}$, $0.5 \mathrm{H}), 5.15-5.10(\mathrm{~m}, 2 \mathrm{H}), 3.80-3.67(\mathrm{~m}, 2 \mathrm{H}), 3.50-3.43(\mathrm{~m}, 1 \mathrm{H}), 3.08-2.97(\mathrm{~m}, 1 \mathrm{H}), 2.85-2.81(\mathrm{~m}$, $1 \mathrm{H}), 2.73-2.53(\mathrm{~m}, 3 \mathrm{H}), 2.30-2.19(\mathrm{~m}, 1 \mathrm{H}), 2.12-2.08(\mathrm{~m}, 1 \mathrm{H}), 0.14(\mathrm{~s}, 4.5 \mathrm{H}), 0.13(\mathrm{~s}, 4.5 \mathrm{H}) ;{ }^{13} \mathrm{C}$ NMR $\left(101 \mathrm{MHz}, \mathrm{CDCl}_{3}\right) \delta 157.5,155.6,152.1,148.7,135.8,135.6,134.2,134.1,133.7,133.6,130.8$, 
130.6, 130.0, 129.5, 129.4, 128.3, 128.2, 126.73, 126.68, 125.7, 118.5, 118.3, 114.2, 113.3, 64.8, 62.7, 58.10, 58.06, 51.9, 51.6, 49.5, 49.3, 41.0, 40.9, 38.7, 38.2, 0.4, -1.1; HRMS (DART) $\mathrm{m} / z$ calcd for $\mathrm{C}_{21} \mathrm{H}_{29} \mathrm{NClSSi}[\mathrm{M}+\mathrm{H}]^{+}: 390.1473$ found 390.1467.

\section{Derivatization of Products}

\section{Diimide Reduction of 4M}
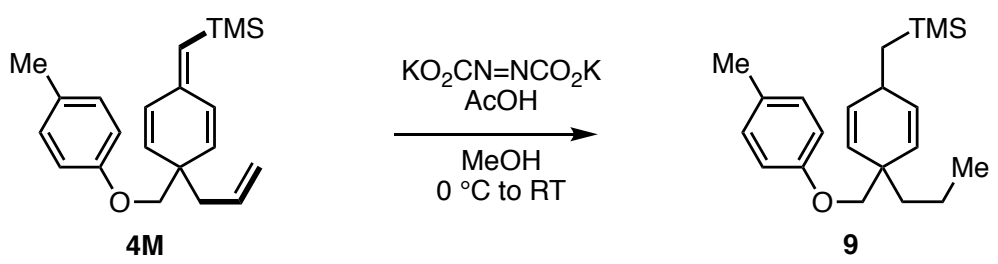

crude

mixture

To a crude mixture of $\mathbf{4 M}$ (NMR yield was $67 \%, 0.40 \mathrm{mmol}$ scale) in a $20 \mathrm{~mL}$ vial with a magnetic stirring bar were added potassium diazocarboxylate $(582 \mathrm{mg}, 3.0 \mathrm{mmol}, 7.5$ equiv based on the amount of $1 \mathrm{M})$ and $\mathrm{MeOH}(4.0 \mathrm{~mL})$. The mixture was cooled at $0{ }^{\circ} \mathrm{C}$, and then $\mathrm{AcOH}(337 \mu \mathrm{L}, 5.9$ mmol, 24.3 equiv) was slowly added. After stirring $6 \mathrm{~h}$ at room temperature, potassium diazocarboxylate (582 mg, $3.0 \mathrm{mmol}, 7.5$ equiv) and $\mathrm{AcOH}$ were again added in this order at $0{ }^{\circ} \mathrm{C}$. The mixture was further stirred at room temperature until the color of suspension turned to white from yellow. The mixture was slowly added $\mathrm{NaHCO}_{3}$ aq. and extracted three times with EtOAc. Combined organic layer was dried over $\mathrm{Na}_{2} \mathrm{SO}_{4}$, filtrated, and concentrated in vacuo. The mixture was purified by Isolera $^{\circledR}$ with basic alumina column cartridge (hexane) to afford trimethyl((4-propyl-4-((p-tolyloxy)methyl)cyclohexa-2,5-dien-1-yl)methyl)silane 9 (72.5 $\mathrm{mg}$, as a mixture of diastereoisomers in a 57:43 ratio, 55\% yield in 2 steps) as a colorless oil. ${ }^{1} \mathrm{H}$ NMR (400 $\left.\mathrm{MHz}, \mathrm{CDCl}_{3}\right) \delta 7.04(\mathrm{~d}, J=8.4 \mathrm{~Hz}, 2 \mathrm{H}), 6.77$ (d, $\left.J=8.4 \mathrm{~Hz}, 2 \mathrm{H}\right), 5.82-5.74(\mathrm{~m}, 2 \mathrm{H}), 5.50-5.45(\mathrm{~m}$, $2 \mathrm{H}), 3.66(\mathrm{~s}, 2 \mathrm{H}), 2.83-2.74(\mathrm{~m}, 1 \mathrm{H}), 2.27(\mathrm{~s}, 3 \mathrm{H}), 1.50-1.44(\mathrm{~m}, 2 \mathrm{H}), 1.28-1.18(\mathrm{~m}, 2 \mathrm{H}), 0.93-0.85$ $(\mathrm{m}, 3 \mathrm{H}), 0.73(\mathrm{~d}, J=7.6 \mathrm{~Hz}, 0.88 \mathrm{H}), 0.69$ (d, $J=7.6 \mathrm{~Hz}, 1.12 \mathrm{H}), 0.06(\mathrm{~s}, 5.1 \mathrm{H}), 0.05(\mathrm{~s}, 3.9 \mathrm{H}) ;{ }^{13} \mathrm{C}$ NMR $\left(101 \mathrm{MHz}, \mathrm{CDCl}_{3}\right) \delta 157.3,132.8,132.7,129.7,128.3,128.2,114.6,114.4,76.1,75.9,41.2$, $39.7,39.1,32.6,32.3,25.4,25.2,20.5,18.2,17.9,14.7,-0.46,-0.54$ (several peaks are overlapping). HRMS (DART) $m / z$ calcd for $\mathrm{C}_{21} \mathrm{H}_{36} \mathrm{NOSi}\left[\mathrm{M}+\mathrm{NH}_{4}\right]^{+}: 346.2561$ found 346.2556.

\section{Oxidation of 9}

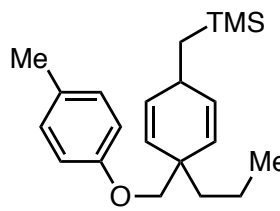

9

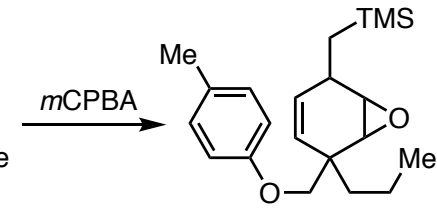

10

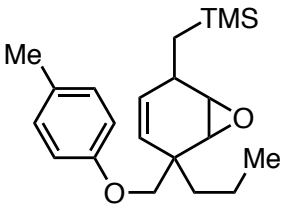

10-dr 
To a solution of $9\left(58.3 \mathrm{mg}, 0.18 \mu \mathrm{mol}, 1.0\right.$ equiv) in $\mathrm{CH}_{2} \mathrm{Cl}_{2}(2.0 \mathrm{~mL})$ was added a solution meta-chloroperbenzoic acid (77\% purity, $52.7 \mathrm{mg}, 0.27 \mathrm{mmol}, 1.3$ equiv) at $0{ }^{\circ} \mathrm{C}$. After stirring the solution at $0{ }^{\circ} \mathrm{C}$ for 3 hours, $\mathrm{Na}_{2} \mathrm{~S}_{2} \mathrm{O}_{3}$ aq. was added to quench the reaction. The mixture was extracted three times with $\mathrm{CH}_{2} \mathrm{Cl}_{2}$, dried over $\mathrm{Na}_{2} \mathrm{SO}_{4}$, filtrated, and then concentrated in vacuo. Purification by PTLC (hexane/EtOAc $=100: 1)$ afforded $10(17.2 \mathrm{mg}, 50 \mu \mathrm{mol}, 28 \%$ yield $)$ as a colorless oil and 10-dr (13.9 mg, $40 \mu \mathrm{mol}, 23 \%$ yield) as a colorless oil.

10: ${ }^{1} \mathrm{H}$ NMR (400 MHz, $\left.\mathrm{CDCl}_{3}\right) \delta 7.07(\mathrm{~d}, J=8.4 \mathrm{~Hz}, 2 \mathrm{H}), 6.83(\mathrm{~d}, J=8.4 \mathrm{~Hz}, 2 \mathrm{H}), 5.57$ (ddd, $J=$ $10.4,4.4,1.6 \mathrm{~Hz}, 1 \mathrm{H}), 5.19$ (d, $J=10.4 \mathrm{~Hz}, 1 \mathrm{H}), 3.91$ (d, $J=8.8 \mathrm{~Hz}, 1 \mathrm{H}), 3.75$ (d, $J=8.8 \mathrm{~Hz}, 1 \mathrm{H})$, 320-3.19 (m, 1H), 3.12-3.10 (m, 1H), 2.70-2.64 (m, 1H), 2.28 (s, 3H), 1.72-1.66 (m, 2H), 1.35-1.27 (m, 2H), 0.94 (t, $J=7.2 \mathrm{~Hz}, 3 \mathrm{H}), 0.80$ (dd, $J=14.4,6.0 \mathrm{~Hz}, 1 \mathrm{H}), 0.64$ (dd, $J=14.4,10.0 \mathrm{~Hz}, 1 \mathrm{H})$, 0.08 (s, 9H); ${ }^{13} \mathrm{C}$ NMR $\left(100 \mathrm{MHz}, \mathrm{CDCl}_{3}\right) \delta 157.1,129.82,129.78,129.3,124.6,114.5,72.1,57.2$, $57.8,40.1,37.3,31.7,20.7,20.5,17.8,14.8,-0.8$; HRMS (DART) $m / z$ calcd for $\mathrm{C}_{21} \mathrm{H}_{33} \mathrm{O}_{2} \mathrm{Si}^{+}[\mathrm{M}+$ $\mathrm{H}]^{+}: 345.2244$ found 345.2236 .

10-dr: ${ }^{1} \mathrm{H}$ NMR (400 MHz, $\left.\mathrm{CDCl}_{3}\right) \delta 7.06(\mathrm{~d}, J=8.4 \mathrm{~Hz}, 2 \mathrm{H}), 6.82(\mathrm{~d}, J=8.4 \mathrm{~Hz}, 2 \mathrm{H}), 5.38(\mathrm{~d}, J=$ $10.8 \mathrm{~Hz}, 1 \mathrm{H}), 5.19$ (d, $J=10.8 \mathrm{~Hz}, 1 \mathrm{H}), 3.91$ (d, $J=8.8 \mathrm{~Hz}, 1 \mathrm{H}), 3.74$ (d, $J=8.8 \mathrm{~Hz}, 1 \mathrm{H}), 3.27-3.24$ (m, 2H), 2.57-2.53 (m, 1H), $2.27(\mathrm{~s}, 3 \mathrm{H}), 1.77-1.71(\mathrm{~m}, 1 \mathrm{H}), 1.65-1.57(\mathrm{~m}, 1 \mathrm{H}), 1.35-1.25(\mathrm{~m}, 2 \mathrm{H})$, $0.93(\mathrm{t}, J=7.2 \mathrm{~Hz}, 3 \mathrm{H}), 0.86(\mathrm{dd}, J=14.8,8.0 \mathrm{~Hz}, 1 \mathrm{H}), 0.76(\mathrm{dd}, J=14.8,7.2 \mathrm{~Hz}, 1 \mathrm{H}), 0.09$ (s, 9H): ${ }^{13} \mathrm{C}$ NMR $\left(101 \mathrm{MHz}, \mathrm{CDCl}_{3}\right) \delta 157.1,129.9,129.8,125.2,114.5,72.3,57.8,56.9,40.1,37.3,31.7$, 20.7, 20.5, 17.2, 14.7, -0.7 (one carbon is missing due to overlapping). HRMS (DART) $\mathrm{m} / z$ calcd for $\mathrm{C}_{21} \mathrm{H}_{36} \mathrm{NO}_{2} \mathrm{Si}\left[\mathrm{M}+\mathrm{NH}_{4}\right]^{+}: 362.2510$ found 362.2499.

\section{Structure Determination of 10 and 10-dr}

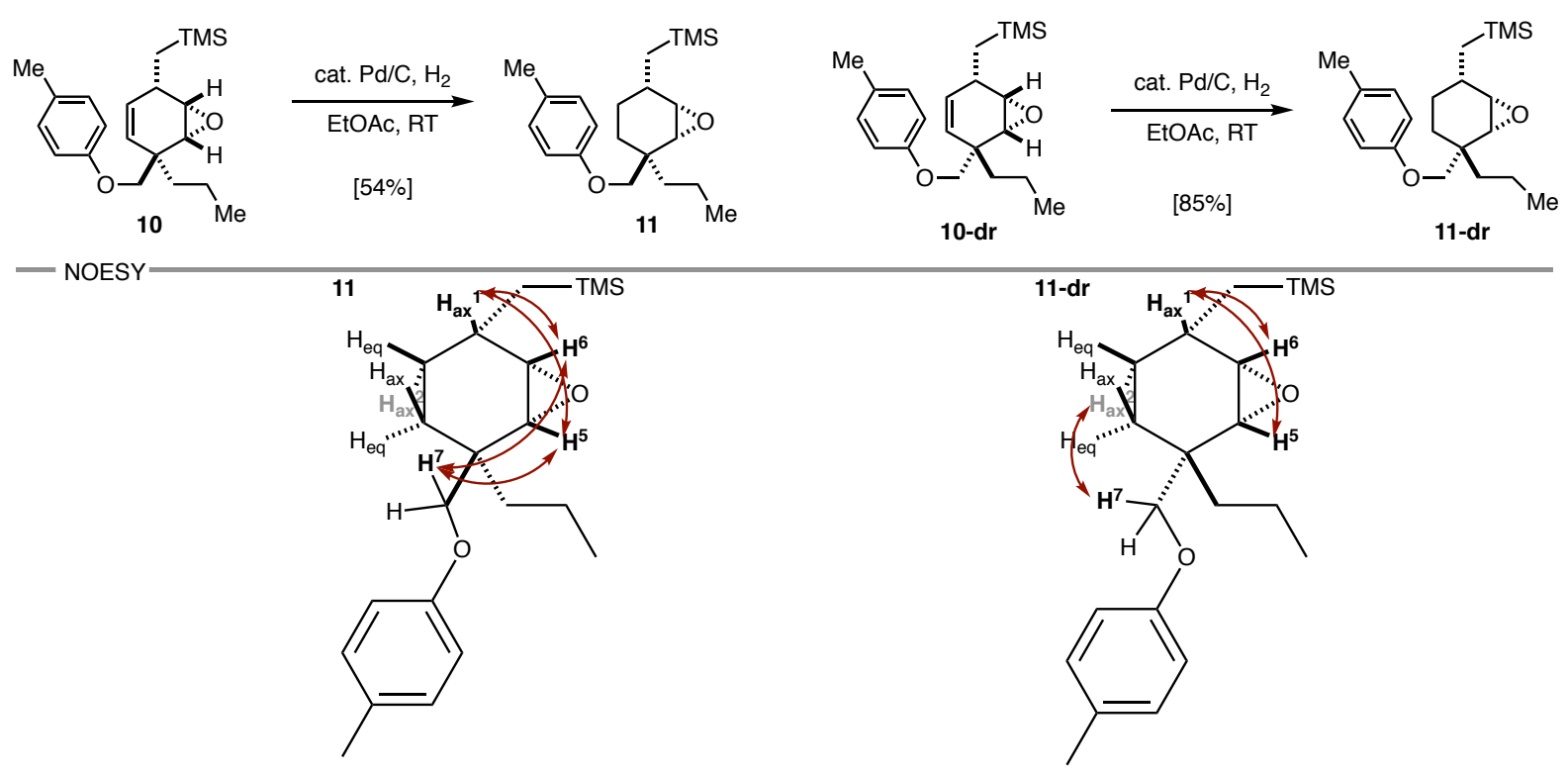


Although several NMR spectroscopy analyses (COSY, NOESY) of $\mathbf{1 0}$ and 10-dr were performed, it was difficult to determine the structure of them. Therefore, we performed hydrogenation of them to the corresponding cyclohexane oxides.

\section{Procedure for Hydrogenation}

The solution of 10 or 10-dr and $\mathrm{Pd} / \mathrm{C}(10 \% / \mathrm{wt}, 30 \mathrm{~mol} \%)$ in EtOAc $(20 \mu \mathrm{M})$ was equipped with $\mathrm{H}_{2}$ balloon (1 atm) and stirred for 10 hours at room temperature. The crude mixture was passed through a pad of Celite ${ }^{\circledR}$. The mixture was concentrated in vacuo, and then purified on PTLC (hexane/EtOAc $=$ 50:1) to afford the corresponding cyclohexane oxide $\mathbf{1 1}$ and 11-dr.

11: A colorless oil (40 $\mu \mathrm{mol} \mathrm{scale;} 7.6 \mathrm{mg}, 54 \%$ yield). ${ }^{1} \mathrm{H}$ NMR (400 MHz, $\left.\mathrm{CDCl}_{3}\right) \delta 7.06(\mathrm{~d}, J=8.4$ Hz, 2H), 6.82 (d, $J=8.4 \mathrm{~Hz}, 2 \mathrm{H}), 3.87$ (d, $J=8.8 \mathrm{~Hz}, 1 \mathrm{H}), 3.71$ (d, $J=8.8 \mathrm{~Hz}, 1 \mathrm{H}), 3.04$ (d, $J=4.0$ $\mathrm{Hz}, 1 \mathrm{H}), 2.95$ (d, $J=4.0 \mathrm{~Hz}, 1 \mathrm{H}), 2.28(\mathrm{~s}, 3 \mathrm{H}), 2.12-2.04(\mathrm{~m}, 1 \mathrm{H}), 1.72-1.62$ (m, 2H), 1.49 (td, $J=$ $12.4,4.8 \mathrm{~Hz}, 1 \mathrm{H}), 1.43-1.25(\mathrm{~m}, 3 \mathrm{H}), 1.20-1.12(\mathrm{~m}, 2 \mathrm{H}), 1.08-1.03(\mathrm{~m}, 1 \mathrm{H}), 0.94(\mathrm{t}, J=7.2 \mathrm{~Hz}, 3 \mathrm{H})$, $0.74(\mathrm{dd}, J=14.8,6.4 \mathrm{~Hz}, 1 \mathrm{H}), 0.62(\mathrm{dd}, J=14.8,8.8 \mathrm{~Hz}, 1 \mathrm{H}), 0.06(\mathrm{~s}, 9 \mathrm{H}):{ }^{13} \mathrm{C} \mathrm{NMR}(101 \mathrm{MHz}$, $\left.\mathrm{CDCl}_{3}\right) \delta 157.3,129.7,129.7,114.5,71.5,59.0,57.9,36.5,35.6,30.1,24.7,23.3,20.7,20.5,16.5$, $14.9,-0.8$.

11-dr: A colorless oil (20 $\mu \mathrm{mol}$ scale; $6.0 \mathrm{mg}, 85 \%$ yield). ${ }^{1} \mathrm{H}$ NMR $\left(400 \mathrm{MHz}, \mathrm{CDCl}_{3}\right) \delta 7.06(\mathrm{~d}, J=$ $8.4 \mathrm{~Hz}, 2 \mathrm{H}), 6.83(\mathrm{~d}, J=8.4 \mathrm{~Hz}, 2 \mathrm{H}), 3.90$ (d, $J=9.2 \mathrm{~Hz}, 1 \mathrm{H}), 3.72(\mathrm{~d}, J=9.2 \mathrm{~Hz}, 1 \mathrm{H}), 3.09(\mathrm{~d}, J=$ $3.6 \mathrm{~Hz}, 1 \mathrm{H}), 3.04(\mathrm{~d}, J=3.6 \mathrm{~Hz}, 1 \mathrm{H}), 2.28(\mathrm{~s}, 3 \mathrm{H}), 1.95-1.84(\mathrm{~m}, 1 \mathrm{H}), 1.67-1.25(\mathrm{~m}, 6 \mathrm{H}), 1.18-1.07$ (m, 2H), 0.93 (t, $J=7.2 \mathrm{~Hz}, 3 \mathrm{H}), 0.83$ (dd, $J=14.4,6.8 \mathrm{~Hz}, 1 \mathrm{H}), 0.64$ (dd, $J=14.4,7.6 \mathrm{~Hz}, 1 \mathrm{H}), 0.04$ (s, 9H): ${ }^{13} \mathrm{C} \mathrm{NMR}\left(101 \mathrm{MHz}, \mathrm{CDCl}_{3}\right) \delta 157.4,129.8,129.7,114.6,70.3,59.1,58.6,39.0,35.5,32.6$, 28.6, 25.2, 21.5, 20.5, 16.6, 15.0,-0.6. 


\section{$m$ CPBA Oxidation of $4 \mathrm{~S}$}

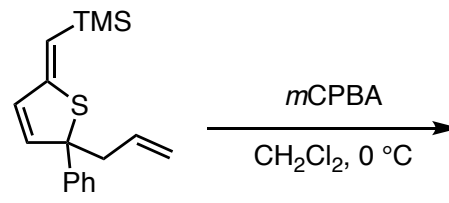

$4 S$

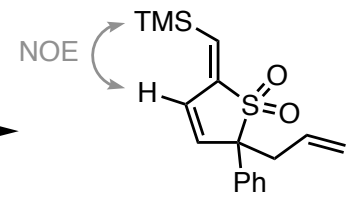

11-1

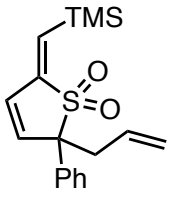

$11-2$

To a crude mixture of $4 \mathrm{~S}$ (NMR yield was $65 \%$ ) in a $20 \mathrm{~mL}$ drum-vial with a magnetic stirring bar was added $\mathrm{CH}_{2} \mathrm{Cl}_{2}(1.0 \mathrm{~mL})$. The mixture was cooled at $0{ }^{\circ} \mathrm{C}$, and then $m$-chloroperbenzoic acid ( $m$ CPBA, 77\%: $112 \mathrm{mg}, 0.50 \mathrm{mmol}, 2.5$ equiv based on the amount of 1S) was added. The mixture was stirred for $4 \mathrm{~h}$ at room temperature. The mixture was quenched with $\mathrm{NaHCO}_{3}$ aq. and $\mathrm{Na}_{2} \mathrm{~S}_{2} \mathrm{O}_{3}$ aq. at $0{ }^{\circ} \mathrm{C}$ and extracted three times with $\mathrm{CH}_{2} \mathrm{Cl}_{2}$. Combined organic layer was dried over $\mathrm{Na}_{2} \mathrm{SO}_{4}$, filtrated, and concentrated in vacuo. The mixture was purified by Isolera $^{\circledR}$ with a basic alumina column cartridge (hexane/EtOAc $=99: 1$ to $3: 2)$ to afford 11-1 $(19.2 \mathrm{mg}, 60 \mu \mathrm{mol}, 30 \%$ yield in 2 steps) as a yellow oil and $\mathbf{1 1 - 2}(12.3 \mathrm{mg}, 39 \mu \mathrm{mol}, 19 \%$ yield in 2 steps) as a yellow oil.

11-1: ${ }^{1} \mathrm{H}$ NMR (400 MHz, $\left.\mathrm{CDCl}_{3}\right) \delta 7.44-7.36(\mathrm{~m}, 5 \mathrm{H}), 6.95(\mathrm{~d}, J=8.8 \mathrm{~Hz}, 1 \mathrm{H}), 6.62(\mathrm{~d}, J=8.8 \mathrm{~Hz}$, $1 \mathrm{H}), 6.53(\mathrm{~s}, 1 \mathrm{H}), 5.60-5.49(\mathrm{~m}, 1 \mathrm{H}), 5.15-5.06(\mathrm{~m}, 2 \mathrm{H}), 3.35(\mathrm{dd}, J=14.0,6.0 \mathrm{~Hz}, 1 \mathrm{H}), 2.80(\mathrm{dd}, J=$ 14.0, $8.0 \mathrm{~Hz}, 1 \mathrm{H}), 0.25(\mathrm{~s}, 9 \mathrm{H}) ;{ }^{13} \mathrm{C} \mathrm{NMR}\left(101 \mathrm{MHz}, \mathrm{CDCl}_{3}\right) \delta 151.6,136.8,133.1,132.6,131.5$, $128.8,128.1,126.2,120.0,69.3,40.5,-0.6$ (one peak is missing due to overlapping); HRMS (DART) $m / z$ calcd for $\mathrm{C}_{17} \mathrm{H}_{26} \mathrm{O}_{2} \mathrm{NSSi}\left[\mathrm{M}+\mathrm{NH}_{4}\right]^{+}: 336.1448$ found 336.1441 .

11-2: ${ }^{1} \mathrm{H}$ NMR $\left(400 \mathrm{MHz}, \mathrm{CDCl}_{3}\right) \delta$ 7.43-7.35 (m, 5H), $6.65(\mathrm{~d}, J=8.8 \mathrm{~Hz}, 1 \mathrm{H}), 6.45(\mathrm{~d}, J=8.8 \mathrm{~Hz}$, $1 \mathrm{H}), 6.32(\mathrm{~s}, 1 \mathrm{H}), 5.59-5.49(\mathrm{~m}, 1 \mathrm{H}), 5.15-5.05(\mathrm{~m}, 2 \mathrm{H}), 3.33(\mathrm{dd}, J=14.4,6.4 \mathrm{~Hz}, 1 \mathrm{H}), 2.83(\mathrm{dd}, J=$ 14.4, 8.0 Hz, 1H), 0.27 (s, 9H); ${ }^{13} \mathrm{C}$ NMR (101 MHz, $\left.\mathrm{CDCl}_{3}\right) \delta 152.6,135.4,134.5,133.4,131.7$, $130.1,128.8,128.0,119.9,71.2,40.9,-0.4$ (one peak is missing due to overlapping); HRMS (DART) $m / z$ calcd for $\mathrm{C}_{17} \mathrm{H}_{26} \mathrm{O}_{2} \mathrm{NSSi}\left[\mathrm{M}+\mathrm{NH}_{4}\right]^{+}: 336.1448$ found 336.1442 . 


\section{Effect of Parameters}

\section{Screening of Ligands}<smiles>Cc1ccc(Br)c2ccccc12</smiles>

$1 \mathrm{~A}(0.20 \mathrm{mmol})$

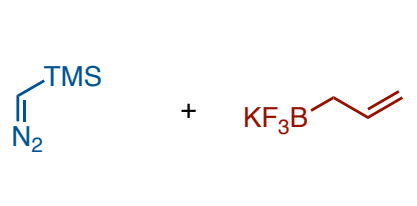

2 (1.0 equiv)

3 (1.0 equiv)

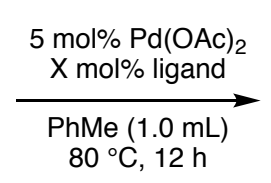

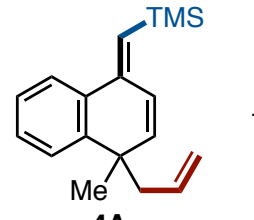

$4 \mathrm{~A}$<smiles>C=CCc1ccc(C)c2ccccc12</smiles>

$4 A^{\prime}$

entry ligand $\left(X\right.$ mol\%) yield of $4 A^{a}(\%)$ yield of $4 A^{\prime a}(\%)$ yield of $1 A^{a}(\%)$

\begin{tabular}{|c|c|c|c|c|}
\hline 1 & $\mathrm{PPh}_{3}(10)$ & 19 & 16 & 54 \\
\hline 2 & L1 (10) & 36 & 0 & 41 \\
\hline 3 & L2 (10) & 0 & 6 & 51 \\
\hline 4 & L3 (10) & 16 & 9 & 43 \\
\hline 5 & L4 (10) & 30 & 3 & 47 \\
\hline 6 & L5 (10) & 6 & 23 & 61 \\
\hline 7 & L6 (10) & 0 & 17 & 49 \\
\hline 8 & L7 (10) & 2 & 21 & 52 \\
\hline 9 & L8 (10) & 0 & 19 & 46 \\
\hline 10 & L9 (10) & 15 & 10 & 42 \\
\hline 11 & L10 (10) & 17 & 10 & 49 \\
\hline 12 & L11 (10) & 20 & 8 & 40 \\
\hline 13 & L12 (10) & 0 & 5 & 59 \\
\hline 14 & L13 (10) & 28 & 0 & 39 \\
\hline 15 & L14 (10) & 2 & 17 & 54 \\
\hline 16 & L15 (10) & 7 & 14 & 46 \\
\hline 17 & L16 (10) & 0 & 8 & 49 \\
\hline 18 & L17 (10) & 0 & 14 & 58 \\
\hline 19 & $\mathrm{PPh}_{2} \mathrm{Me}(10)$ & 26 & 2 & 53 \\
\hline 20 & $\mathrm{PPh}_{2} \mathrm{Et}(10)$ & 25 & 2 & 51 \\
\hline 21 & $\mathrm{PPh}_{2} \operatorname{Pr}(10)$ & 20 & 5 & 59 \\
\hline 22 & $\mathrm{PCy}{ }_{2} \mathrm{Ph}(10)$ & 13 & 9 & 57 \\
\hline 23 & $\mathrm{PCy}_{3} \cdot \mathrm{HBF}_{4}(10)^{\mathrm{b}}$ & 10 & 0 & 77 \\
\hline 24 & $\mathrm{P}^{n} \mathrm{Bu}_{3}(10)$ & 25 & trace & 40 \\
\hline 25 & $\mathrm{P}^{t} \mathrm{Bu}_{3} \cdot \mathrm{HBF}_{4}(10)^{\mathrm{b}}$ & 0 & 0 & 52 \\
\hline 26 & XPhos (10) & 2 & 17 & 79 \\
\hline 27 & DPEphos (5) & 2 & 21 & 53 \\
\hline 28 & $\mathrm{P}(\mathrm{OPh})_{3}(10)$ & 0 & 21 & 85 \\
\hline 29 & 1,10-phen (10) & 0 & 11 & 56 \\
\hline 30 & none & 0 & 3 & 57 \\
\hline 31 & L1 (5) & 5 & 22 & 63 \\
\hline 32 & L1 (15) & 30 & 2 & 58 \\
\hline 33 & L1 (20) & 39 & 0 & 46 \\
\hline
\end{tabular}

[a] Yields were determined by ${ }^{1} \mathrm{H}$ NMR using $\mathrm{CH}_{2} \mathrm{Br}_{2}$ as an internal standard. [b] $10 \mathrm{~mol} \% \mathrm{NaO}$ 'Bu was added.<smiles>CCCP(c1ccccc1)c1ccc(N(C)C)cc1</smiles>

L1<smiles>COc1cc(OC)c(P)c(OC)c1</smiles>

L8<smiles>CN(C)c1ccc(P(Br)CBr)cc1</smiles>

L2<smiles>Cc1cc(P)cc(P)c1</smiles>

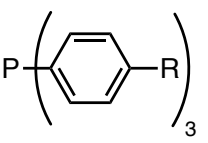

L3 $(\mathrm{R}=\mathrm{Me})$ L4 ( $\mathrm{R}=\mathrm{OMe}$ )

L9 $(\mathrm{R}=\mathrm{Me})$

L10 $\left(R={ }^{t} \mathrm{Bu}\right)$

L11 $(\mathrm{R}=\mathrm{OMe})$

$\mathrm{L} 12\left(\mathrm{R}=\mathrm{CF}_{3}\right)$

L14 ( $\mathrm{R}=$ mesityl)

L15 ( $\left.\mathrm{R}={ }^{\mathrm{i}} \mathrm{Pr}\right)$

L16 $(R=$ OPh $)$<smiles>[R]c1ccccc1P</smiles>

L5 $(R=M e)$ L6 $(\mathrm{R}=\mathrm{OMe})$<smiles>COc1cc(Br)c(P)c(Br)c1</smiles>

L13<smiles>COc1ccccc1P</smiles>

L7<smiles>Cc1cccc2ccccc12</smiles>

L17 


\section{Screening of Solvents}

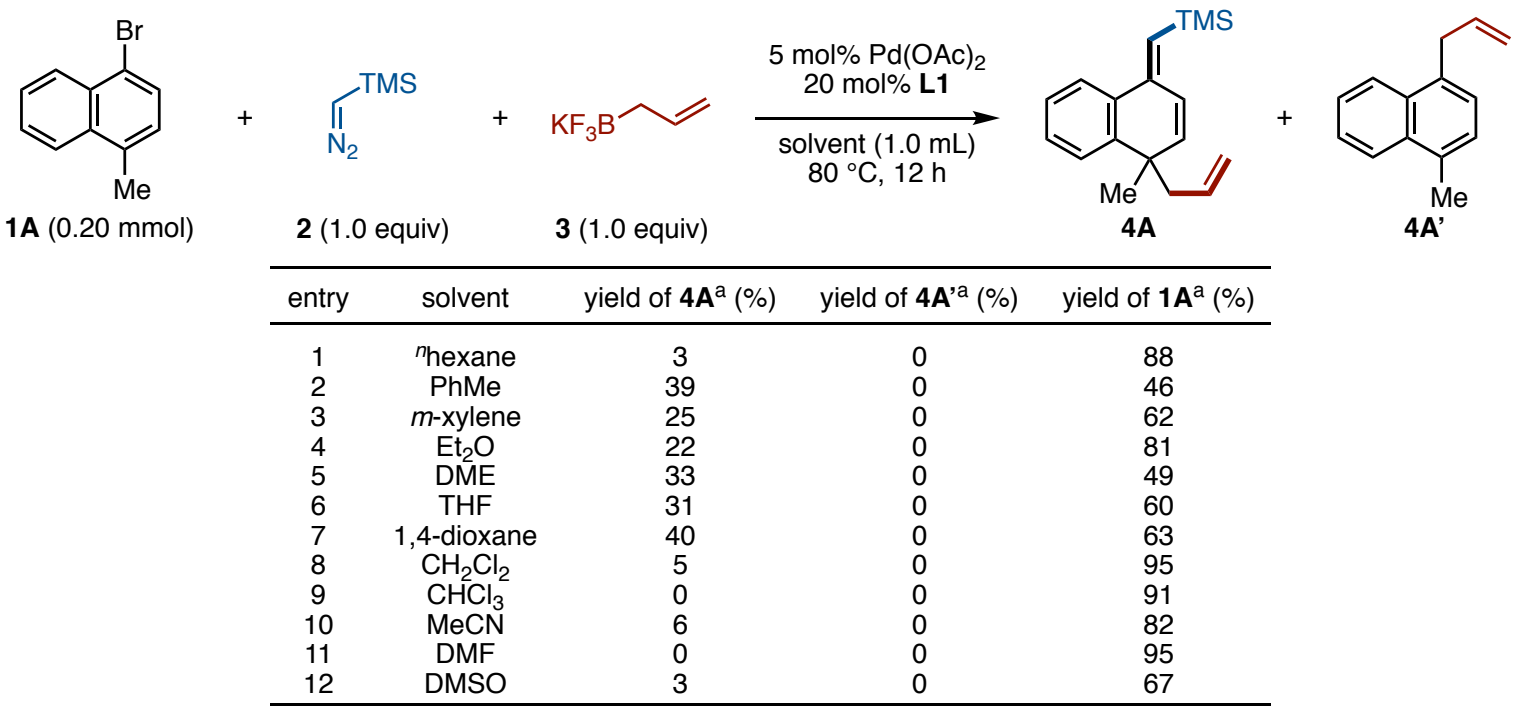

[a] Yields were determined by ${ }^{1} \mathrm{H}$ NMR using $\mathrm{CH}_{2} \mathrm{Br}_{2}$ as an internal standard.

\section{Screening of Pd Sources}<smiles>Cc1ccc(Br)c2ccccc12</smiles>

$1 \mathrm{~A}(0.20 \mathrm{mmol})$<smiles>CC(C)C=[W]</smiles>

2 (1.0 equiv)
$+\mathrm{KF}_{3} \mathrm{~B} \widehat{\gamma}$

3 (1.0 equiv)

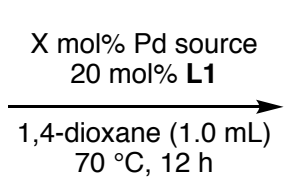
$70^{\circ} \mathrm{C}, 12 \mathrm{~h}$<smiles>C=CCC1(C)C=CC(=CC(C)(C)C)c2ccccc21</smiles>

4A<smiles>C=CCc1ccc(C)c2ccccc12</smiles>

4A'

\begin{tabular}{ccccc}
\hline entry & $\mathrm{Pd}$ source $(\mathbf{X}$ mol\%) & yield of $\mathbf{4 A ^ { a }}(\%)$ & yield of $\mathbf{4 A ^ { \prime a }}(\%)$ & yield of $\mathbf{1 A}^{\mathrm{a}}(\%)$ \\
\hline 1 & $\mathrm{Pd}(\mathrm{OAc})_{2}(5)$ & 46 & 0 & 58 \\
2 & $\mathrm{Pd}(\mathrm{COOCF})_{2}(5)$ & 39 & 0 & 53 \\
3 & $\mathrm{Pd}(\mathrm{MeCN})_{4}\left(\mathrm{BF}_{4}\right)_{2}(5)$ & 35 & 0 & 64 \\
4 & $\mathrm{Pd}(\mathrm{cod}) \mathrm{Cl}_{2}(5)$ & 21 & 0 & 73 \\
5 & $\mathrm{Pd}(\mathrm{acac})_{2}(5)$ & 32 & 0 & 48 \\
6 & $\mathrm{Pd}_{2}(\mathrm{allyl})_{2} \mathrm{Cl}_{2}(2.5)$ & 41 & 0 & 51 \\
7 & $\mathrm{Pd}(\mathrm{PhCN})_{2} \mathrm{Cl}_{2}(5)$ & 36 & 0 & 56 \\
8 & $\mathrm{Pd}_{2}(\mathrm{dba})_{3} \cdot \mathrm{CHCl}_{3}(2.5)$ & 56 & 0 & 49 \\
9 & $\mathrm{Pd}\left(\mathrm{PPh}_{3}\right)_{4}(5)$ & 35 & 0 & 54 \\
\hline
\end{tabular}

[a] Yields were determined by ${ }^{1} \mathrm{H}$ NMR using $\mathrm{CH}_{2} \mathrm{Br}_{2}$ as an internal standard. 


\section{Effect of Additives}<smiles>Cc1ccc(Br)c2ccccc12</smiles>

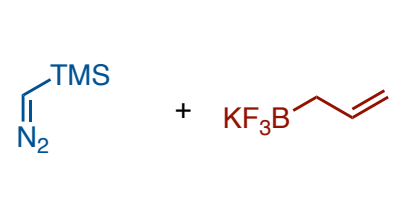

$2.5 \mathrm{~mol} \% \mathrm{Pd}_{2}(\mathrm{dba})_{3} \cdot \mathrm{CHCl}_{3}$ 20 mol\% L1 additive ( 2.0 equiv) 1,4-dioxane $(1.0 \mathrm{~mL})$ $70^{\circ} \mathrm{C}, 12 \mathrm{~h}$

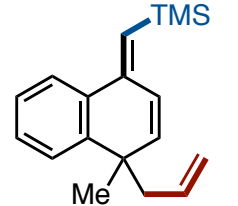

4A<smiles>C=CCc1ccc(C)c2ccccc12</smiles>

4A'

\begin{tabular}{|c|c|c|c|c|}
\hline entry & additive & yield of $\mathbf{4} A^{a}(\%)$ & yield of $4 A^{\prime a}(\%)$ & yield of $1 A^{a}(\%)$ \\
\hline 1 & none & 56 & 0 & 49 \\
\hline 2 & $\mathrm{LiF}$ & 44 & 0 & 48 \\
\hline 3 & $\mathrm{LiCl}$ & 11 & 0 & 88 \\
\hline 4 & $\mathrm{LiBr}$ & 0 & 0 & 97 \\
\hline 5 & Lil & 0 & 0 & 100 \\
\hline 6 & $\mathrm{NaF}$ & 65 & 0 & 42 \\
\hline 7 & $\mathrm{NaCl}$ & 57 & 0 & 49 \\
\hline 8 & $\mathrm{NaBr}$ & 56 & 0 & 50 \\
\hline 9 & $\mathrm{Nal}$ & 0 & 0 & 57 \\
\hline 10 & $\mathrm{KF}$ & 84 & 0 & 11 \\
\hline 11 & $\mathrm{KCl}$ & 54 & 0 & 50 \\
\hline 12 & $\mathrm{KBr}$ & 45 & 0 & 40 \\
\hline 13 & $\mathrm{KI}$ & 46 & 0 & 51 \\
\hline 14 & $\mathrm{CsF}$ & 7 & 0 & 63 \\
\hline 15 & $\mathrm{CsCl}$ & 61 & 0 & 40 \\
\hline 16 & $\mathrm{CsBr}$ & 56 & 0 & 47 \\
\hline 17 & Csl & 26 & 0 & 54 \\
\hline 18 & $\mathrm{Na}_{2} \mathrm{SO}_{4}$ & 14 & 0 & 58 \\
\hline 19 & $\mathrm{MgSO}_{4}$ & 52 & 0 & 54 \\
\hline
\end{tabular}

[a] Yields were determined by ${ }^{1} \mathrm{H}$ NMR using $\mathrm{CH}_{2} \mathrm{Br}_{2}$ as an internal standard. 


\section{References}

[1] Waller, P. J.; Lyle, S. J.; Osborn Popp, T. M.; Diercks, C. S.; Reimer, J. A.; Yaghi, O. M. Chemical Conversion of Linkages in Covalent Organic Frameworks. J. Am. Chem. Soc. 2016, 138, $15519-15522$.

[2] Yang, C.; Mehmood, F.; Lam, T. L.; Chan, S. L.-F.; Wu, Y.; Yeung, C.-S.; Guan, X.; Li, K.; Chung, C. Y.-S.; Zhou, C.-Y.; Zou, T.; Che, C.-M. Stable luminescent iridium(III) complexes with bis( $N$-heterocyclic carbene) ligands: photo-stability, excited state properties, visible-light-driven radical cyclization and $\mathrm{CO}_{2}$ reduction, and cellular imaging. Chem. Sci. 2016, 7, 3123-3136.

[3] Amb, C. M.; Rasmussen, S. C. Sterics versus Electronics: Regioselective Cross-Coupling of Polybrominated Thiophenes. Eur. J. Org. Chem. 2008, 5, 801-804.

[4] Ni, S.; Remaily, El, M. A. E. A. A. A.; Franzén, J. Carbocation Catalyzed Bromination of Alkyl Arenes, a Chemoselective sp ${ }^{3}$ vs. sp $^{2}$ C-H functionalization. Adv. Synth. Catal. 2018, 360, 4197-4204. [5] Wu, G.; Deng, Y.; Wu, C.; Zhang, Y.; Wang, J. Synthesis of $\alpha$-Aryl Esters and Nitriles: Deaminative Coupling of $\alpha$-Aminoesters and $\alpha$-Aminoacetonitriles with Arylboronic Acids. Angew. Chem., Int. Ed. 2014, 53, 10510-10514.

[6] Heller, S. T.; Sarpong, R. Chemoselective Esterification and Amidation of Carboxylic Acids with Imidazole Carbamates and Ureas. Org. Lett. 2010, 12, 4572-4575.

[7] Mao, H.; Wang, S.; Yu, P.; Lv, H.; Xu, R.; Pan, Y. Brønsted Acid-Catalyzed Decarboxylative Redox Amination: Formation of $\mathrm{N}$-Alkylindoles from Azomethine Ylides by Isomerization. J. Org. Chem. 2011, 76, 1167-1169.

[8] Boehm, J. C.; Busch-Peterson, J.; Fu, W.; Jin, Q.; Kerns, J. K.; Li, H.; Lin, G.; Lin, X.; Neipp, C. E. Indole carboxamides as ikk2 inhibitors WO2008118724A1, 03-20-2008, 2008. 


\section{9. ${ }^{1} \mathrm{H}$ and ${ }^{13} \mathrm{C}$ NMR Spectra}

${ }^{1} \mathrm{H}$ NMR of 1 B $\left(400 \mathrm{MHz}, \mathrm{CDCl}_{3}\right)$

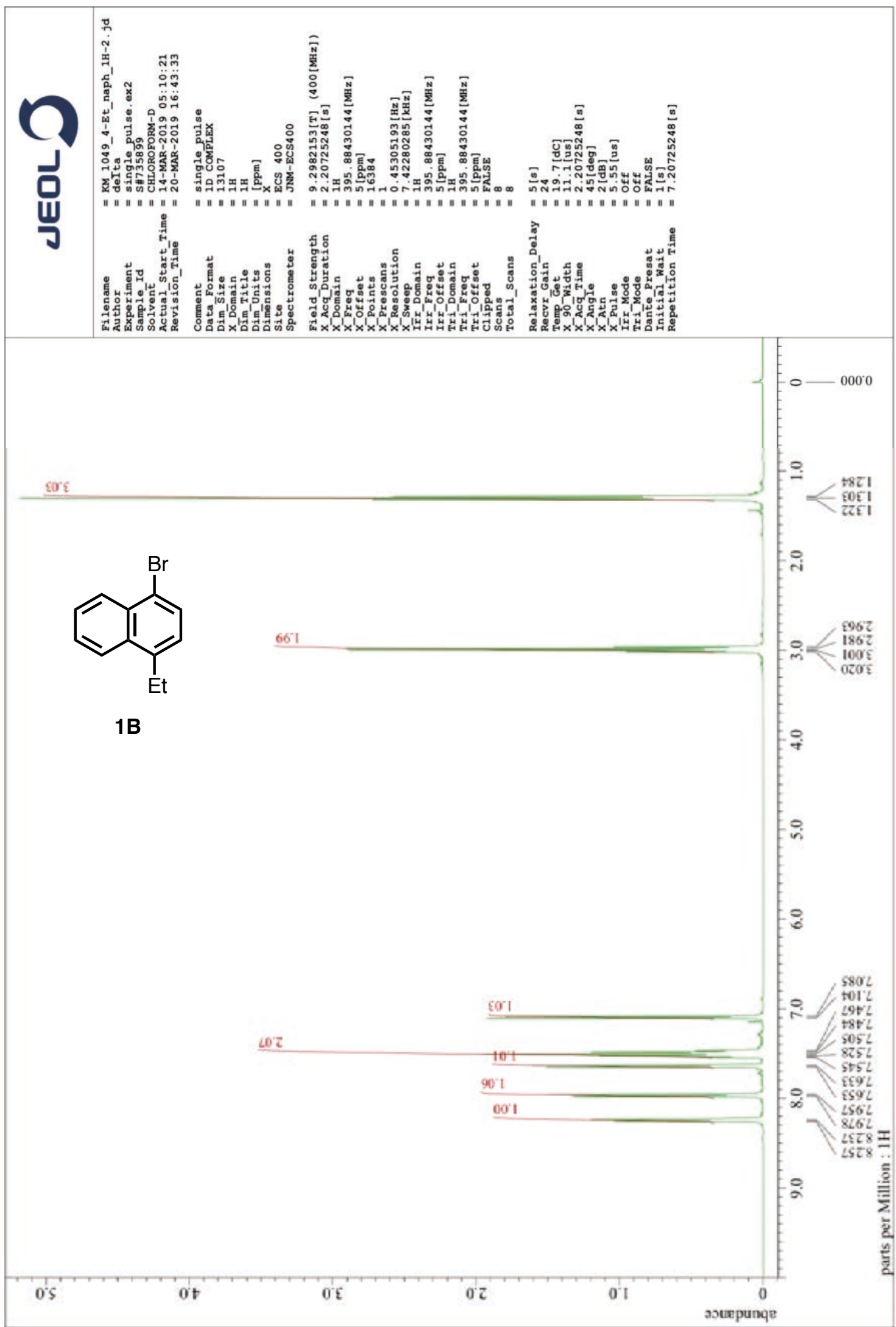


${ }^{13} \mathrm{C}$ NMR of 1B $\left(101 \mathrm{MHz}, \mathrm{CDCl}_{3}\right)$

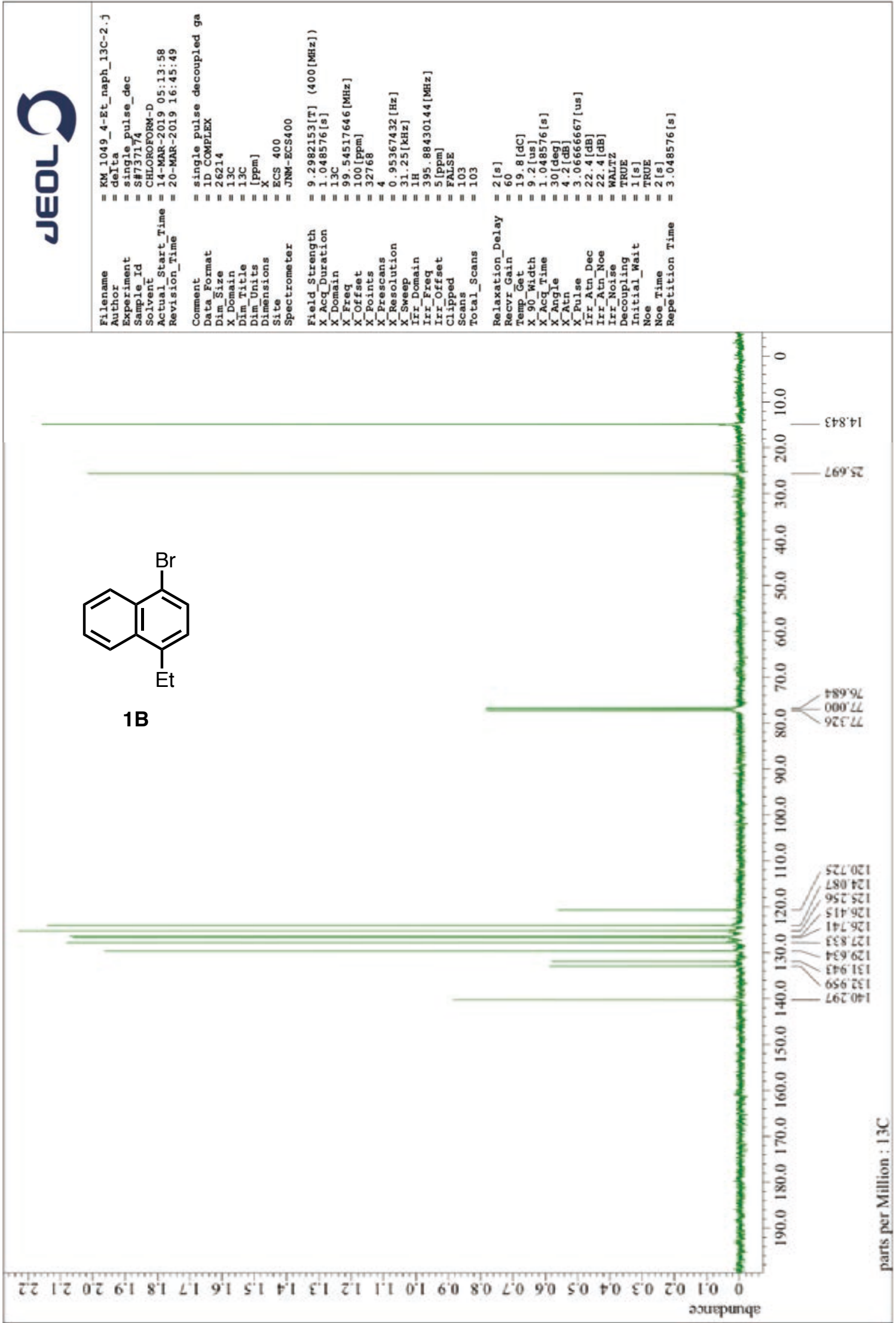


${ }^{1} \mathrm{H} \mathrm{NMR}$ of $1 \mathrm{D}\left(400 \mathrm{MHz}, \mathrm{CDCl}_{3}\right)$

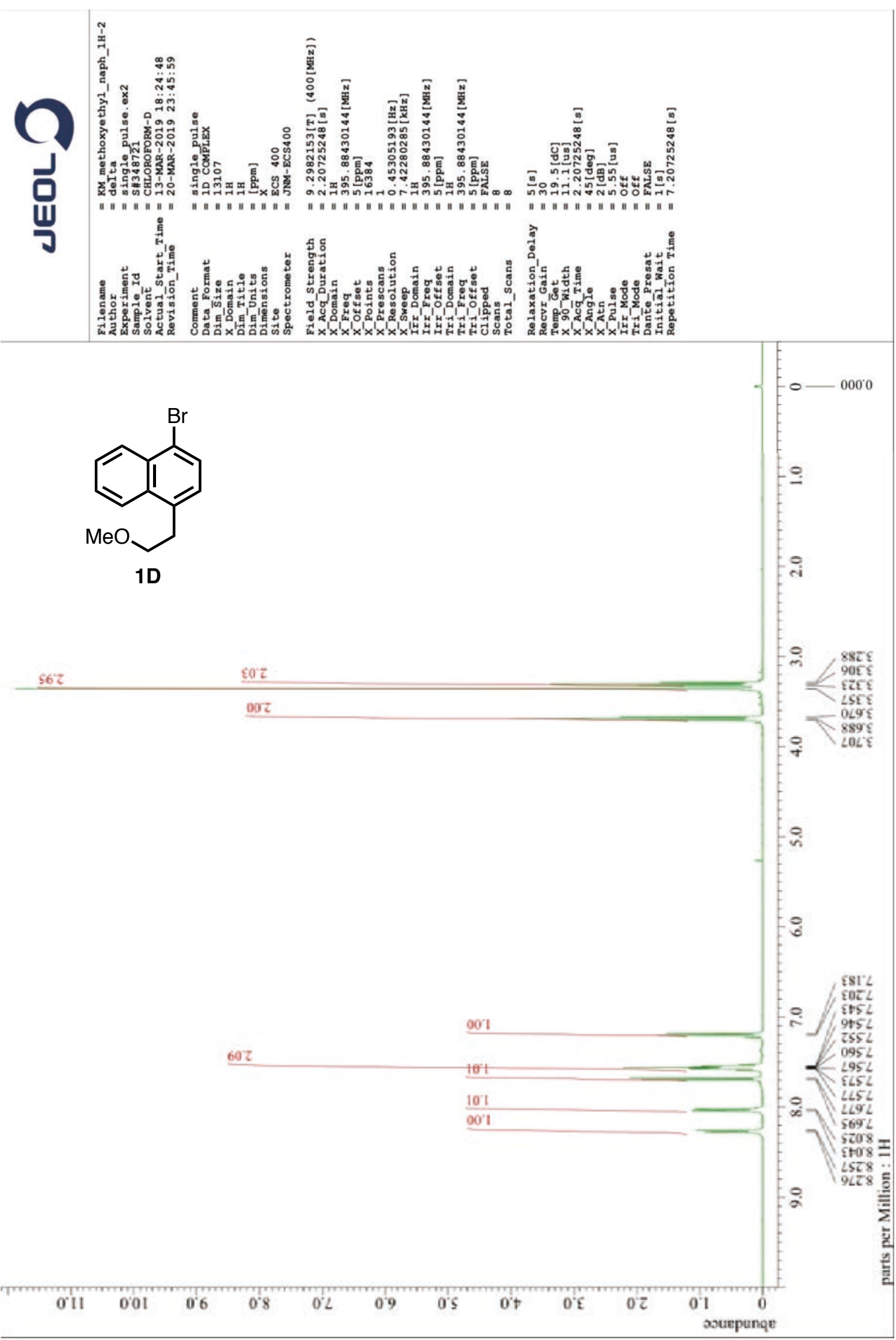


${ }^{13} \mathrm{C}$ NMR of 1D $\left(101 \mathrm{MHz}, \mathrm{CDCl}_{3}\right)$

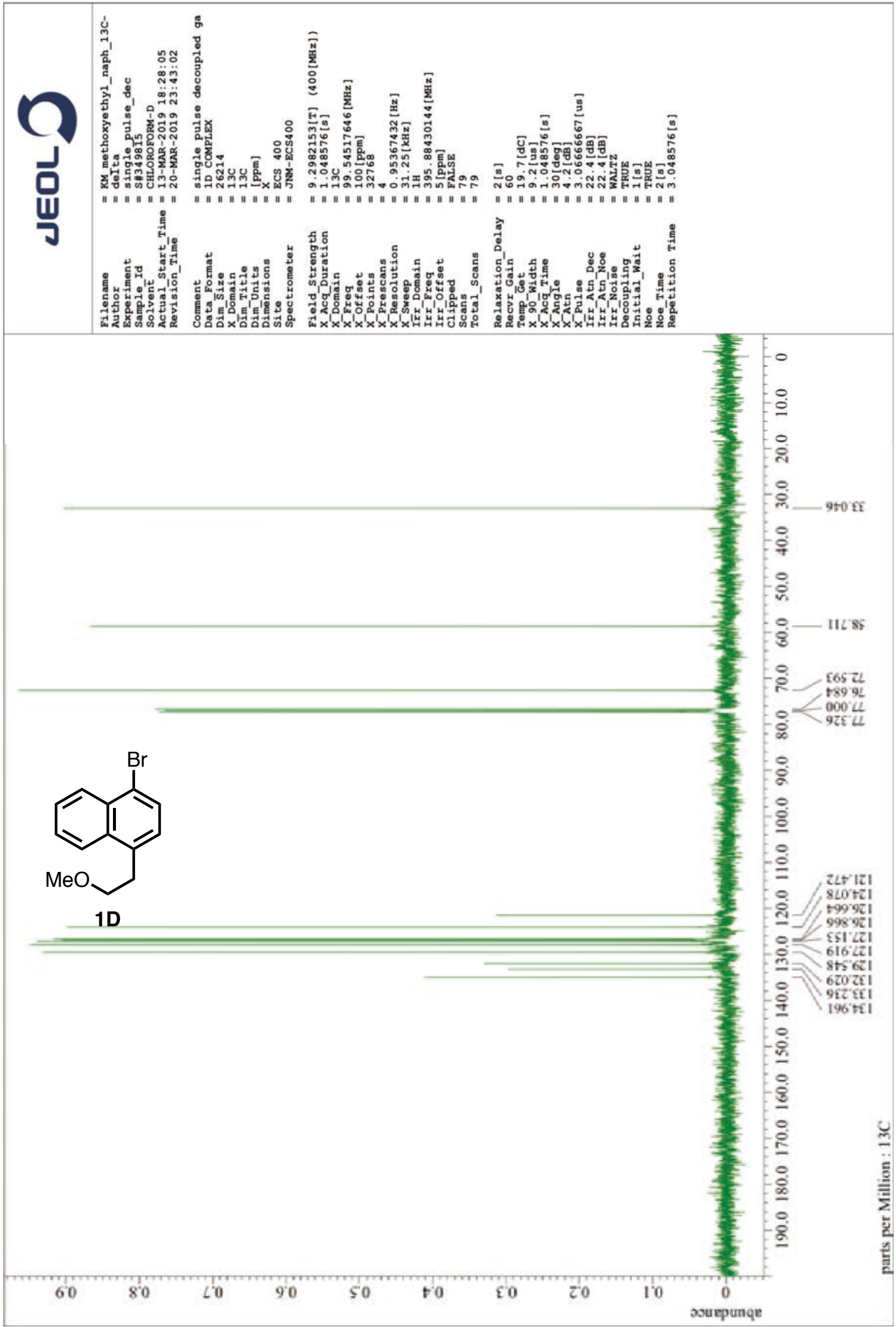


${ }^{1} \mathrm{H}$ NMR of $\mathbf{1 E}\left(400 \mathrm{MHz}, \mathrm{CDCl}_{3}\right)$

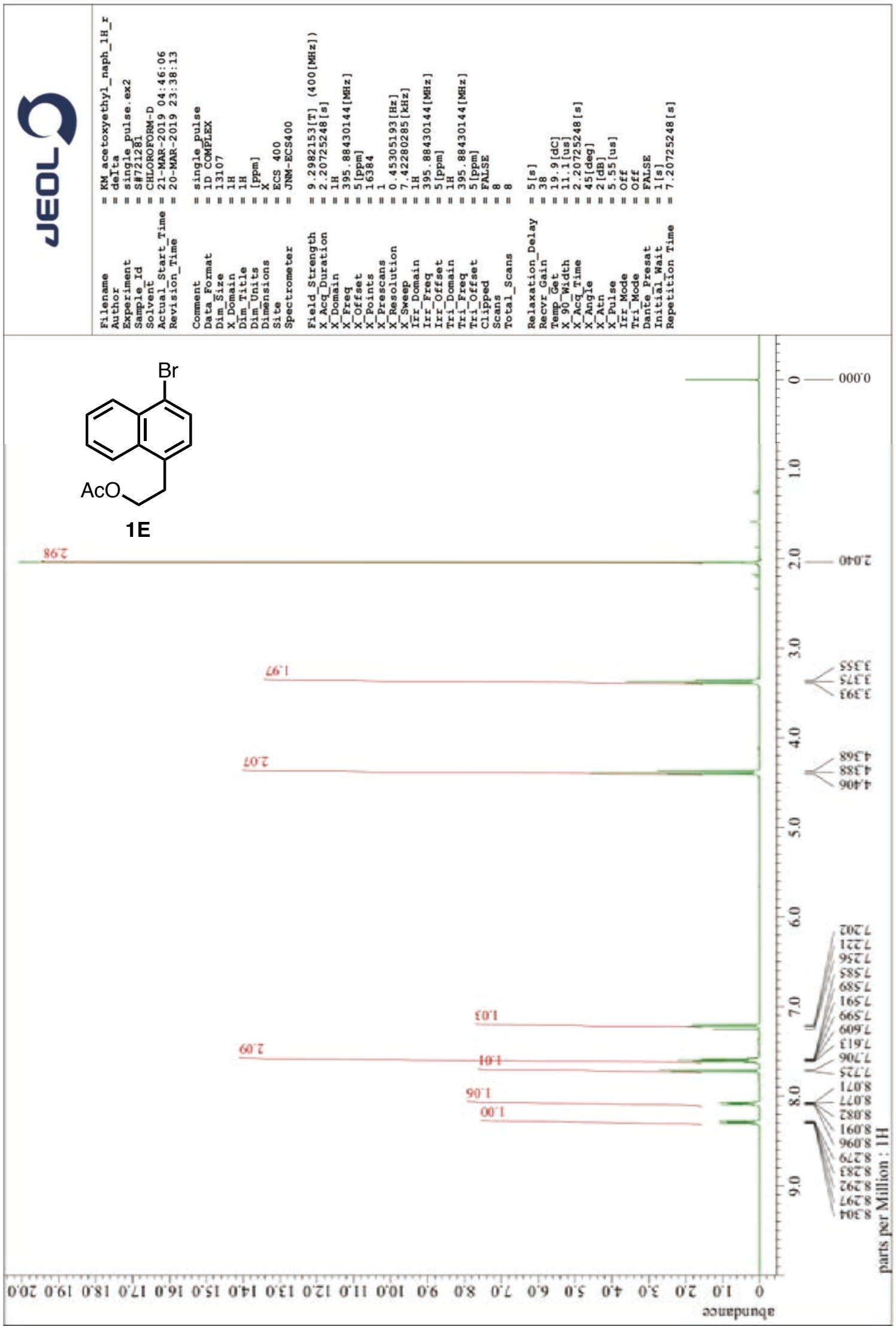


${ }^{13} \mathrm{C}$ NMR of $1 \mathbf{E}\left(101 \mathrm{MHz}, \mathrm{CDCl}_{3}\right)$

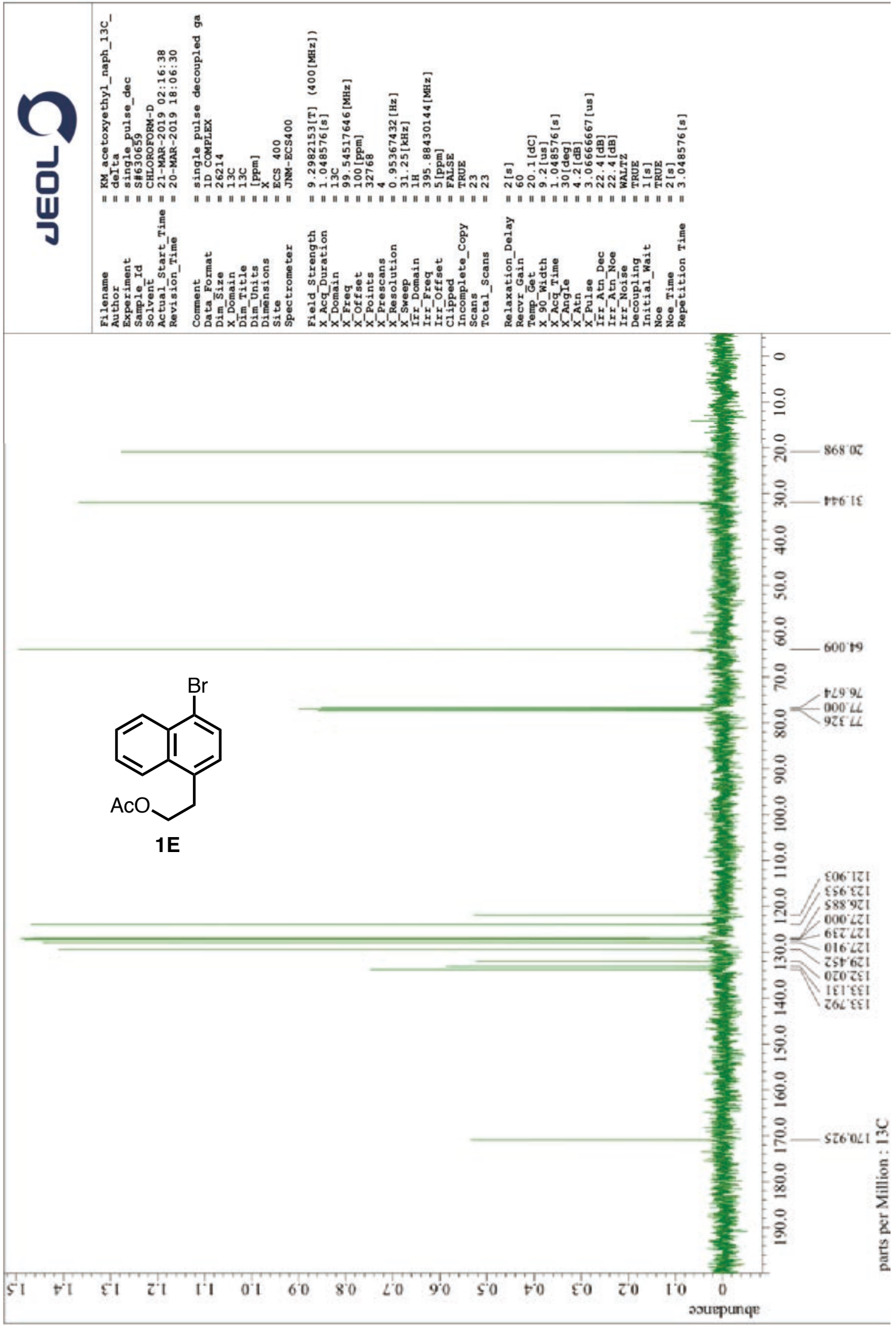


${ }^{1} \mathrm{H}$ NMR of $\mathbf{1 F}\left(400 \mathrm{MHz}, \mathrm{CDCl}_{3}\right)$

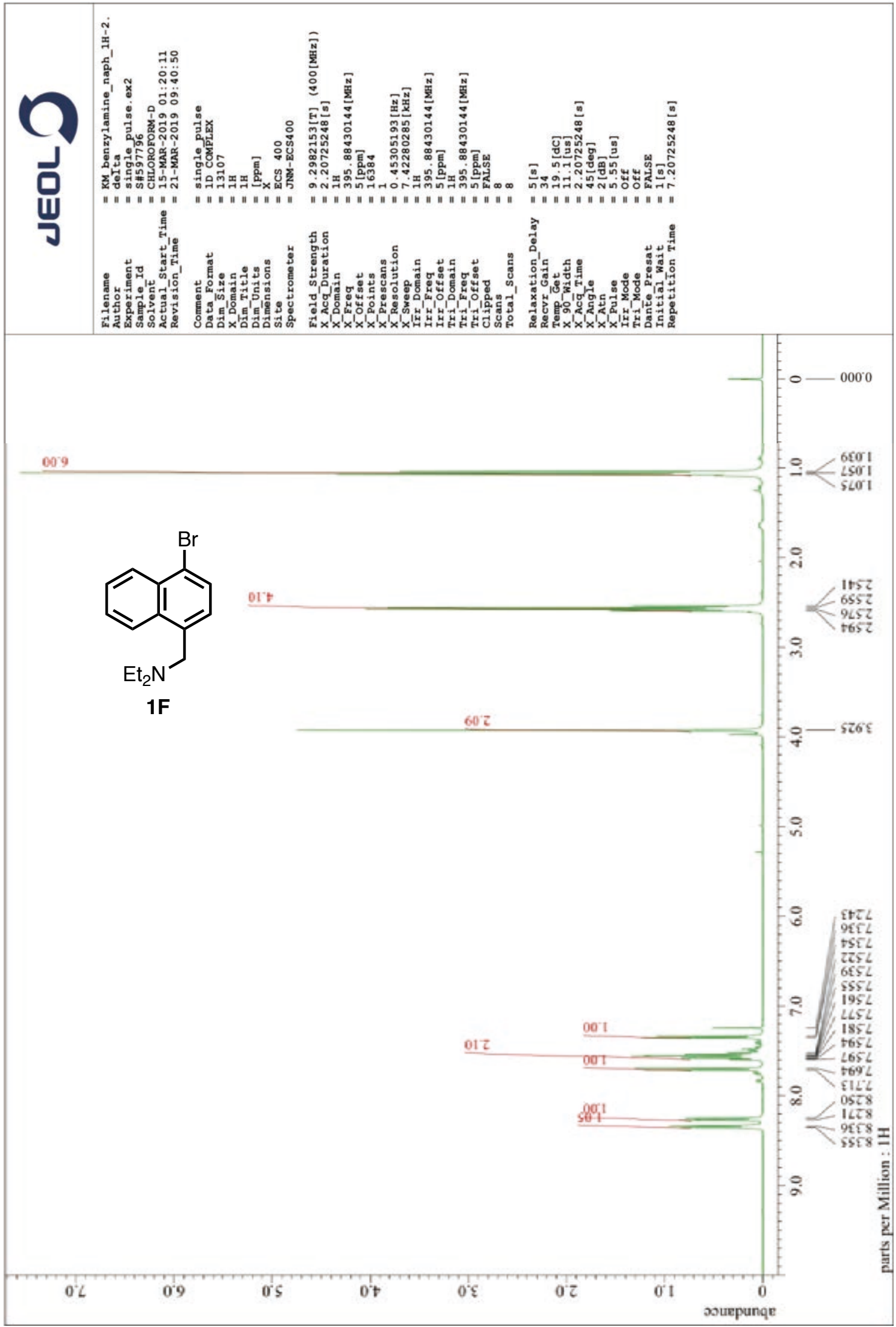


${ }^{13} \mathrm{C}$ NMR of $\mathbf{1 F}\left(101 \mathrm{MHz}, \mathrm{CDCl}_{3}\right)$

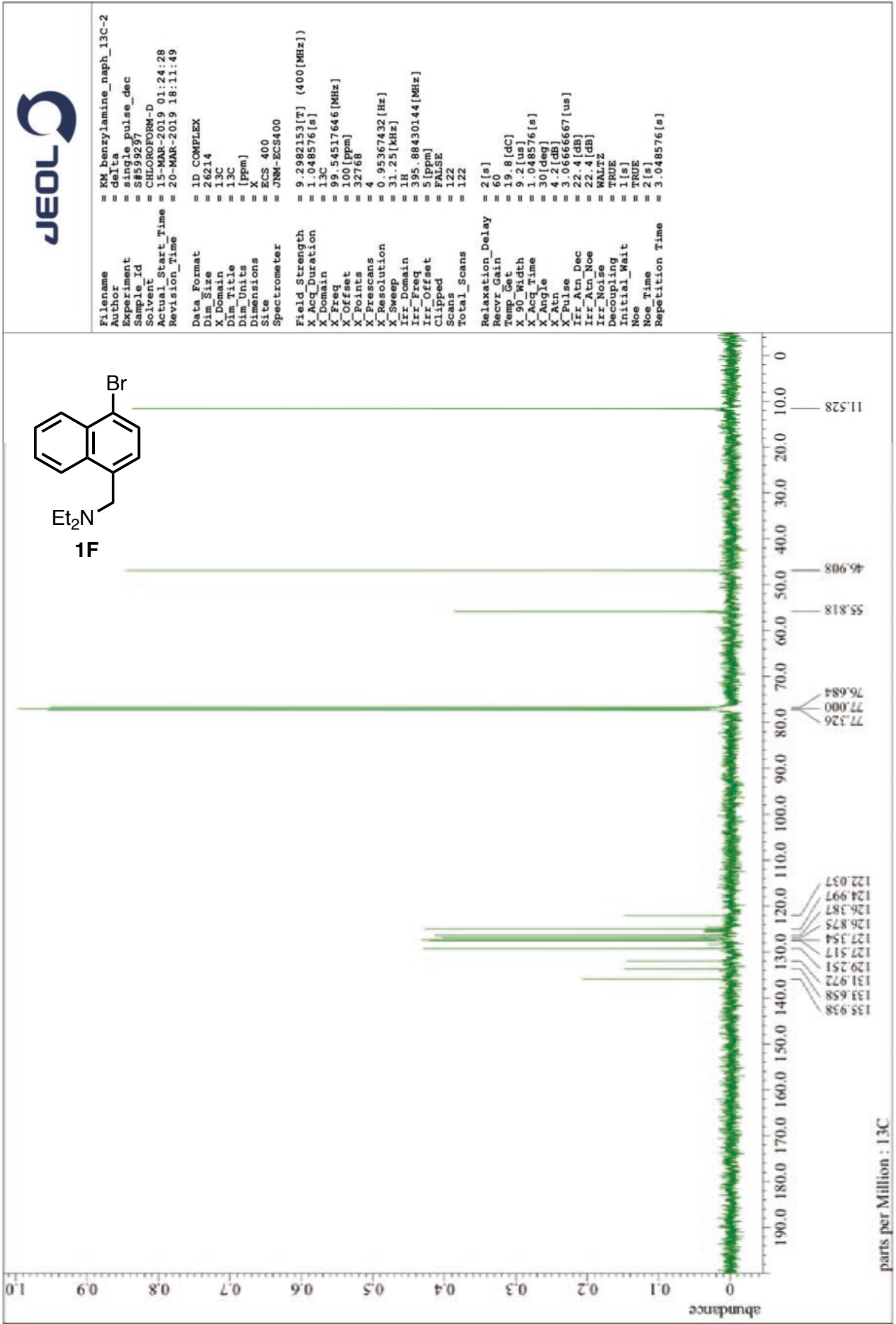


${ }^{1} \mathrm{H}$ NMR of $\mathbf{1 M}\left(400 \mathrm{MHz}, \mathrm{CDCl}_{3}\right)$

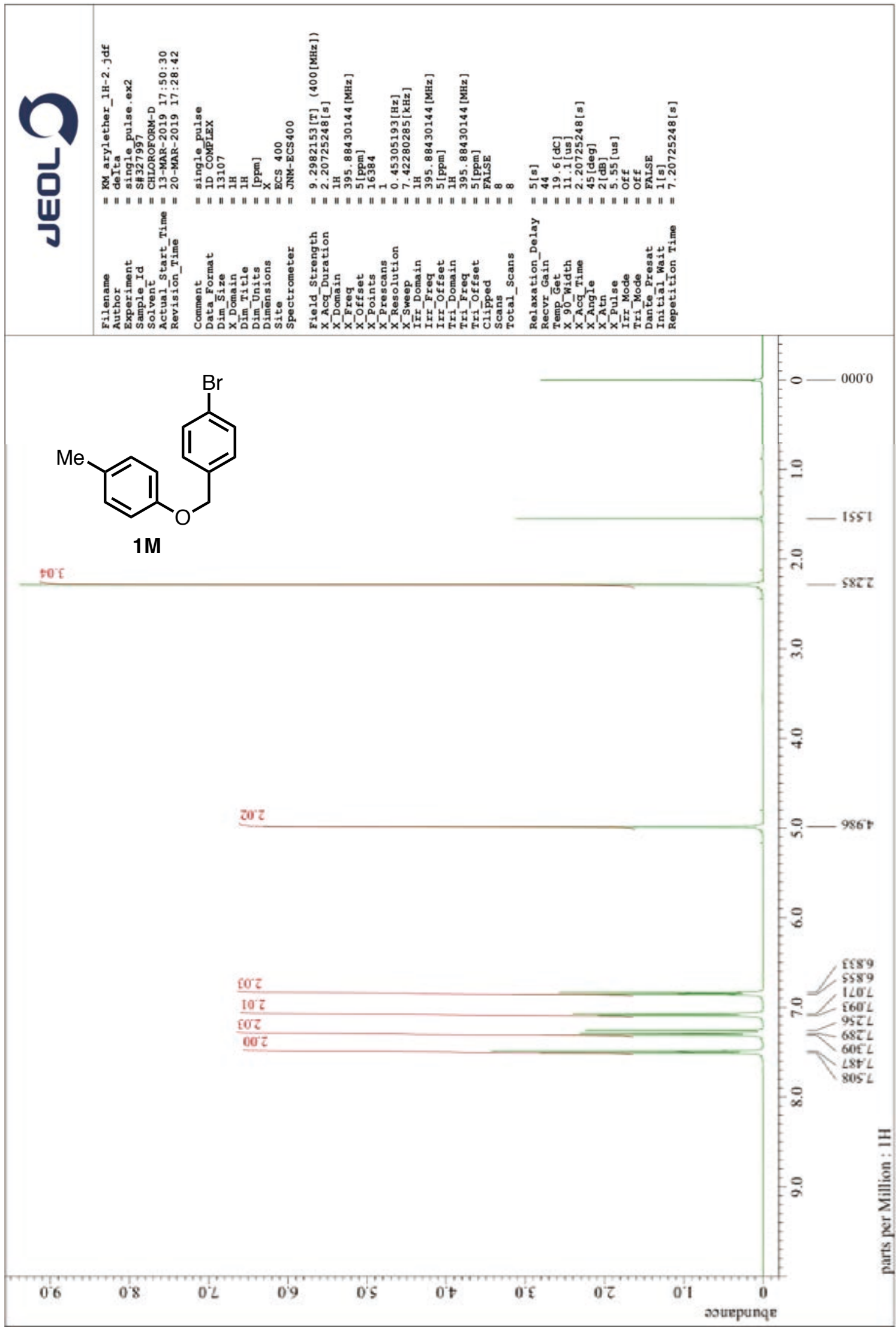


${ }^{13} \mathrm{C}$ NMR of $1 \mathbf{M}\left(101 \mathrm{MHz}, \mathrm{CDCl}_{3}\right)$

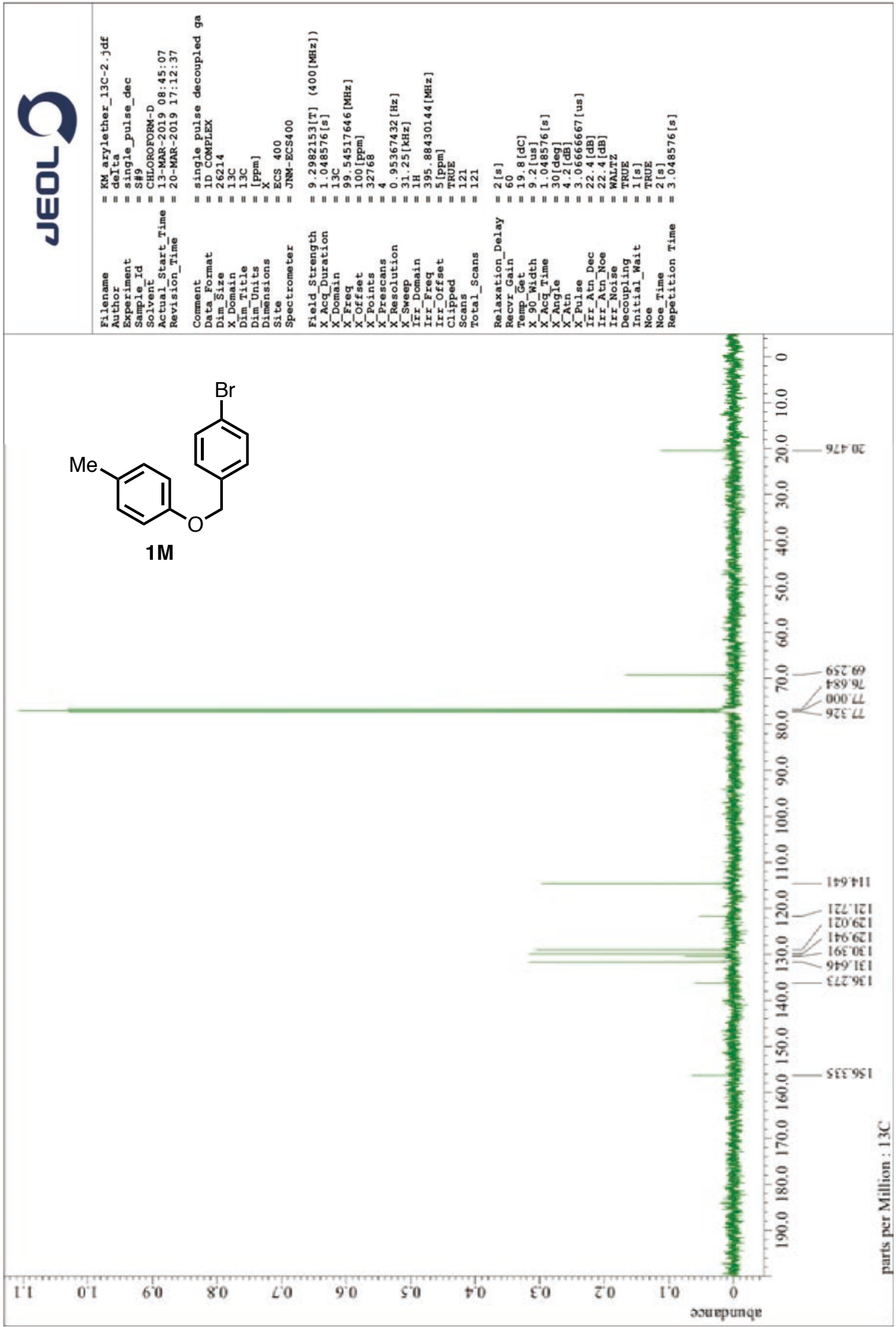


${ }^{1} \mathrm{H}$ NMR of $\mathbf{1 W}\left(400 \mathrm{MHz}, \mathrm{CDCl}_{3}\right)$

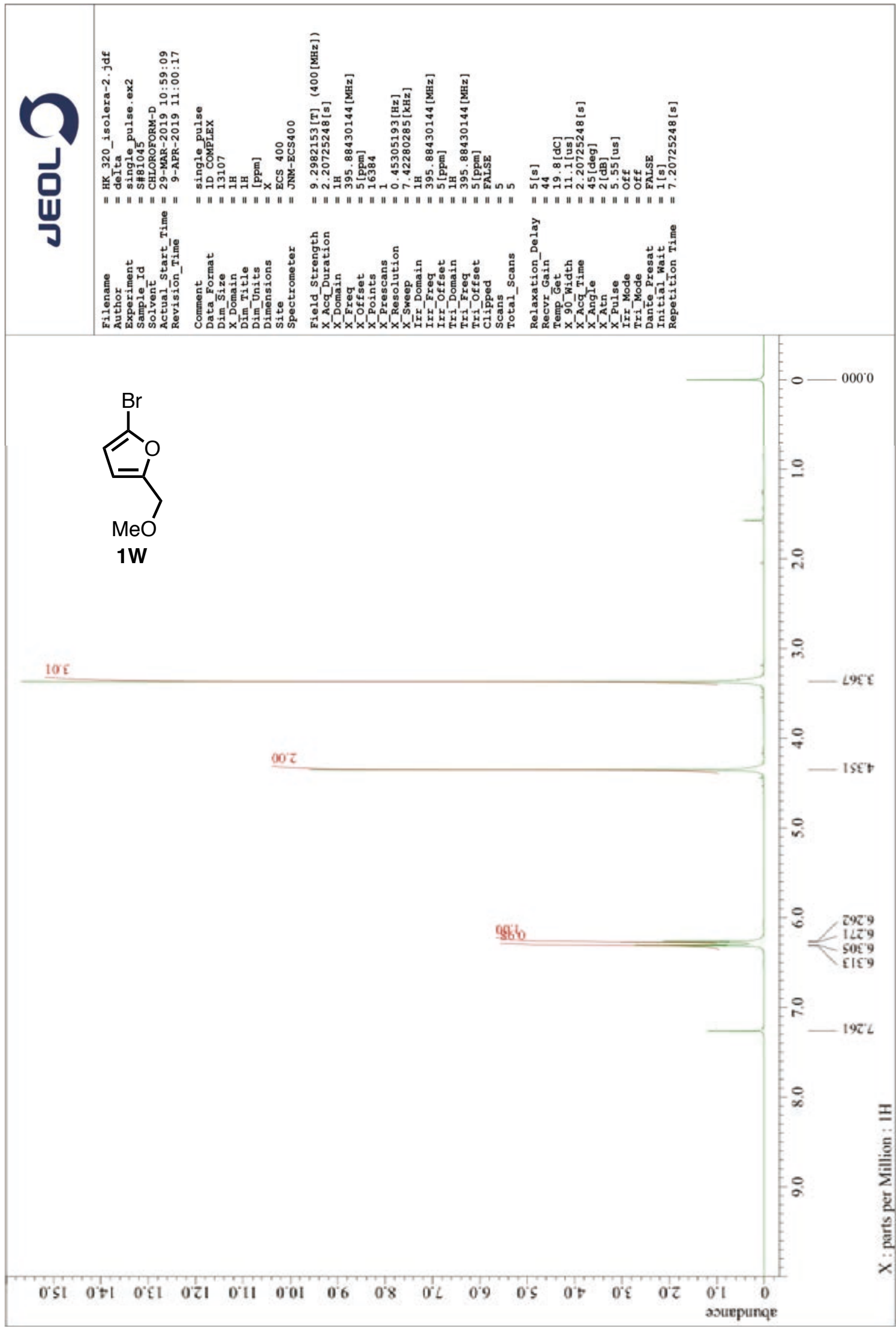


${ }^{13} \mathrm{C}$ NMR of $\mathbf{1 W}\left(101 \mathrm{MHz}, \mathrm{CDCl}_{3}\right)$

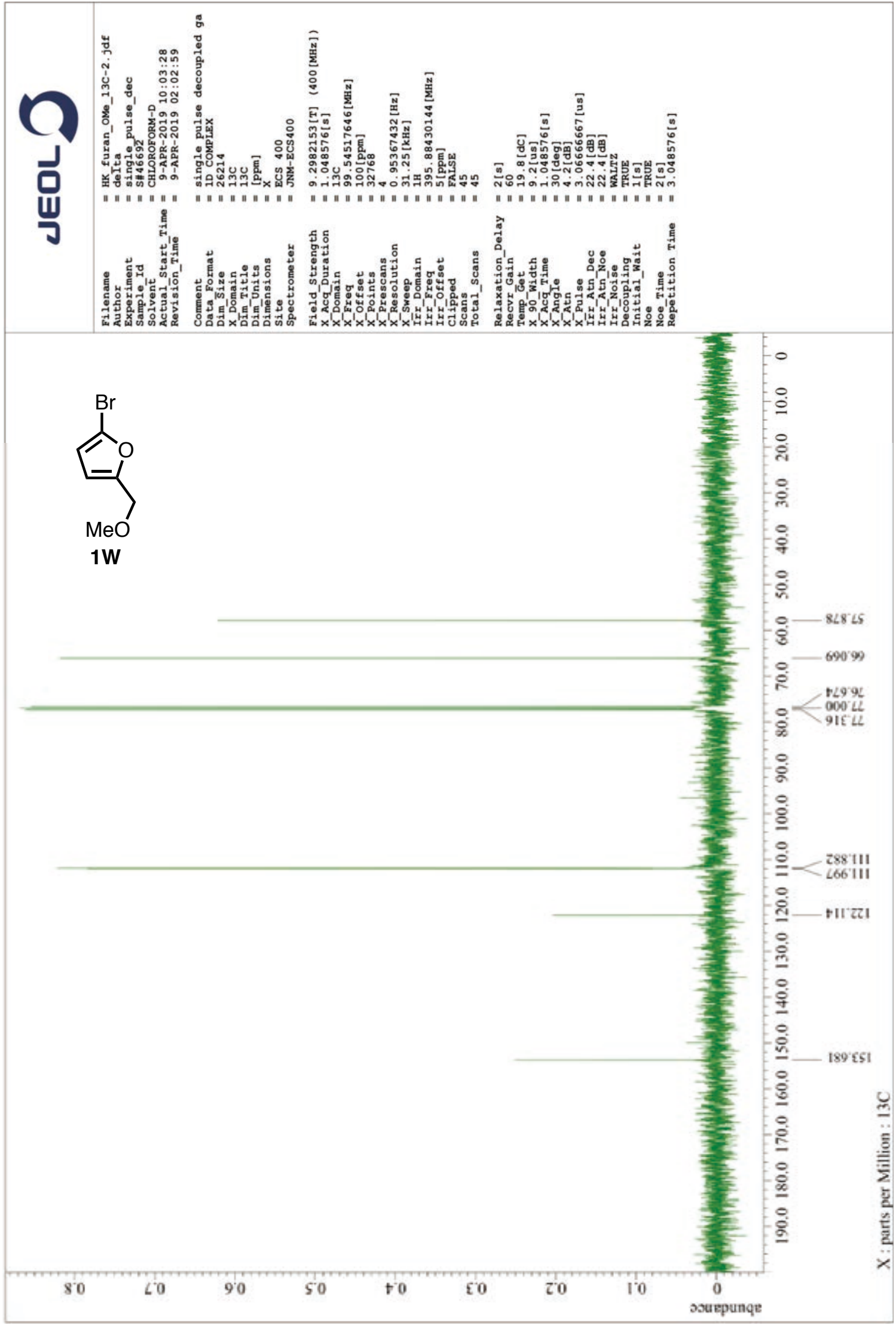


${ }^{1} \mathrm{H}$ NMR of $\mathbf{1 X}\left(400 \mathrm{MHz}, \mathrm{CDCl}_{3}\right)$

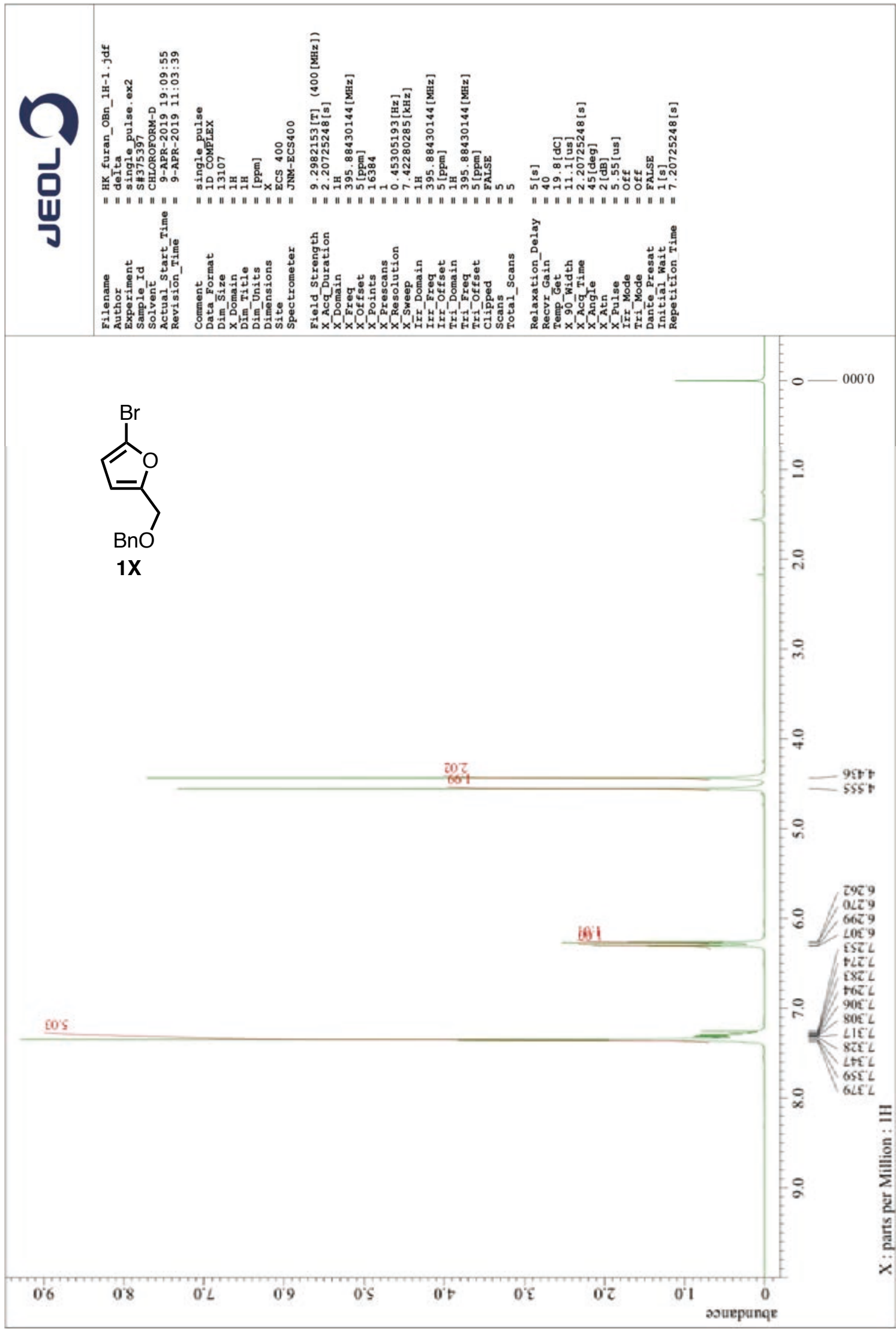


${ }^{13} \mathrm{C}$ NMR of $\mathbf{1 X}\left(101 \mathrm{MHz}, \mathrm{CDCl}_{3}\right)$

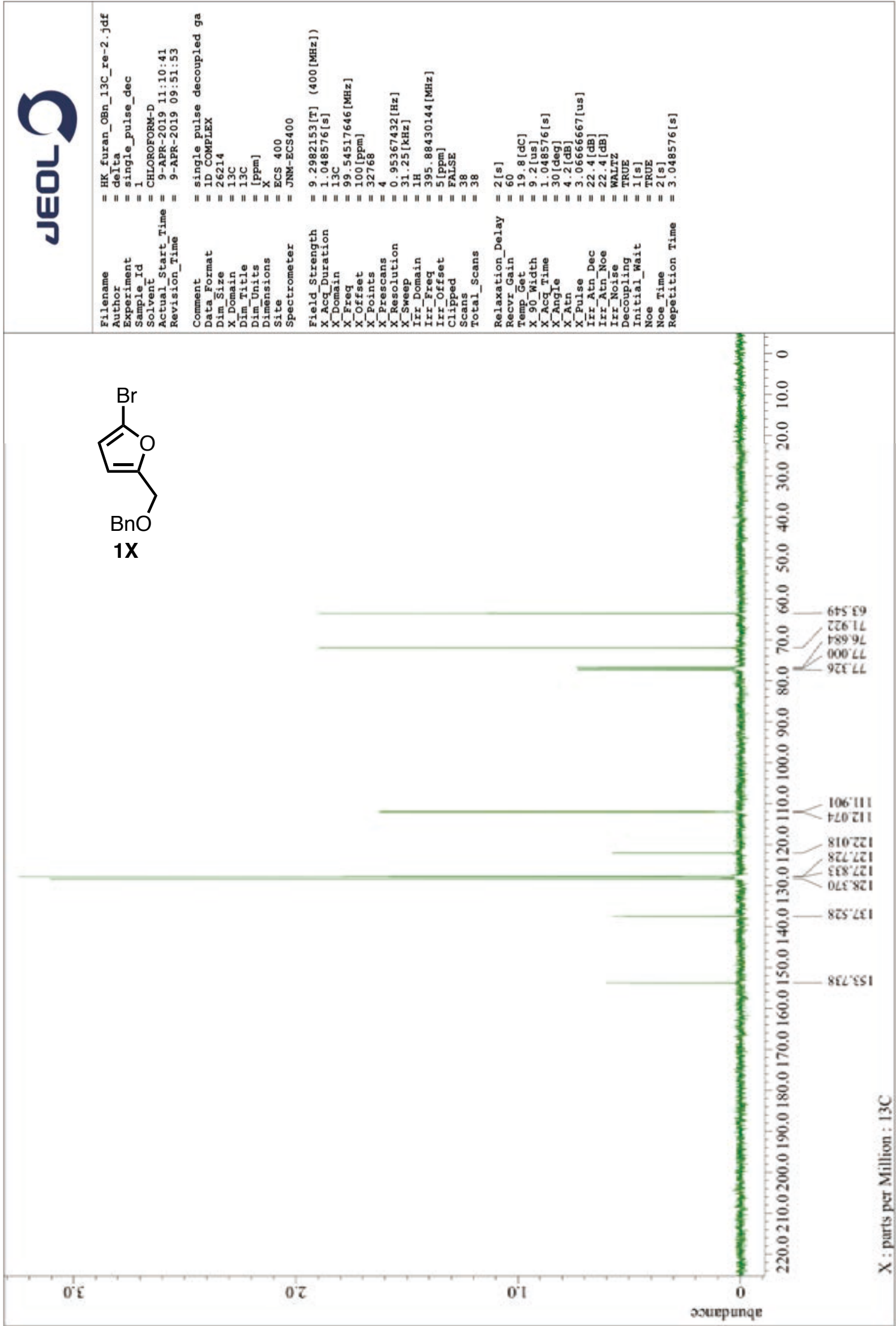


${ }^{1} \mathrm{H}$ NMR of $4 \mathbf{A}\left(400 \mathrm{MHz}, \mathrm{CDCl}_{3}\right)$

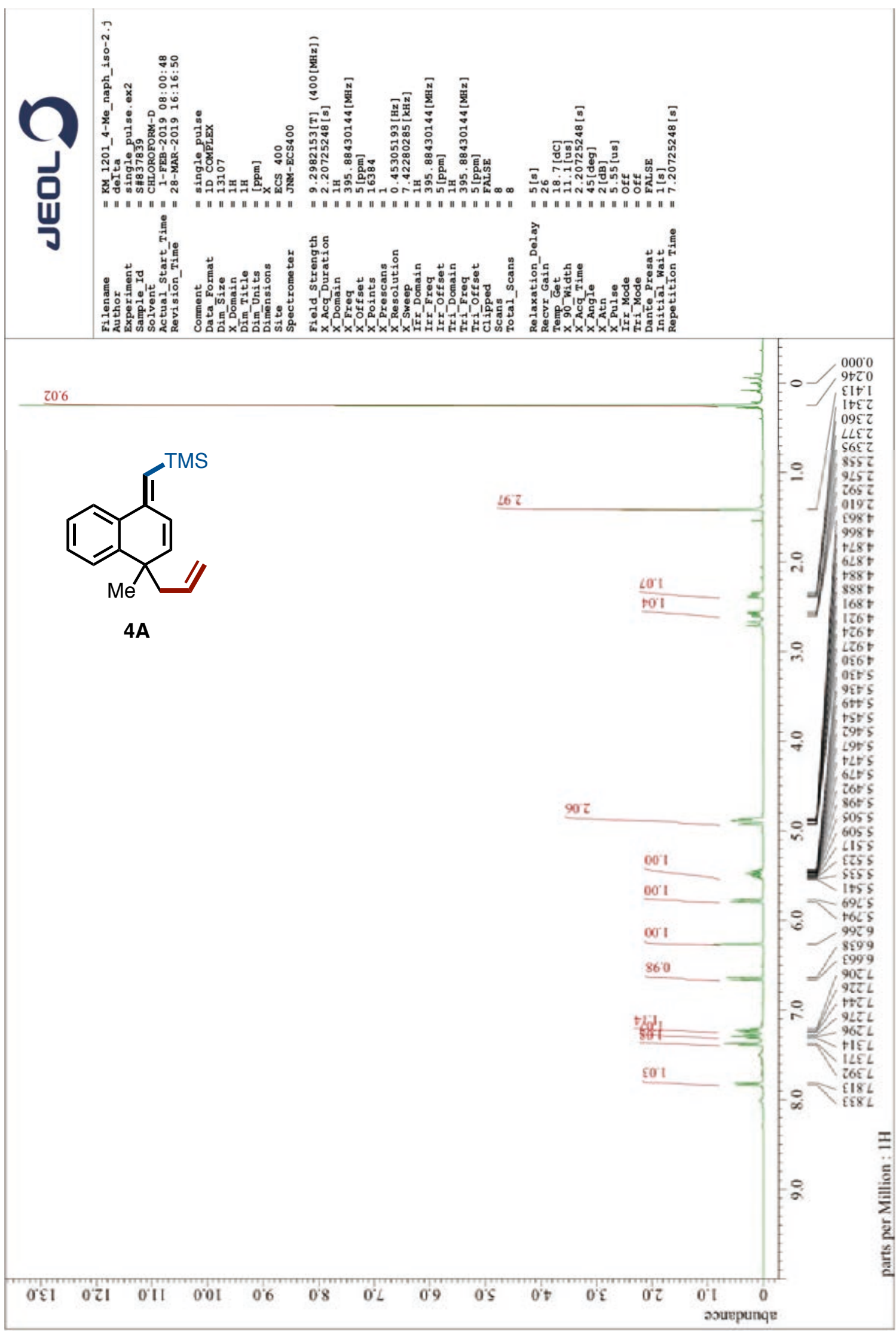


${ }^{13} \mathrm{C}$ NMR of $4 \mathrm{~A}\left(101 \mathrm{MHz}, \mathrm{CDCl}_{3}\right)$

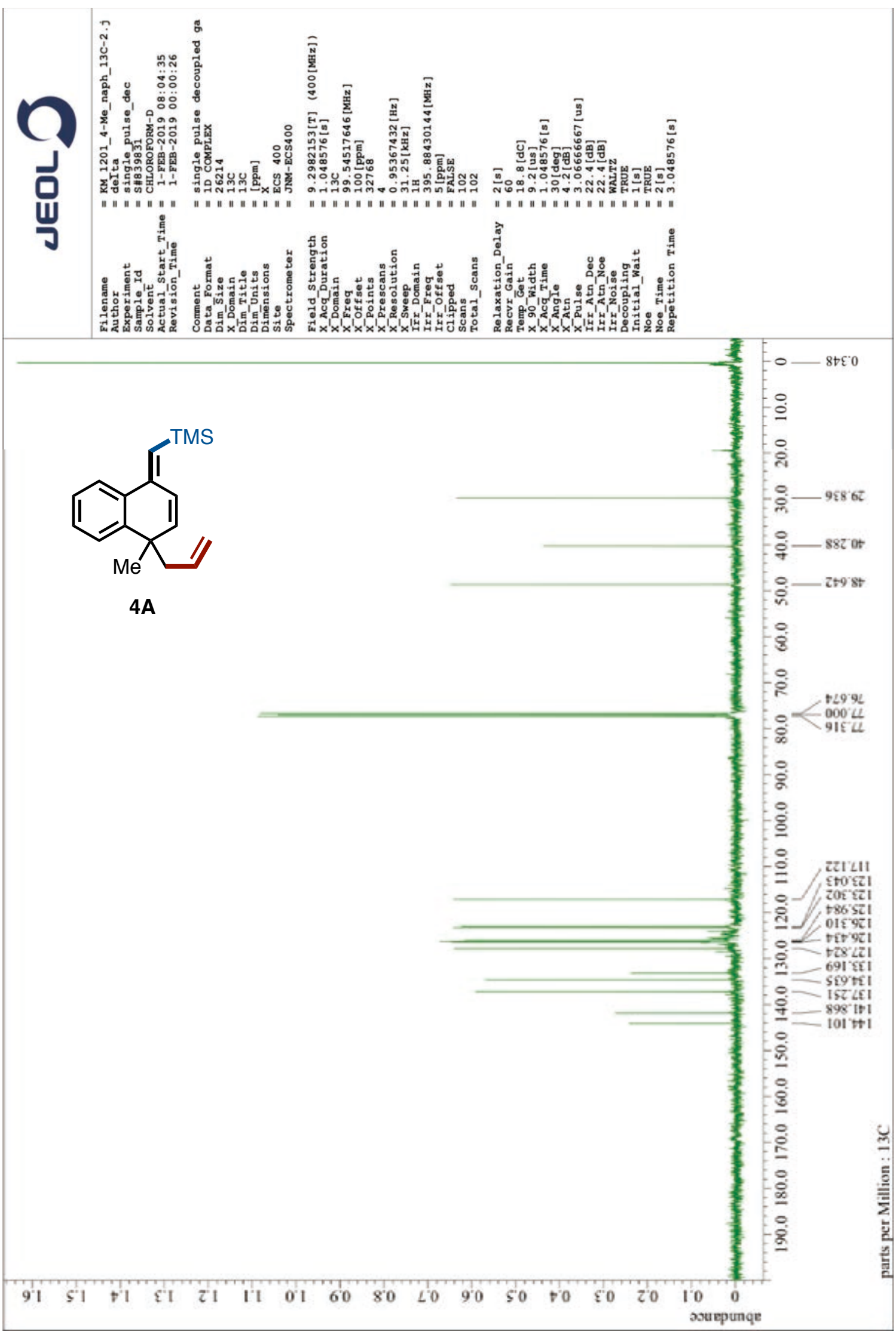


${ }^{1} \mathrm{H}$ NMR of $4 \mathbf{B}\left(400 \mathrm{MHz}, \mathrm{CDCl}_{3}\right)$

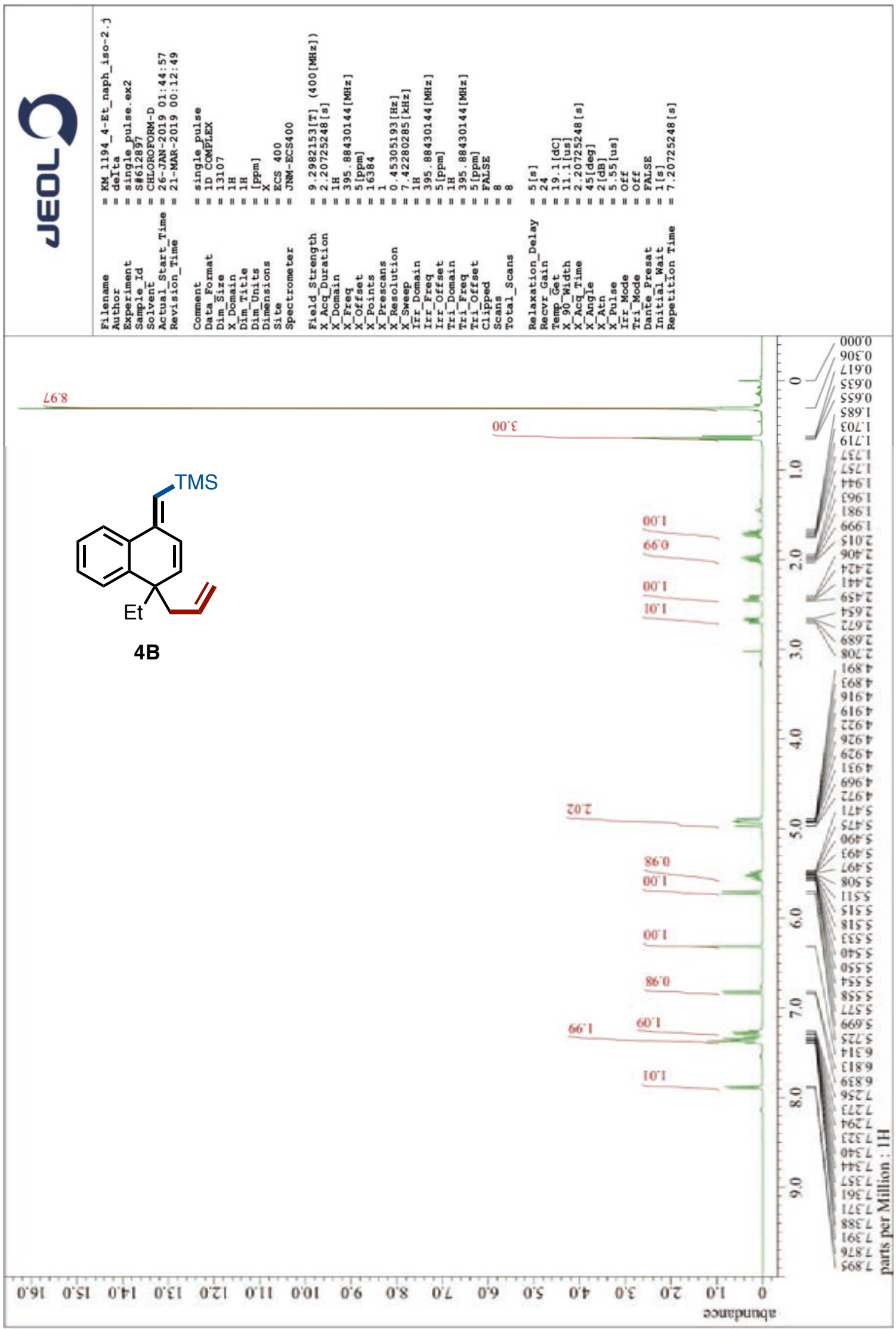


${ }^{13} \mathrm{C}$ NMR of $4 \mathrm{~B}\left(101 \mathrm{MHz}, \mathrm{CDCl}_{3}\right)$

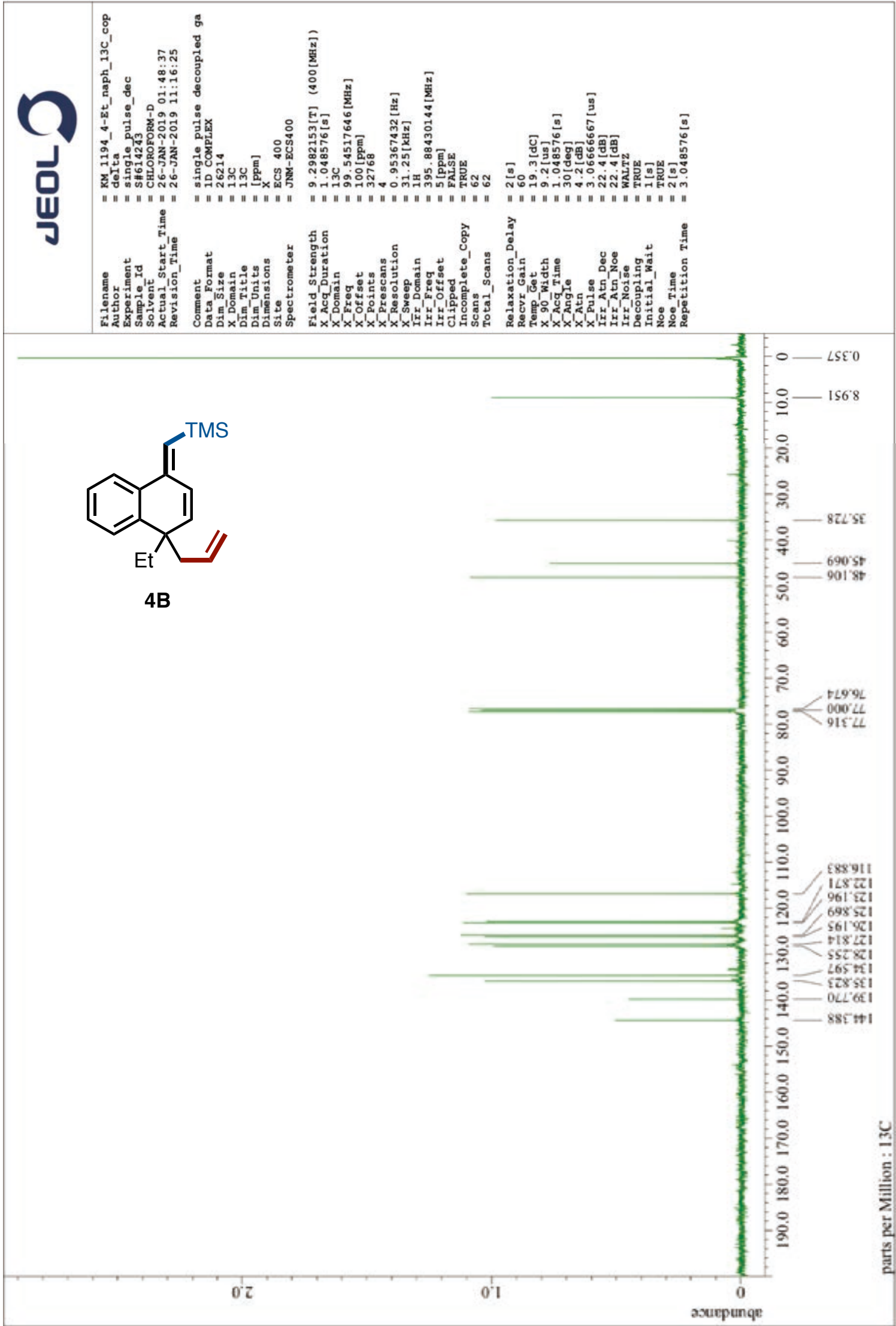


${ }^{1} \mathrm{H}$ NMR of 4C $\left(400 \mathrm{MHz}, \mathrm{CDCl}_{3}\right)$

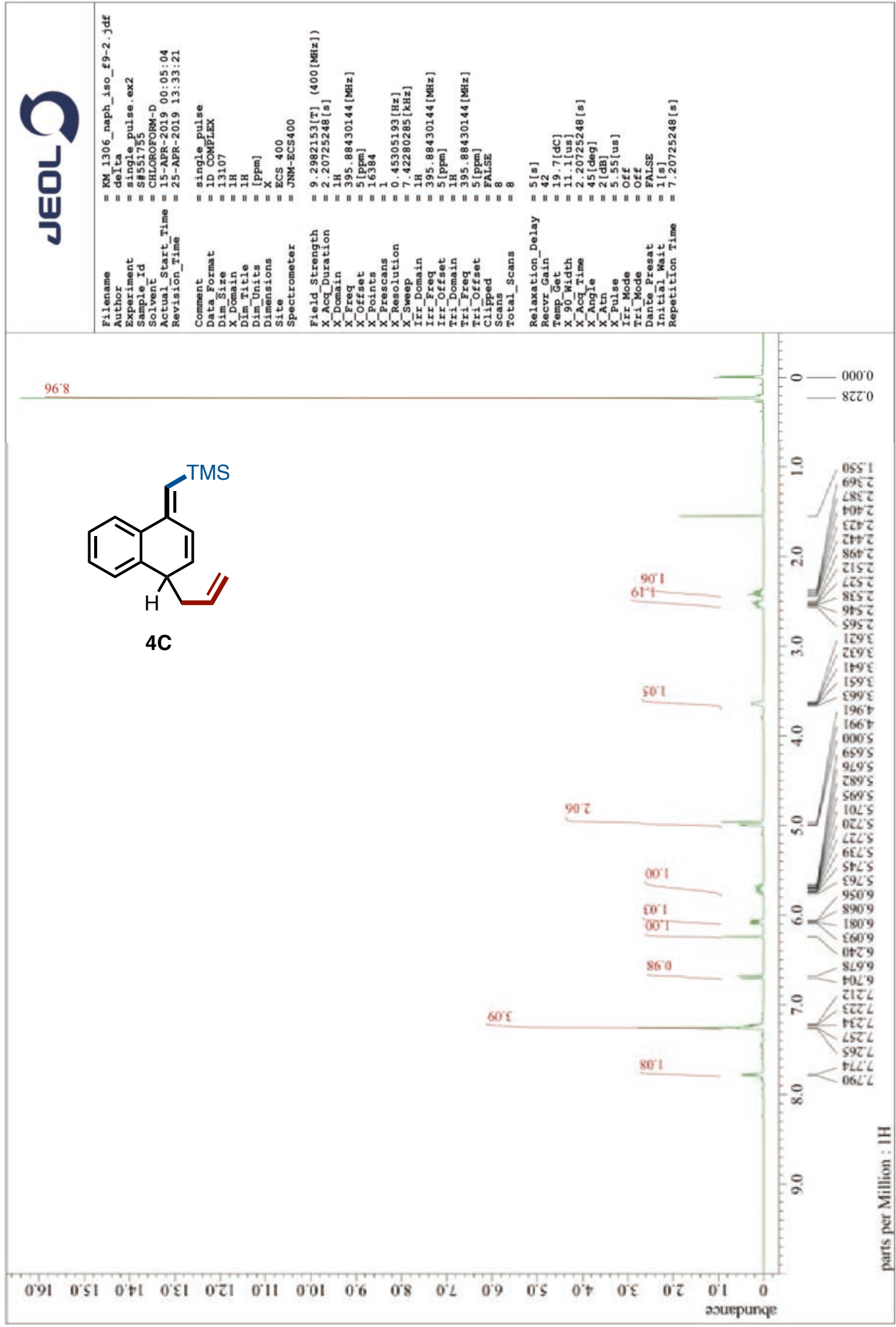


${ }^{13} \mathrm{C}$ NMR of $4 \mathrm{C}\left(101 \mathrm{MHz}, \mathrm{CDCl}_{3}\right)$

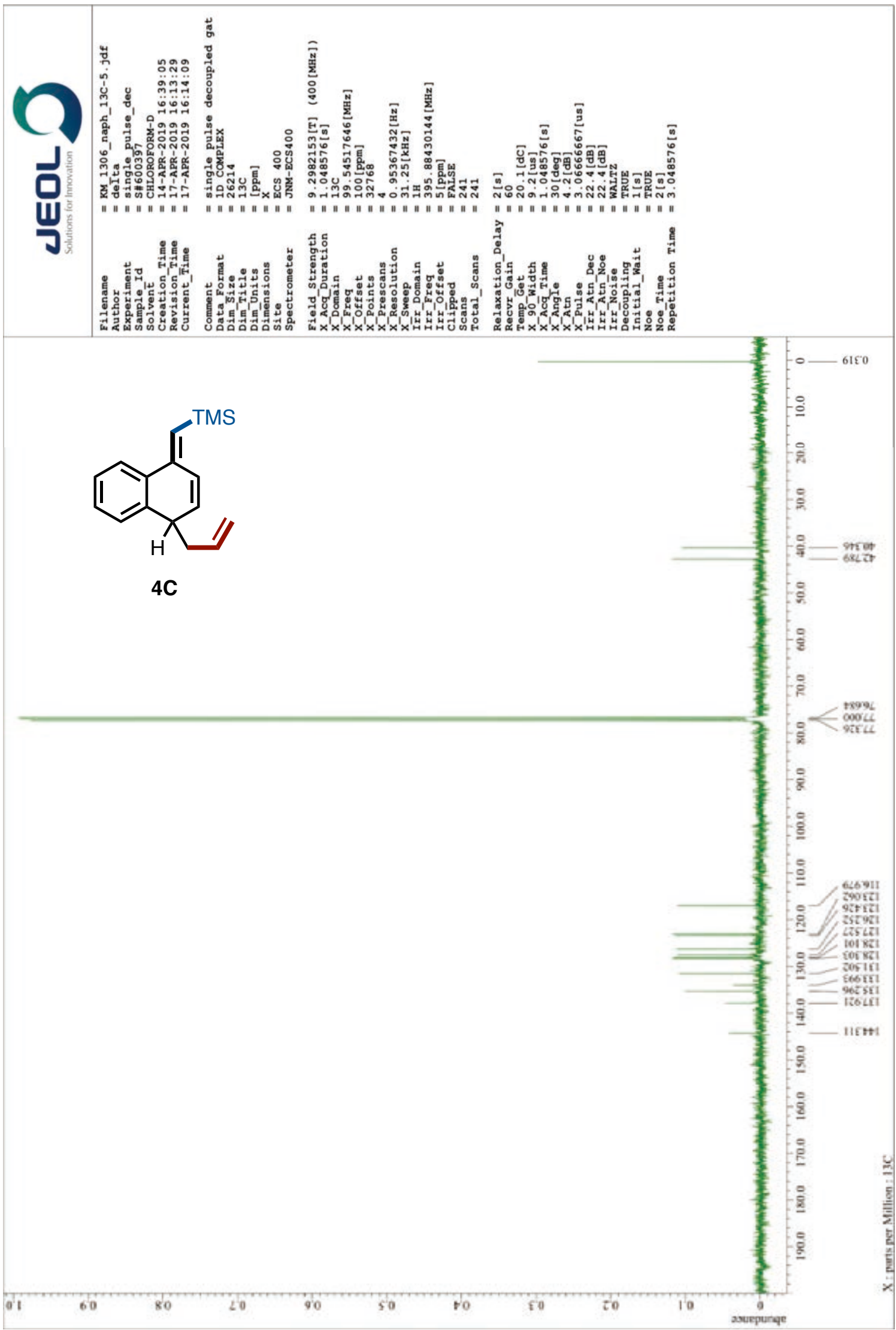


${ }^{1} \mathrm{H} \mathrm{NMR}$ of $4 \mathbf{D}\left(400 \mathrm{MHz}, \mathrm{CDCl}_{3}\right)$

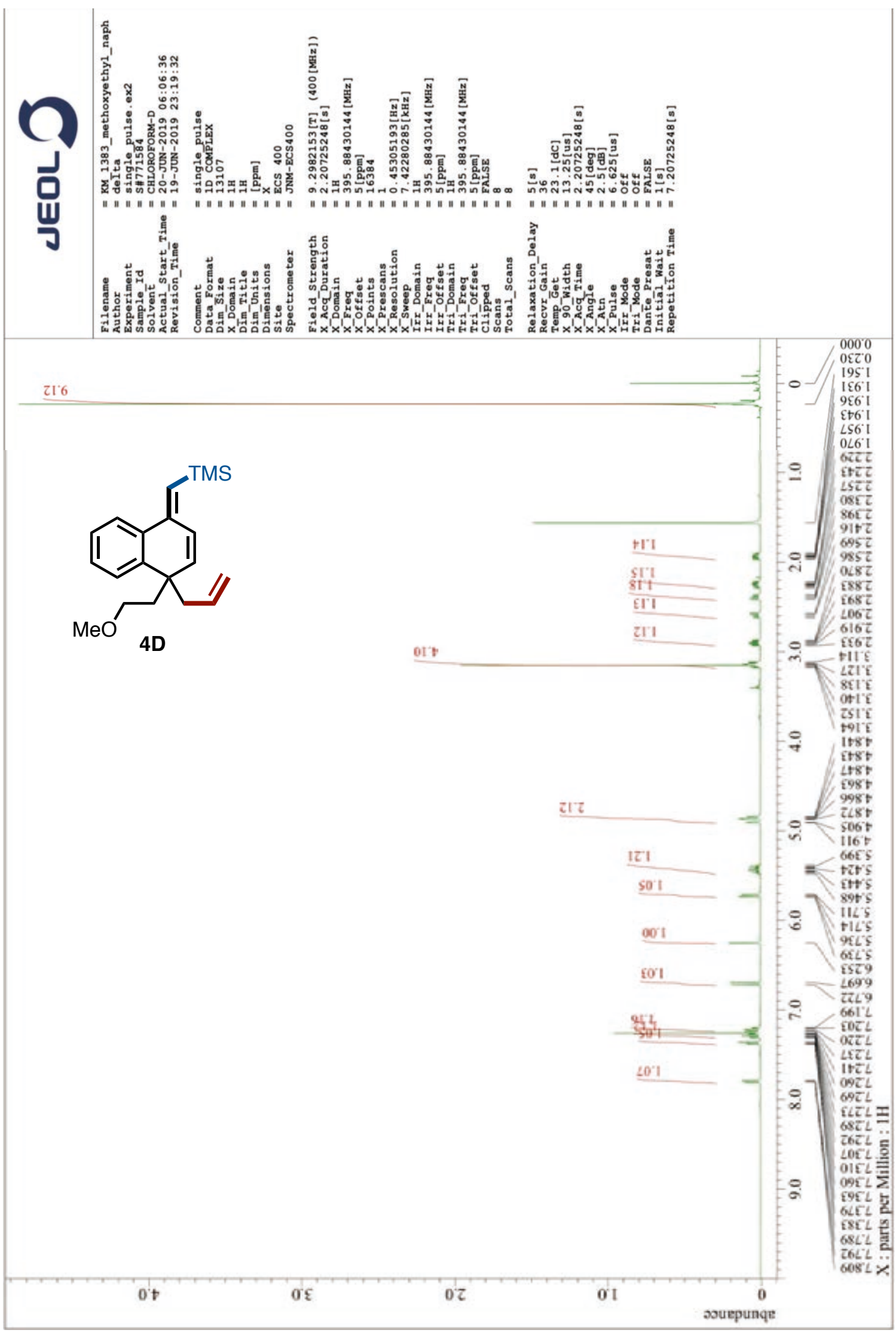


${ }^{13} \mathrm{C}$ NMR of $4 \mathbf{D}\left(101 \mathrm{MHz}, \mathrm{CDCl}_{3}\right)$

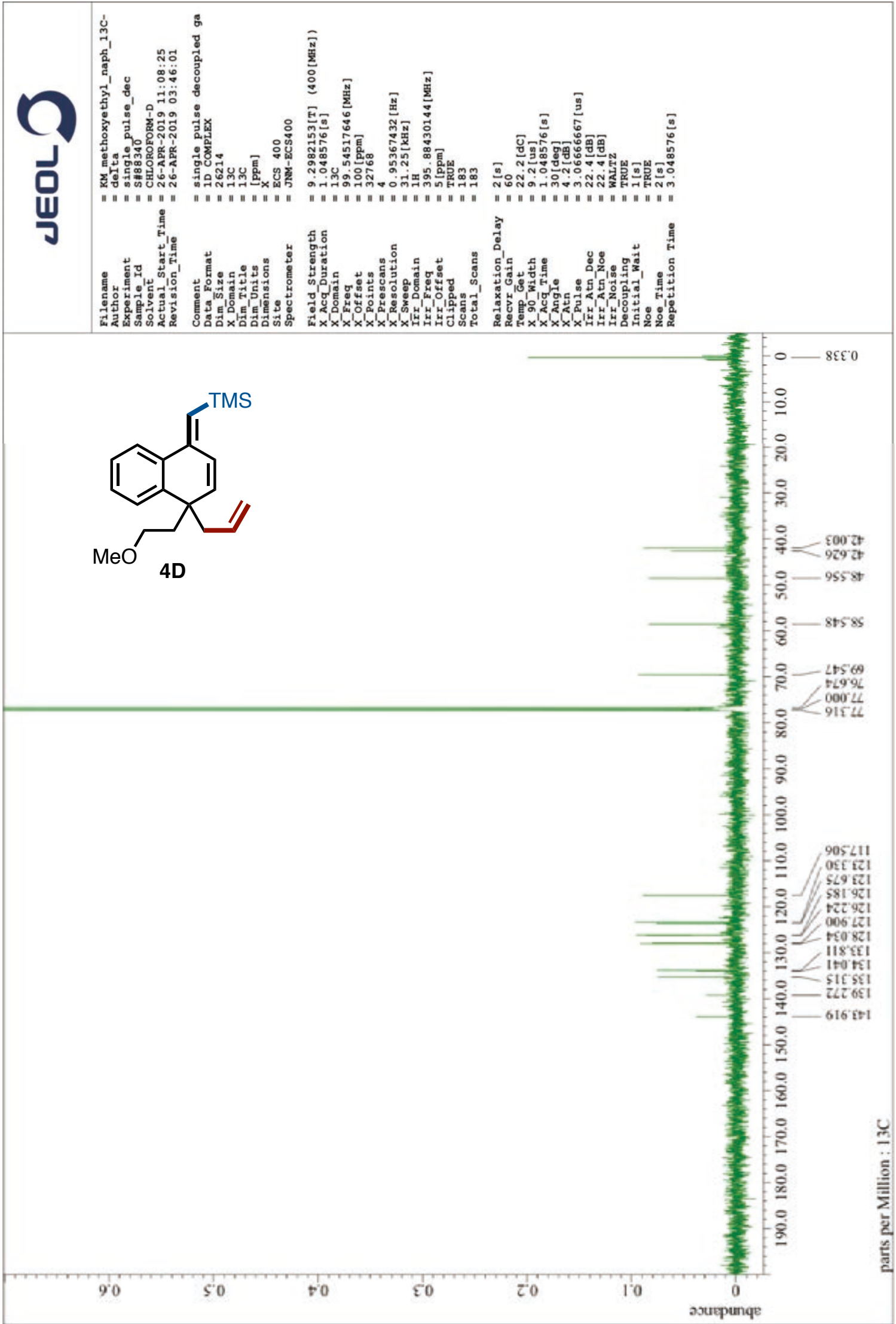


${ }^{1} \mathrm{H}$ NMR of $4 \mathbf{E}\left(400 \mathrm{MHz}, \mathrm{CDCl}_{3}\right)$

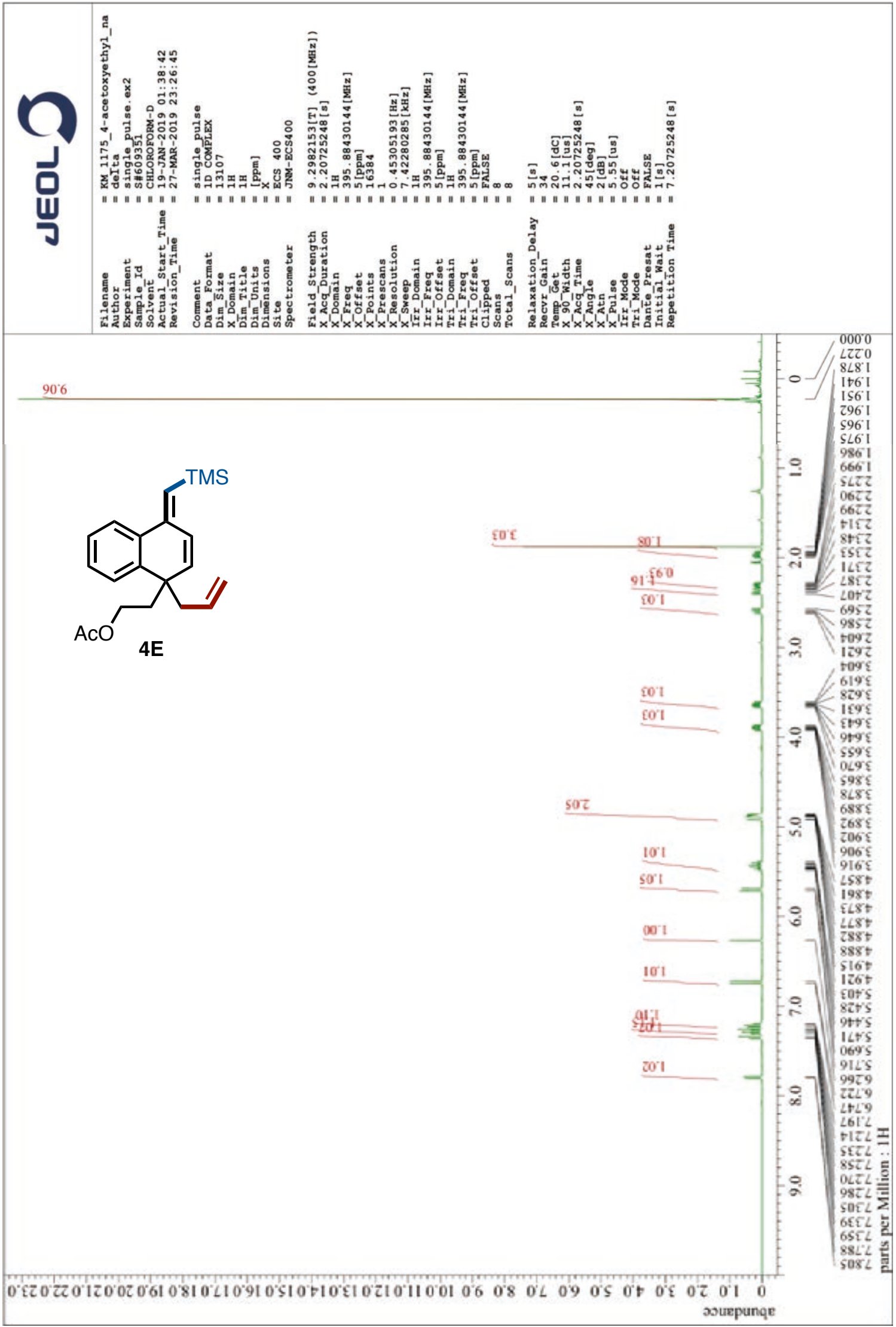


${ }^{13} \mathrm{C}$ NMR of $4 \mathbf{E}\left(101 \mathrm{MHz}, \mathrm{CDCl}_{3}\right)$

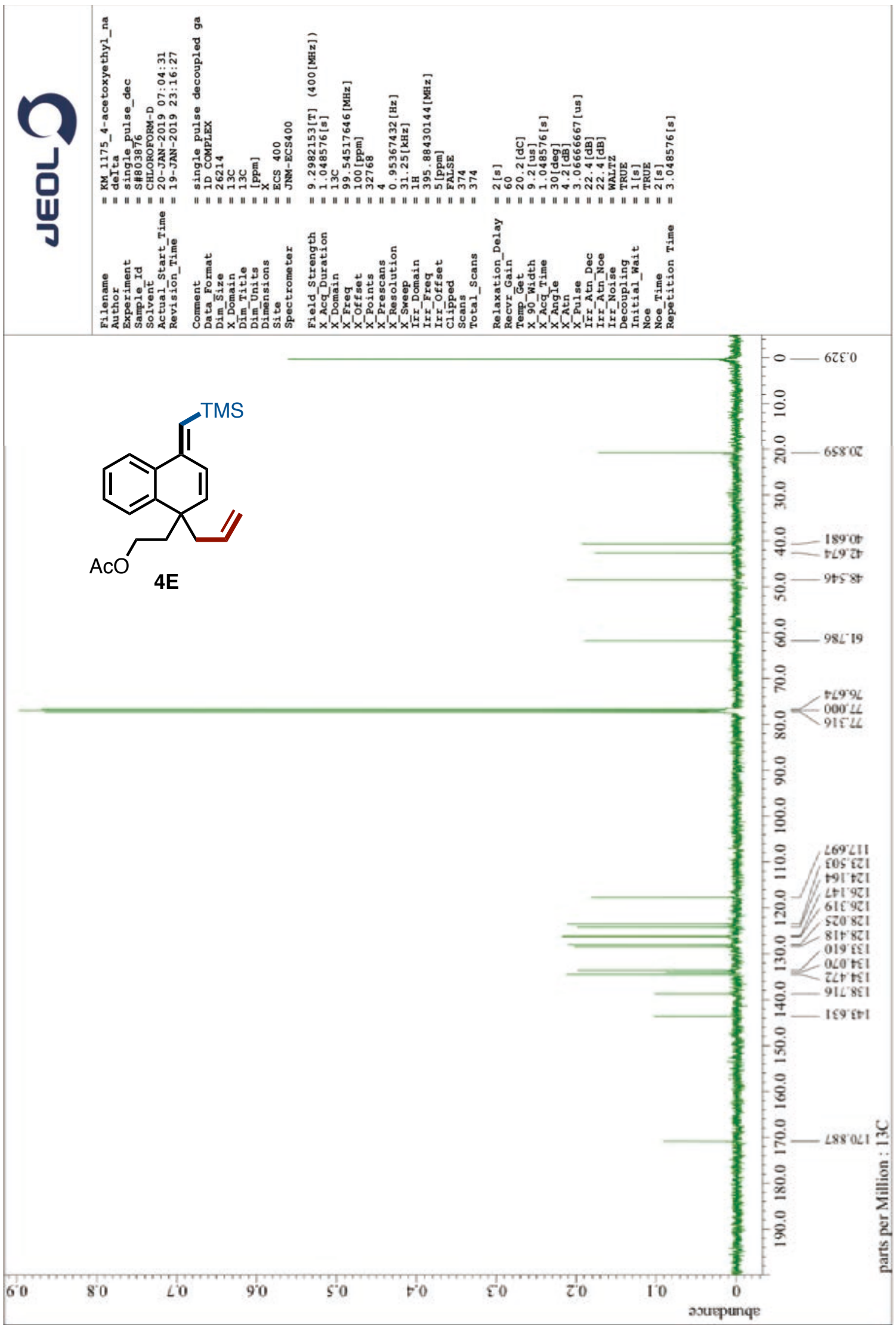


${ }^{1} \mathrm{H}$ NMR of $4 \mathbf{F}\left(400 \mathrm{MHz}, \mathrm{CDCl}_{3}\right)$

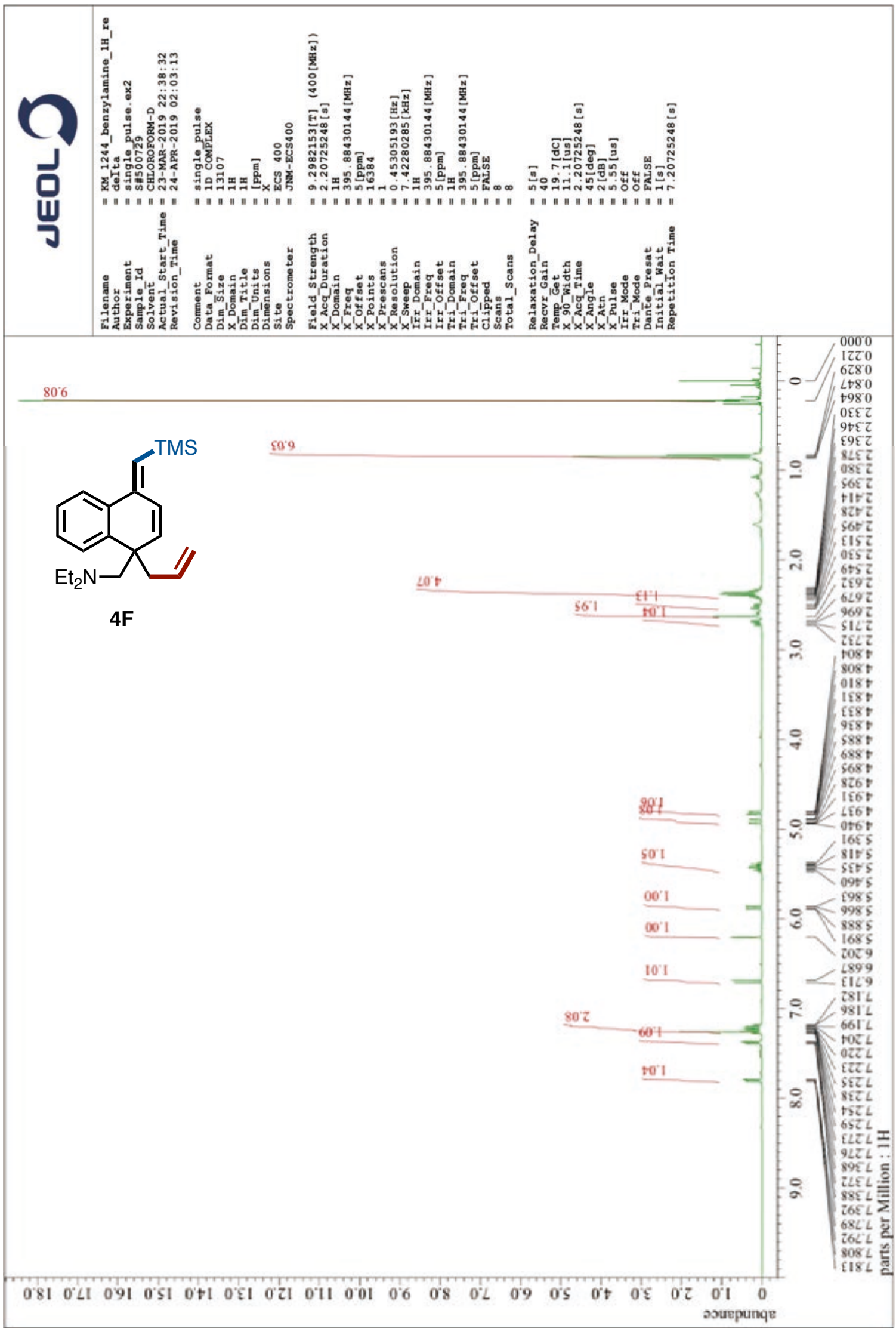


${ }^{13} \mathrm{C}$ NMR of 4 F $\left(101 \mathrm{MHz}, \mathrm{CDCl}_{3}\right)$

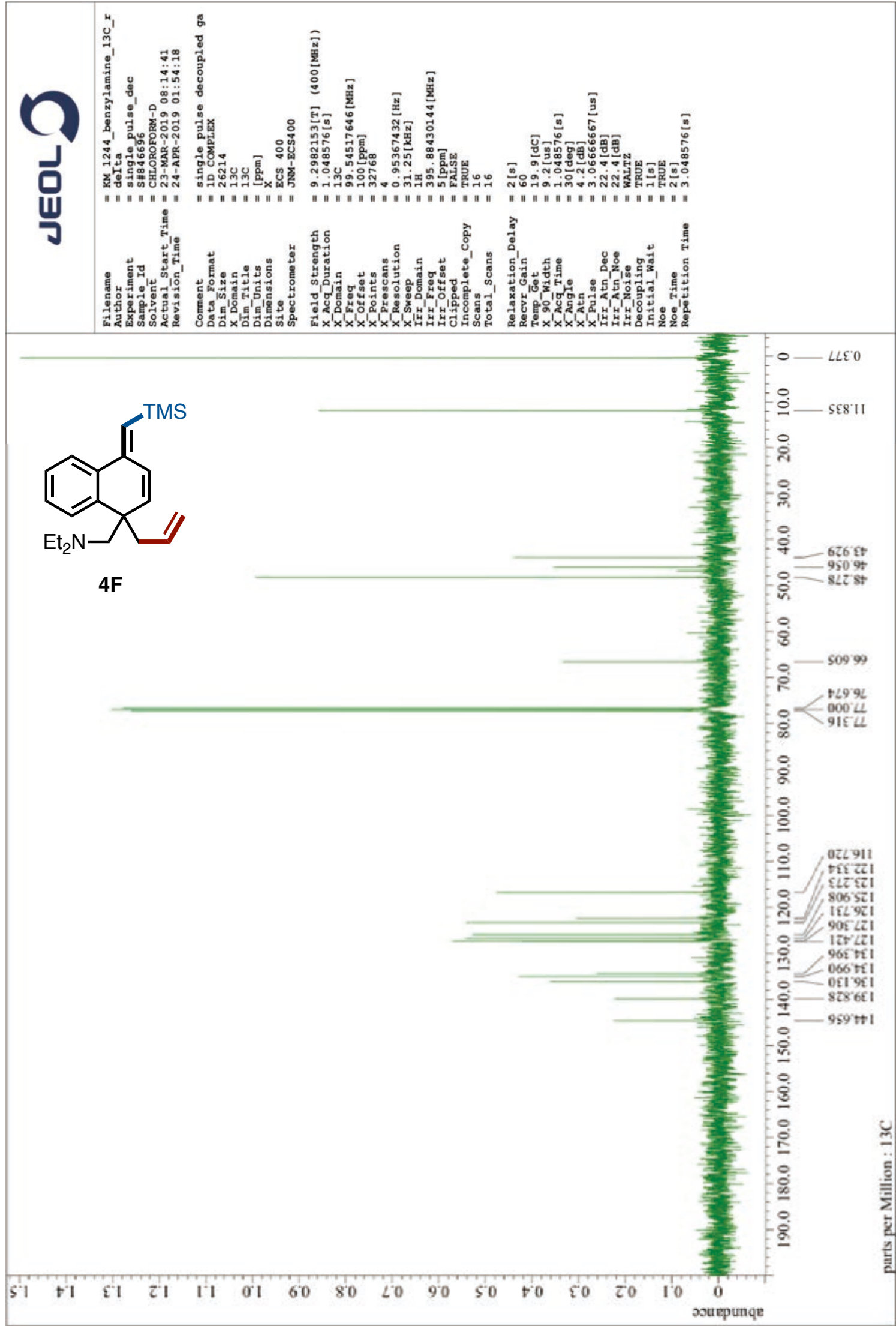


${ }^{1} \mathrm{H}$ NMR of $\mathbf{4 G}\left(400 \mathrm{MHz}, \mathrm{CDCl}_{3}\right)$

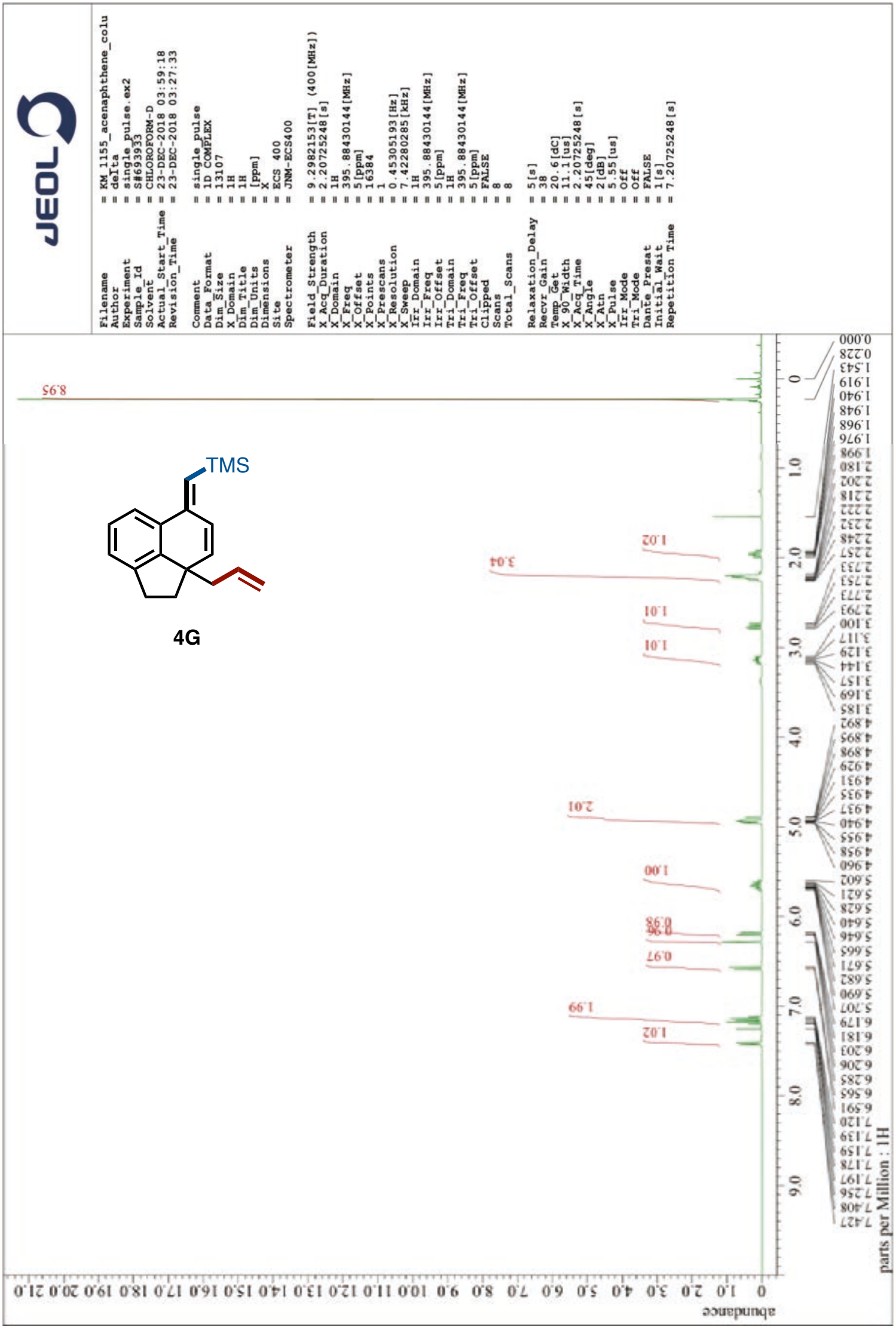


${ }^{13} \mathrm{C}$ NMR of $\mathbf{4 G}\left(101 \mathrm{MHz}, \mathrm{CDCl}_{3}\right)$

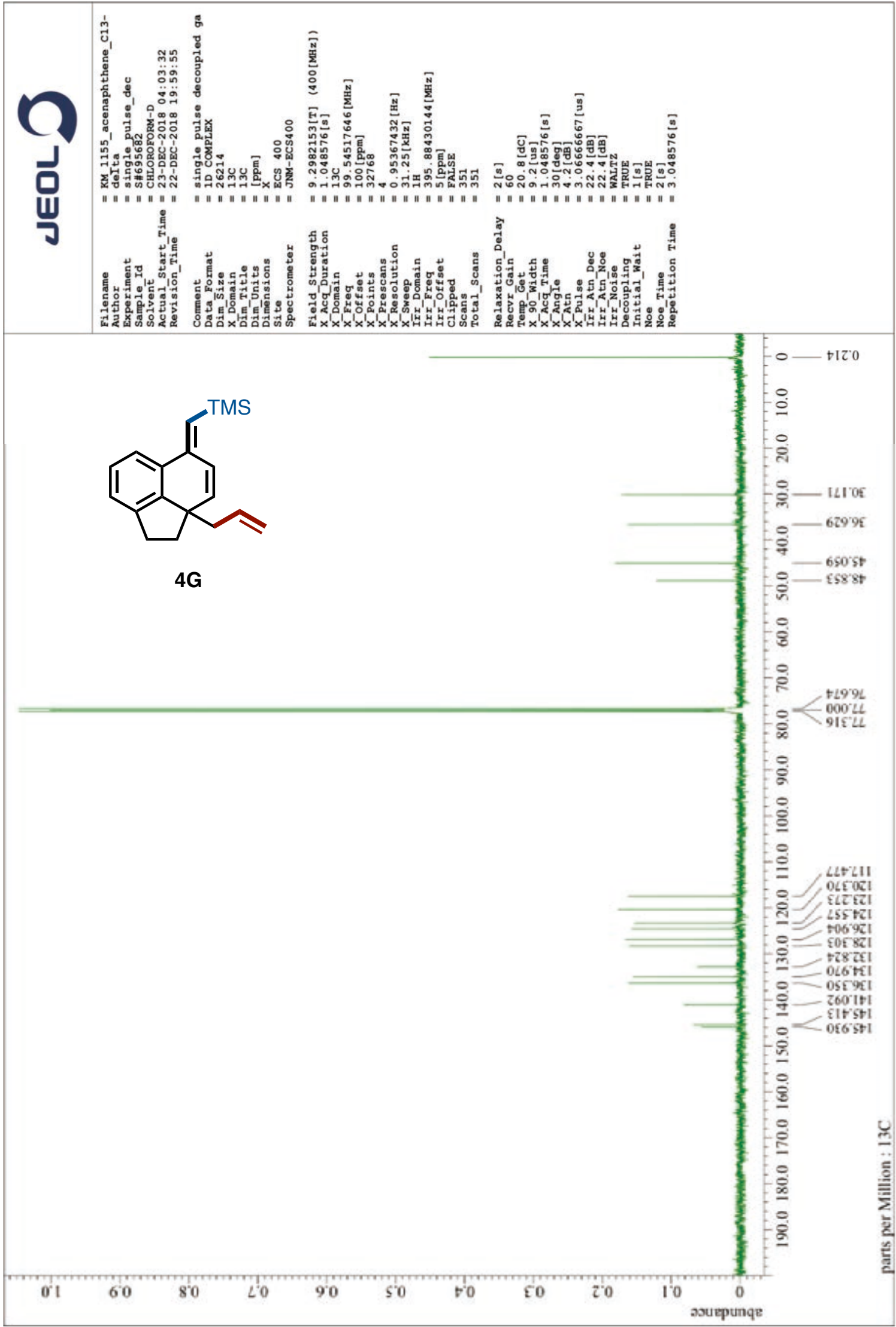


${ }^{1} \mathrm{H}$ NMR of $\mathbf{4 H}\left(400 \mathrm{MHz}, \mathrm{CDCl}_{3}\right)$

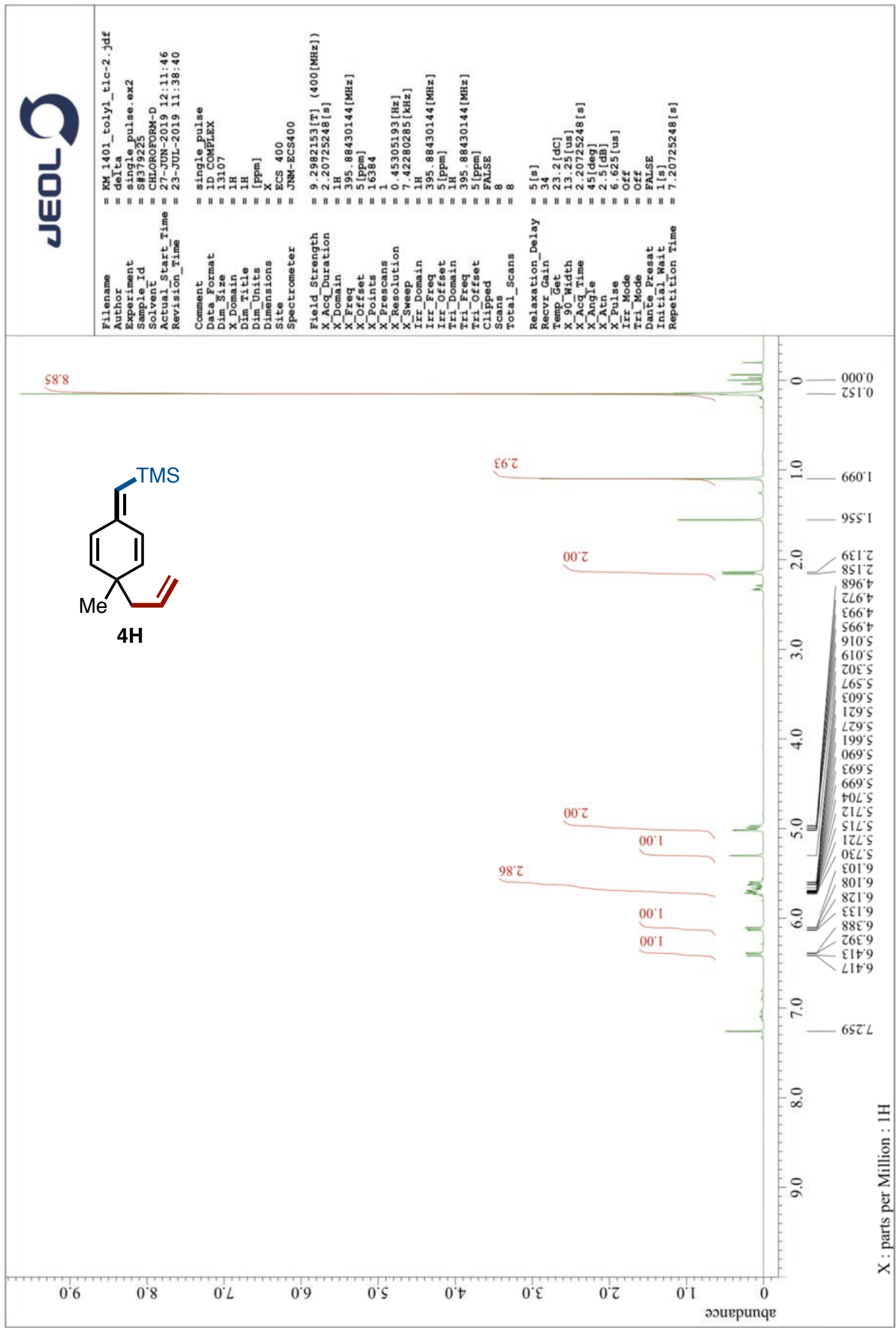


${ }^{13} \mathrm{C}$ NMR of $4 \mathbf{H}\left(101 \mathrm{MHz}, \mathrm{CDCl}_{3}\right)$

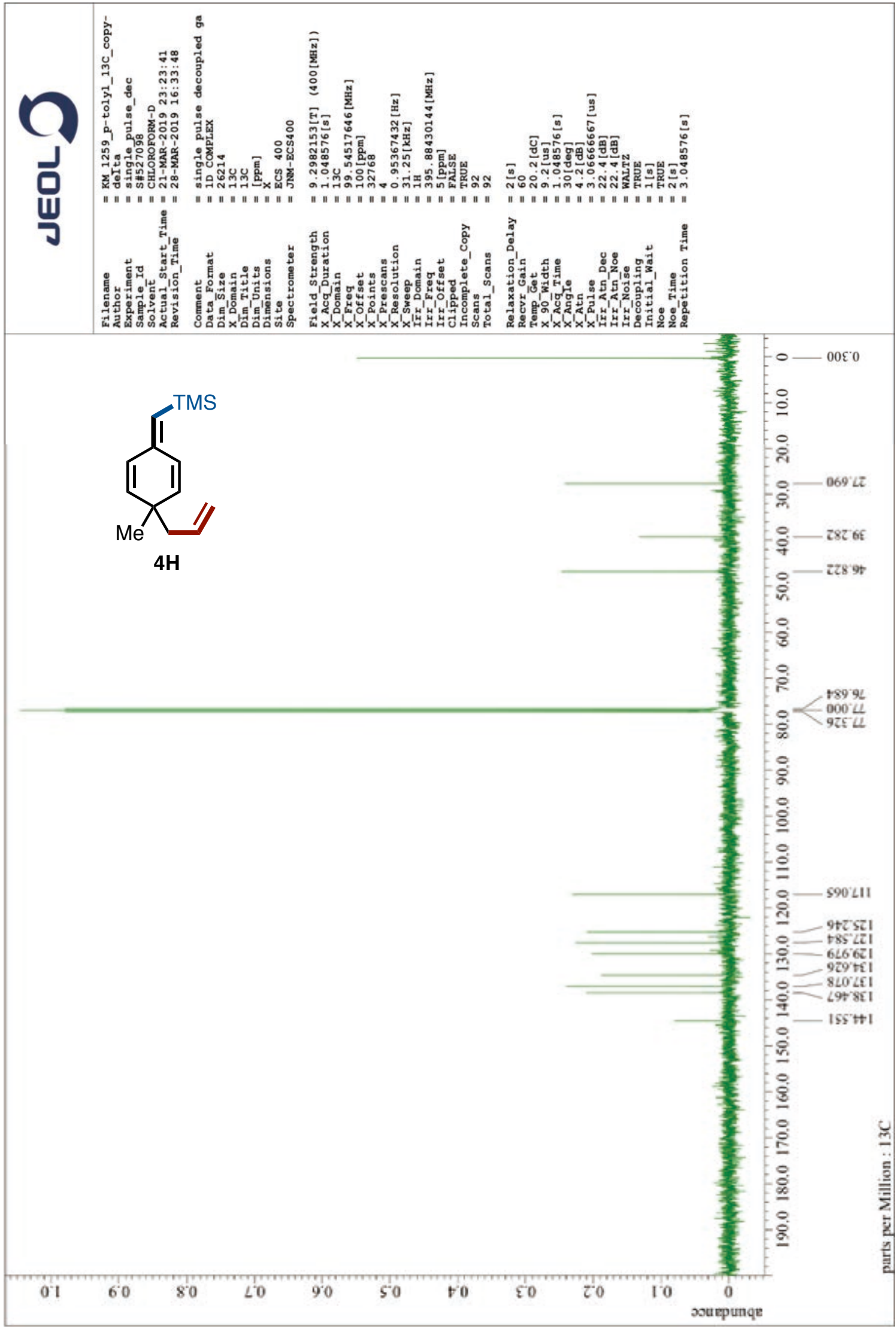


${ }^{1} \mathrm{H}$ NMR of $4 \mathbf{I}\left(400 \mathrm{MHz}, \mathrm{CDCl}_{3}\right)$

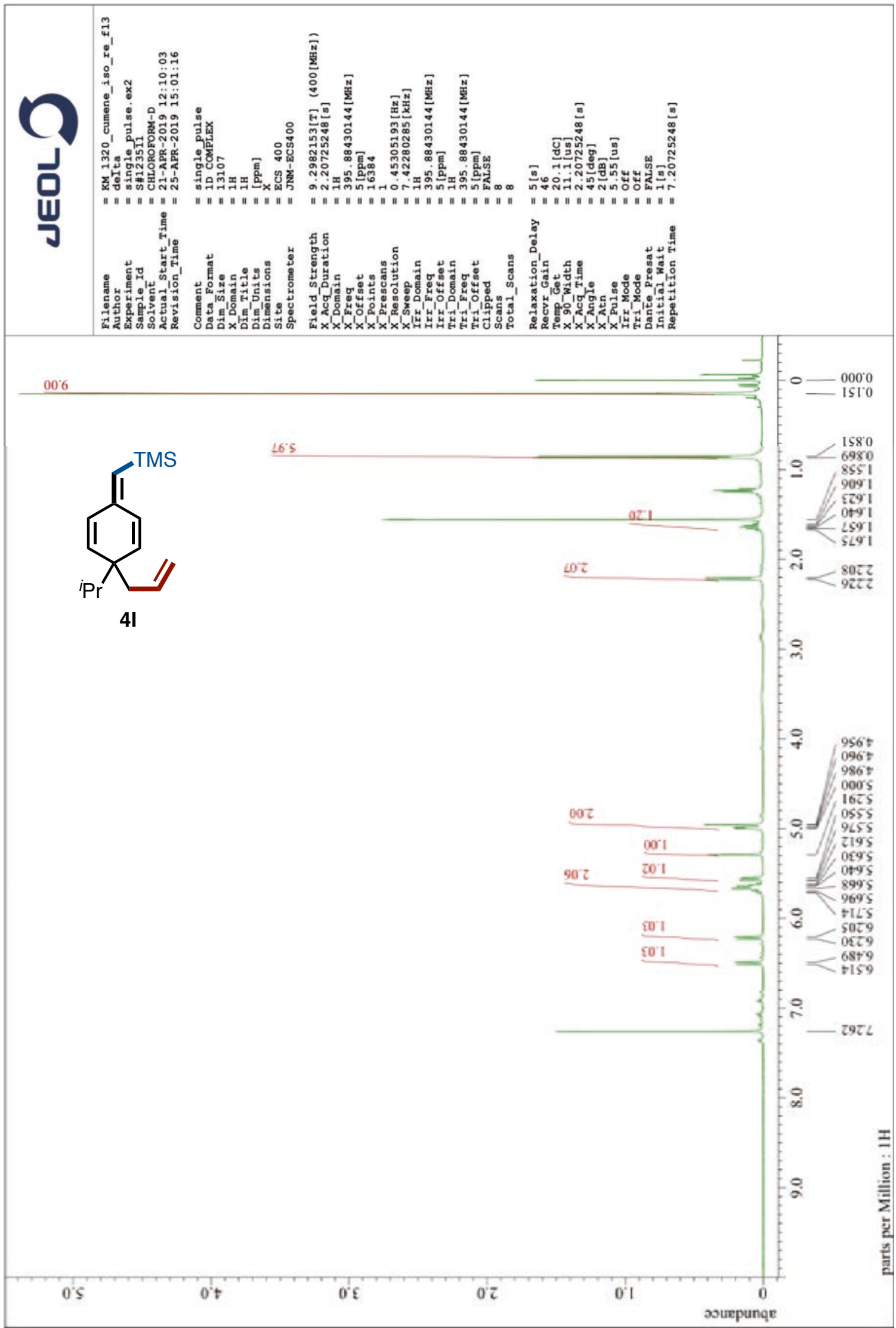


${ }^{13} \mathrm{C}$ NMR of 4 I $\left(101 \mathrm{MHz}, \mathrm{CDCl}_{3}\right)$

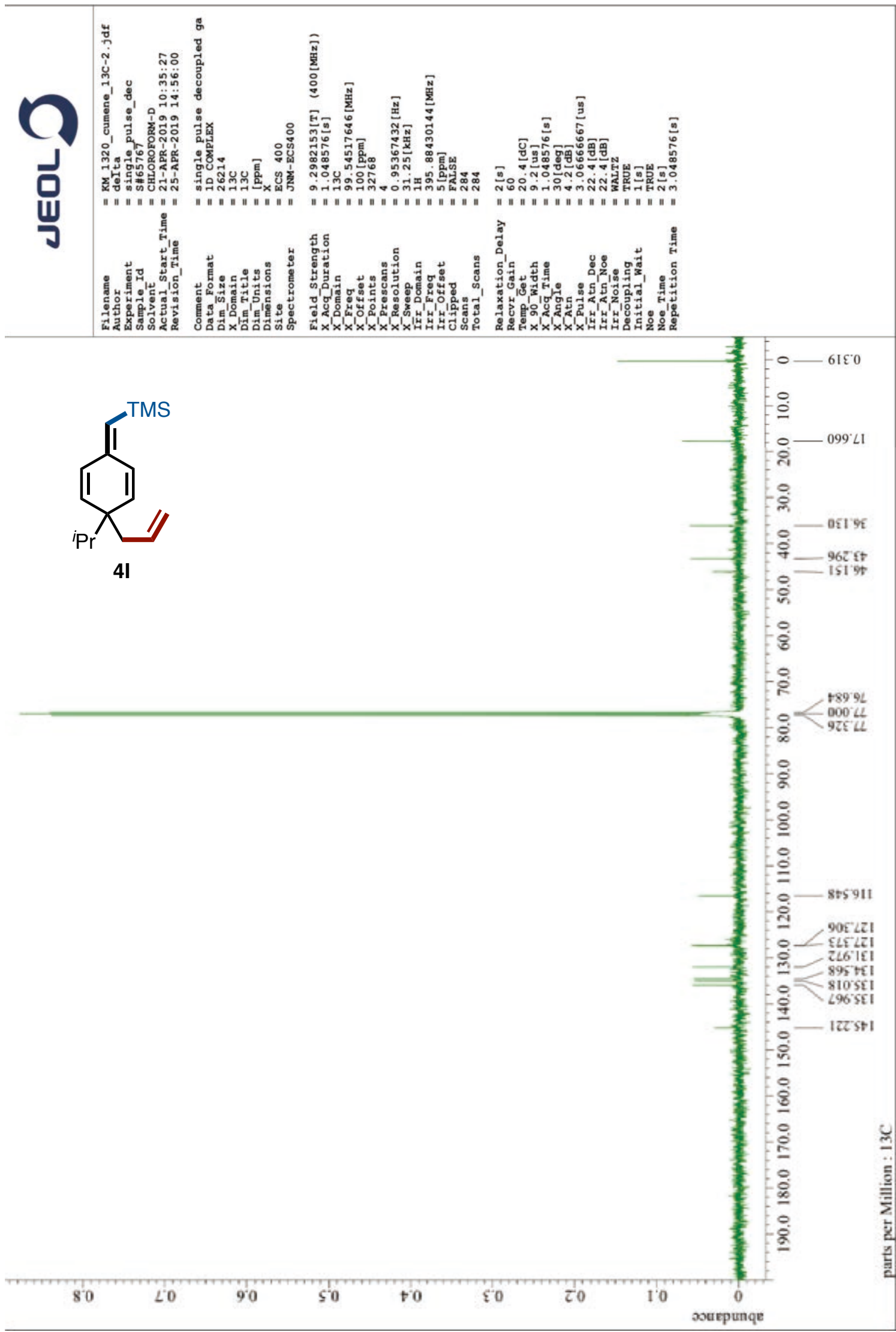


${ }^{1} \mathrm{H}$ NMR of $4 \mathbf{J}\left(400 \mathrm{MHz}, \mathrm{CDCl}_{3}\right)$

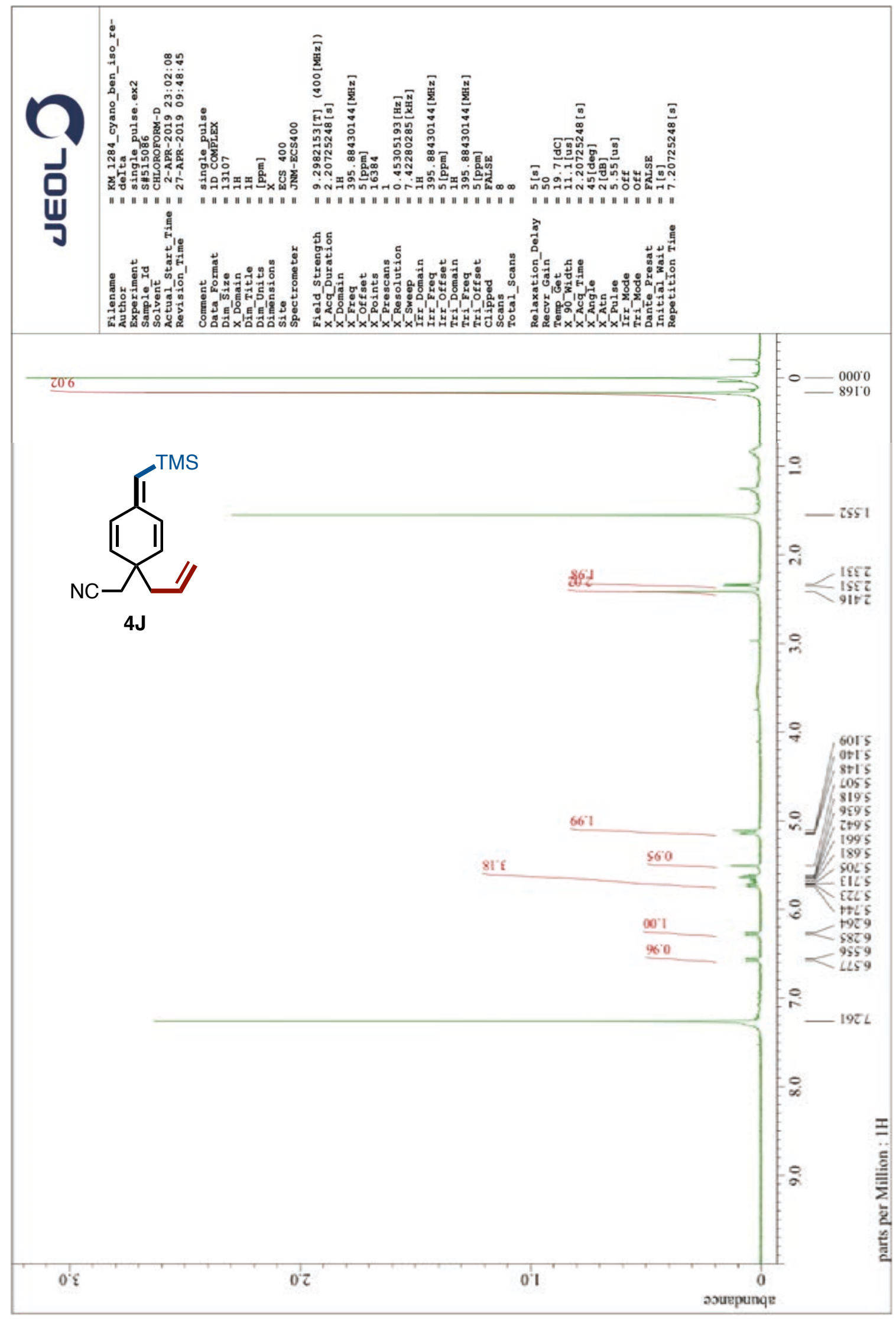


${ }^{13} \mathrm{C}$ NMR of $4 \mathbf{J}\left(101 \mathrm{MHz}, \mathrm{CDCl}_{3}\right)$

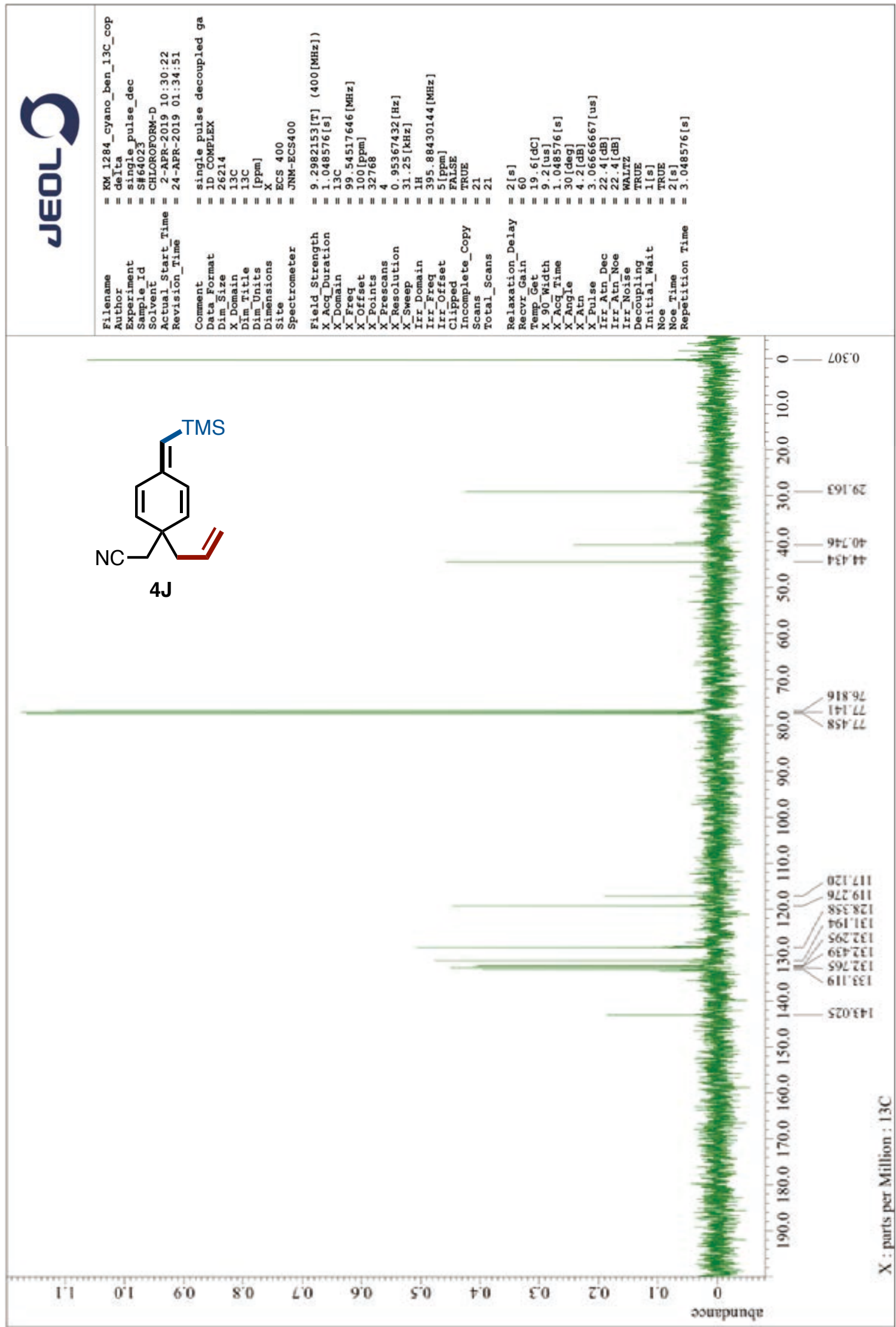


${ }^{1} \mathrm{H} \mathrm{NMR}$ of $\mathbf{4 K}\left(400 \mathrm{MHz}, \mathrm{CDCl}_{3}\right)$

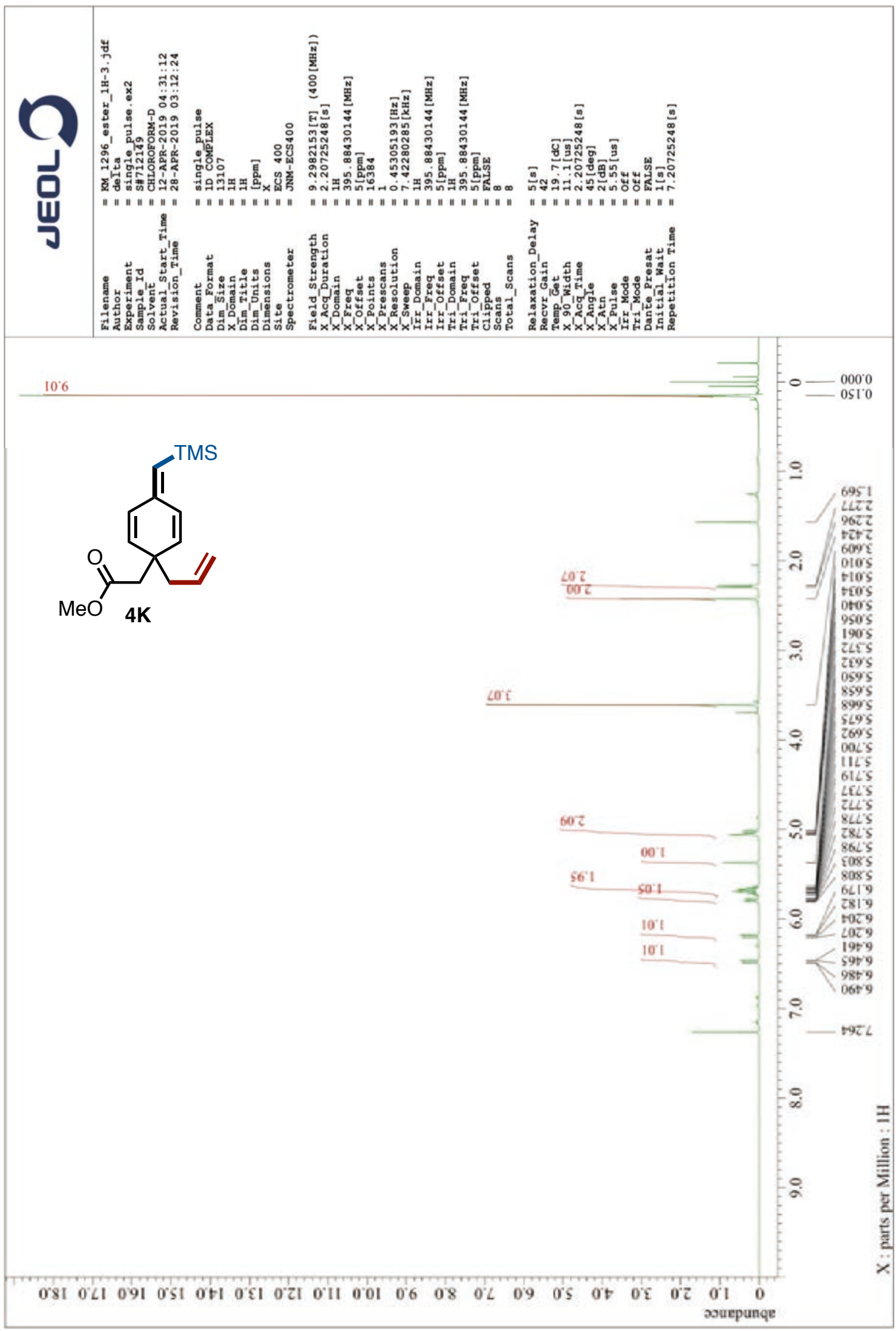


${ }^{13} \mathrm{C}$ NMR of $4 \mathbf{K}\left(101 \mathrm{MHz}, \mathrm{CDCl}_{3}\right)$

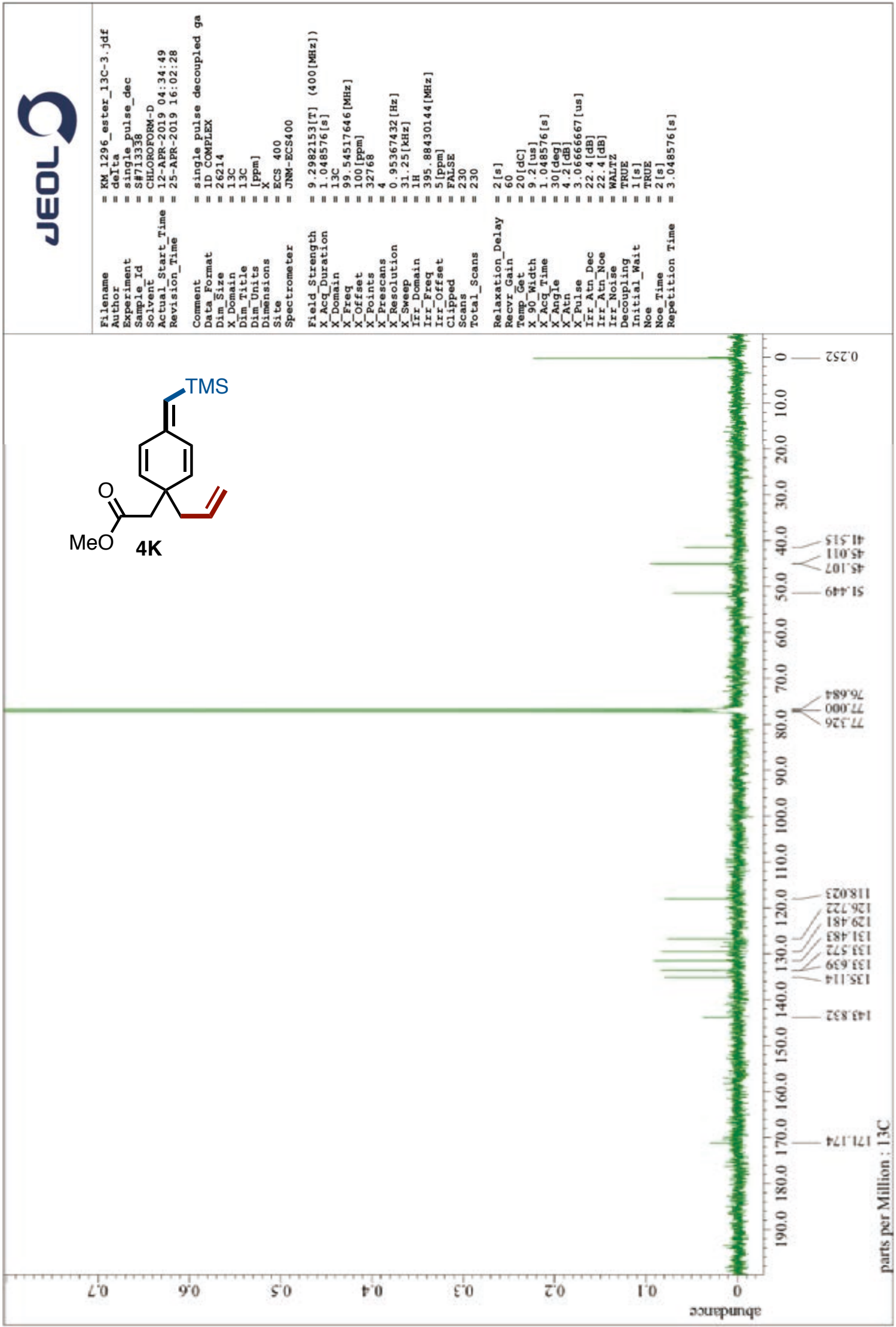


${ }^{1} \mathrm{H}$ NMR of $\mathbf{4} \mathbf{L}\left(400 \mathrm{MHz}, \mathrm{CDCl}_{3}\right)$

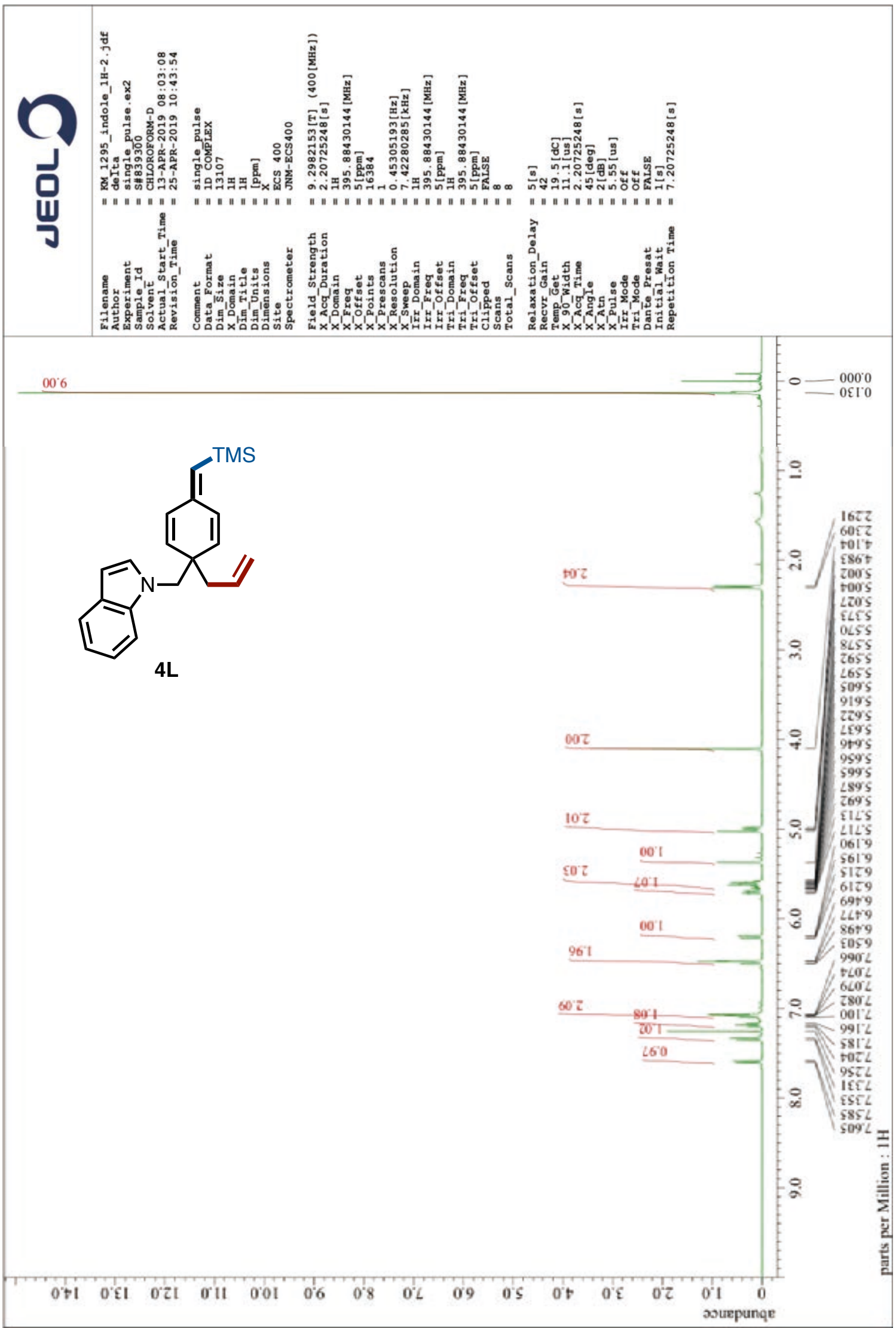


${ }^{13} \mathrm{C}$ NMR of $4 \mathbf{L}\left(101 \mathrm{MHz}, \mathrm{CDCl}_{3}\right)$

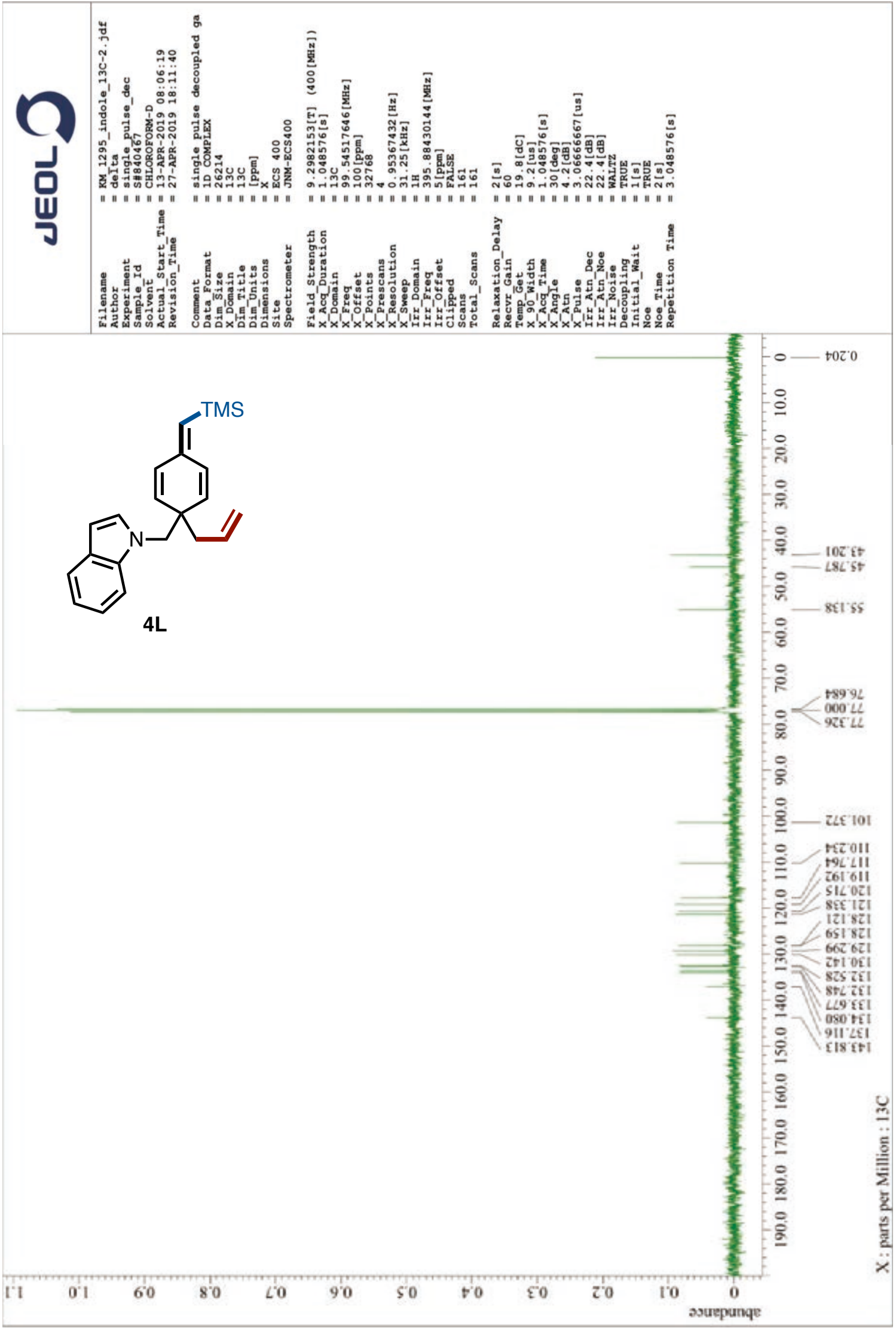


${ }^{1} \mathrm{H}$ NMR of $4 \mathbf{M}\left(400 \mathrm{MHz}, \mathrm{CDCl}_{3}\right)$

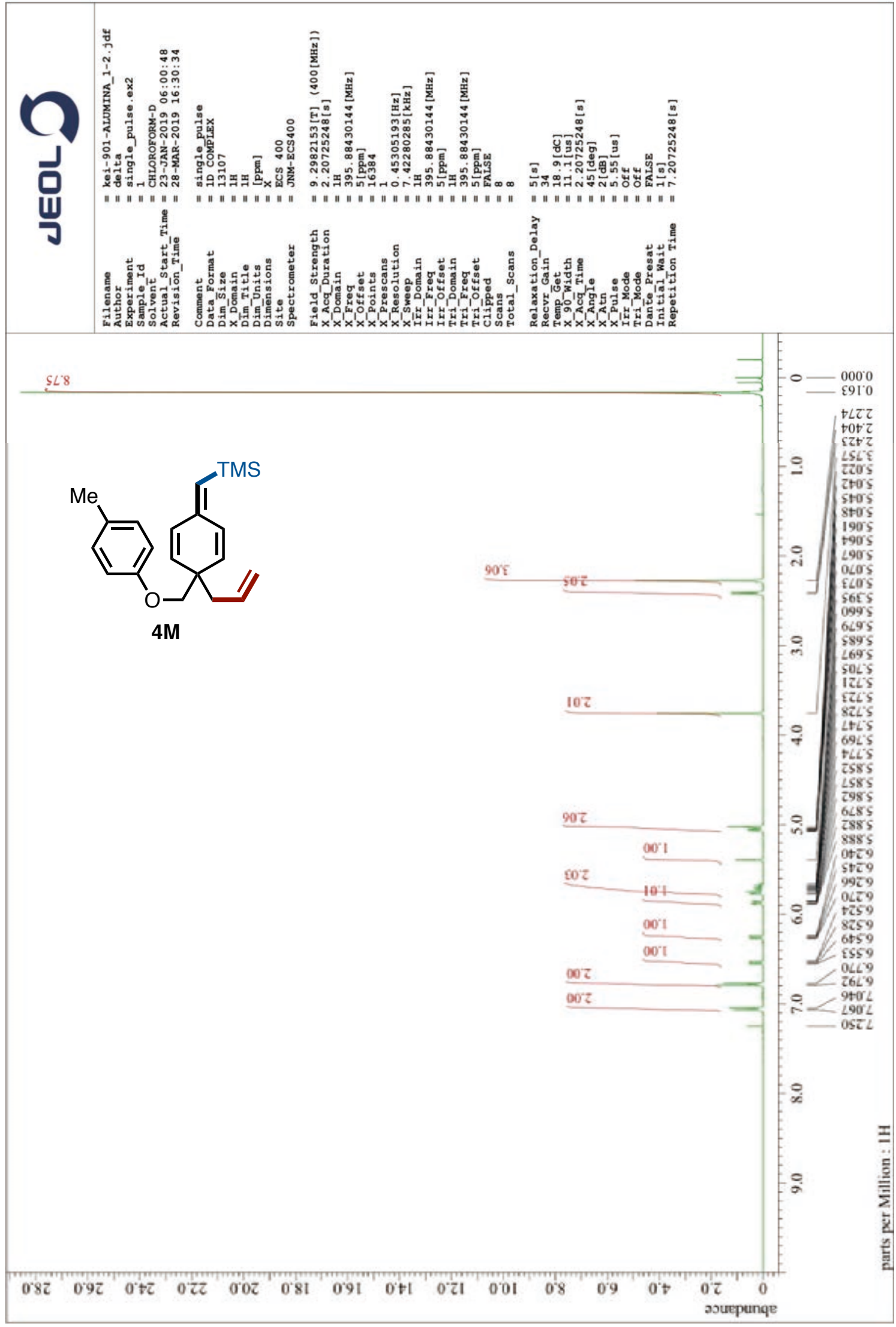


${ }^{13} \mathrm{C}$ NMR of $4 \mathbf{M}\left(101 \mathrm{MHz}, \mathrm{CDCl}_{3}\right)$

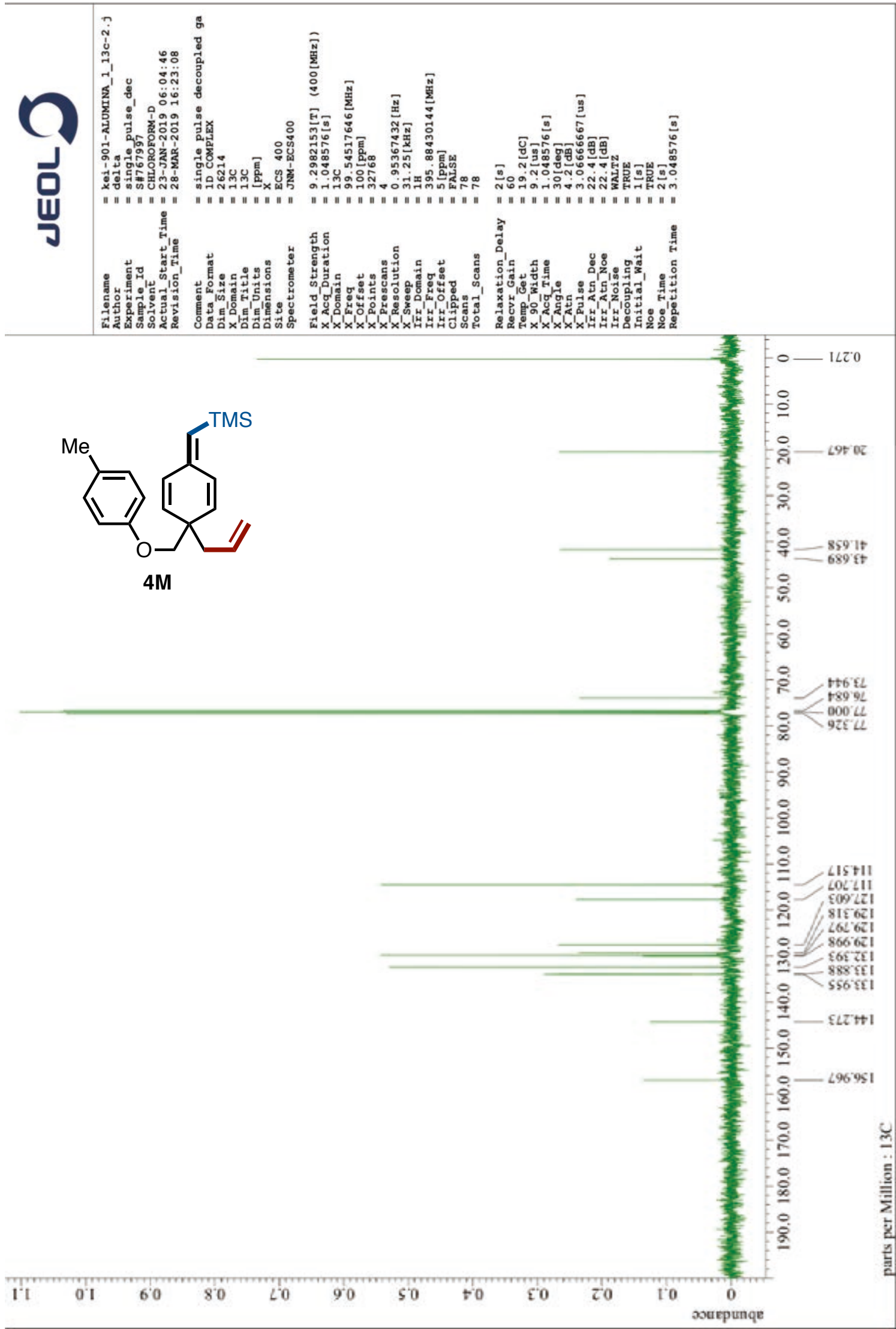


${ }^{1} \mathrm{H}$ NMR of $\mathbf{4 N}\left(400 \mathrm{MHz}, \mathrm{CDCl}_{3}\right)$

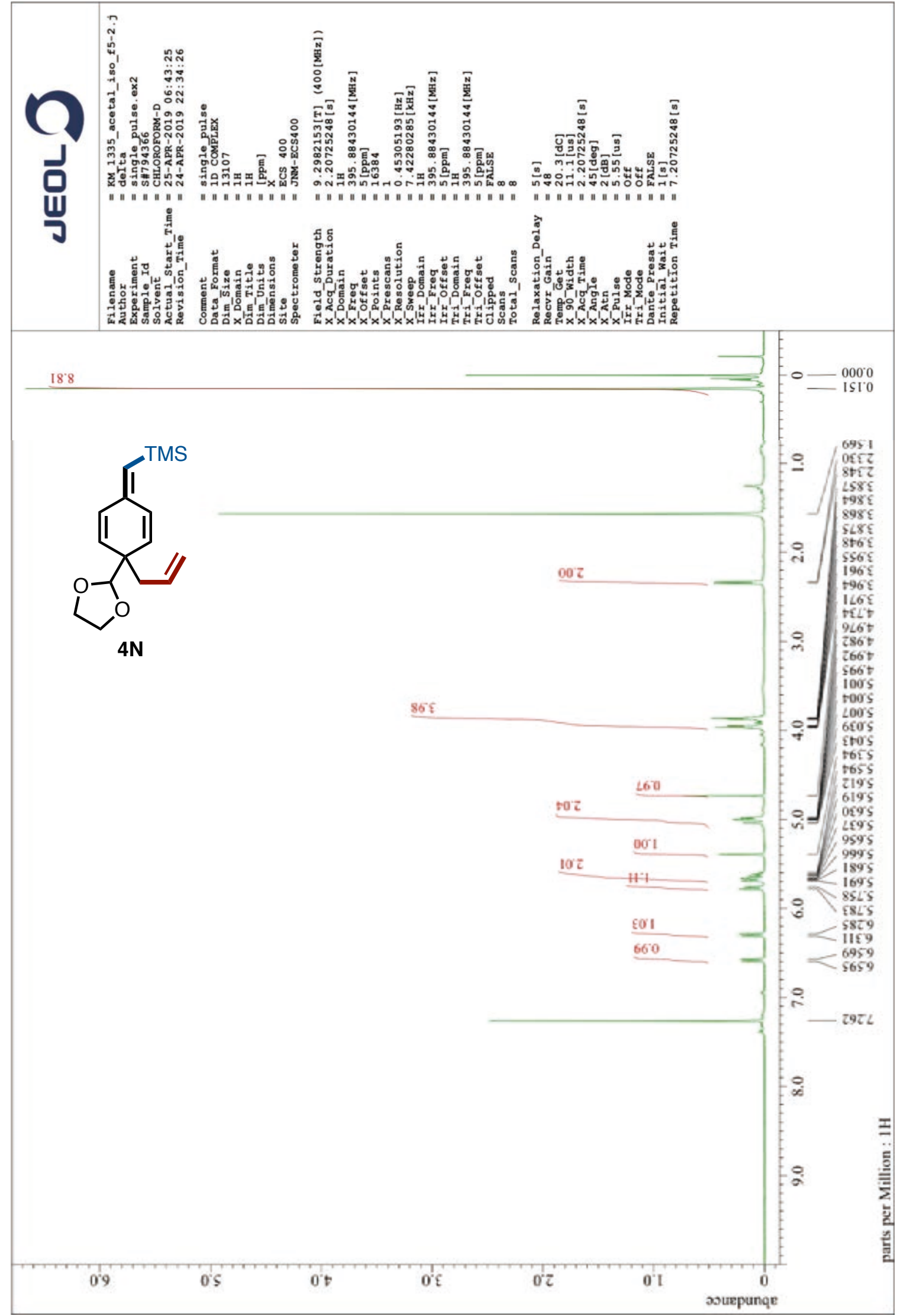


${ }^{13} \mathrm{C}$ NMR of $\mathbf{4 N}\left(101 \mathrm{MHz}, \mathrm{CDCl}_{3}\right)$

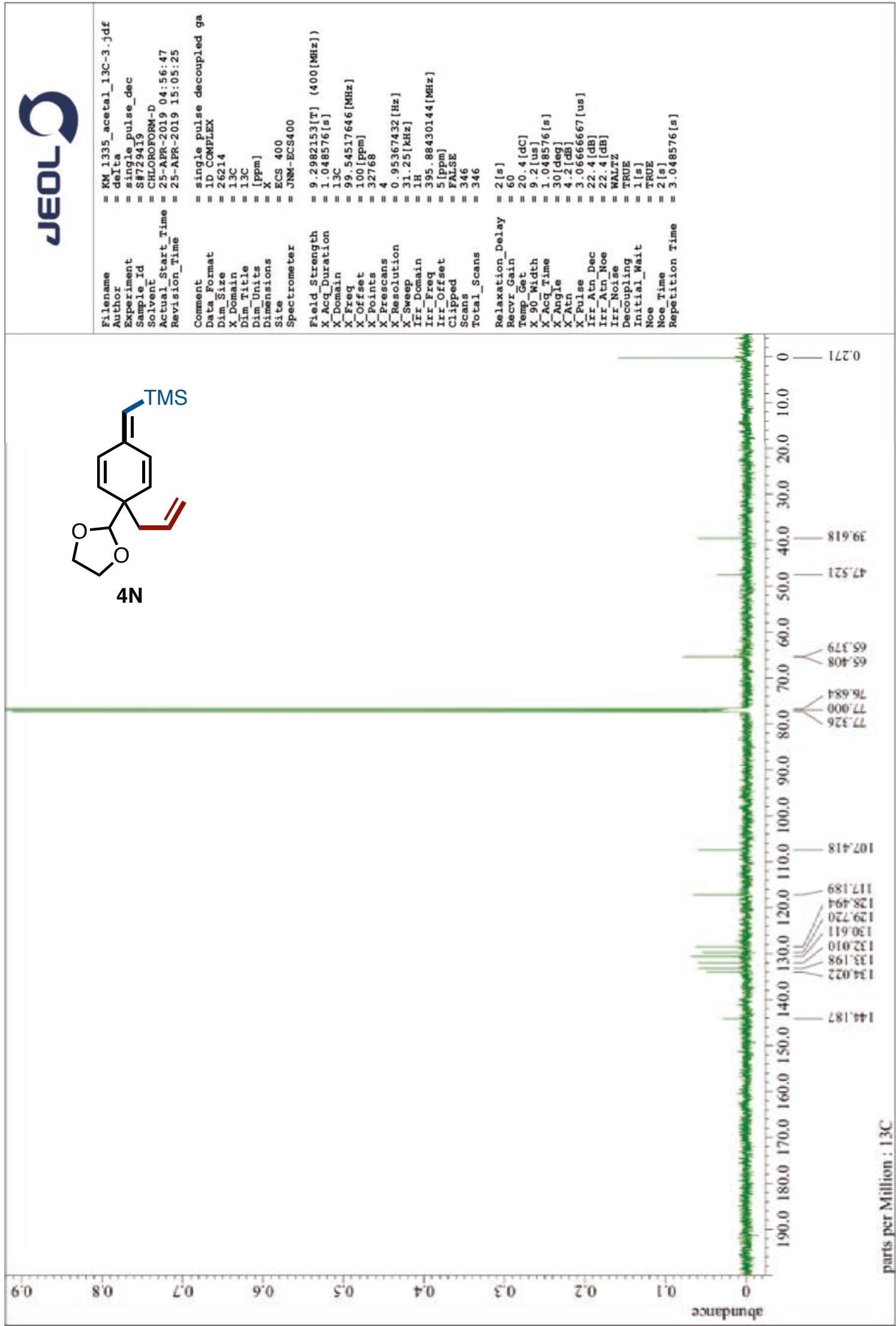


${ }^{1} \mathrm{H}$ NMR of $40\left(400 \mathrm{MHz}, \mathrm{CDCl}_{3}\right)$

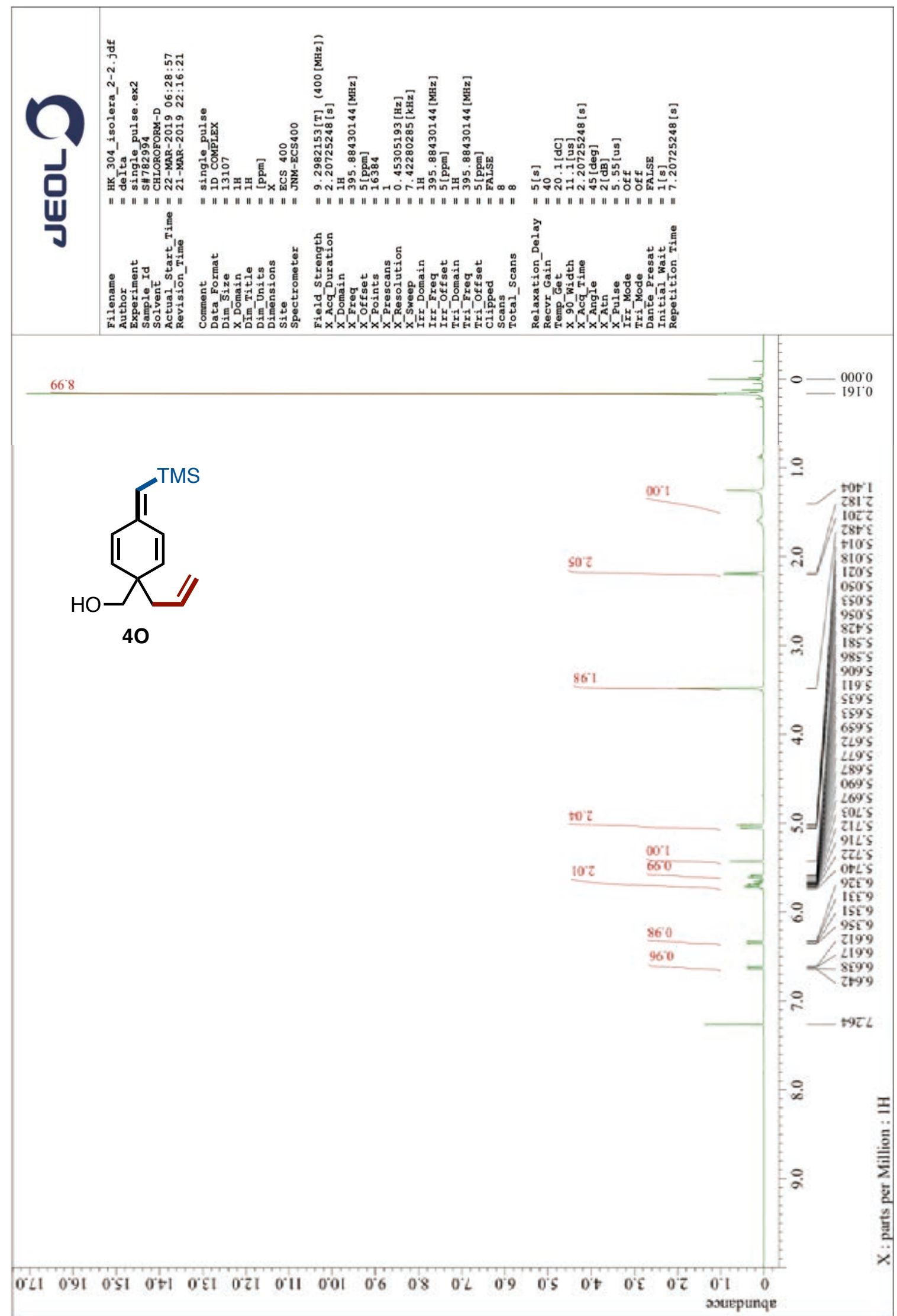


${ }^{13} \mathrm{C}$ NMR of $40\left(101 \mathrm{MHz}, \mathrm{CDCl}_{3}\right)$

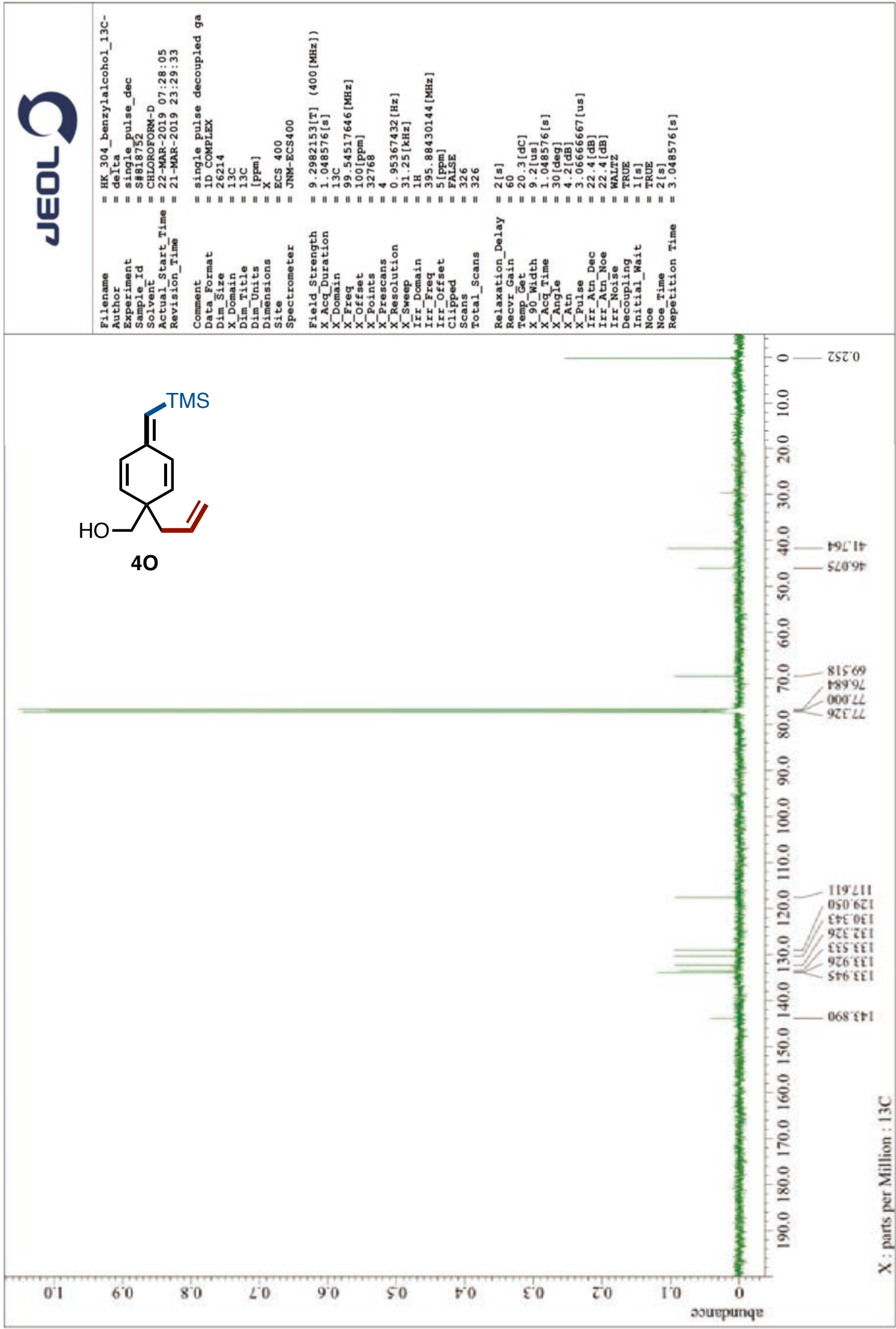


${ }^{1} \mathrm{H}$ NMR of 4P' (400 MHz, $\left.\mathrm{CDCl}_{3}\right)$

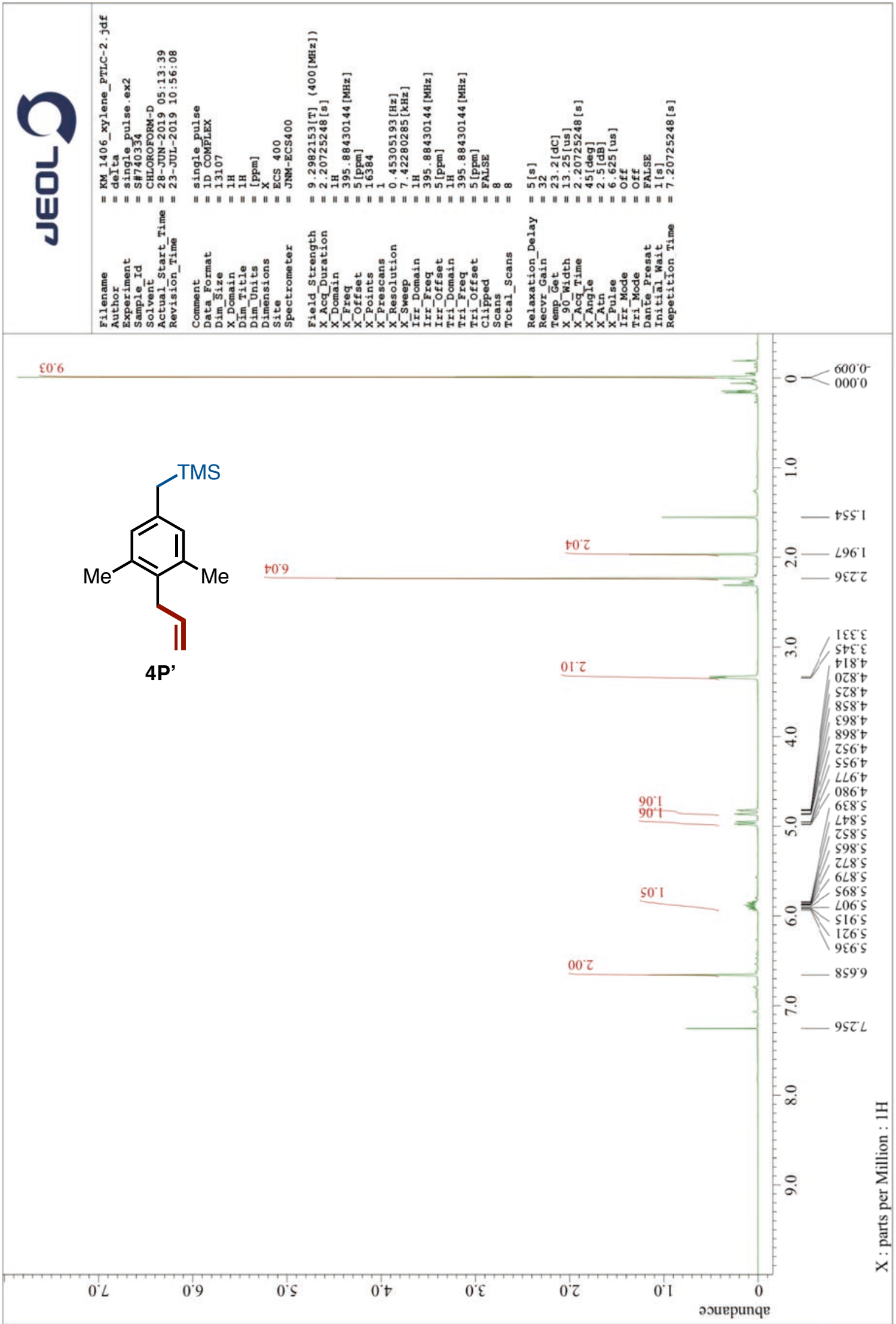


${ }^{13} \mathrm{C}$ NMR of $4 \mathbf{P} '\left(101 \mathrm{MHz}, \mathrm{CDCl}_{3}\right)$

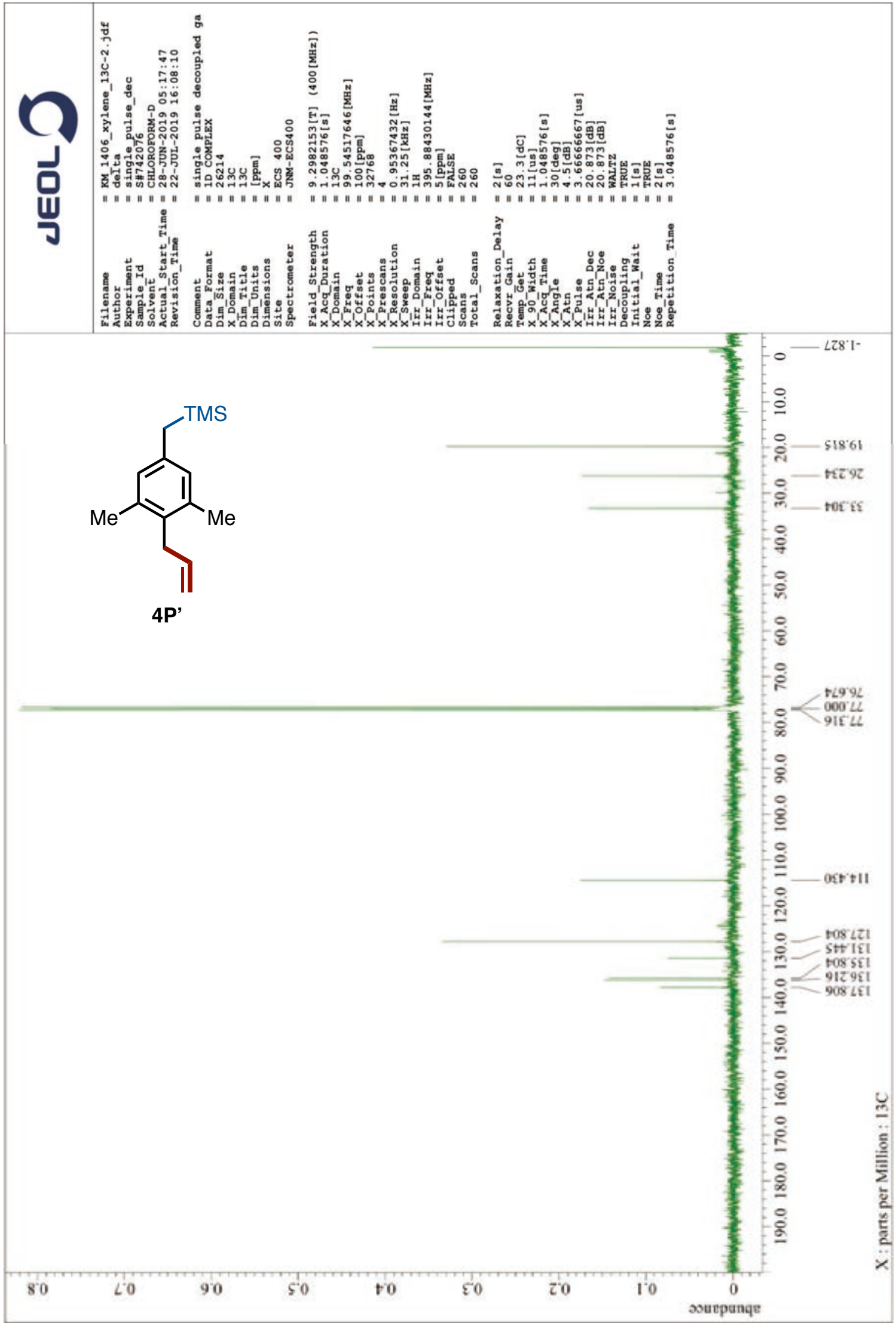


${ }^{1} \mathrm{H}$ NMR of 4Q' (400 MHz, $\mathrm{CDCl}_{3}$ )

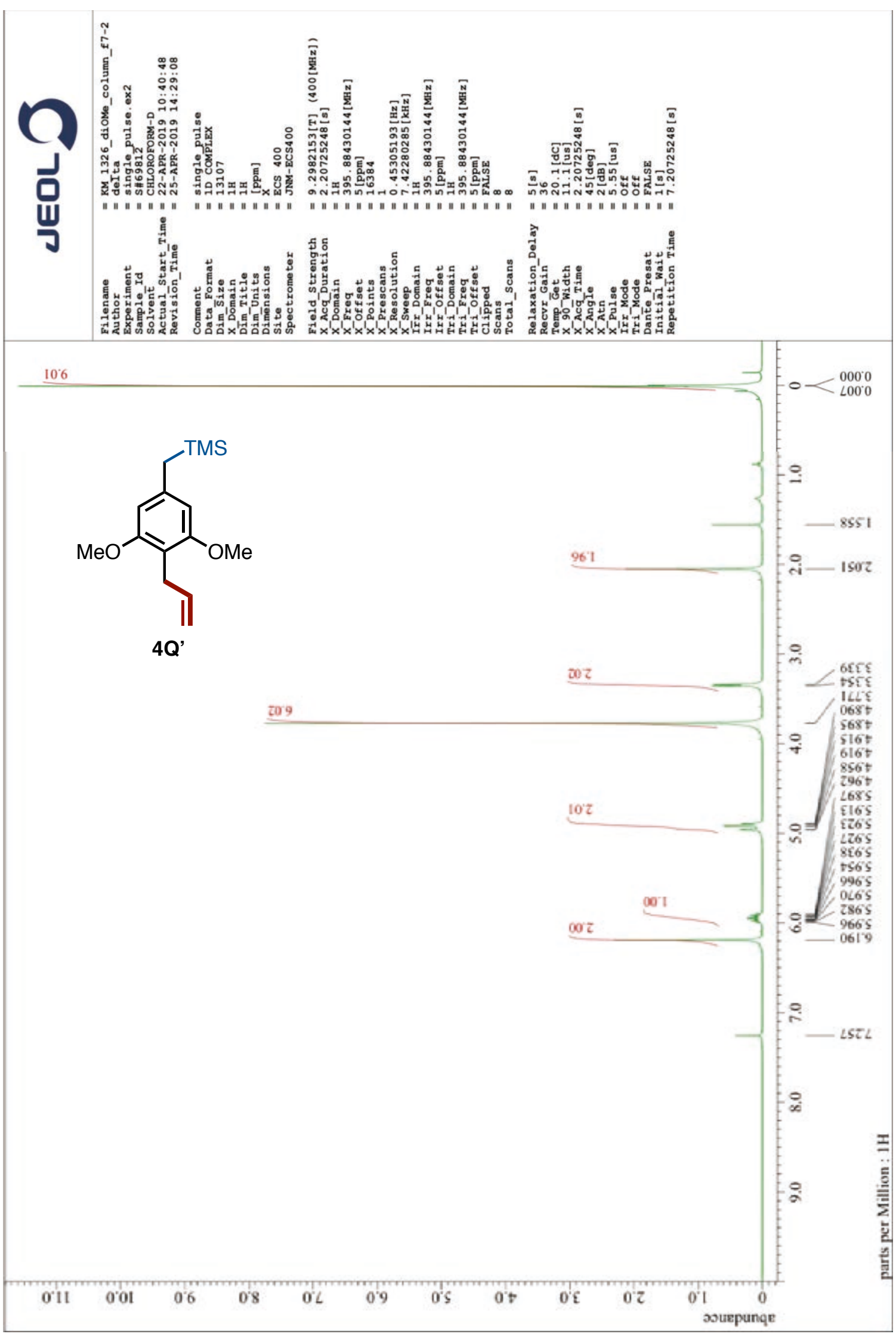


${ }^{13} \mathrm{C}$ NMR of 4Q' $\left(101 \mathrm{MHz}, \mathrm{CDCl}_{3}\right)$

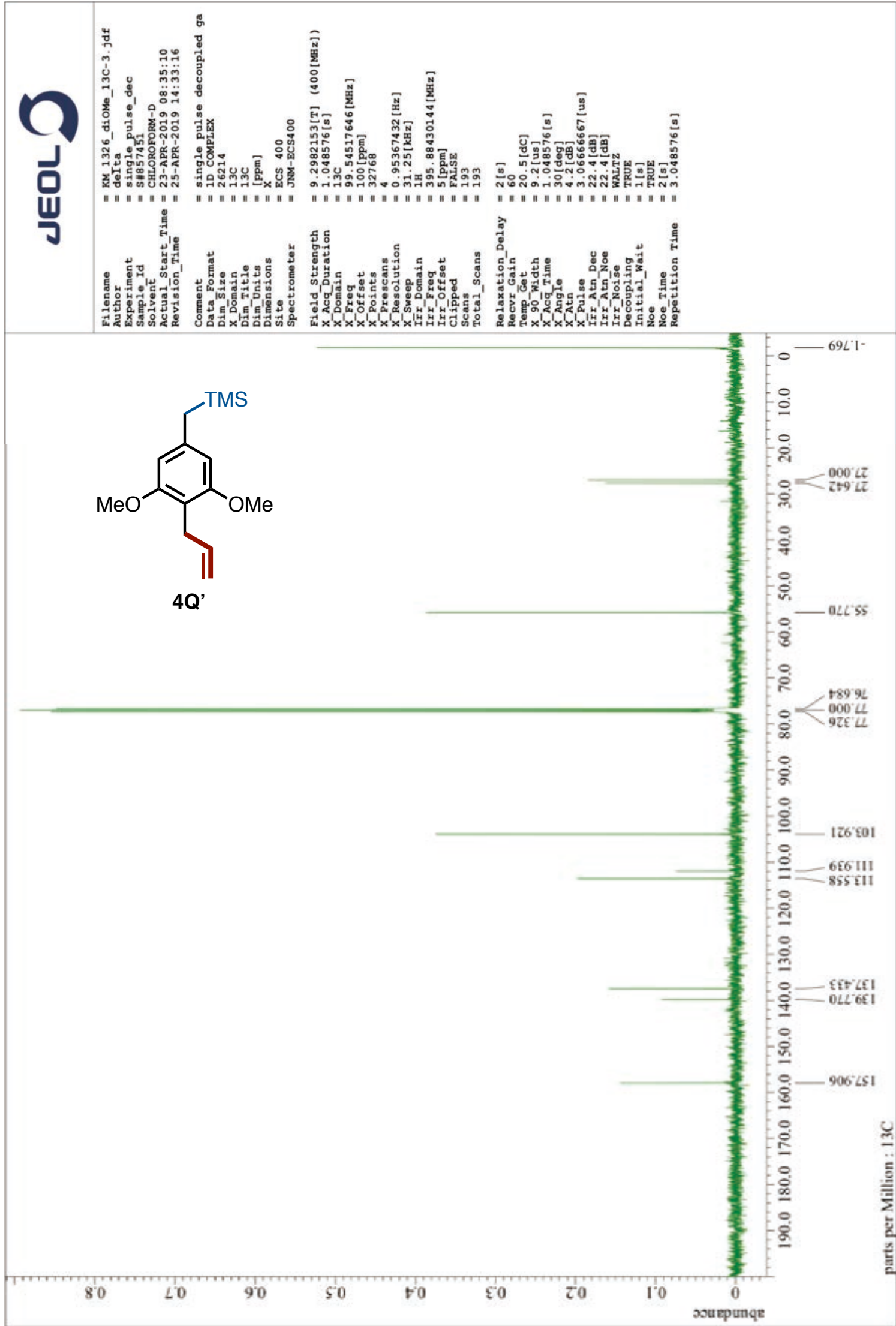


${ }^{1} \mathrm{H}$ NMR of $4 \mathbf{R}\left(400 \mathrm{MHz}, \mathrm{CDCl}_{3}\right)$

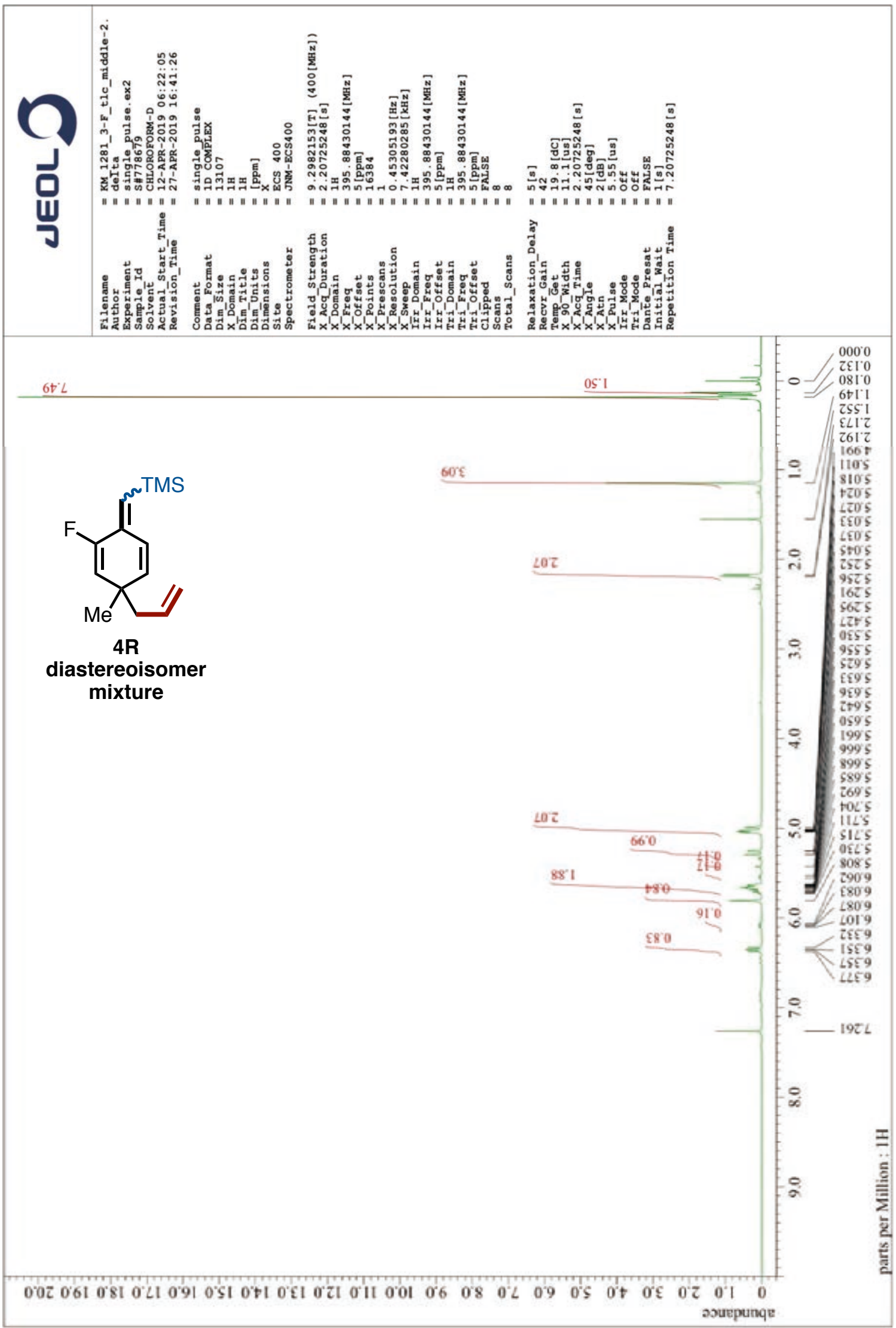


${ }^{13} \mathrm{C}$ NMR of $4 \mathbf{R}\left(101 \mathrm{MHz}, \mathrm{CDCl}_{3}\right)$

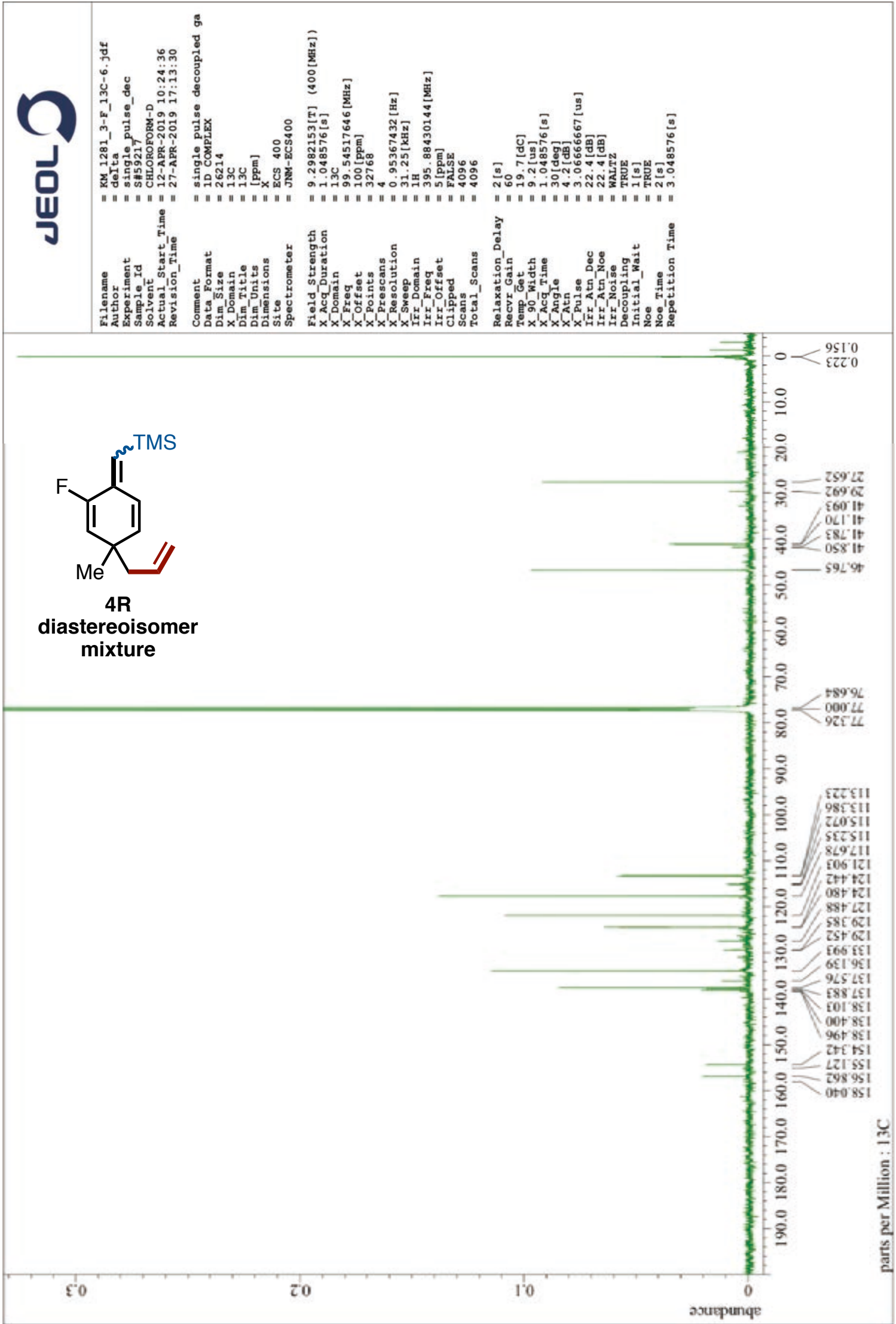


${ }^{1} \mathrm{H}$ NMR of $\mathbf{4 S}\left(400 \mathrm{MHz}, \mathrm{CDCl}_{3}\right)$

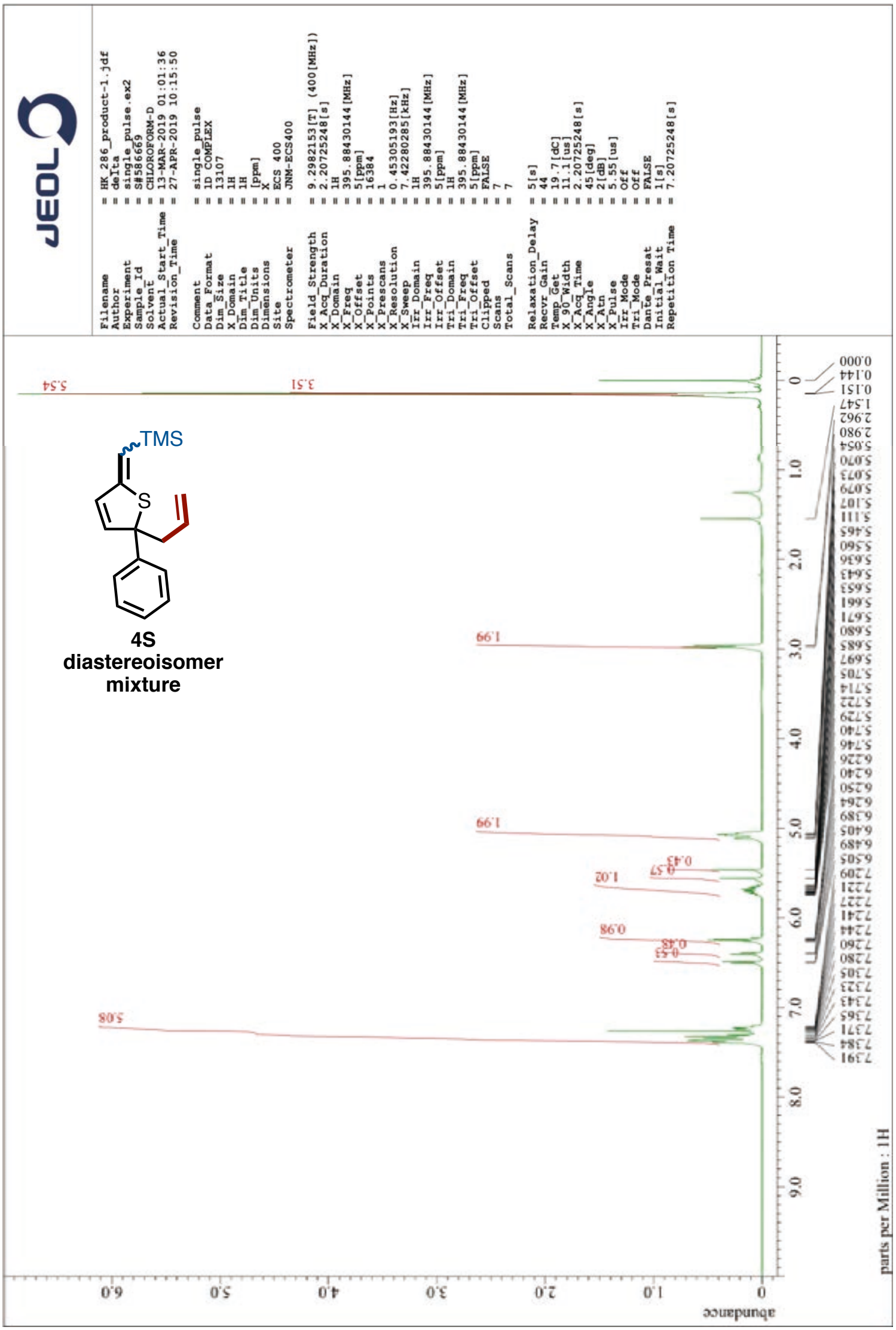


${ }^{13} \mathrm{C}$ NMR of $4 \mathrm{~S}\left(101 \mathrm{MHz}, \mathrm{CDCl}_{3}\right)$

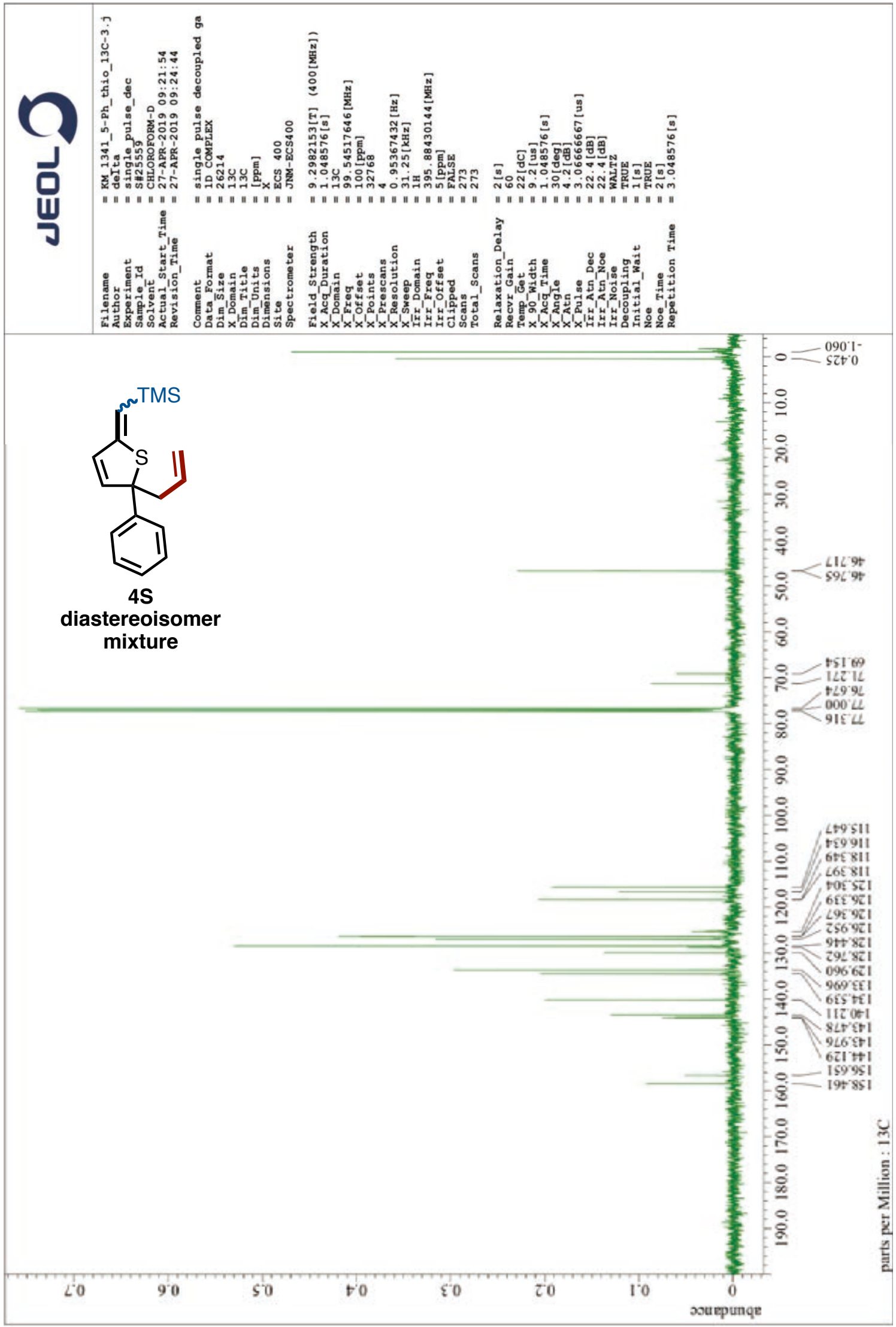


${ }^{1} \mathrm{H}$ NMR of 4 T $\left(400 \mathrm{MHz}, \mathrm{CDCl}_{3}\right)$

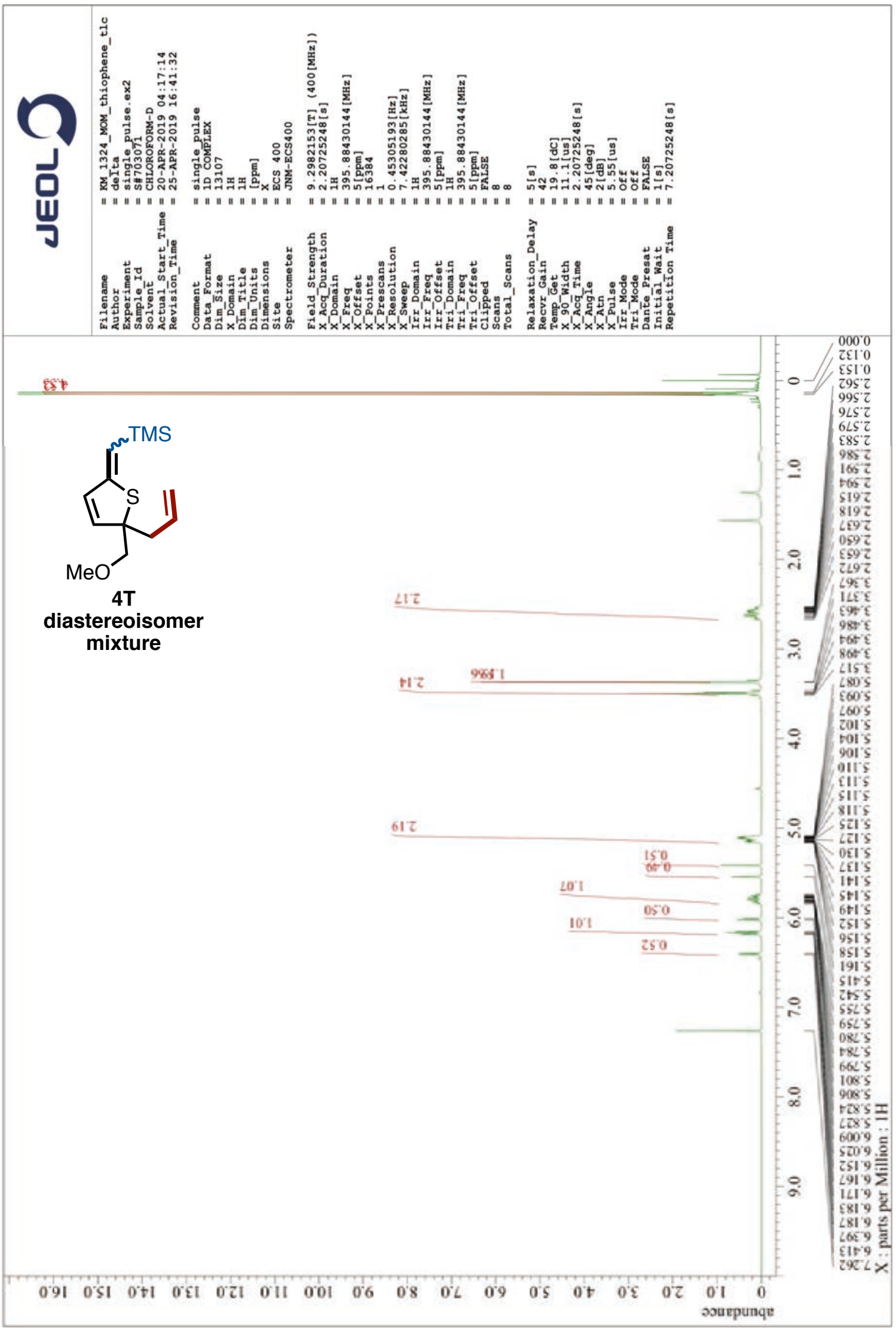


${ }^{13} \mathrm{C}$ NMR of $4 \mathbf{T}\left(101 \mathrm{MHz}, \mathrm{CDCl}_{3}\right)$

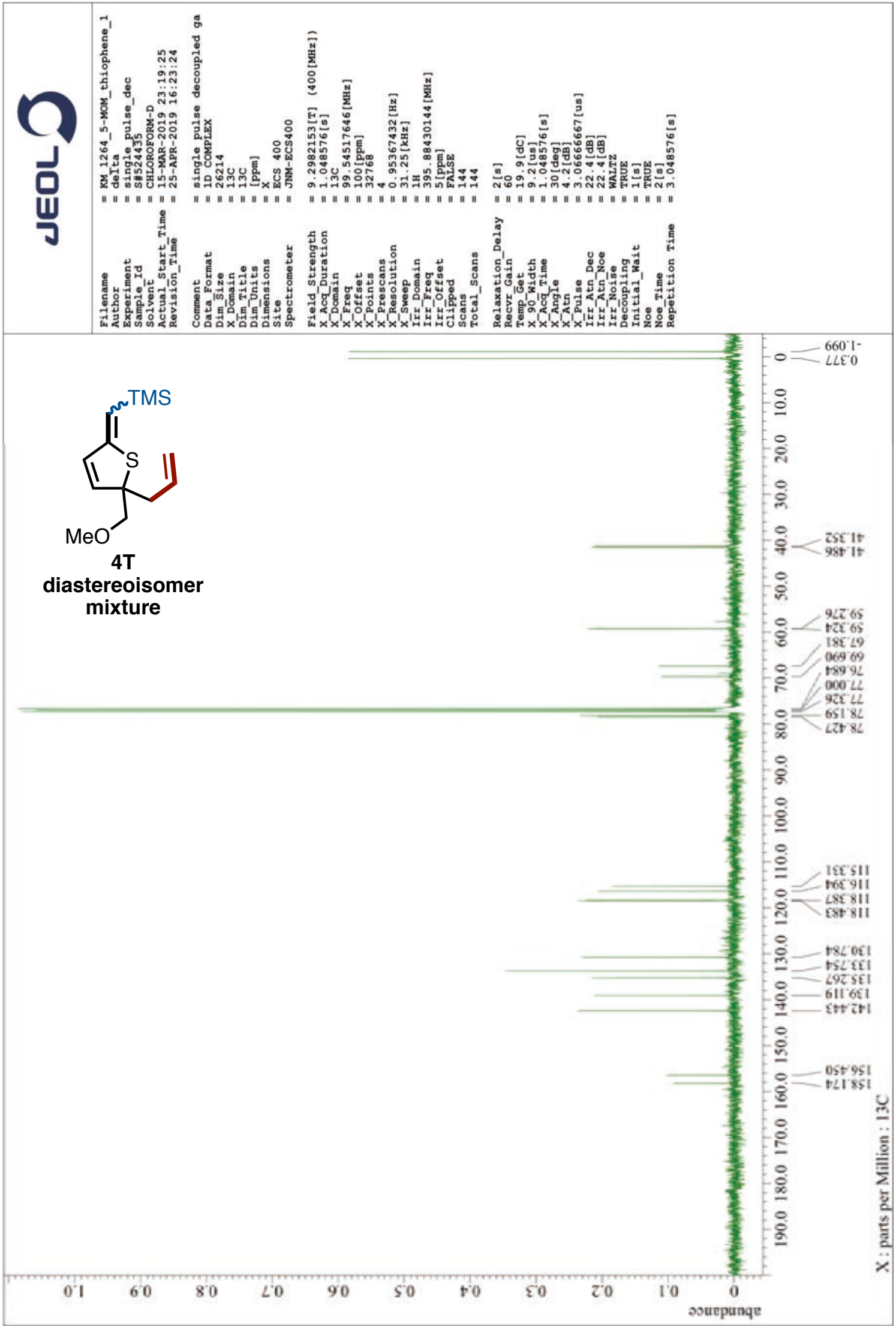


${ }^{1} \mathrm{H}$ NMR of $\mathbf{4} \mathbf{U}\left(400 \mathrm{MHz}, \mathrm{CDCl}_{3}\right)$

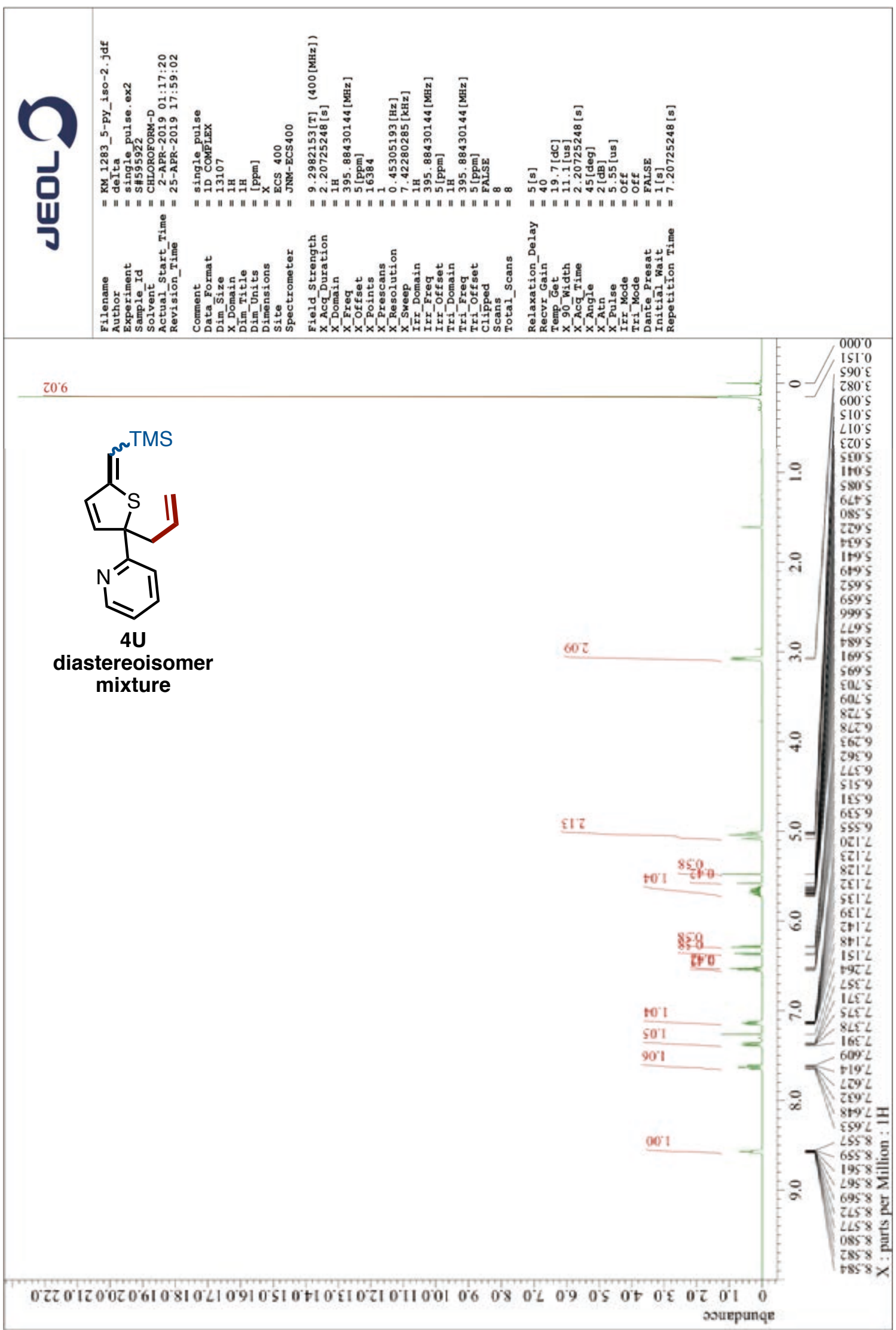


${ }^{13} \mathrm{C}$ NMR of $\mathbf{4} \mathbf{U}\left(101 \mathrm{MHz}, \mathrm{CDCl}_{3}\right)$

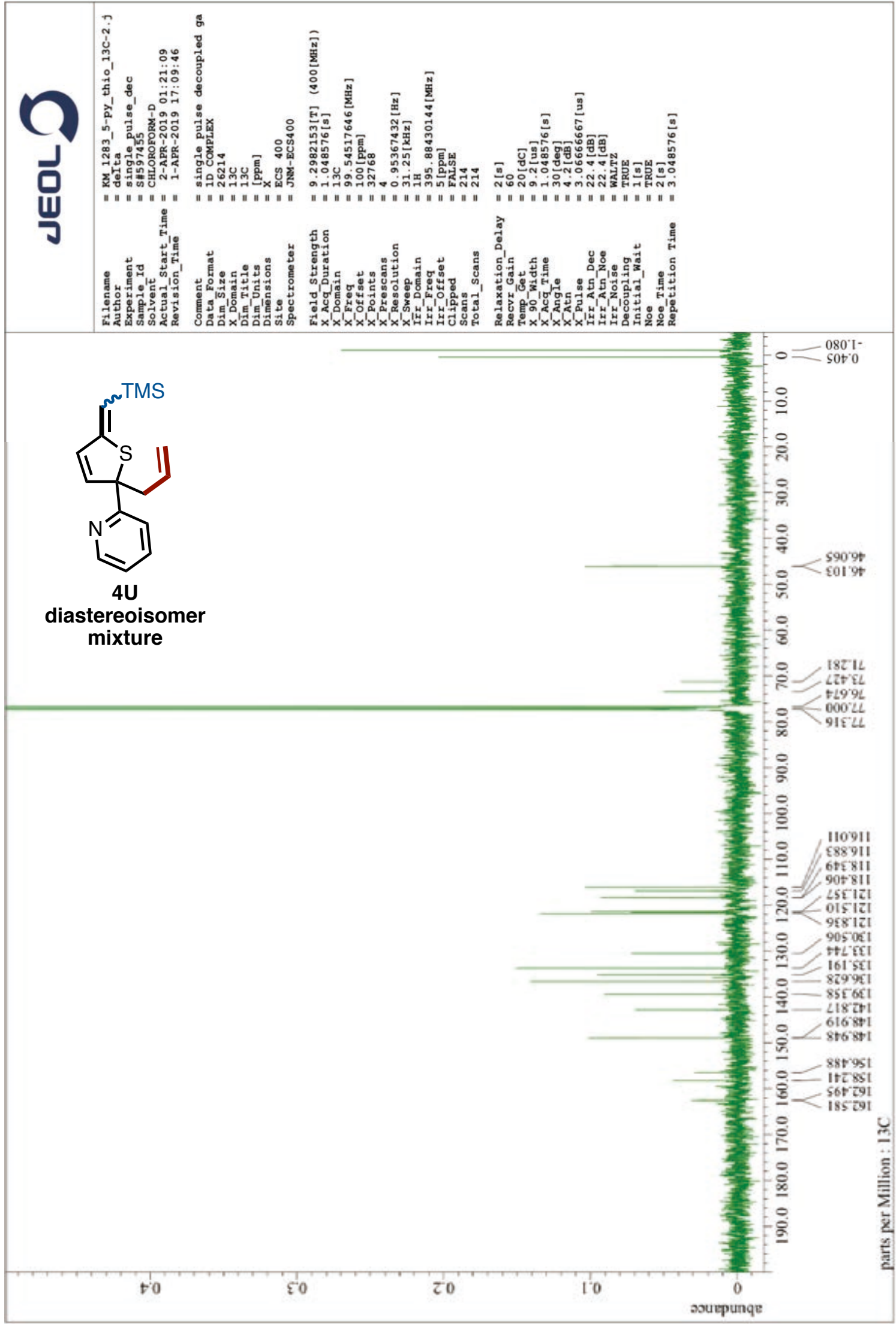


${ }^{1} \mathrm{H}$ NMR of $4 \mathbf{V}\left(400 \mathrm{MHz}, \mathrm{CDCl}_{3}\right)$

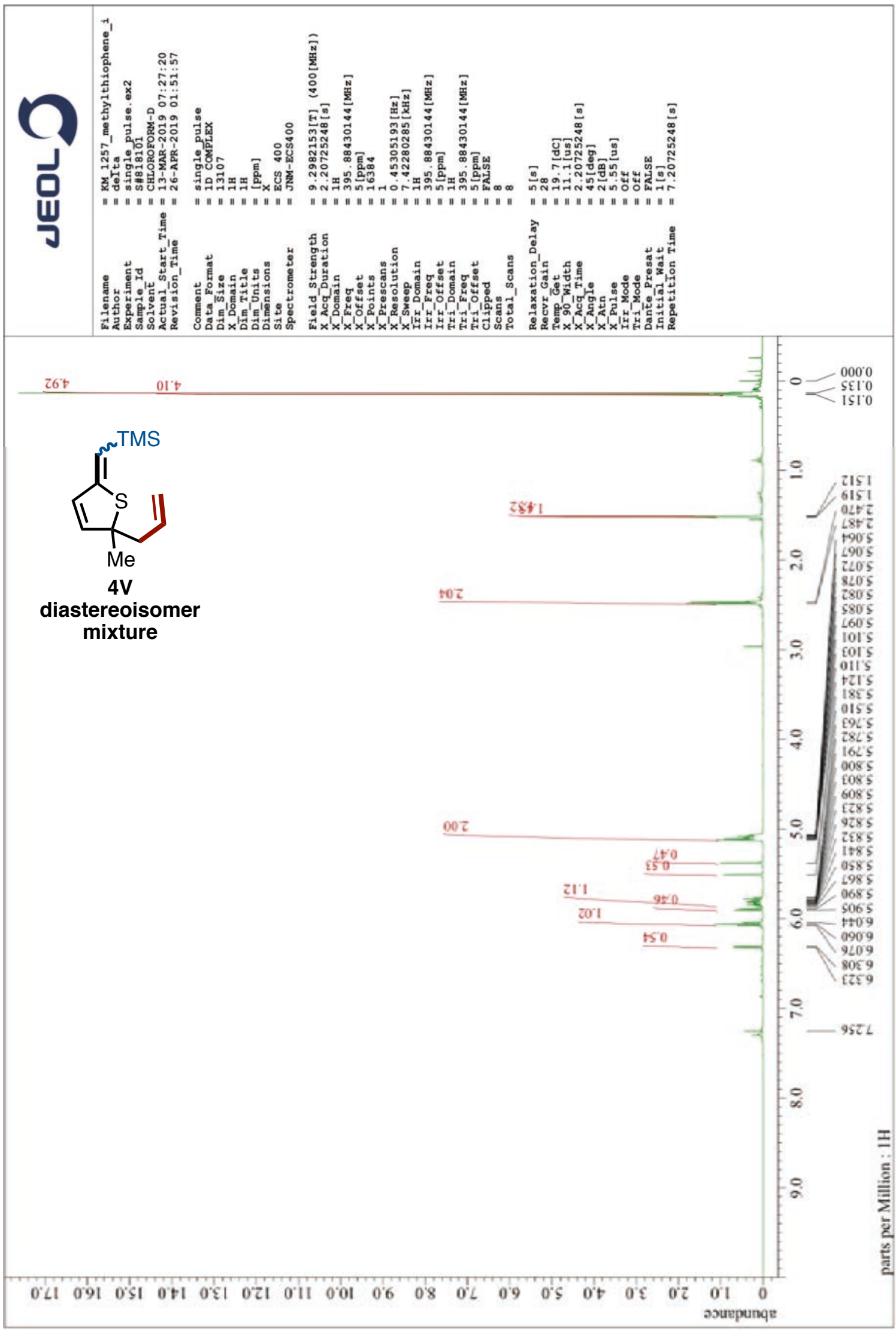


${ }^{13} \mathrm{C}$ NMR of $4 \mathbf{V}\left(101 \mathrm{MHz}, \mathrm{CDCl}_{3}\right)$

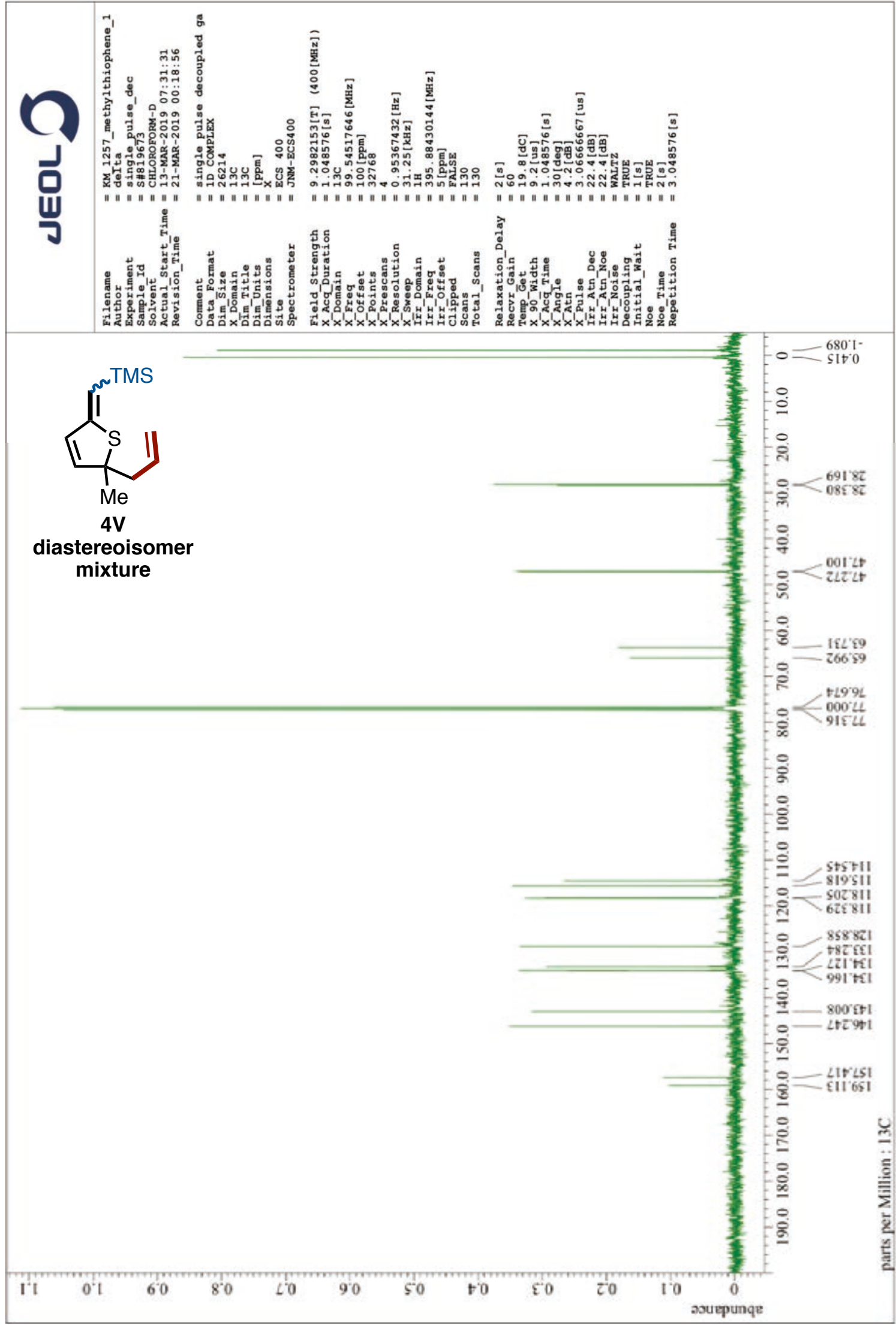


${ }^{1} \mathrm{H}$ NMR of $\mathbf{4 W}\left(400 \mathrm{MHz}, \mathrm{CDCl}_{3}\right)$

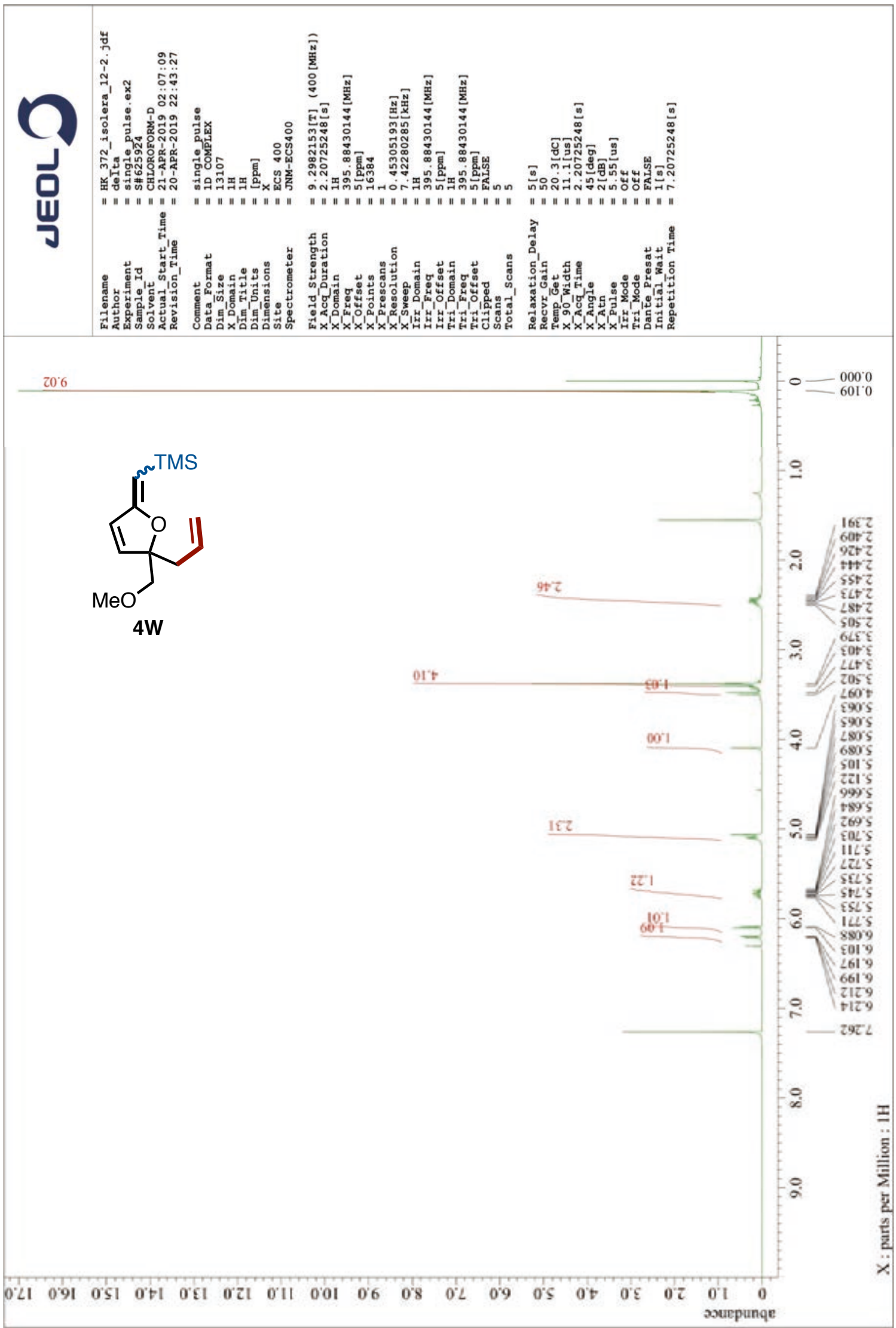


${ }^{13} \mathrm{C}$ NMR of $\mathbf{4 W}\left(101 \mathrm{MHz}, \mathrm{CDCl}_{3}\right)$

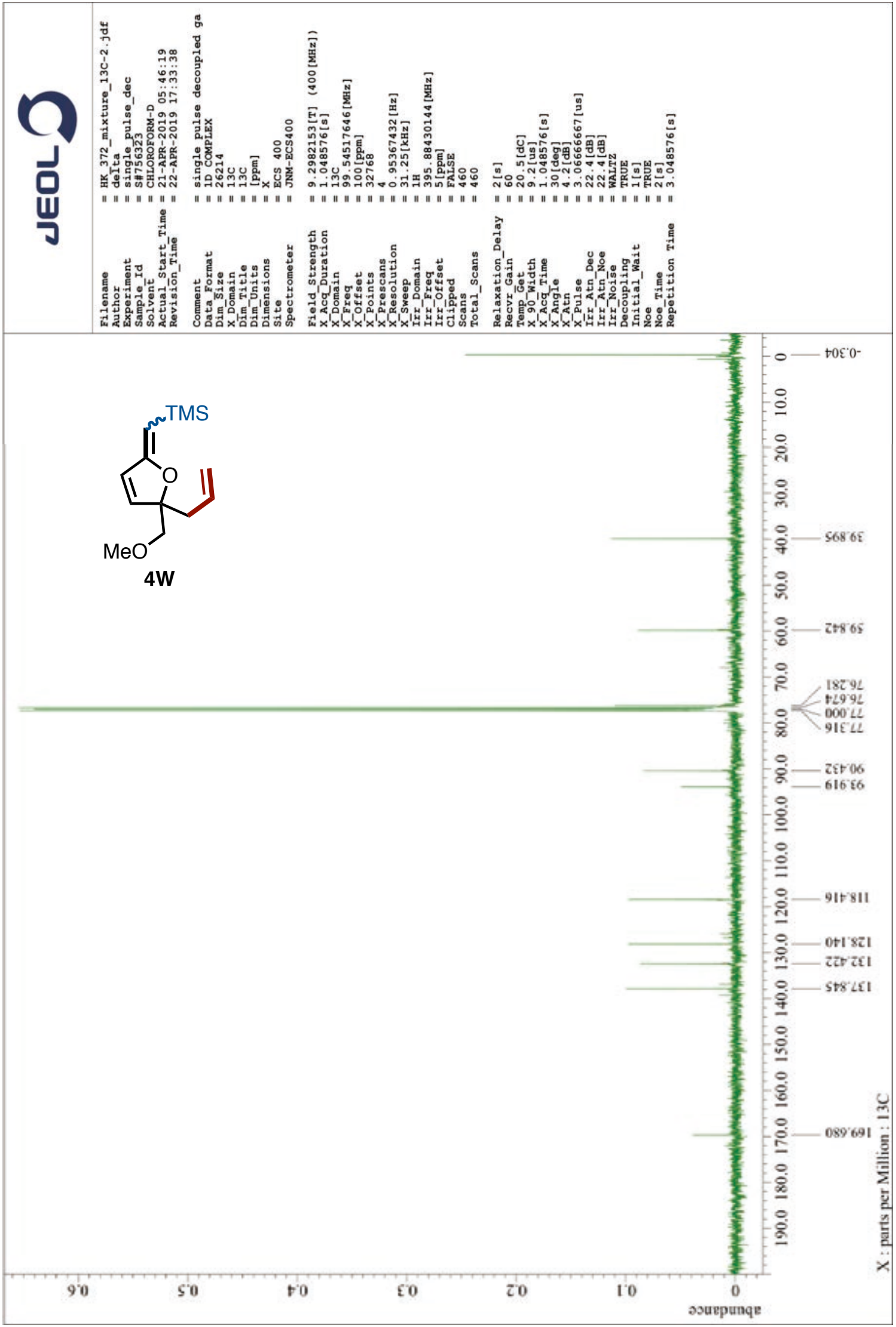


${ }^{1} \mathrm{H}$ NMR of $4 \mathbf{X}\left(400 \mathrm{MHz}, \mathrm{CDCl}_{3}\right)$

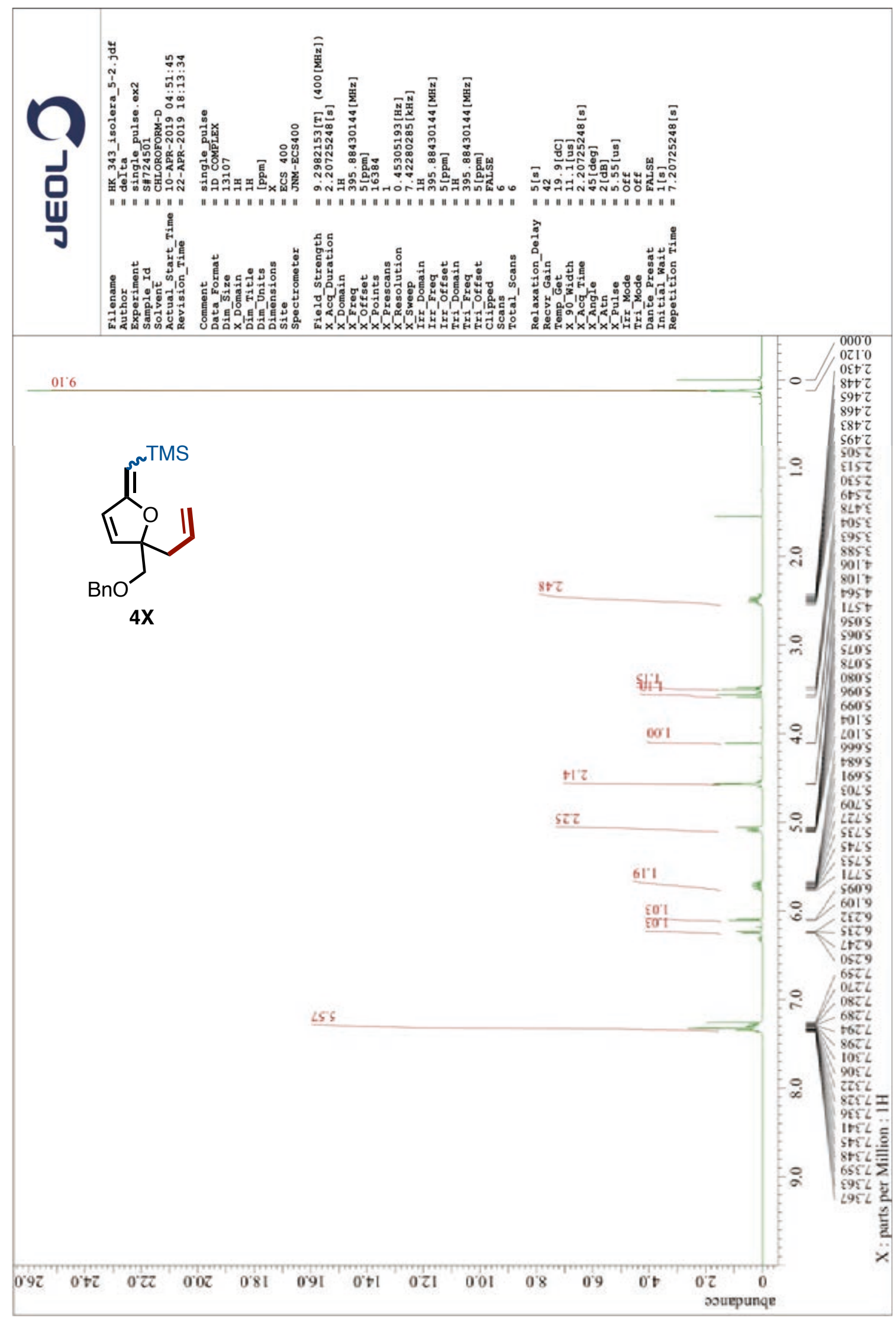


${ }^{13} \mathrm{C}$ NMR of $4 \mathbf{X}\left(101 \mathrm{MHz}, \mathrm{CDCl}_{3}\right)$

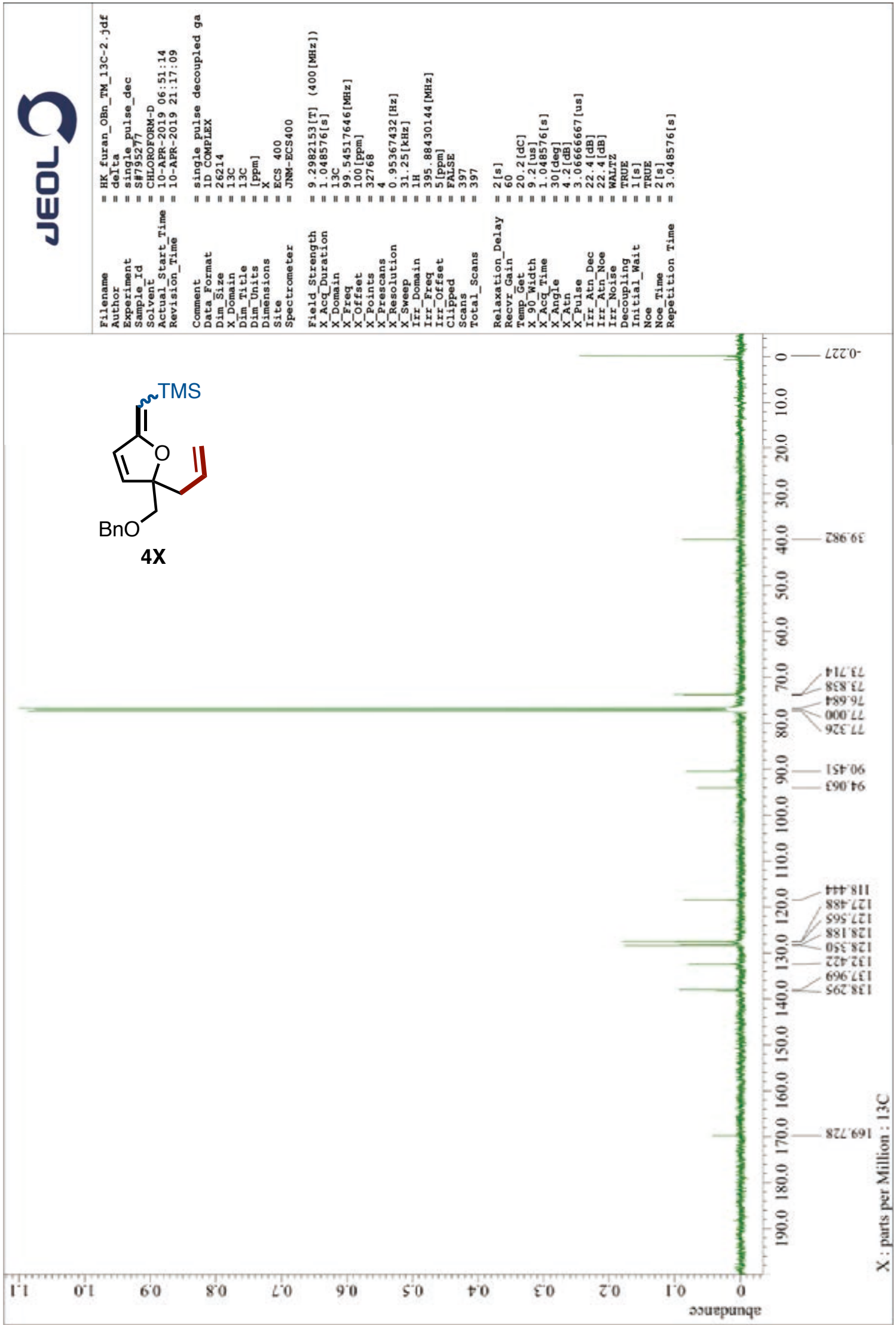


${ }^{1} \mathrm{H}$ NMR of $7\left(400 \mathrm{MHz}, \mathrm{CDCl}_{3}\right)$

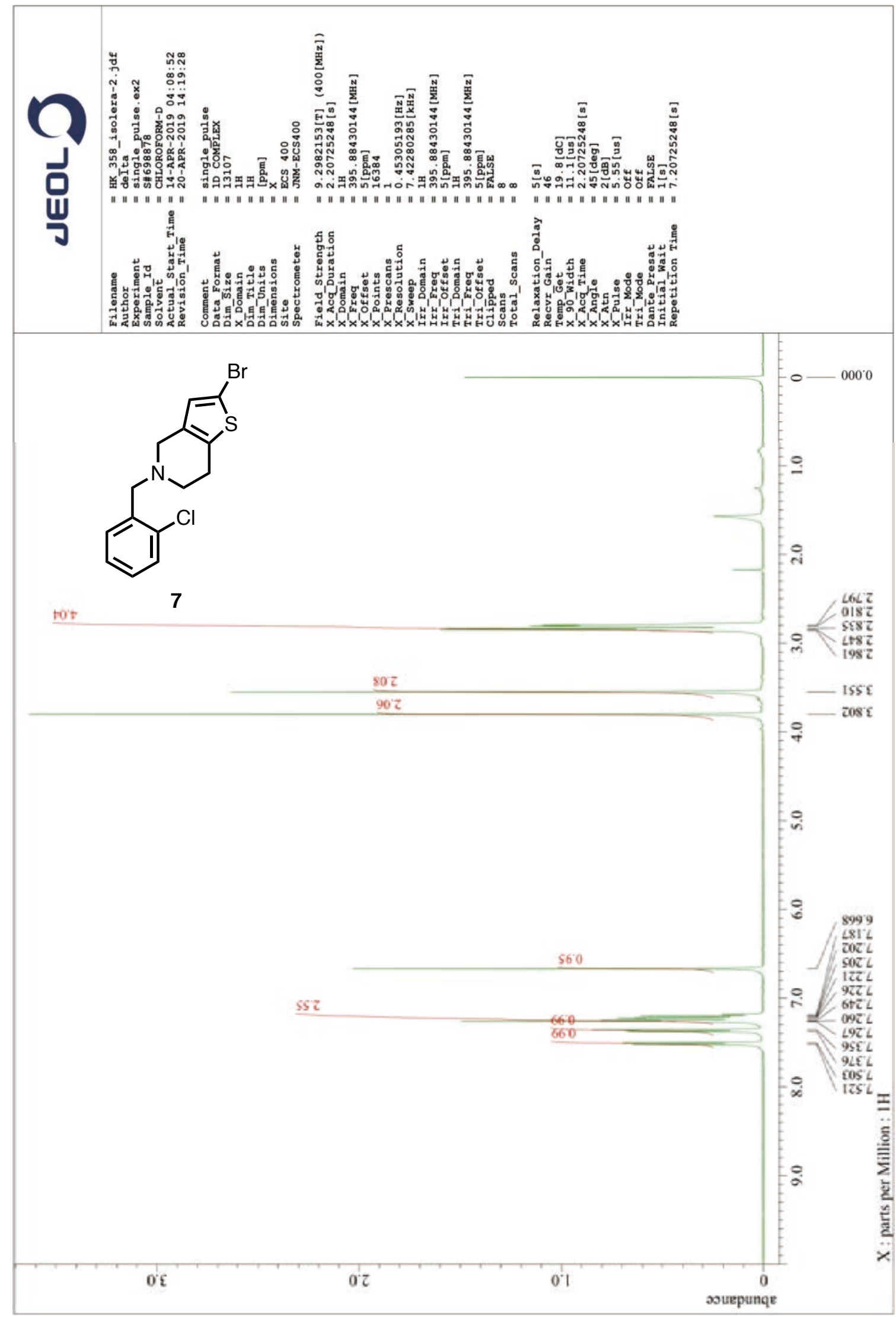


${ }^{13} \mathrm{C}$ NMR of $7\left(101 \mathrm{MHz}, \mathrm{CDCl}_{3}\right)$

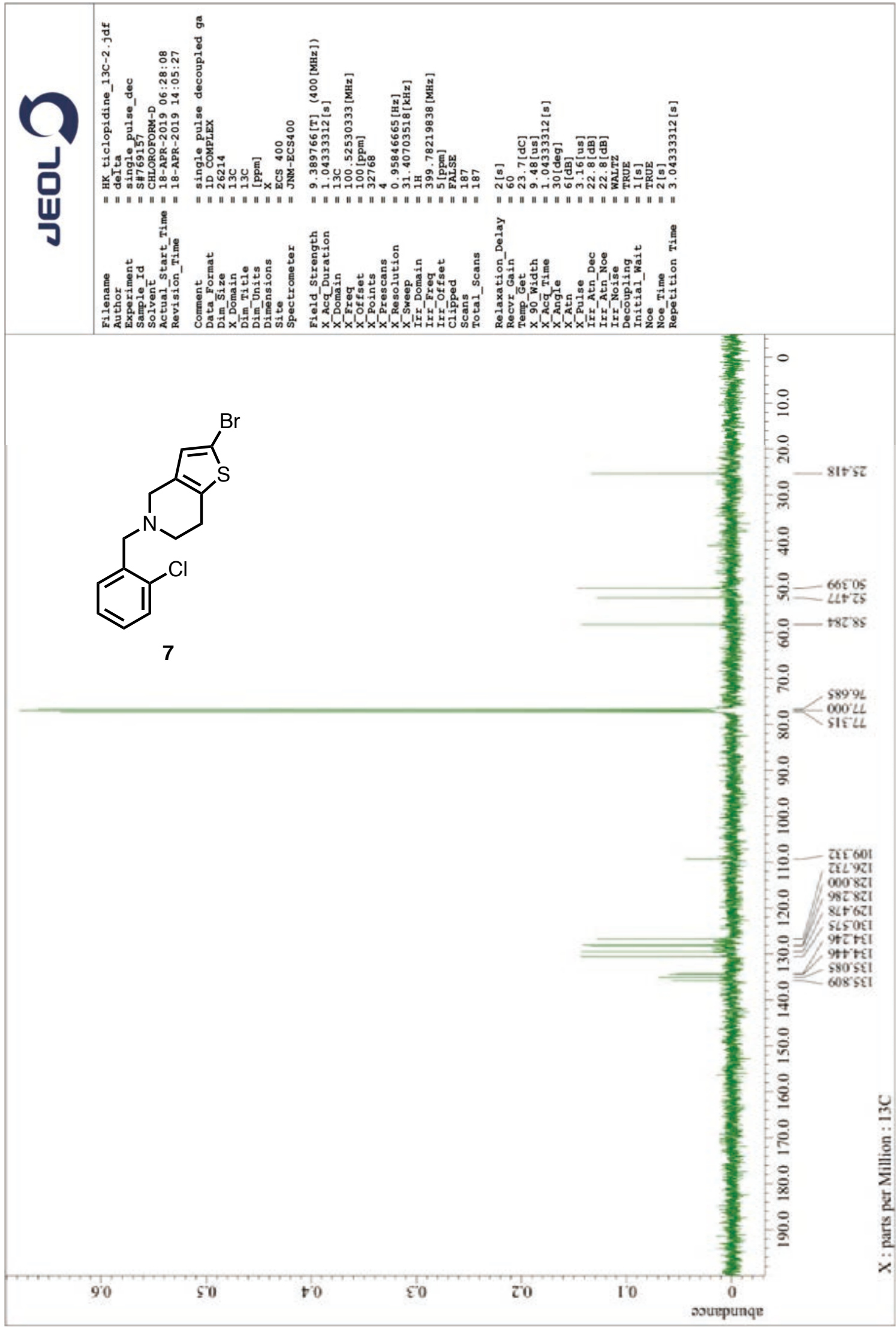


${ }^{1} \mathrm{H}$ NMR of $8\left(400 \mathrm{MHz}, \mathrm{CDCl}_{3}\right)$

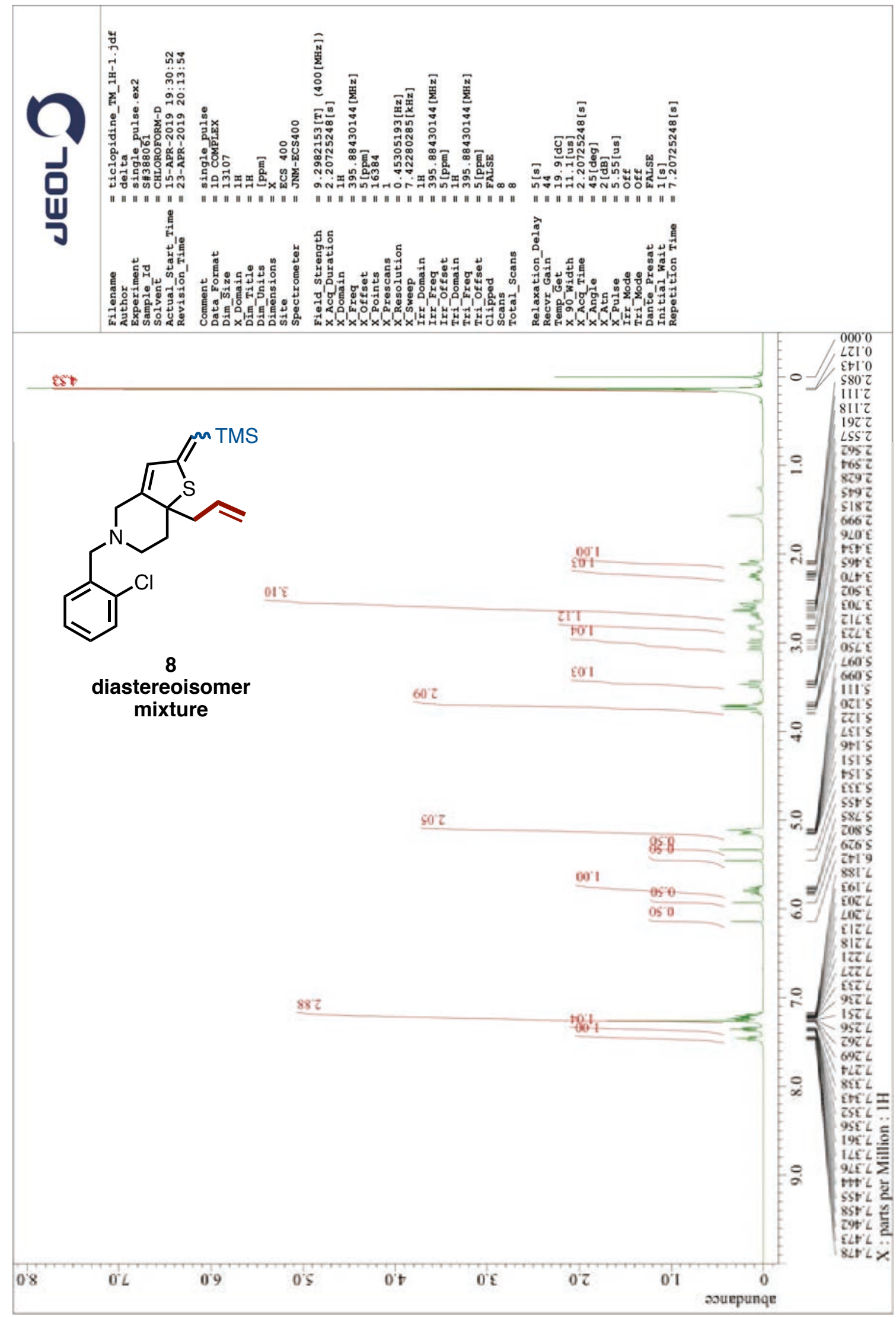


${ }^{13} \mathrm{C}$ NMR of $8\left(101 \mathrm{MHz}, \mathrm{CDCl}_{3}\right)$

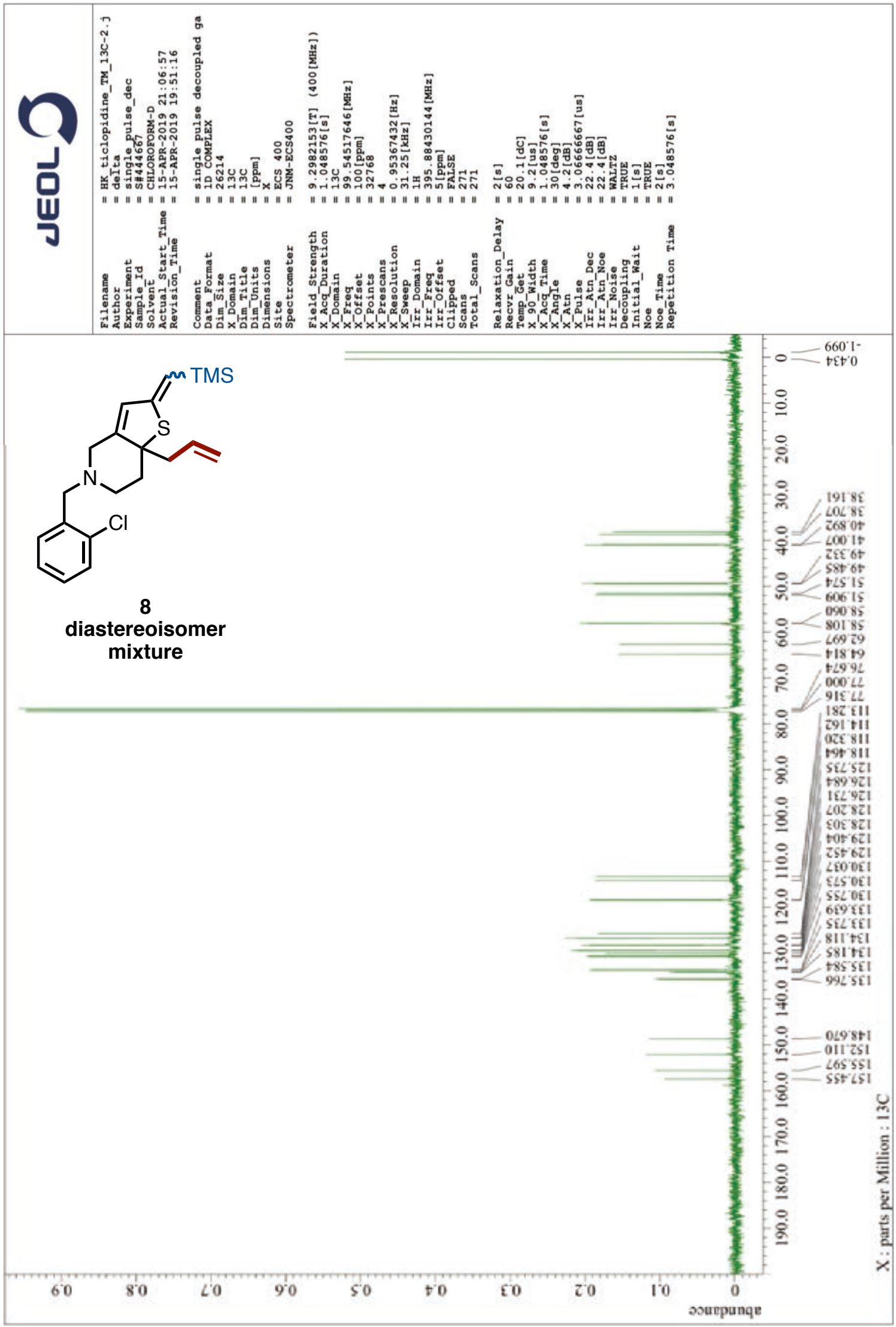


${ }^{1} \mathrm{H}$ NMR of $9\left(400 \mathrm{MHz}, \mathrm{CDCl}_{3}\right)$

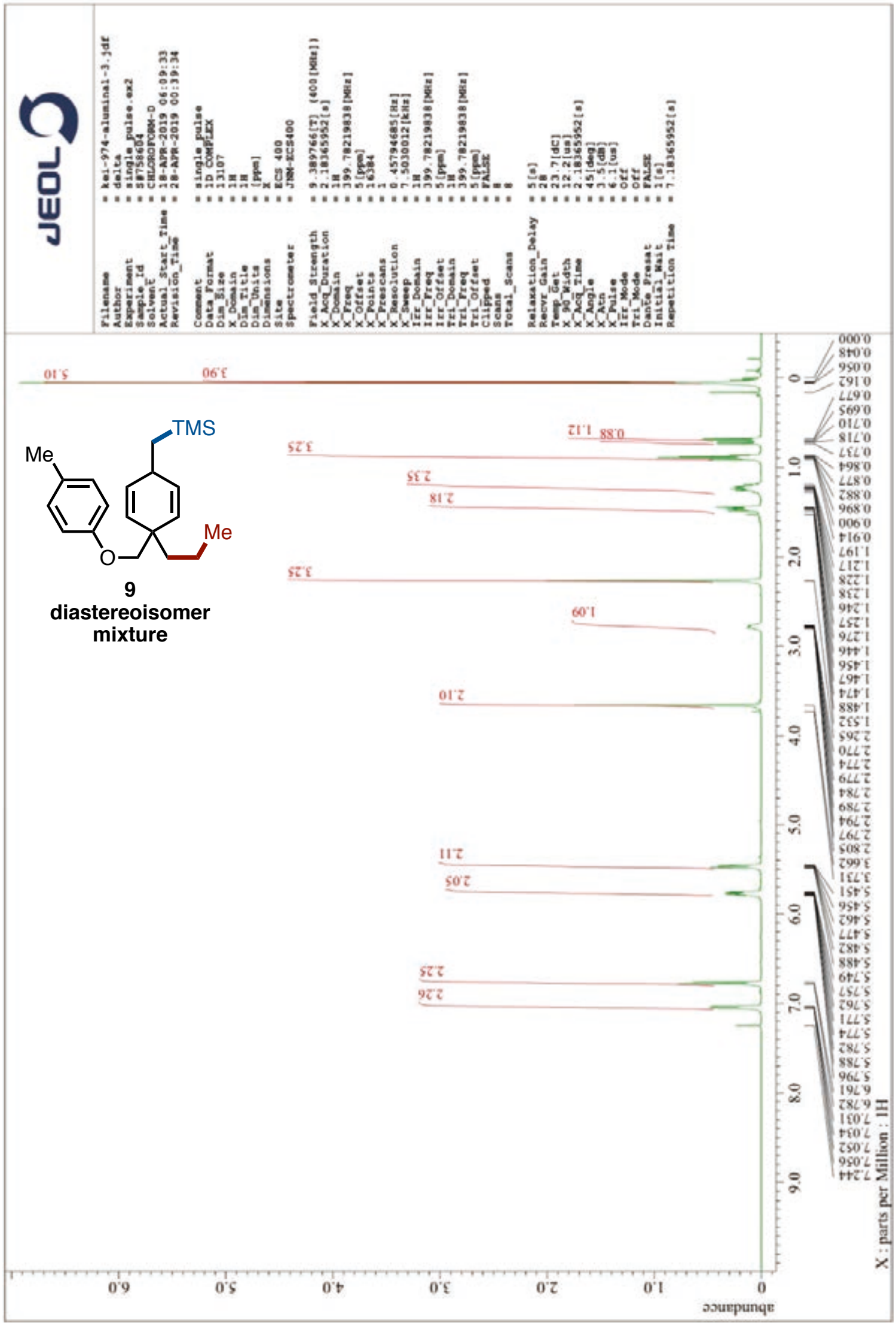


${ }^{13} \mathrm{C}$ NMR of $9\left(101 \mathrm{MHz}, \mathrm{CDCl}_{3}\right)$

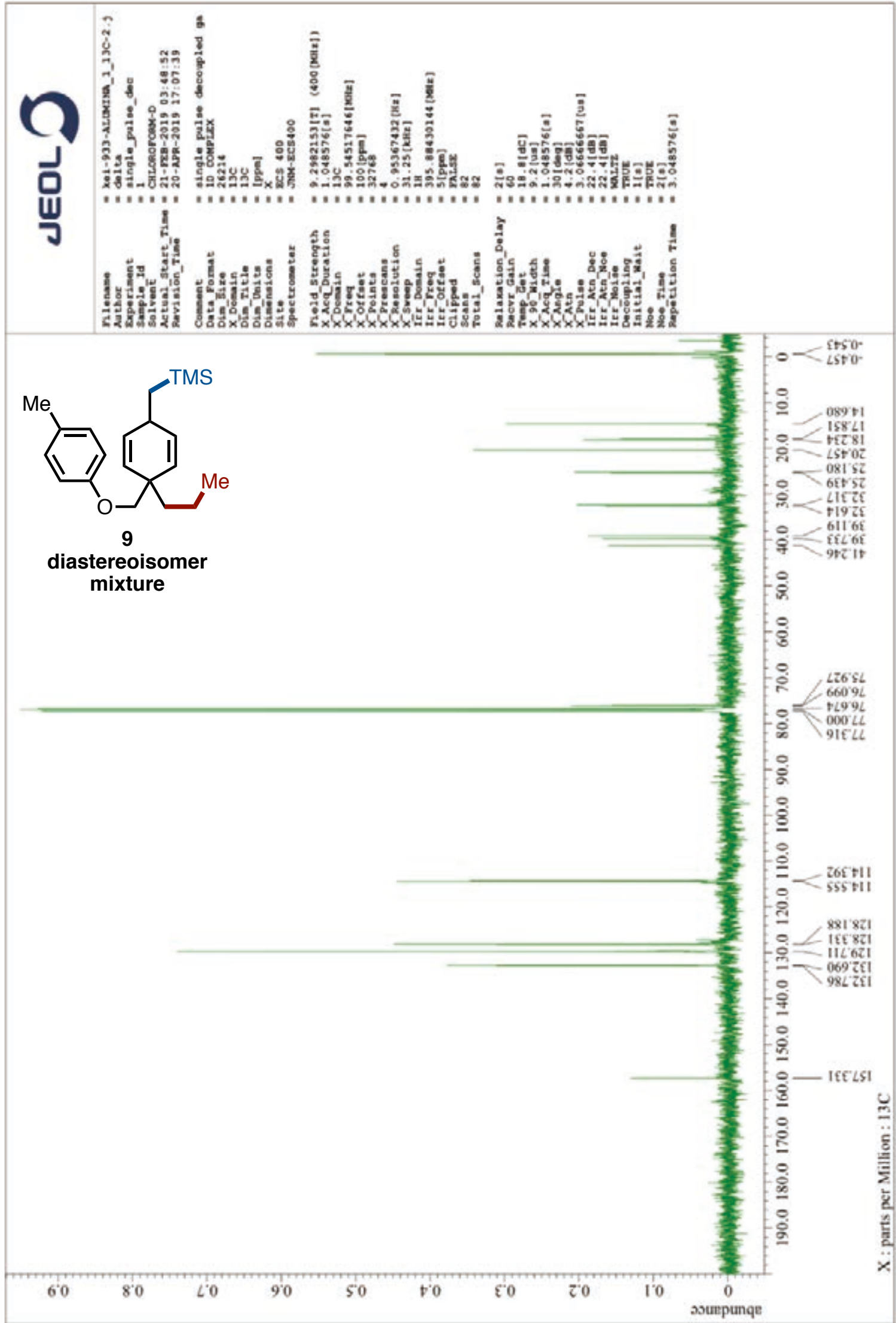


${ }^{1} \mathrm{H}$ NMR of $10\left(400 \mathrm{MHz}, \mathrm{CDCl}_{3}\right)$

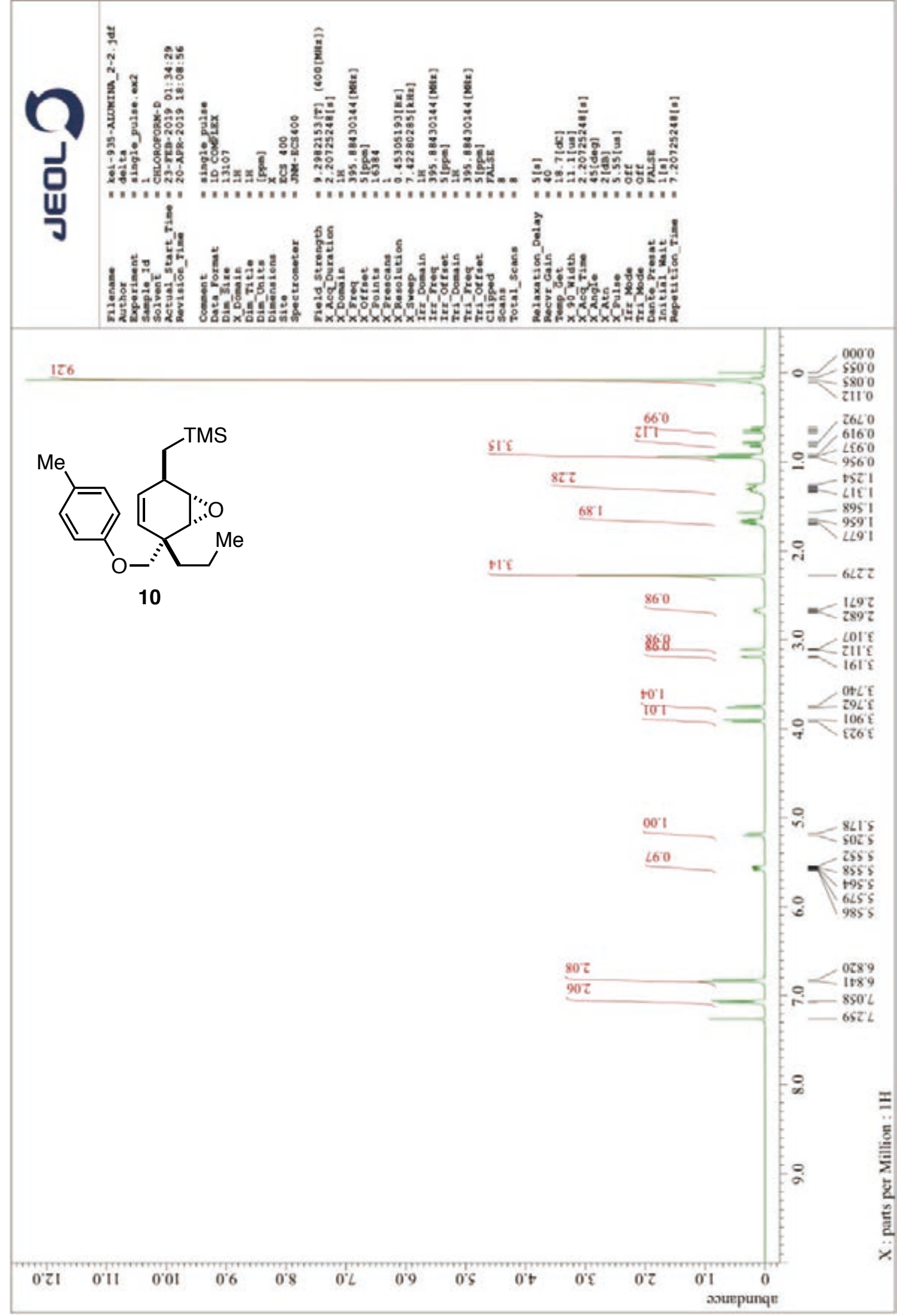


${ }^{13} \mathrm{C}$ NMR of $10\left(101 \mathrm{MHz}, \mathrm{CDCl}_{3}\right)$

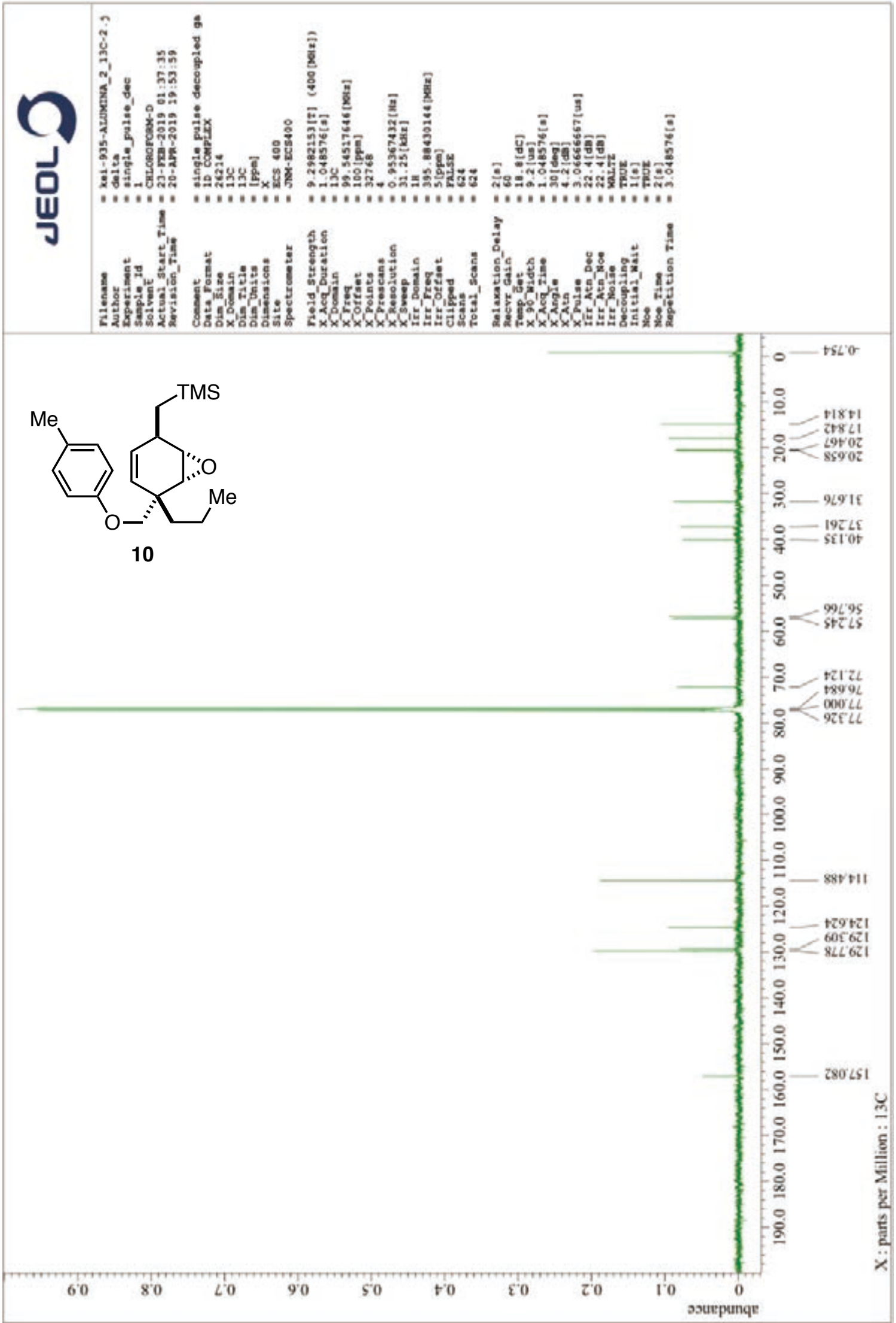


${ }^{1} \mathrm{H}$ NMR of $\mathbf{1 0 - d r}\left(400 \mathrm{MHz}, \mathrm{CDCl}_{3}\right)$

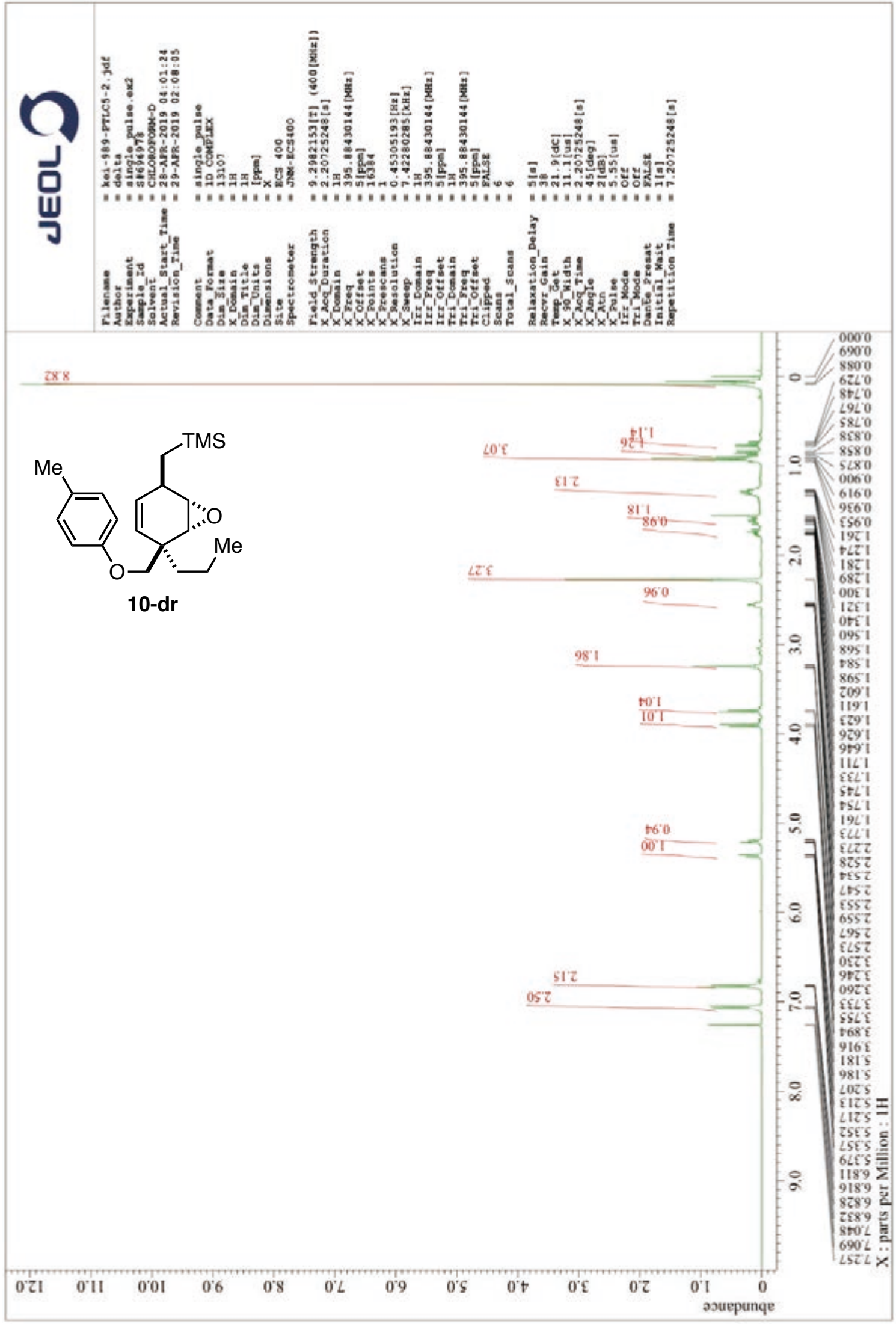


${ }^{13} \mathrm{C}$ NMR of 10-dr (101 MHz, $\left.\mathrm{CDCl}_{3}\right)$

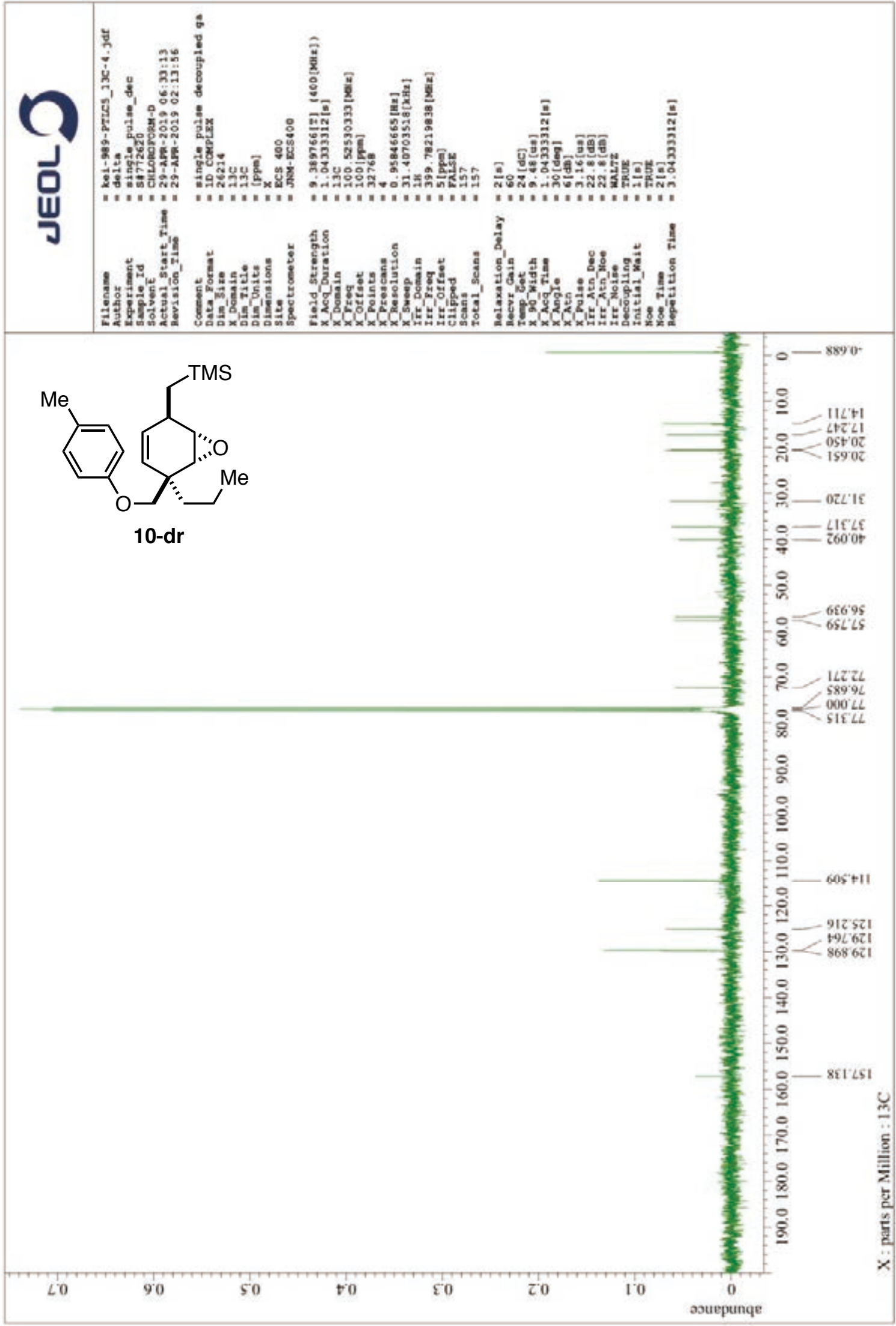


10. Crude ${ }^{1} \mathrm{H}$ NMR Spectra of 4 and 8

${ }^{1} \mathrm{H} \mathrm{NMR}$ of $\mathbf{4 A}\left(400 \mathrm{MHz}, \mathrm{CDCl}_{3}\right)$

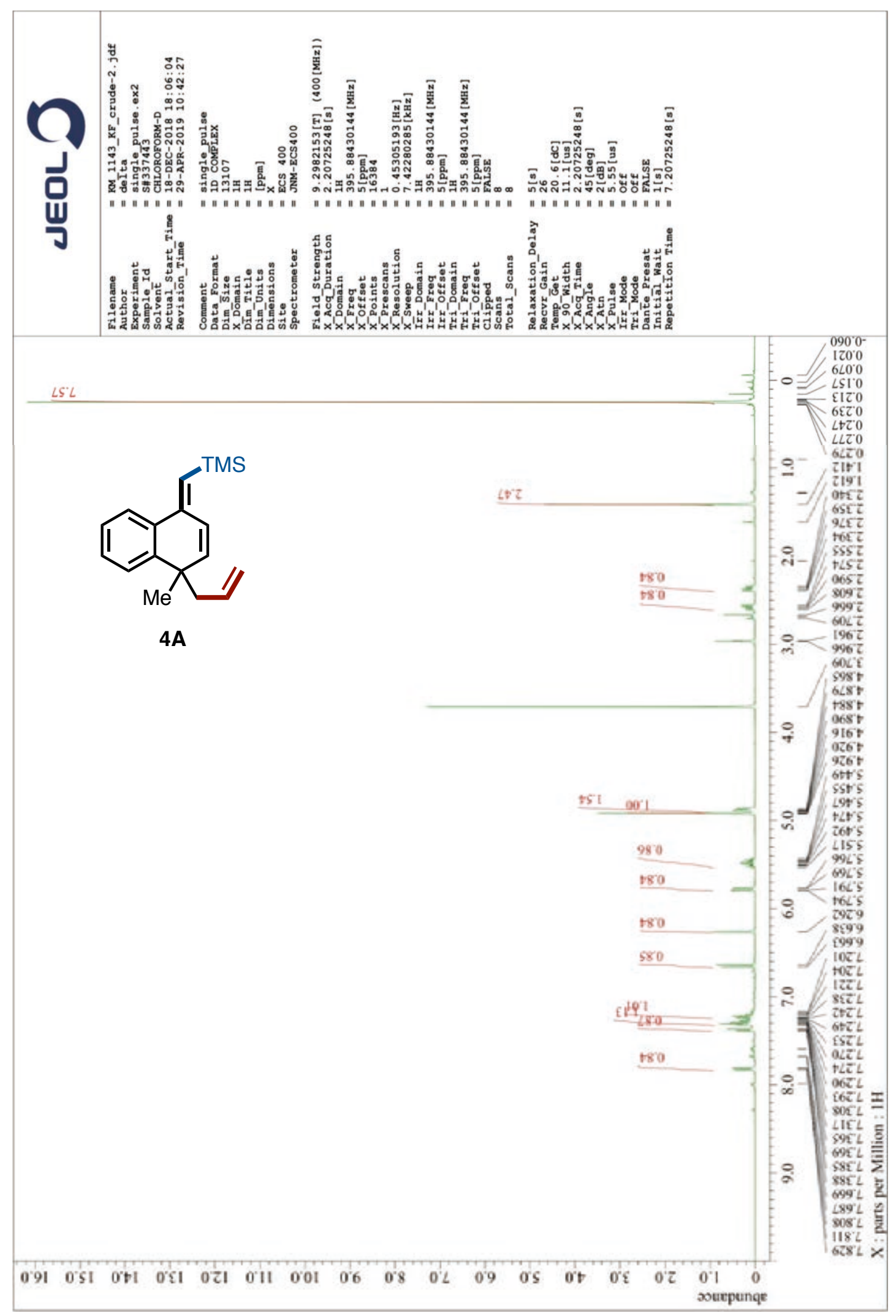


${ }^{1} \mathrm{H}$ NMR of 4B $\left(400 \mathrm{MHz}, \mathrm{CDCl}_{3}\right)$

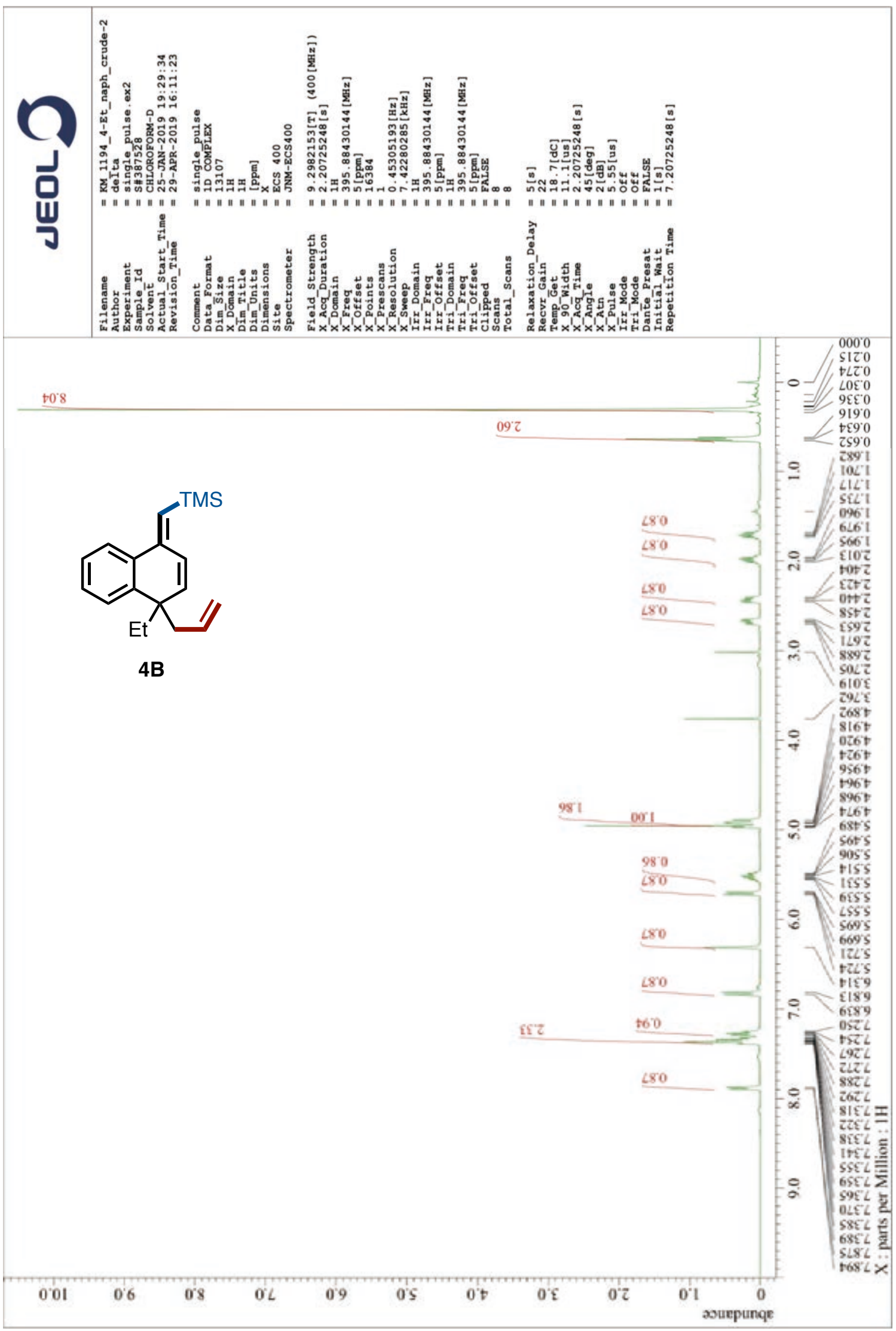


${ }^{1} \mathrm{H}$ NMR of 4C $\left(400 \mathrm{MHz}, \mathrm{CDCl}_{3}\right)$

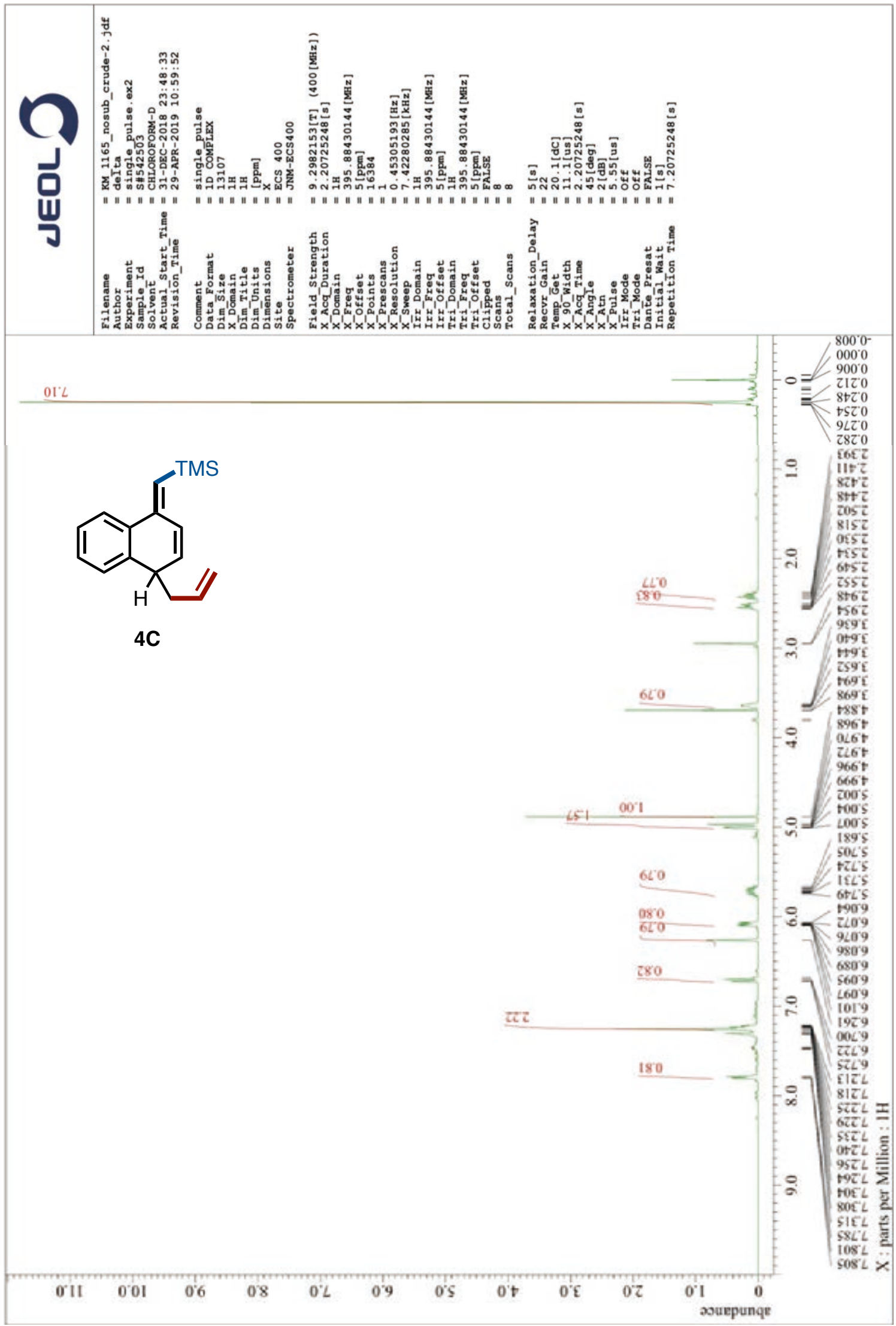


${ }^{1} \mathrm{H}$ NMR of $4 D\left(400 \mathrm{MHz}, \mathrm{CDCl}_{3}\right)$

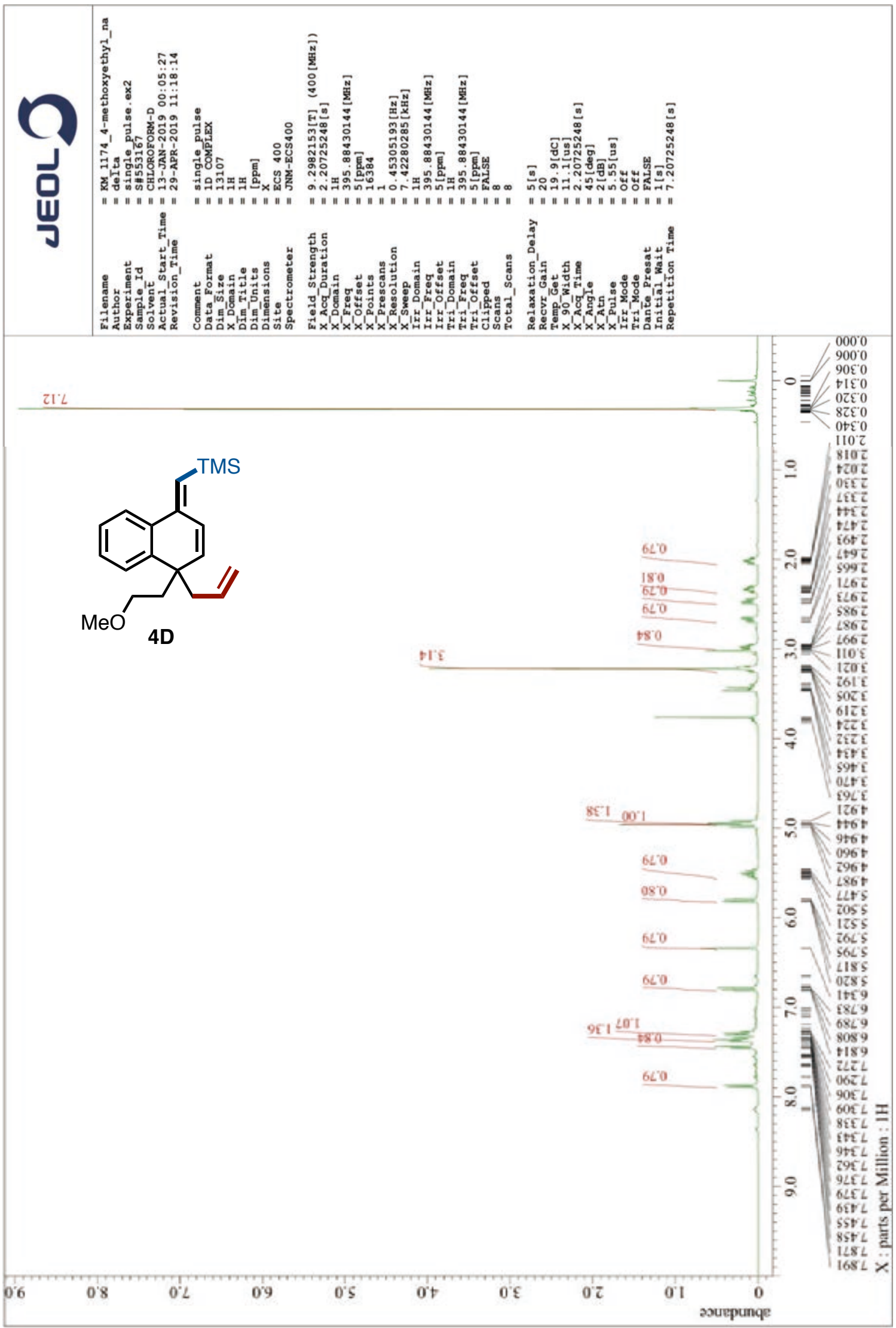


${ }^{1} \mathrm{H}$ NMR of $4 \mathbf{E}\left(400 \mathrm{MHz}, \mathrm{CDCl}_{3}\right)$

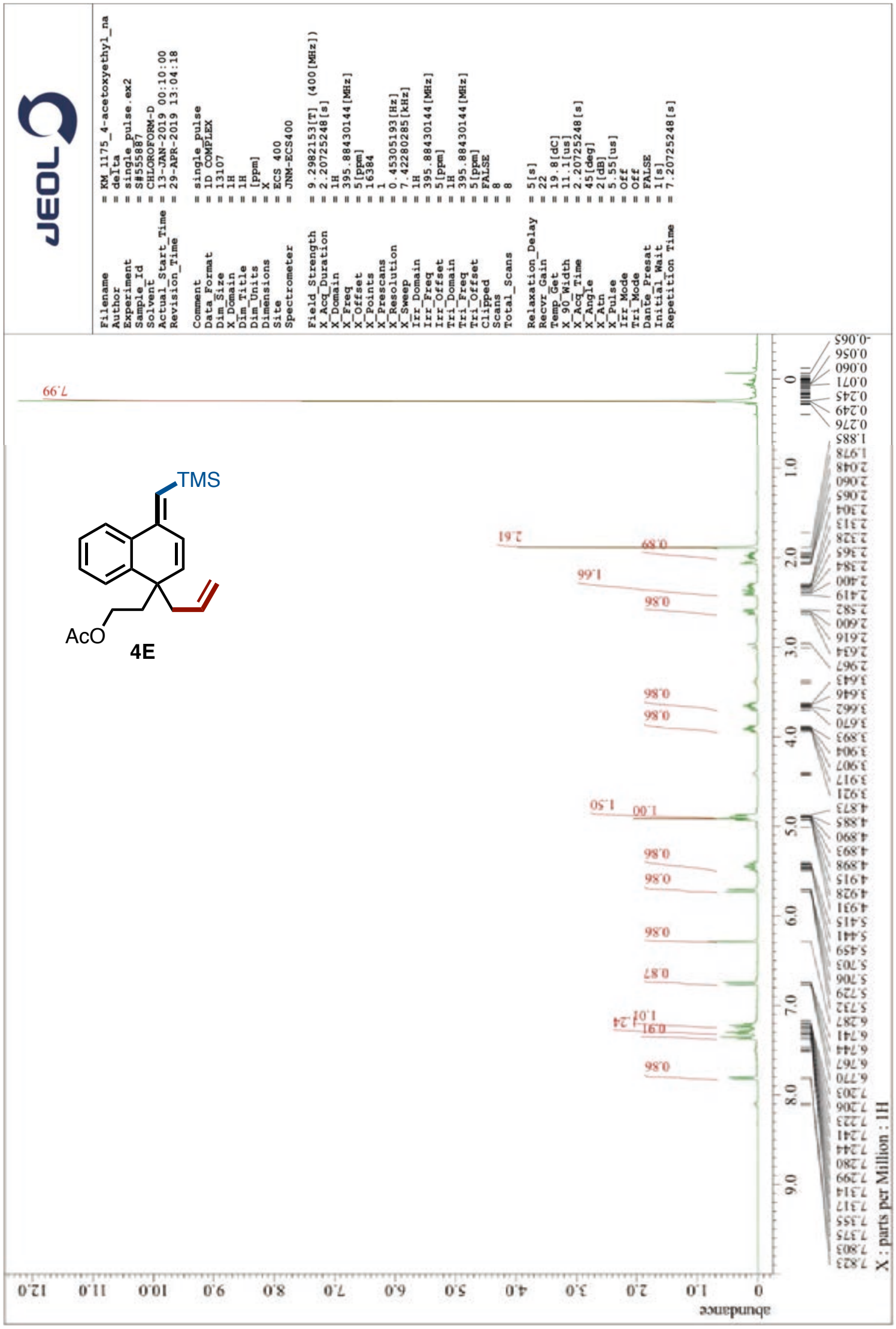


${ }^{1} \mathrm{H}$ NMR of $4 \mathbf{F}\left(400 \mathrm{MHz}, \mathrm{CDCl}_{3}\right)$

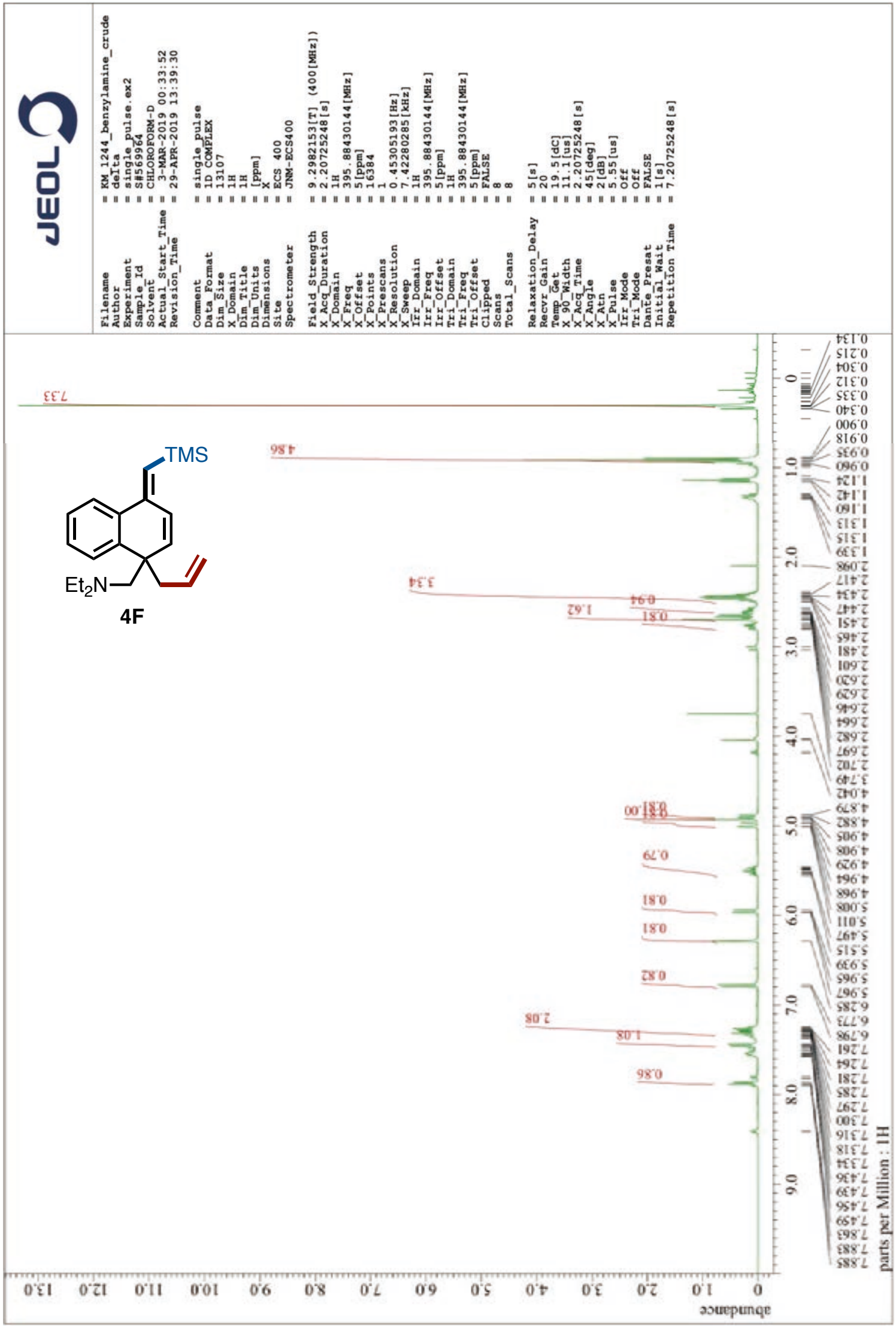


${ }^{1} \mathrm{H} \mathrm{NMR}$ of $\mathbf{4 G}\left(400 \mathrm{MHz}, \mathrm{CDCl}_{3}\right)$

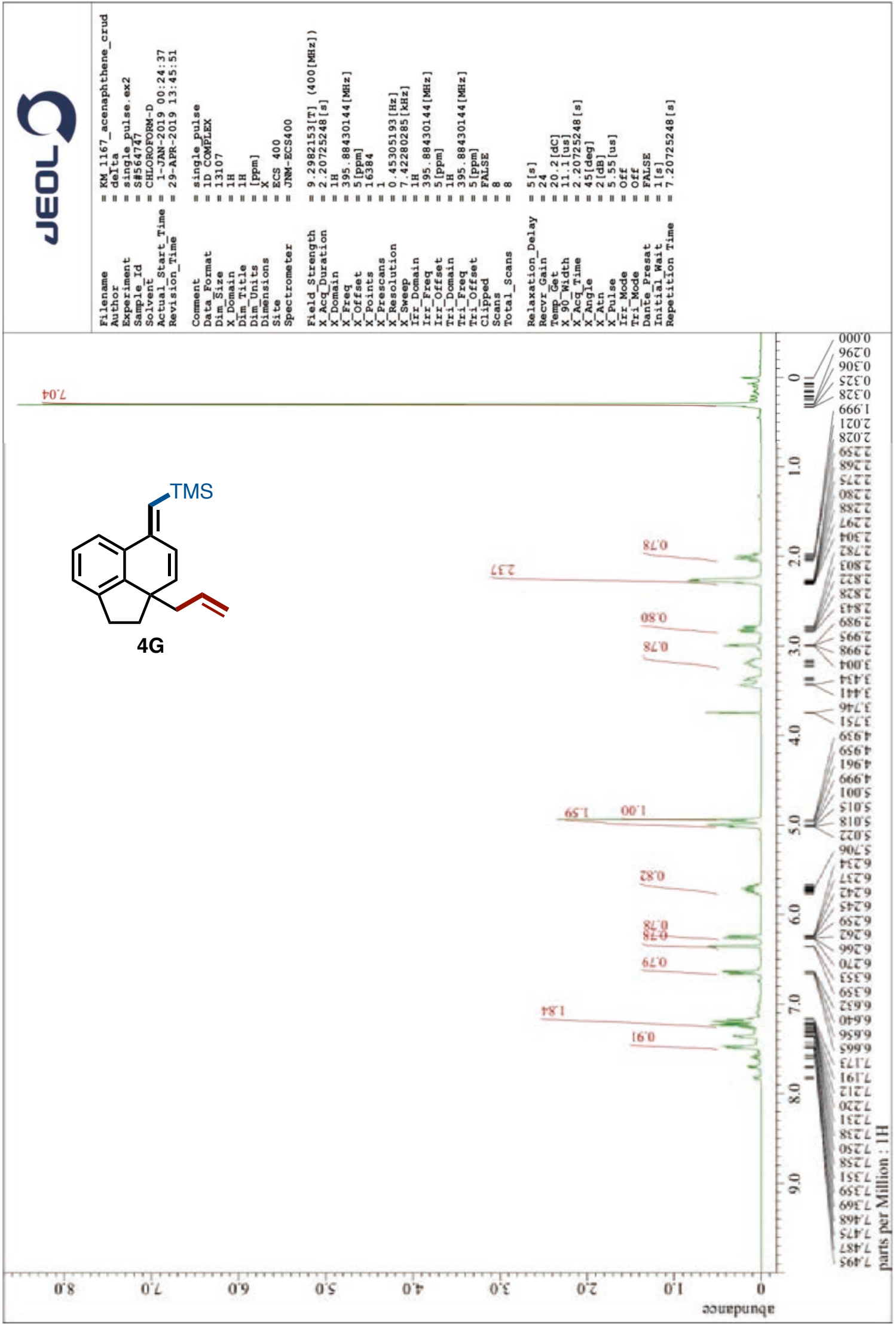


${ }^{1} \mathrm{H} \mathrm{NMR}$ of $\mathbf{4 H}\left(400 \mathrm{MHz}, \mathrm{CDCl}_{3}\right)$

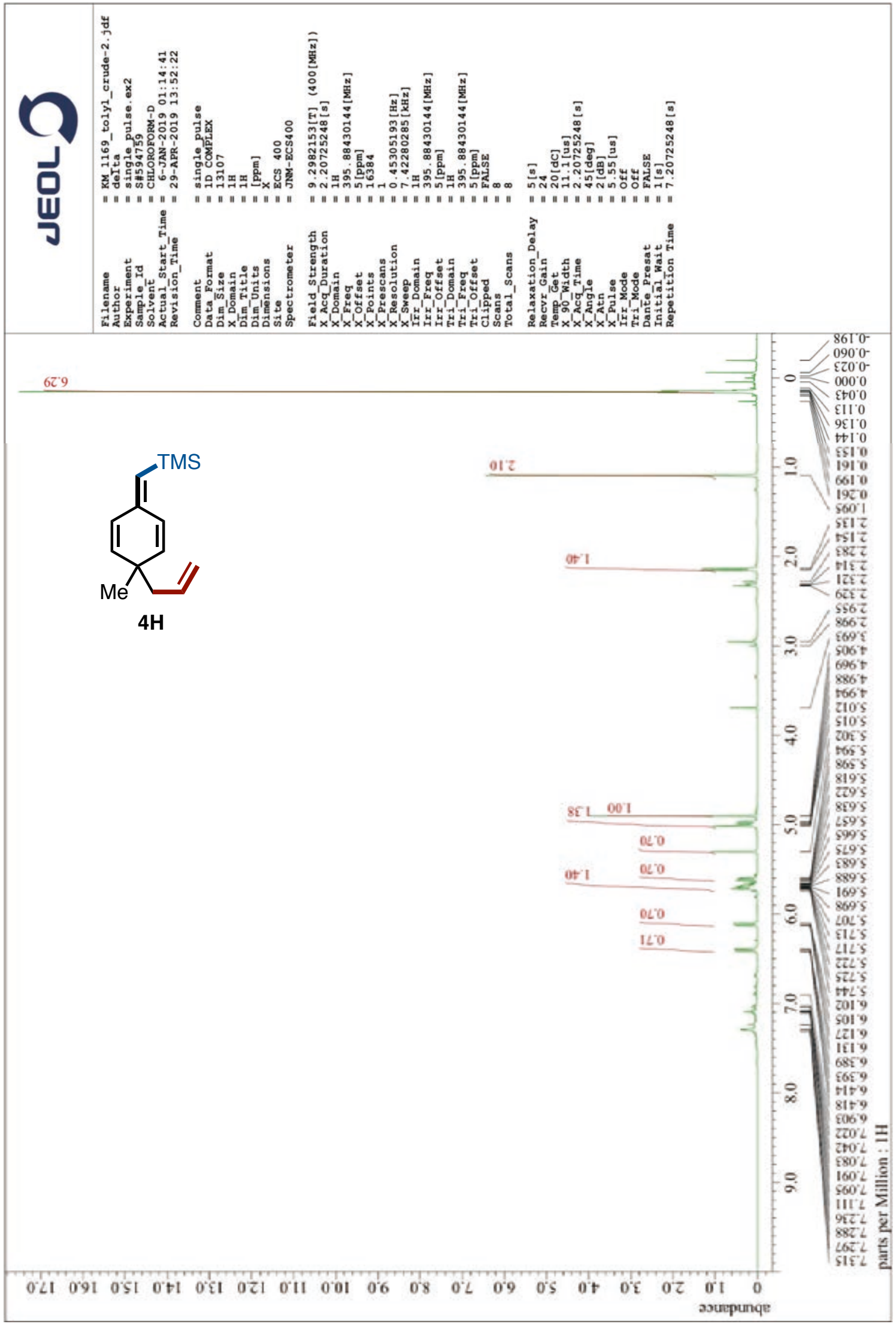


${ }^{1} \mathrm{H}$ NMR of $4 \mathbf{I}\left(400 \mathrm{MHz}, \mathrm{CDCl}_{3}\right)$

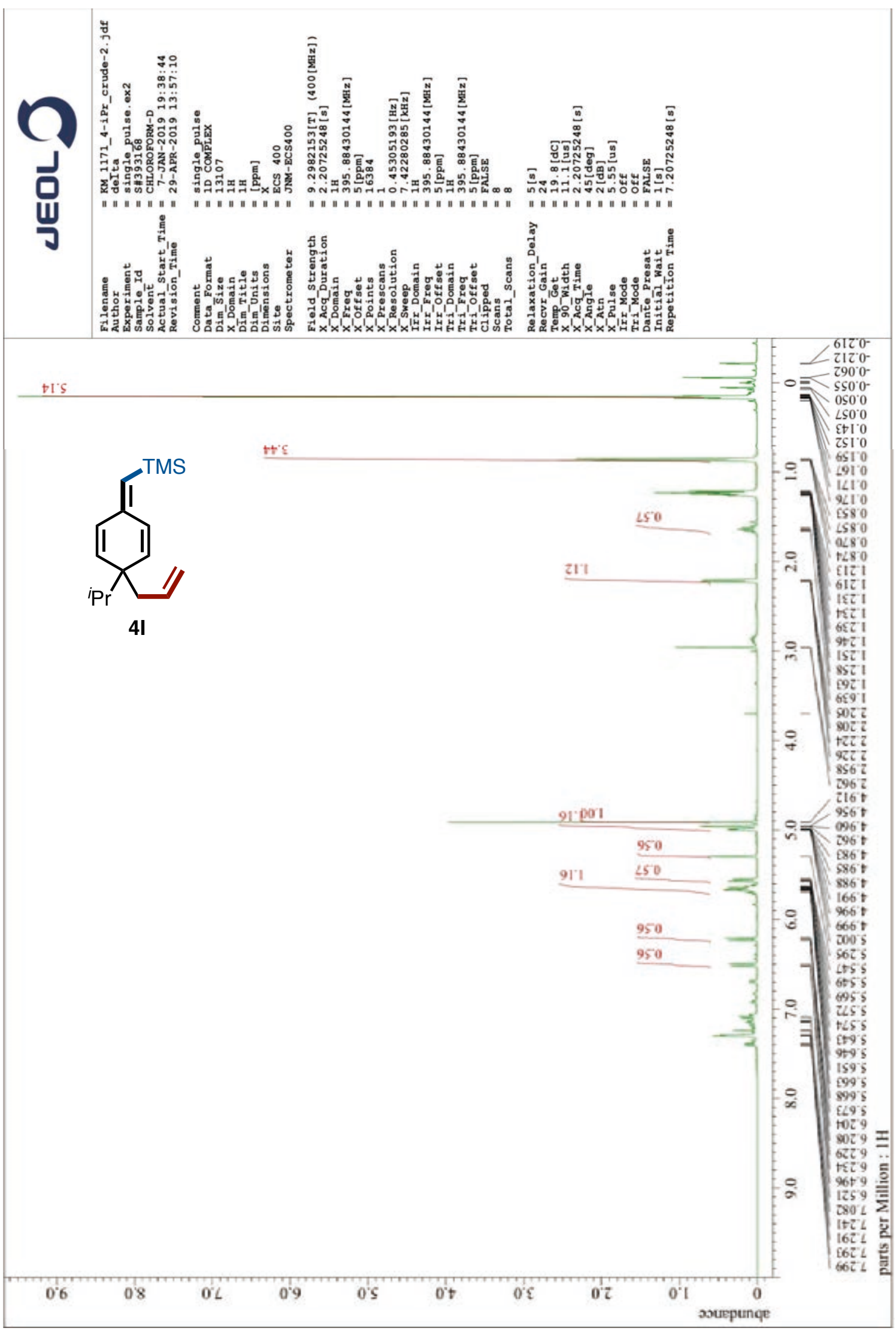


${ }^{1} \mathrm{H}$ NMR of $4 \mathbf{J}\left(400 \mathrm{MHz}, \mathrm{CDCl}_{3}\right)$

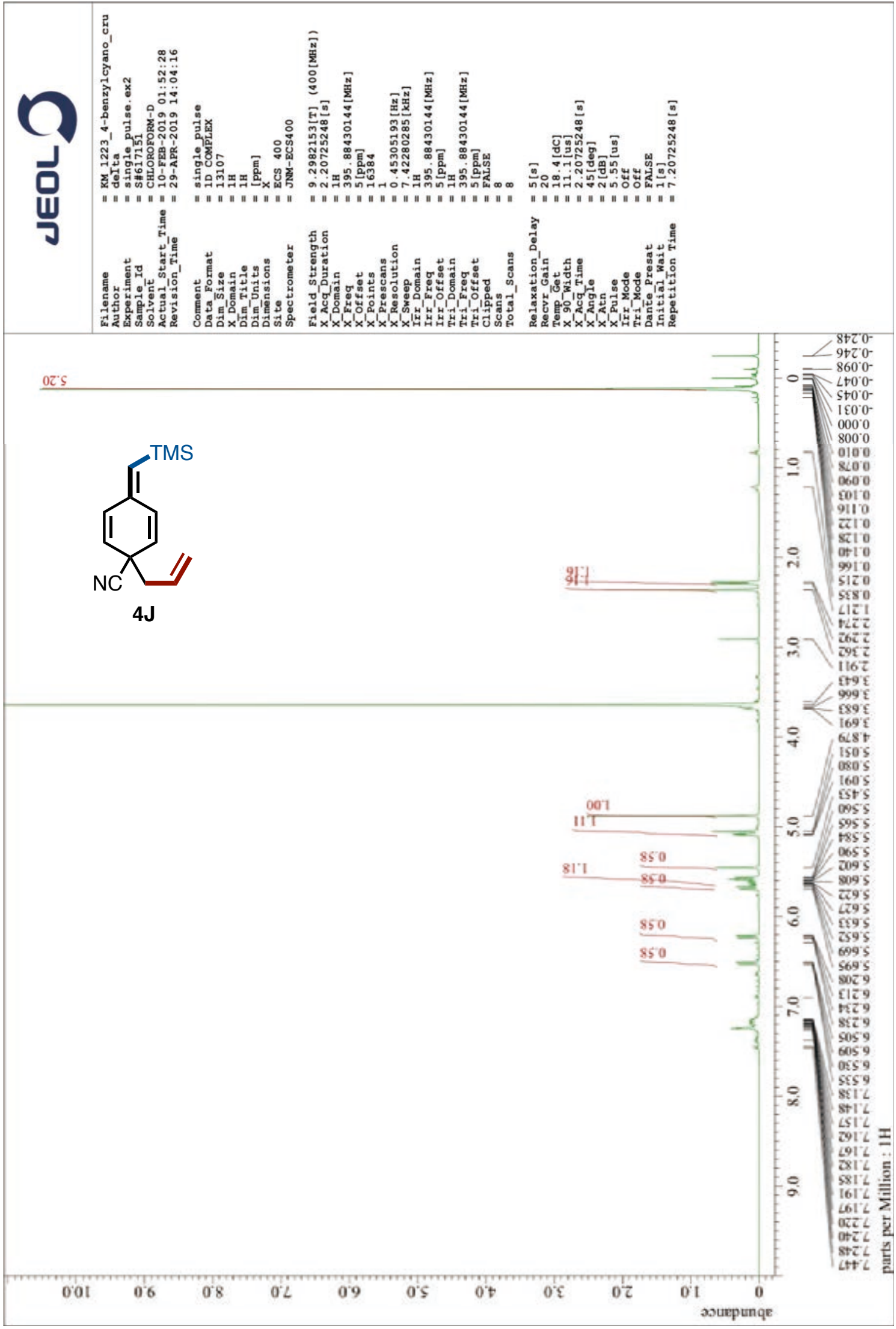


${ }^{1} \mathrm{H}$ NMR of $4 \mathbf{K}\left(400 \mathrm{MHz}, \mathrm{CDCl}_{3}\right)$

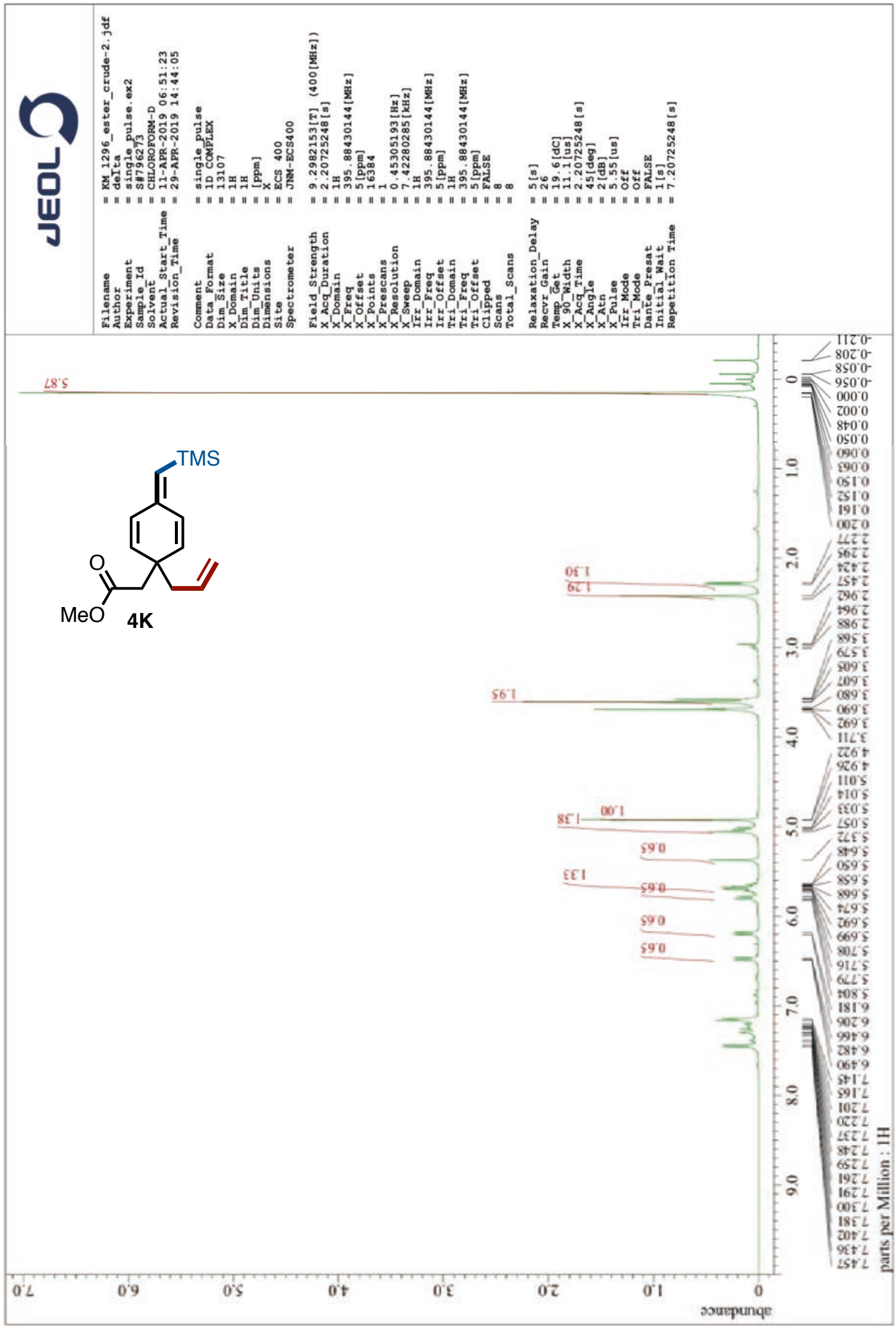


${ }^{1} \mathrm{H} \mathrm{NMR}$ of $\mathbf{4} \mathbf{L}\left(400 \mathrm{MHz}, \mathrm{CDCl}_{3}\right)$

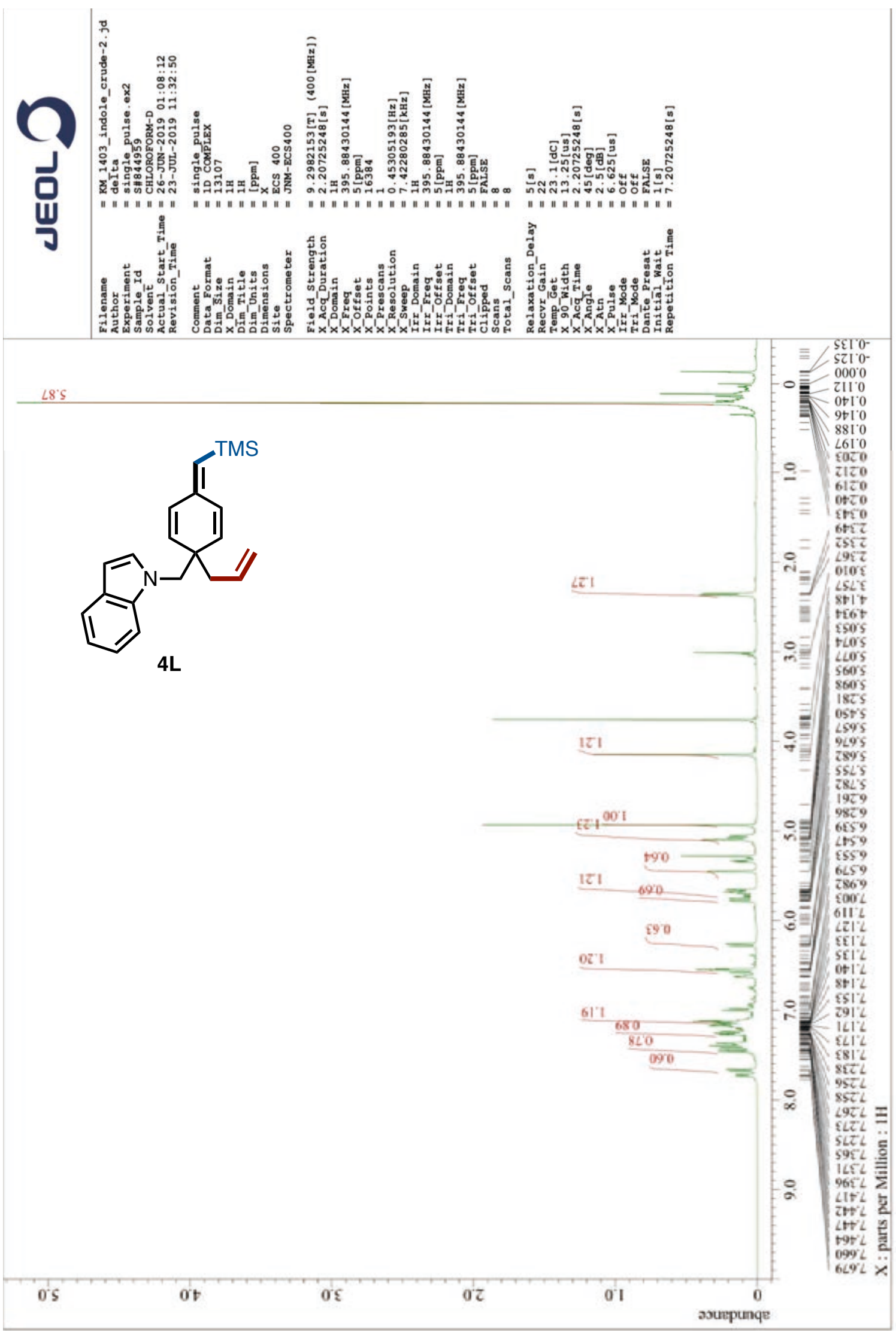


${ }^{1} \mathrm{H}$ NMR of $4 \mathbf{M}\left(400 \mathrm{MHz}, \mathrm{CDCl}_{3}\right)$

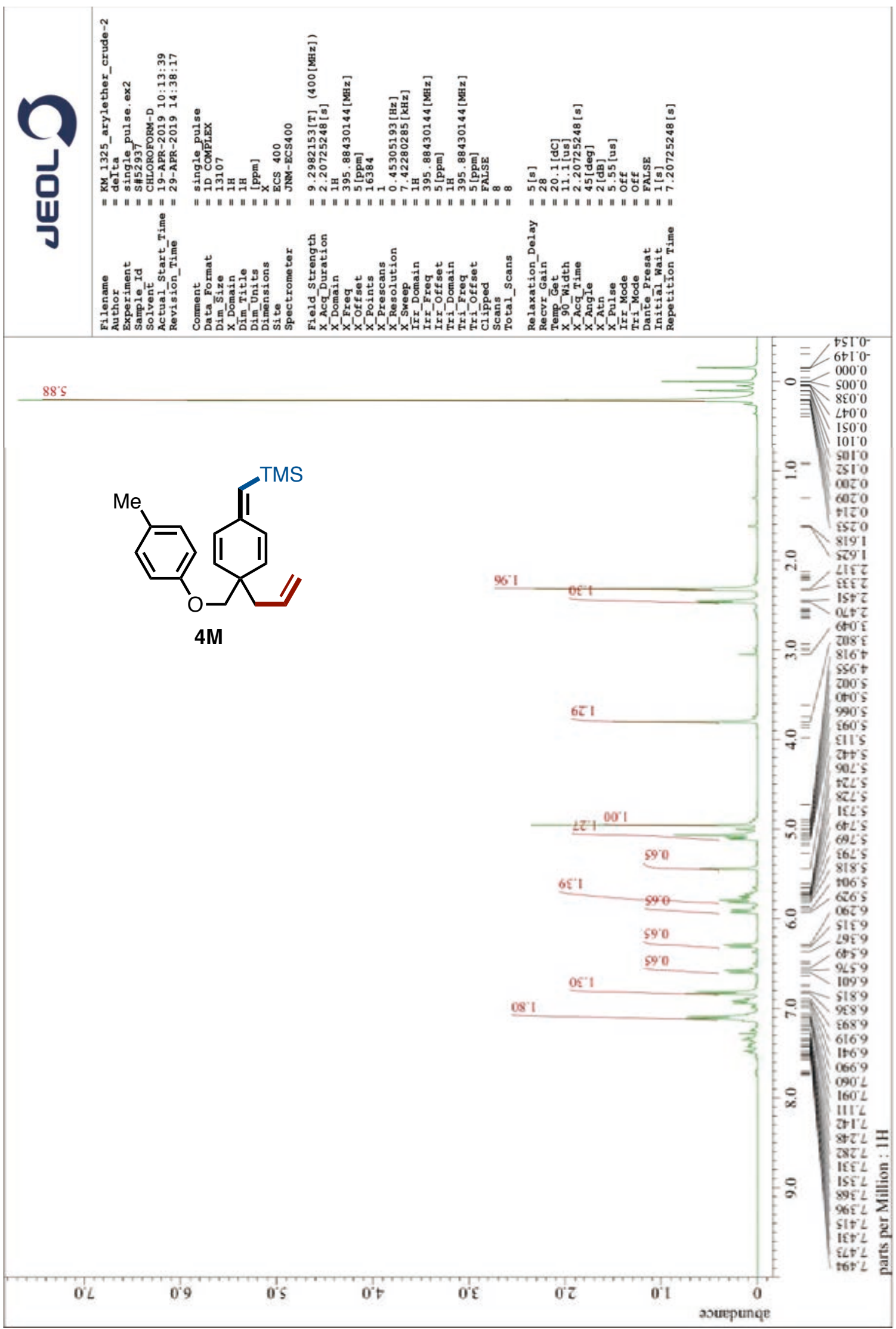


${ }^{1} \mathrm{H}$ NMR of $\mathbf{4 N}\left(400 \mathrm{MHz}, \mathrm{CDCl}_{3}\right)$

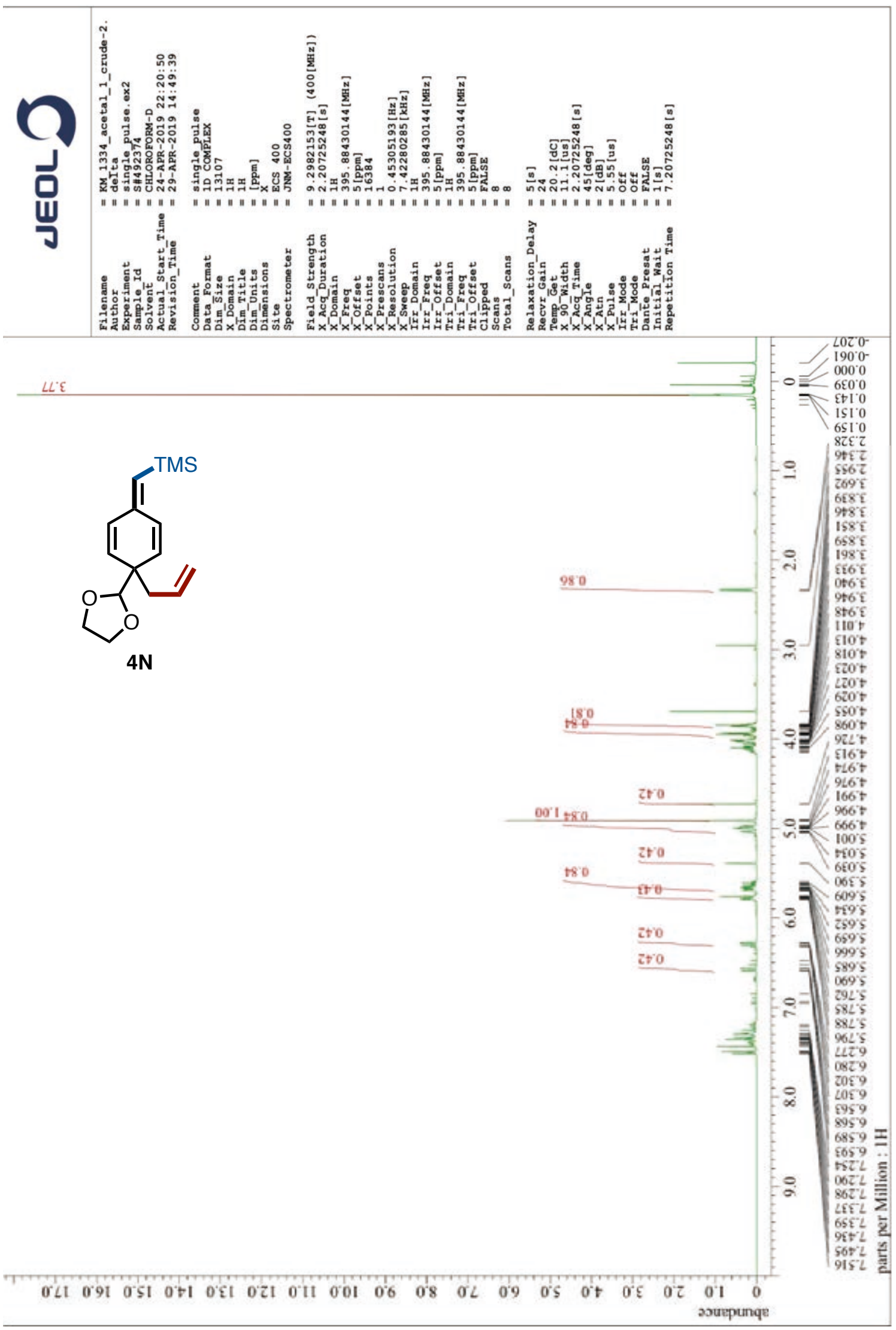


${ }^{1} \mathrm{H}$ NMR of $40\left(400 \mathrm{MHz}, \mathrm{CDCl}_{3}\right)$

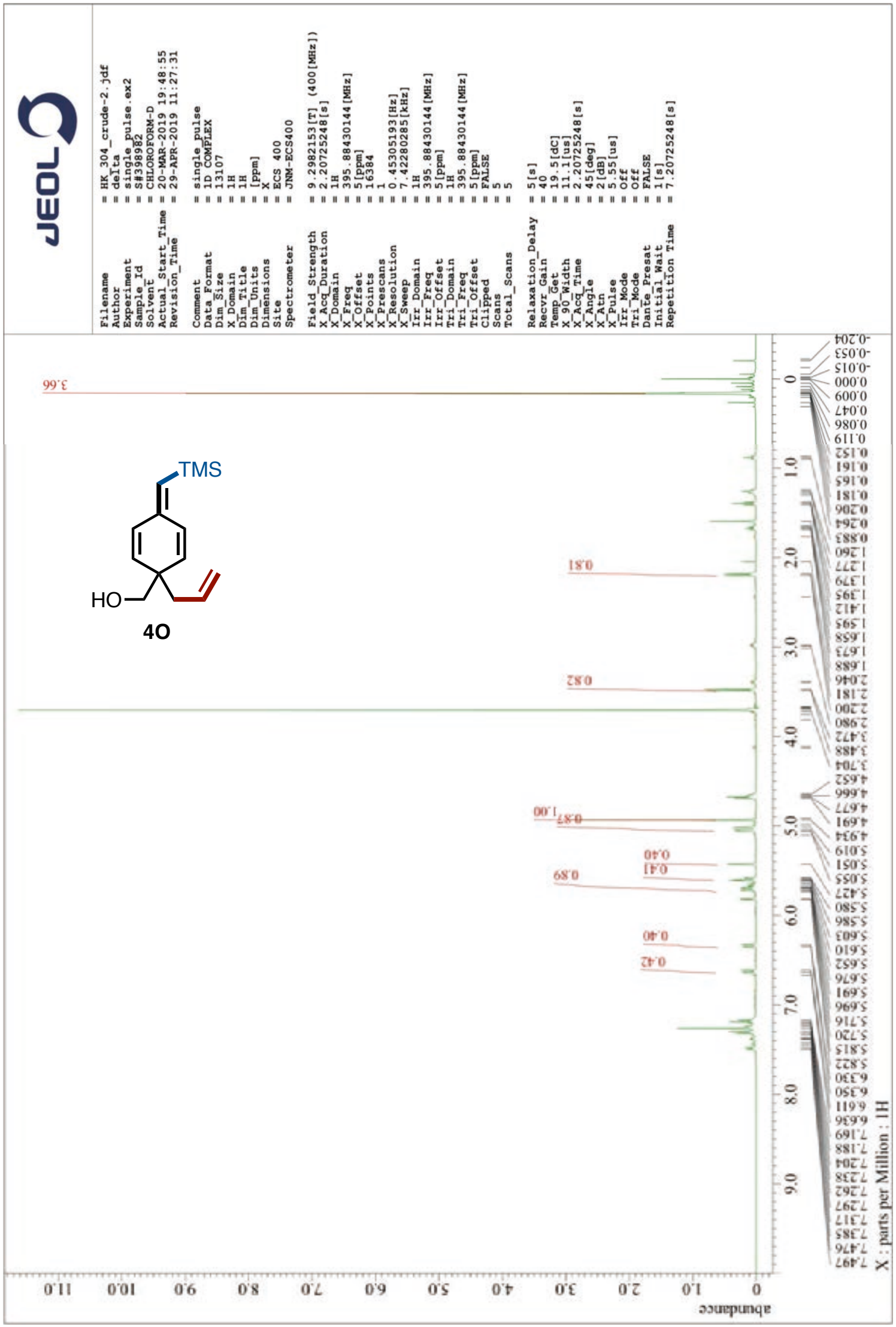


${ }^{1} \mathrm{H}$ NMR of $4 \mathbf{P}\left(400 \mathrm{MHz}, \mathrm{CDCl}_{3}\right)$

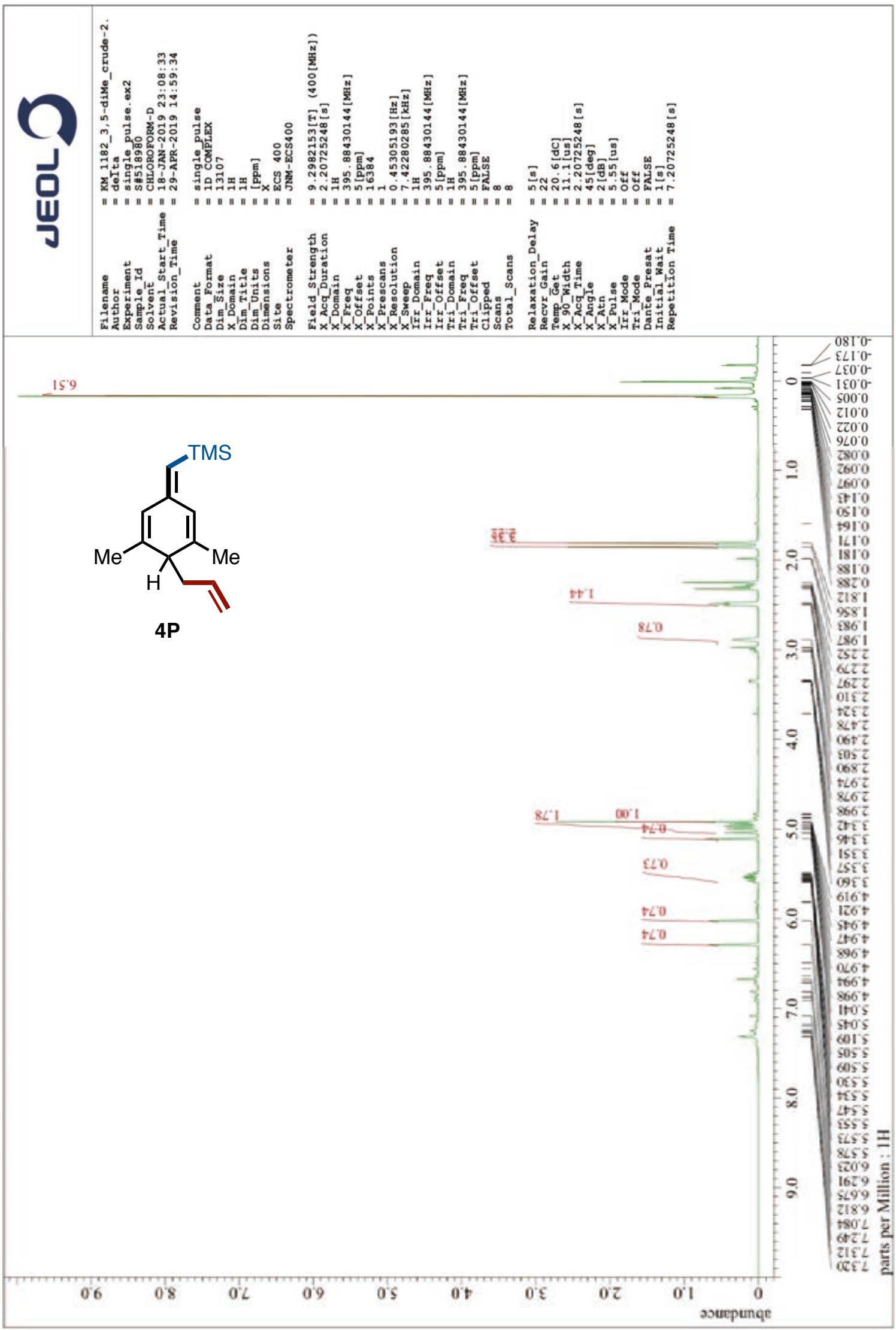


${ }^{1} \mathrm{H}$ NMR of $4 \mathbf{Q}\left(400 \mathrm{MHz}, \mathrm{CDCl}_{3}\right)$

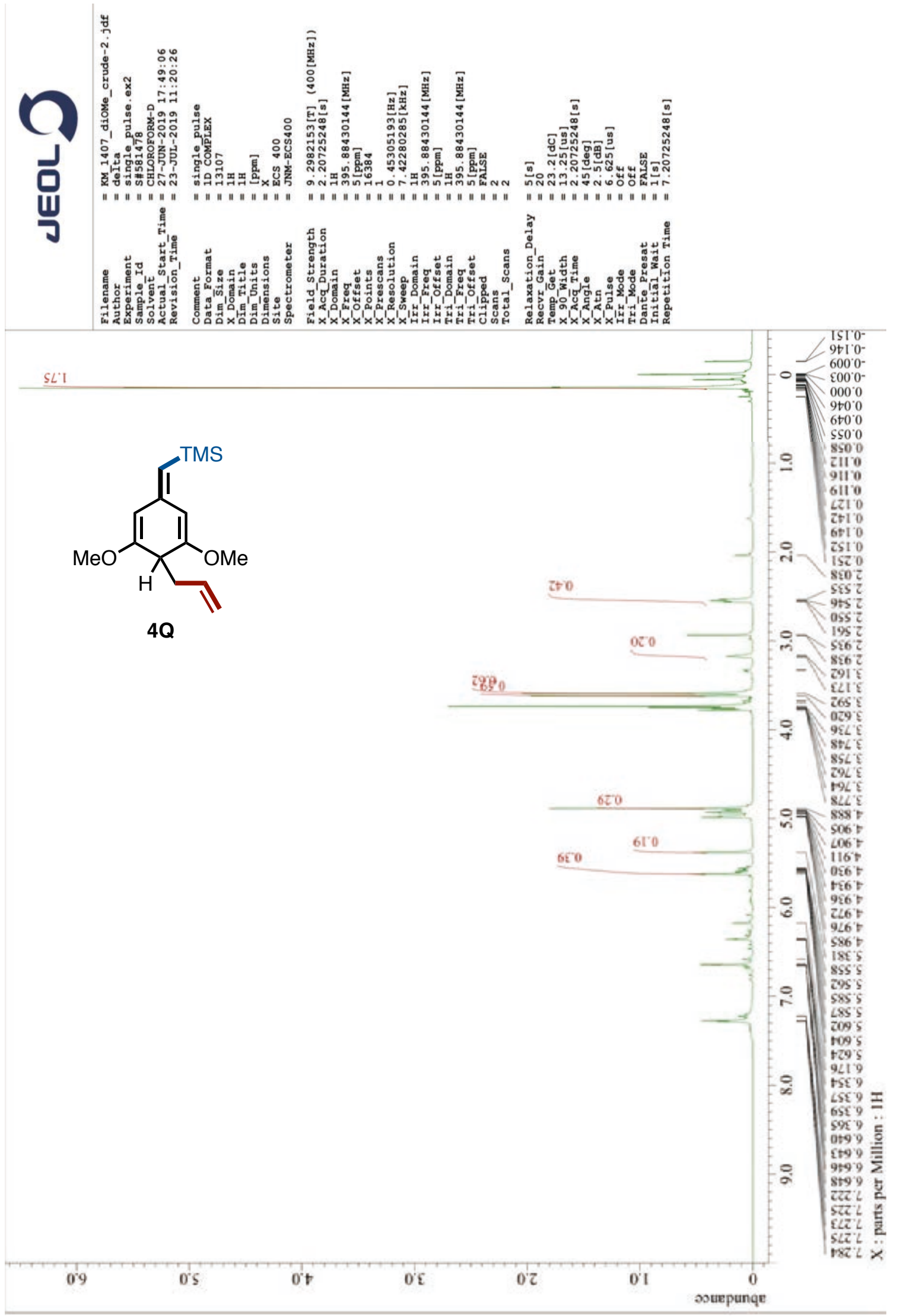


${ }^{1} \mathrm{H}$ NMR of $4 \mathbf{R}\left(400 \mathrm{MHz}, \mathrm{CDCl}_{3}\right)$

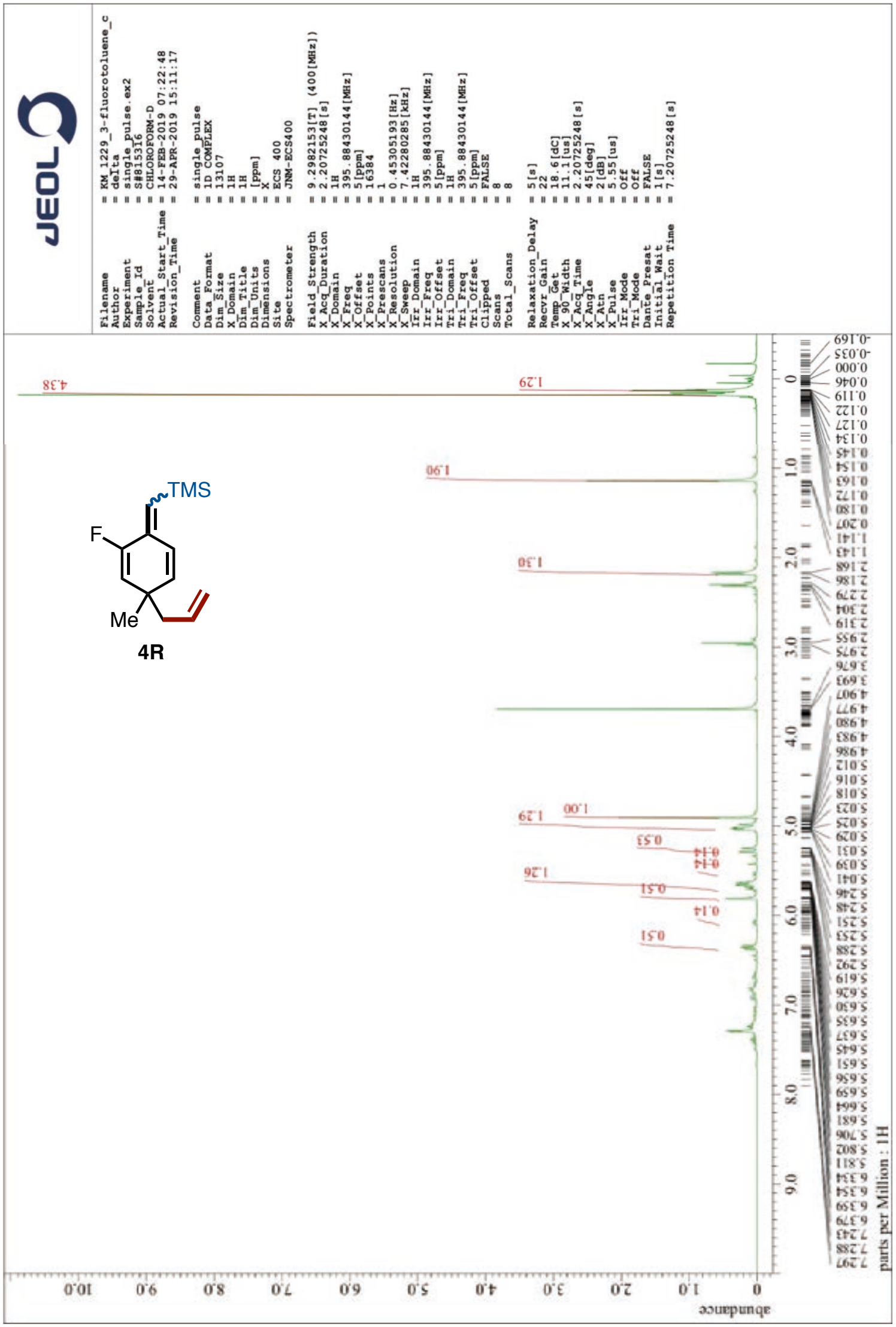


${ }^{1} \mathrm{H}$ NMR of $\mathbf{4 S}\left(400 \mathrm{MHz}, \mathrm{CDCl}_{3}\right)$

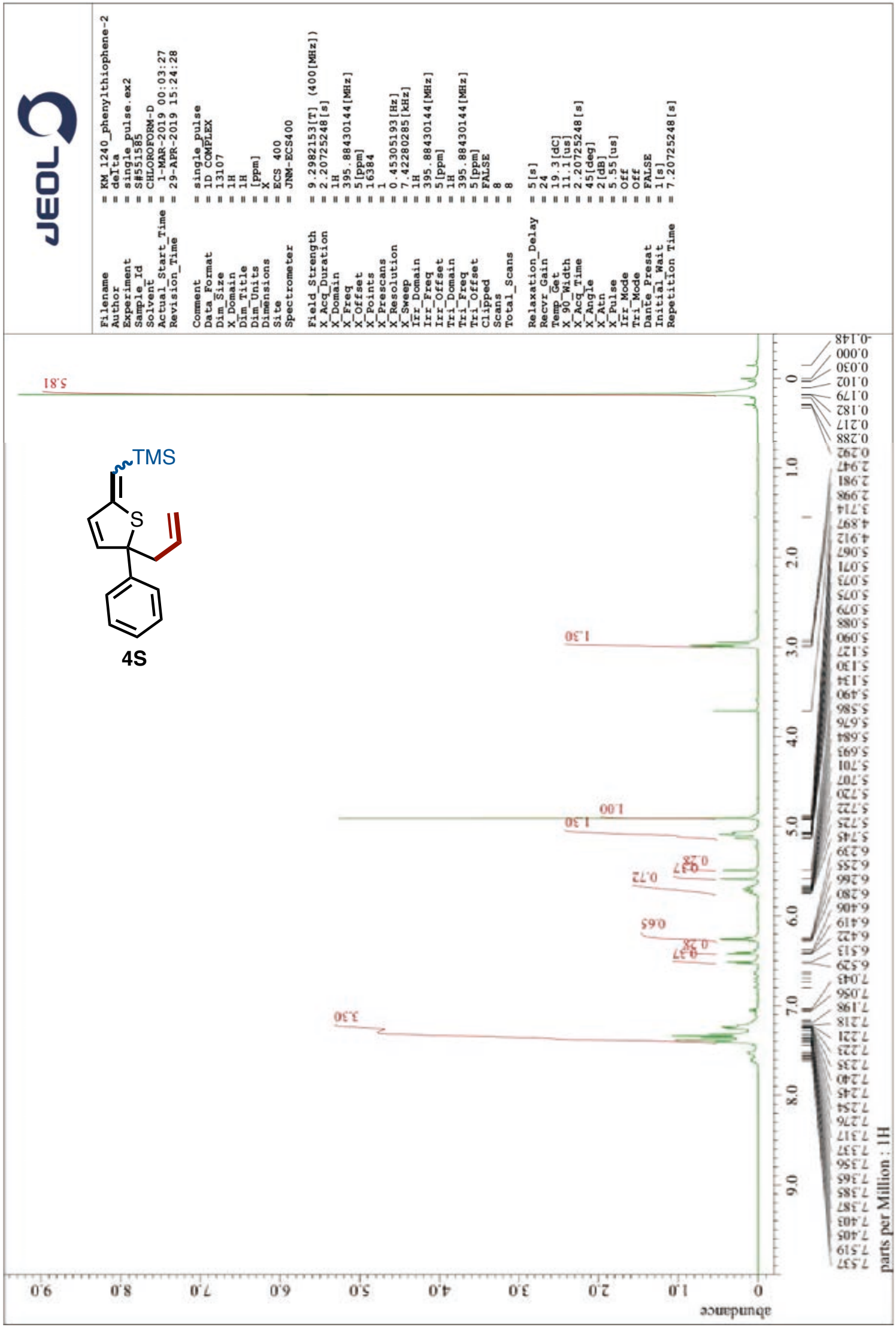


${ }^{1} \mathrm{H}$ NMR of 4 T $\left(400 \mathrm{MHz}, \mathrm{CDCl}_{3}\right)$

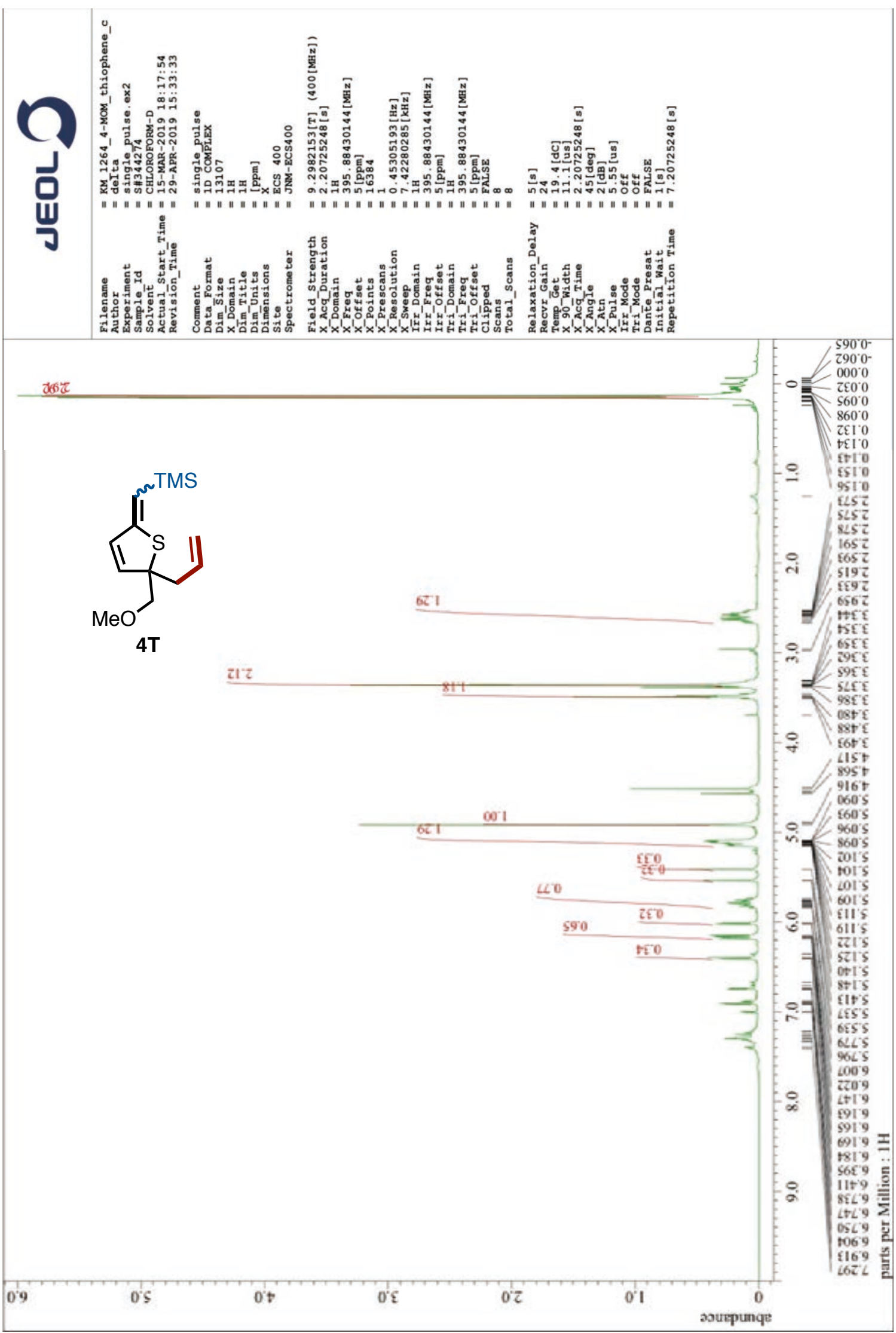


${ }^{1} \mathrm{H} \mathrm{NMR}$ of $\mathbf{4} \mathbf{U}\left(400 \mathrm{MHz}, \mathrm{CDCl}_{3}\right)$

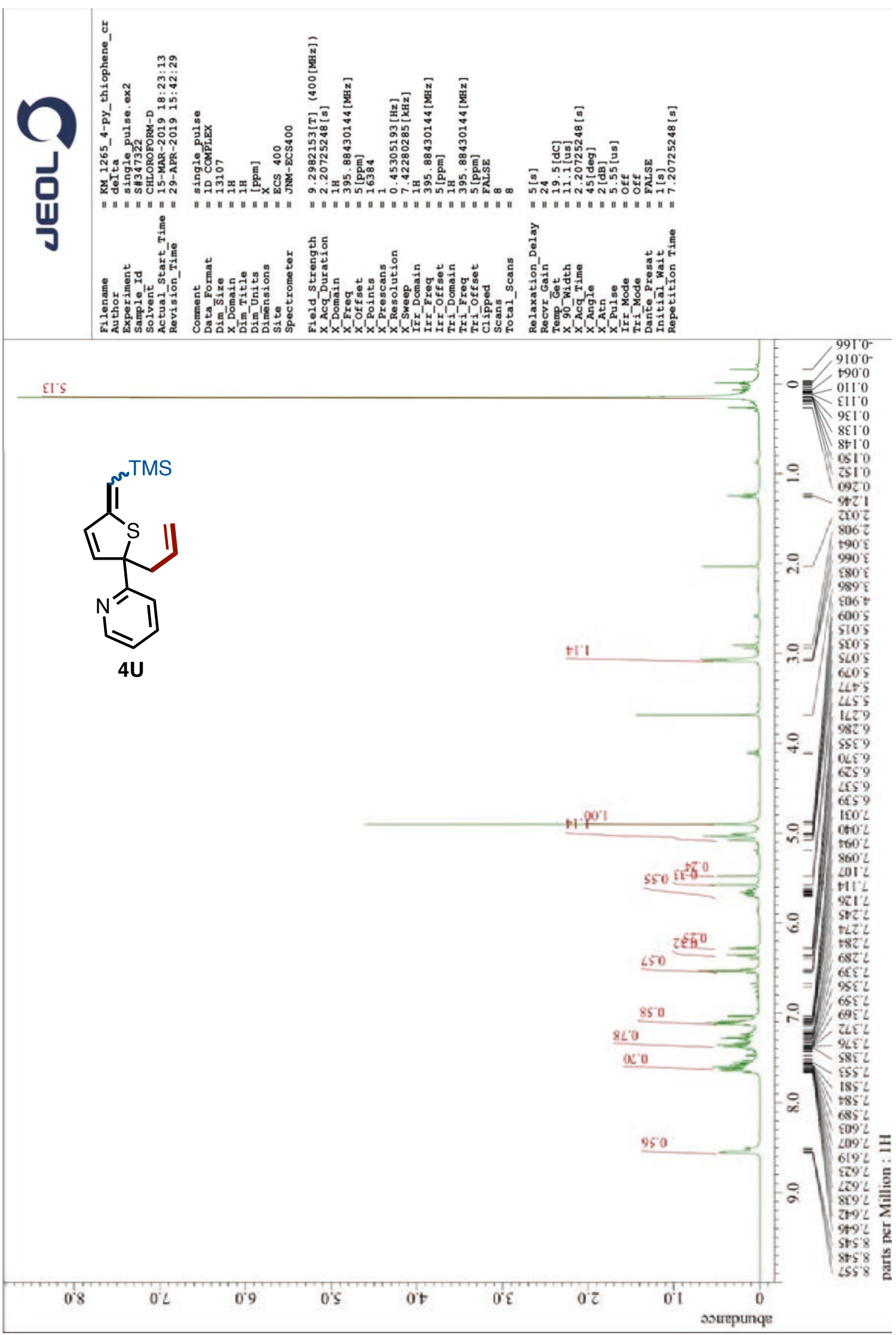


${ }^{1} \mathrm{H} \mathrm{NMR}$ of $4 \mathbf{V}\left(400 \mathrm{MHz}, \mathrm{CDCl}_{3}\right)$

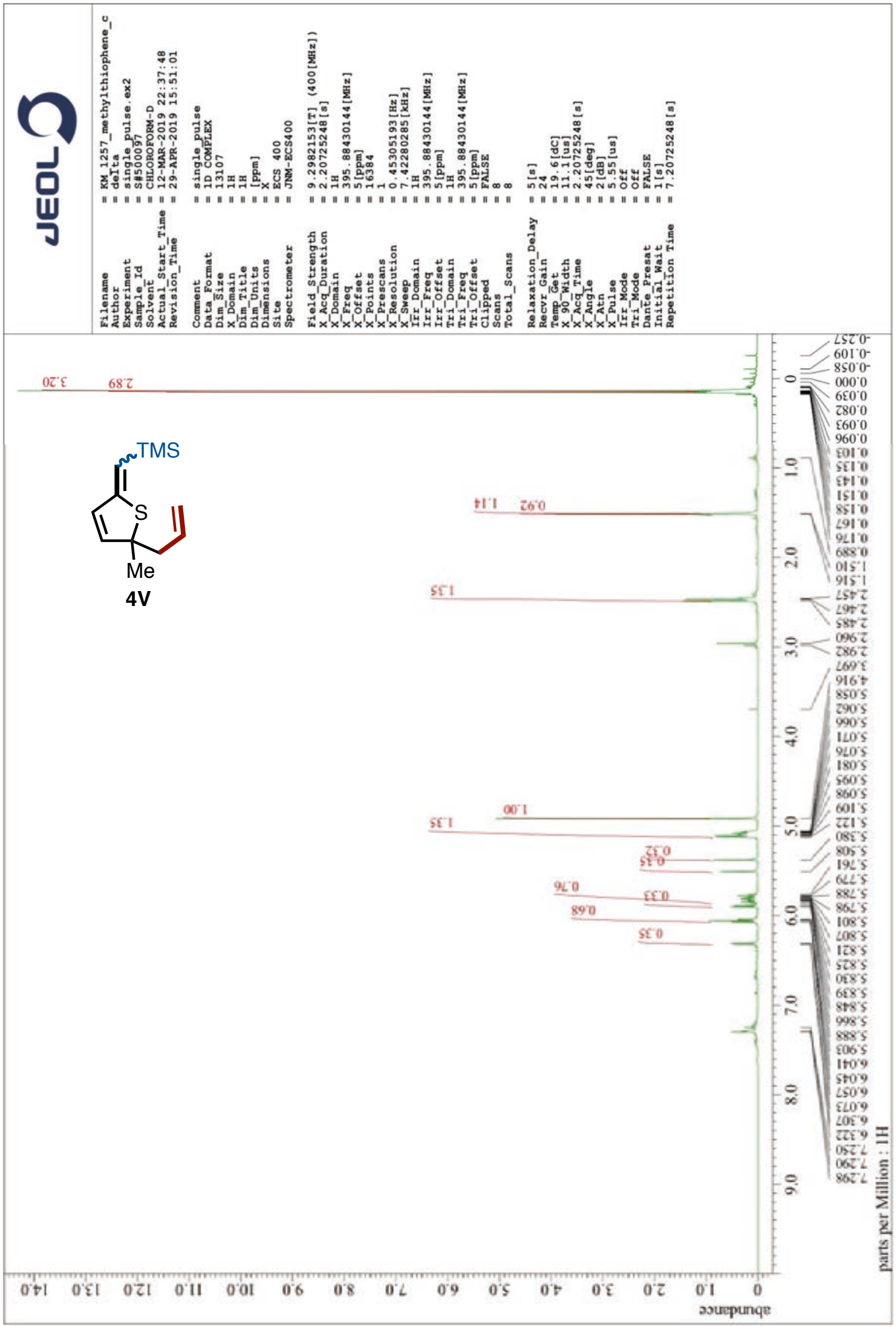


${ }^{1} \mathrm{H}$ NMR of $\mathbf{4 W}\left(400 \mathrm{MHz}, \mathrm{CDCl}_{3}\right)$

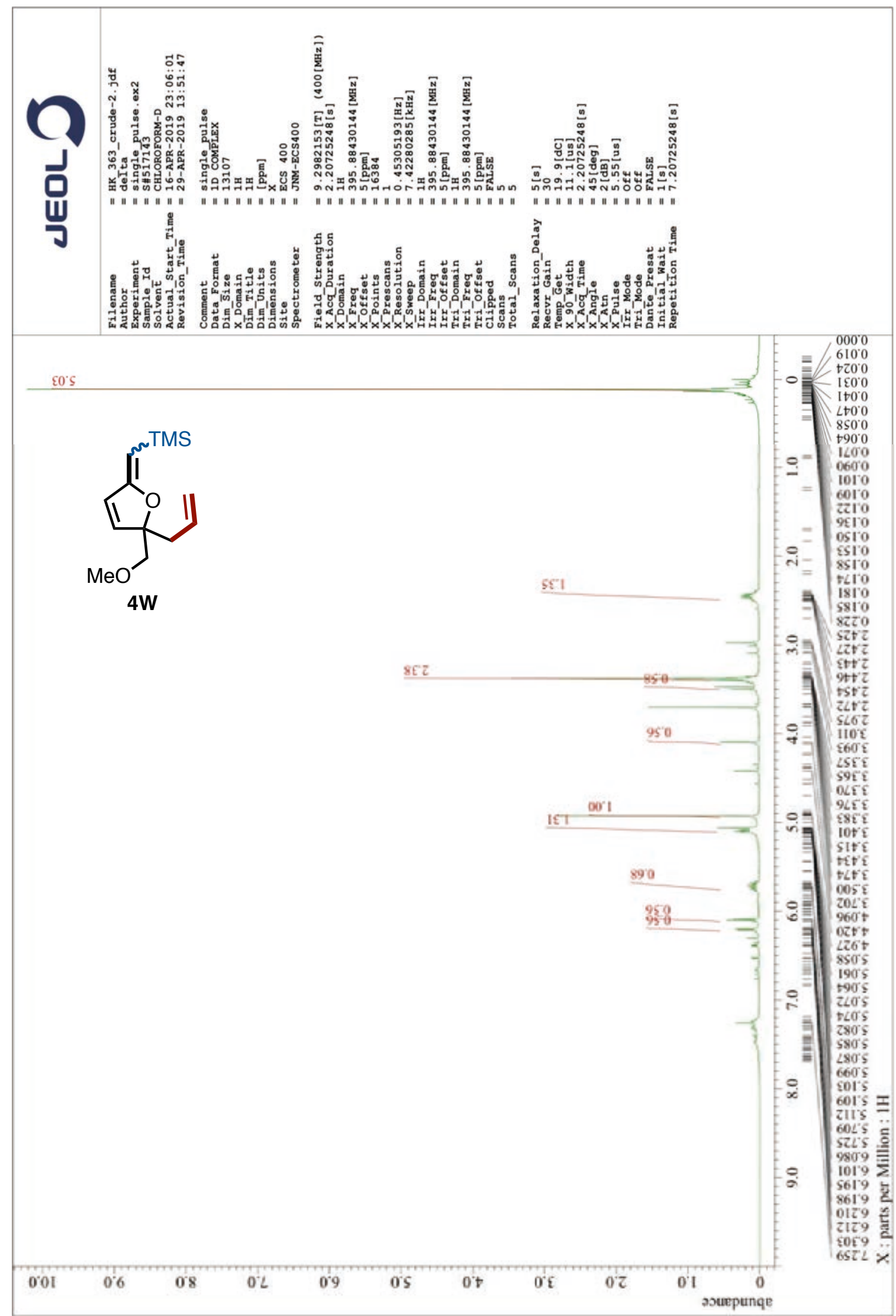


${ }^{1} \mathrm{H}$ NMR of $4 \mathbf{X}\left(400 \mathrm{MHz}, \mathrm{CDCl}_{3}\right)$

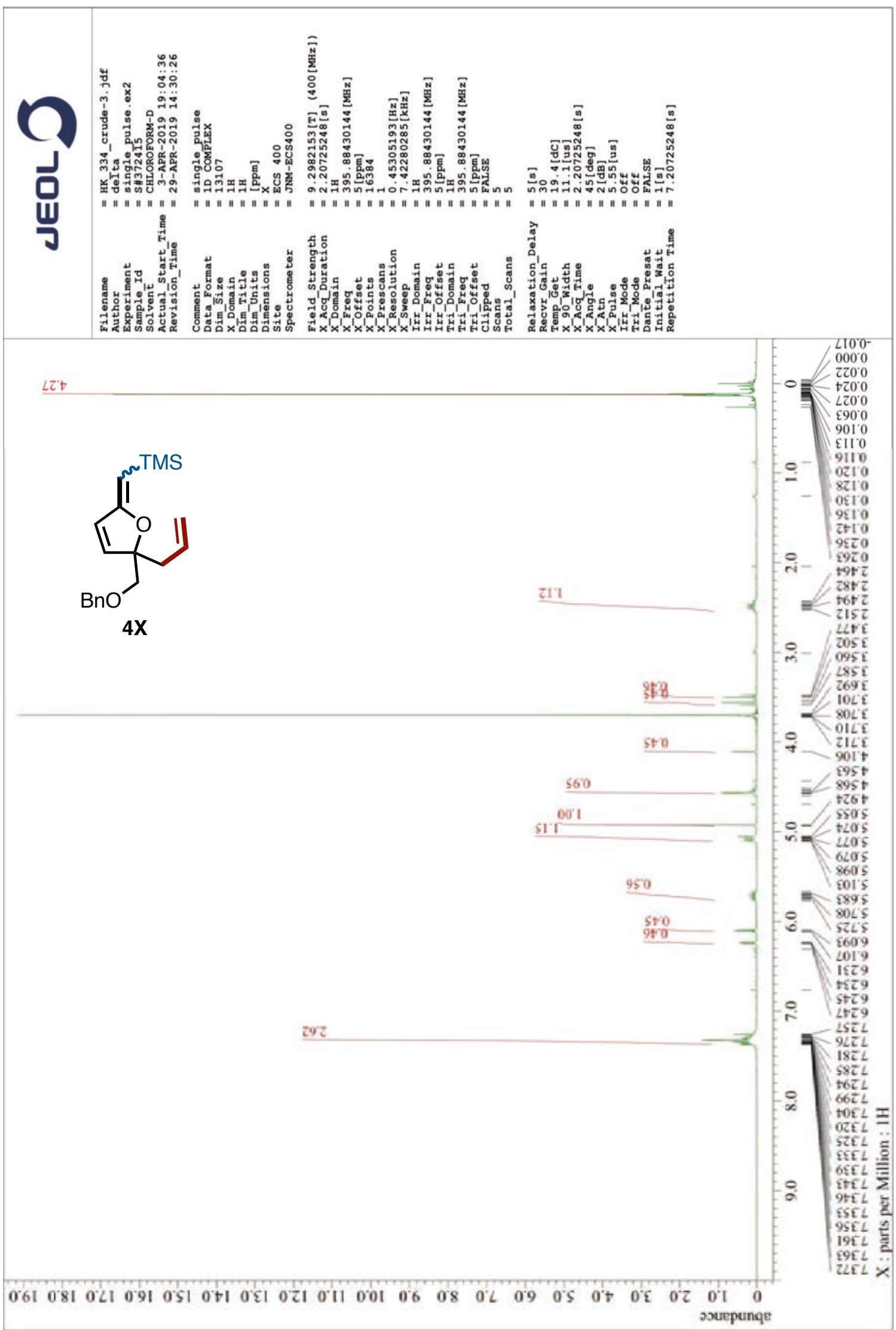


${ }^{1} \mathrm{H}$ NMR of $8\left(400 \mathrm{MHz}, \mathrm{CDCl}_{3}\right)$

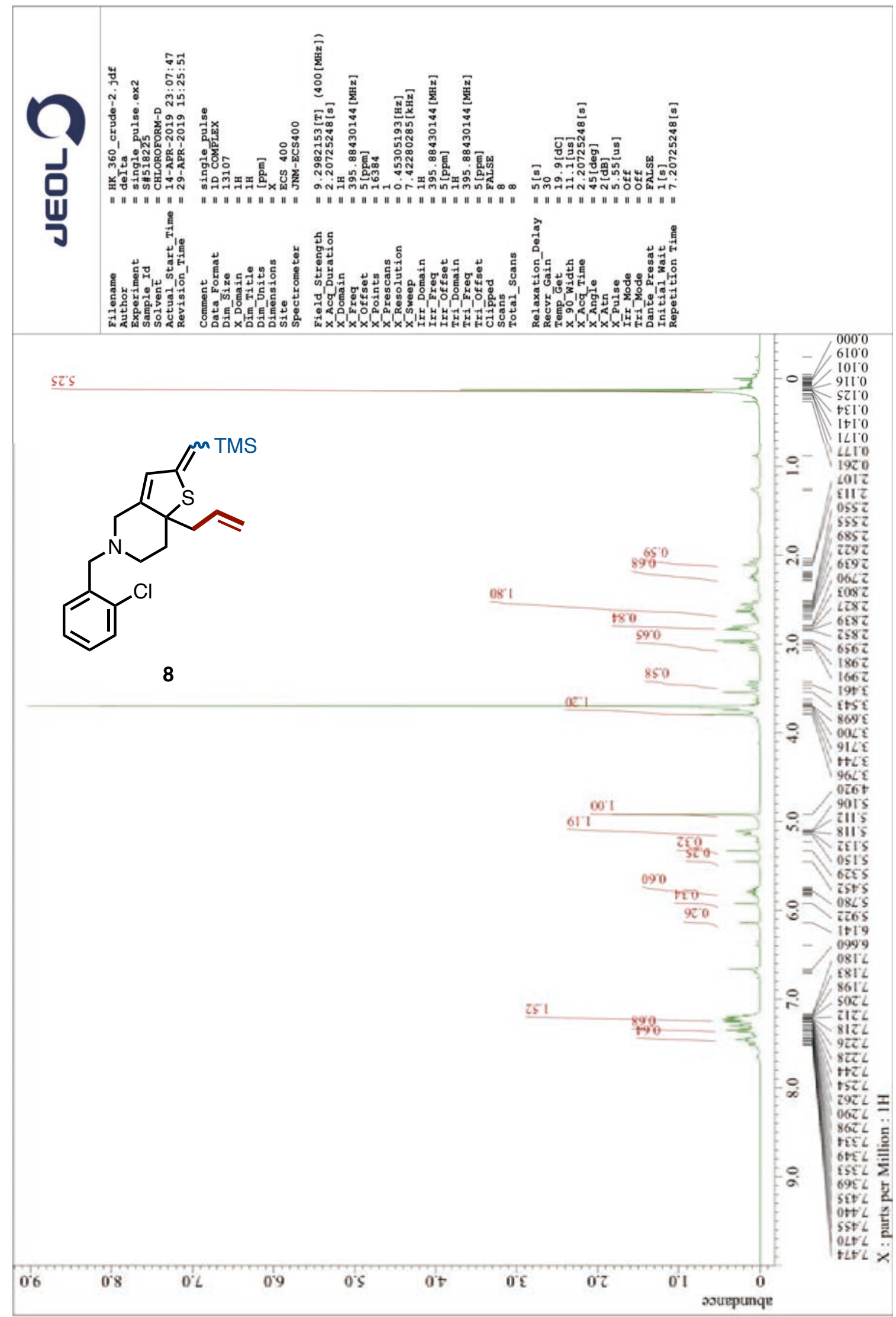

